\title{
ELECTRICALLY RESPONSIVE FLUOROPOLYMER SURFACES AND APPLICATIONS
}




\section{ELECTRICALLY RESPONSIVE FLUOROPOLYMER SURFACES AND APPLICATIONS}

$\mathrm{Hao} \mathrm{Wu}$ 



\title{
ELECTRICALLY RESPONSIVE FLUOROPOLYMER SURFACES AND APPLICATIONS
}

\author{
DISSERTATION
}

to obtain

the degree of doctor at the Universiteit Twente,

on the authority of the rector magnificus,

Prof.dr. T.T.M. Palstra,

on account of the decision of the graduation committee,

to be publicly defended

on Wednesday $4^{\text {th }}$ of March 2020 at 16.45

by

Hao Wu

Born on the $7^{\text {th }}$ of April 1988

in Xinxiang, China 
This dissertation has been approved by:

Prof. dr. F.G. Mugele

Promotor

Prof. dr. G. Zhou

Co-Promotor

Title: Electrically responsive fluoropolymer surfaces and applications

Author: Hao Wu

Cover design: $\mathrm{Hao} \mathrm{Wu}$

Printed by: Ipskamp Printing, Enschede

ISBN: 978-90-365-4952-3

DOI: $10.3990 / 1.9789036549523$

(C) $2020 \mathrm{Hao} \mathrm{Wu}$, The Netherlands. All rights reserved. No parts of this thesis may be reproduced, stored in a retrieval system or transmitted in any form or by any means without permission of the author. Alle rechten voorbehouden. Niets uit deze uitgave mag worden vermenigvuldigd, in enige vorm of op enige wijze, zonder voorafgaande schriftelijke toestemming van de auteur. 


\section{Graduation Committee:}

\section{Chairman}

Prof. dr. J.L. Herek

University of Twente

\section{Promotor}

Prof. dr. F.G. Mugele

University of Twente

\section{Co-Promotor}

Prof. dr. G. Zhou

South China Normal University

\section{Committee Members:}

Prof. dr. S. A. L.Weber

Prof. dr. A. Darhuber

Prof. dr. J.C.T. Eijkel

Prof. dr. IR. H.J.W. Zandvliet

Max Planck Institute for Polymer Research Eindhoven University of Technology

Prof. dr. S.J.G. Lemay

University of Twente

University of Twente

University of Twente

The research described in this thesis was conducted at the Physics of Complex Fluids group of the MESA+ Institute for Nanotechnology, University of Twente and Institute of Electronic Paper Displays of South China Academy of Advanced Optoelectronics, South China Norma University.

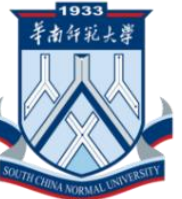



To my beloved family.

献给我最亲爱的家人 



\section{Contents}

\section{Chapter 1}

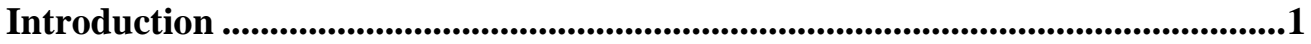

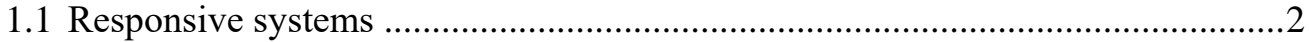

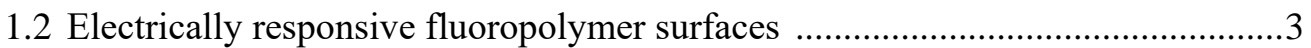

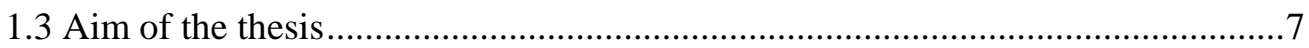

1.4 Thesis outline

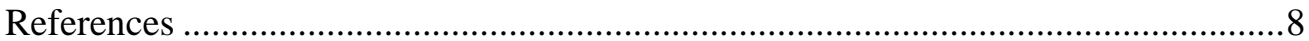

\section{Chapter 2}

Influence of fluoropolymer surface wettability on electrowetting display

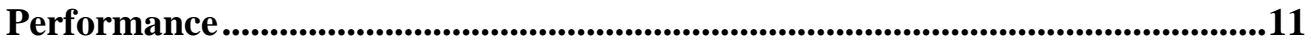

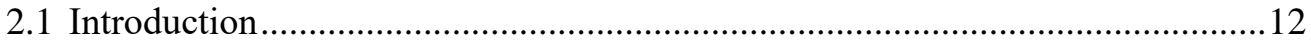

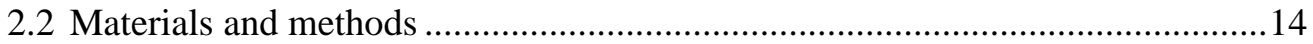

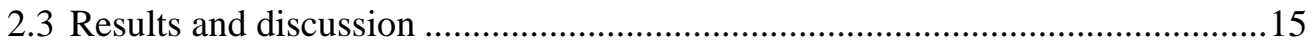

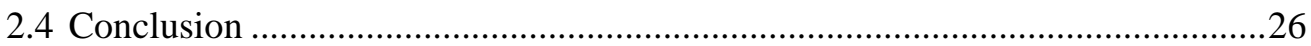

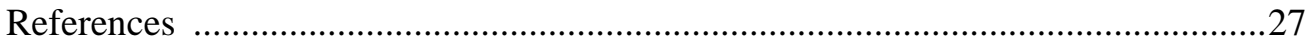

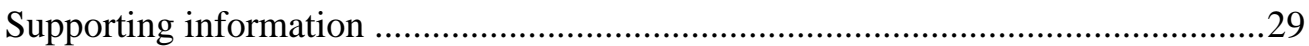

\section{Chapter 3}

Large-area high-contrast hydrophobic/hydrophilic patterned Surface for Robust Electrowetting Devices ......................................................................................31

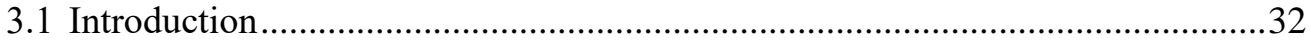

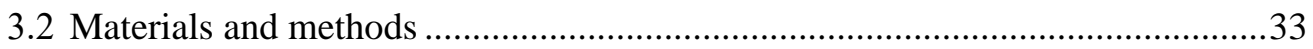

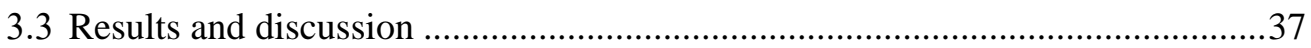

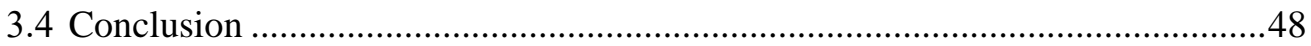

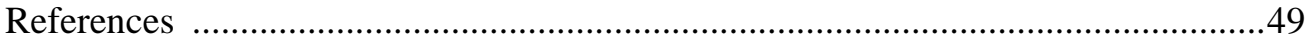

\section{Chapter 4}

Electrically controlled localized charge trapping at amorphous fluoropolymer-

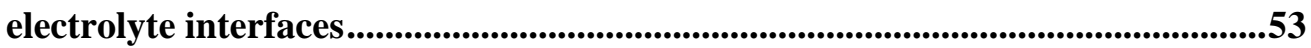

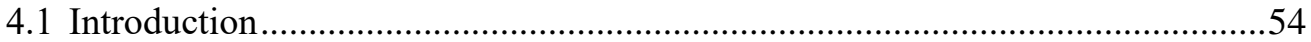

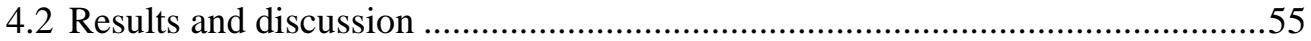




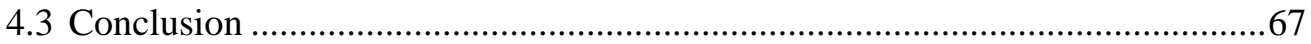

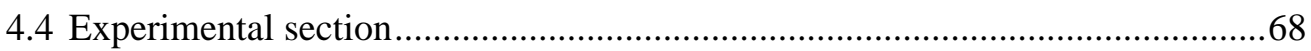

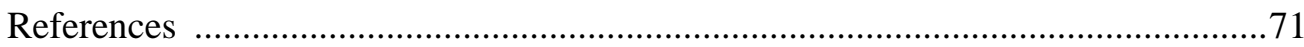

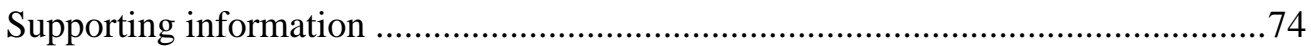

\section{Chapter 5}

Electric power generation from drop impacting on charged surfaces ................85

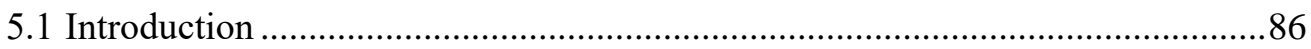

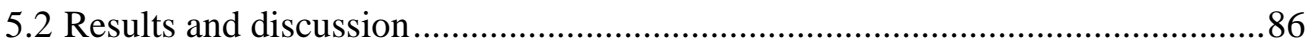

5.3 Sample preparation and solid-liquid area measurement ....................................95

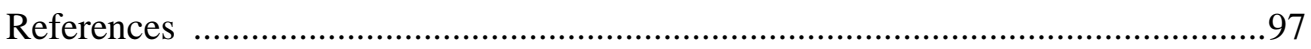

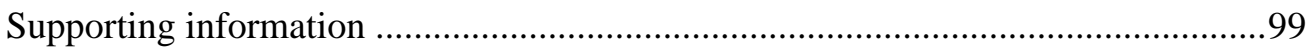

\section{Chapter 6}

Why and how to achieve ultrahigh surface charge density for charge trapping electric nanogenerator (CT-ENG) ....................................................................113

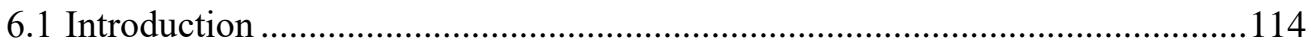

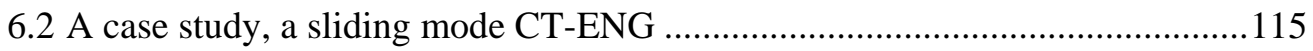

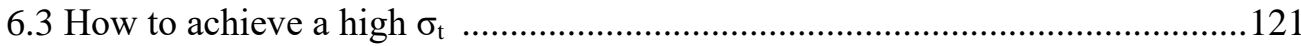

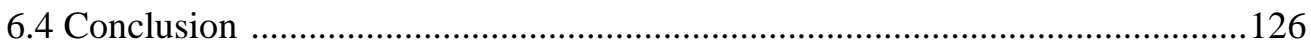

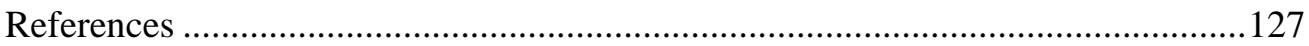

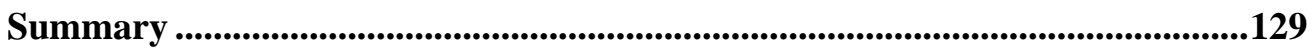

Samenvatting..................................................................................................................131

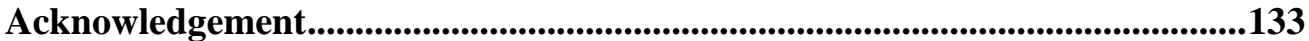

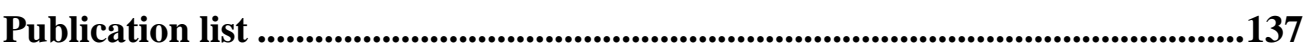

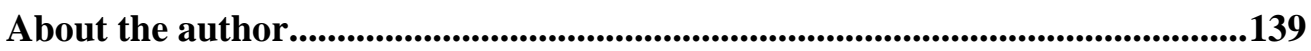




\section{Chapter 1}

\section{Introduction}

This chapter provides a brief introduction of this thesis. In this thesis, we will discuss two kinds of responsive systems based on fluoropolymers. One is "electrowetting", an electrical responsive system with input stimulus of electricity and output response of liquid movement. The other one is "charge trapping electric nanogenerator (CTENG)", an electrically responsive system with input stimulus of liquid motion and output response of electric power. Two typical applications of electrowetting displays and energy harvesting from water motion will be mainly discussed. 


\subsection{Responsive systems}

Responsive systems refer to the systems which can response to a certain stimulus or more than one stimulus. These stimuli could be electrical, light, thermal, contact, etc. The response of the systems could also be in many different forms, such as mechanical, electrical, chemical , etc.. The schematic representation of such systems can be seen in Figure 1.1.

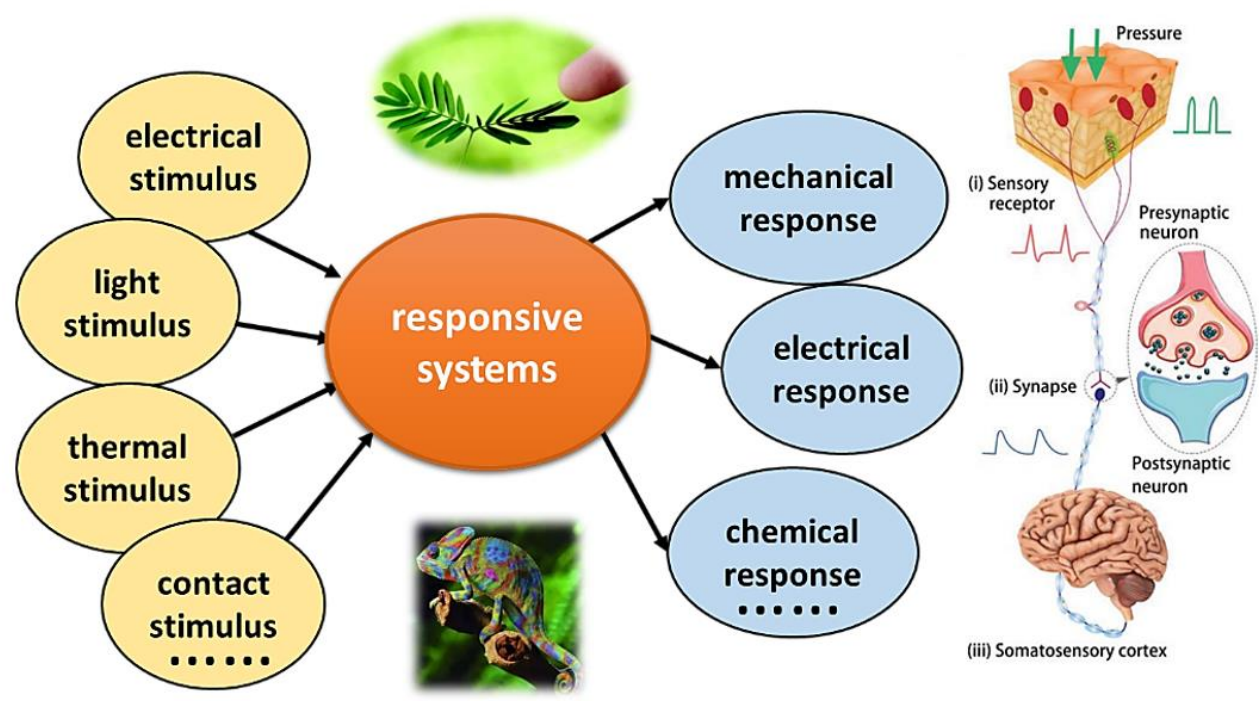

Figure 1.1 Various types of responsive systems. The illustration of biological haptic perception system is from reference ${ }^{[1]}$.

Such responsive systems can be easily found in nature. For example, leaves of Mimosa pudica are able to respond to several stimuli, like touching, vibration and photo stimulation $^{[2-4]}$. Chameleon's skin can respond to and match the colors from the environment accordingly ${ }^{[5]}$. There are also extensive and more complex responsive systems in human beings' physiological systems, such as sensory neuron ${ }^{[6]}$, to ensure that we can react properly to the stimuli from the internal physical systems and the external environment. These systems look diverse, but their essences, or at least of the first step of these responsive processes, are actually same. They all convert one kind of 'signal' into another 'signal' in the broadest sense. These "signals" could be a mechanical movement, a transformation, an electrical current, a chemical reaction, or 
an energy or enthalpy change. The existence and interactions of these numerous elegant responsive systems make our universe functional, active, and splendid.

\subsection{Electrically responsive fluoropolymer surfaces}

In this thesis, we study electrically responsive systems based on fluoropolymer surfaces. There are two aspects according to this topic (Figure 1.2). One is, the surfaces 'response to electricity', which means the surface properties, mostly wettability in this thesis, change with the applied electric field (the electric signals). This phenomenon is known as 'electrowetting (EW)'. In this scenario, the electricity is an input signal and the process is an electro-mechano-transduction. The other one is the surfaces 'response in form of electricity', which refers to that a fluoropolymer surface receives a mechanical stimulus and converts this mechanical stimulus into an electric signal. In this situation, the electricity is an output signal, and the process is a mechano-electro-transduction.

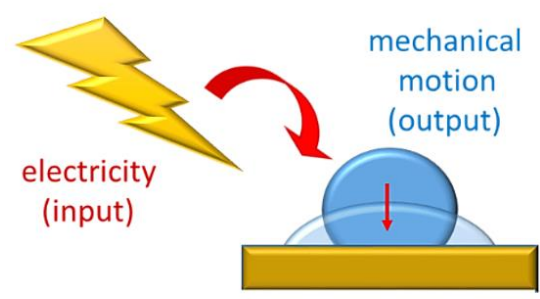

Response to electricity (electro-mechano-transduction)

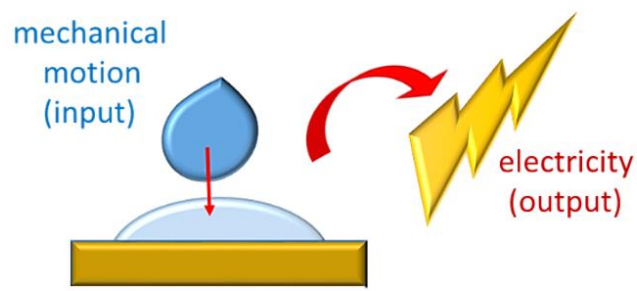

Response in electricity (mechano-electro-transduction)

Figure 1.2 Two electrically responsive systems based on fluoropolymer surfaces in this thesis.

\subsubsection{Fluoropolymer surface responding to electricity}

Electrowetting- a system of surface wettability responding to electricity

The properties of a surface can be varied by many approaches. EW is an approach to attain electrically responsive surfaces. By using EW, the wettability of hydrophobic surfaces can be reversibly switched without changing their chemical composition. ${ }^{[7]}$

Electrocapillarity, the basis of modern electrowetting, was first described in detail in $1875^{[8]}$. In the early $1990 \mathrm{~s}$, Berge introduced the idea of using a thin insulating layer 
to separate the conductive liquid from the metallic electrode in order to eliminate the problem of electrolysis ${ }^{[9-10]}$. To emphasize the relevance of the dielectric layer, EW is often also denoted as 'electrowetting-on-dielectric' (EWOD). Recently, it has attracted many attention because of its broad applications in microfluidics ${ }^{[11-12]}$, optofluidics $^{[13-14]}$, display technology ${ }^{[15]}$.

The working principle of EWOD is schematically shown in Figure 1.3. When a voltage $(\mathrm{V})$ is applied on the dielectric layer via an electrolyte droplet and the bottom electrode, a pulling force, emerging from the applied electric field, pulls the three phase contact (TPCL) line towards the outward direction of the droplet, and thus changes the contact angle. The contact angle $\theta(\mathrm{V})$ is given by the classical YoungLippmann EW model:

$$
\cos \theta(V)=\cos \theta_{Y}+\frac{c}{2 \gamma} V
$$

where $\theta_{\mathrm{Y}}$ is Young's angle, $\gamma$ is the surface tension of the liquid and $\mathrm{V}$ is the applied voltage.
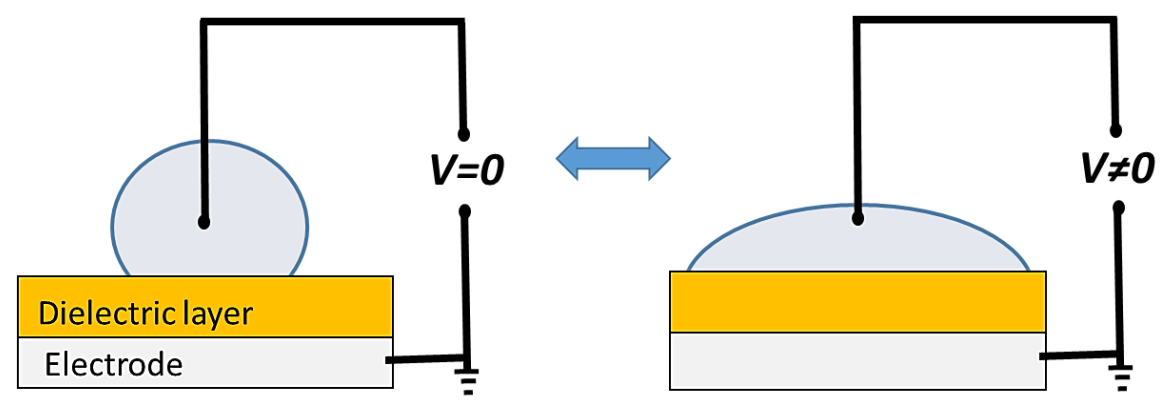

Figure 1.3 Illustration of the electrowetting on dielectric (EWOD) principle.

Fluoropolymer- a suitable material for reversible EW

A material system with low contact angle hysteresis is essential to make a switchable EW device. EW always requires the motion of contact lines by definition. Therefore, it is naturally affected by contact angle (CA) hysteresis. As shown in Figure 1.4, a water droplet typically exhibits an advancing contact angle $\left(\theta_{a}\right)$ prior to initial application of the voltage. When the voltage is applied, the liquid advances further to $\theta_{a, V}$. When the voltage is removed the liquid recedes to $\theta_{r}$. A further switching on and off of the voltage leads to a transition between the receding angle, $\theta_{r}$, in the 
absence of a voltage and the advancing angle, $\theta_{a, v}$, in the presence of the voltage. So reversible switching is only possible when the contact angle hysteresis is low, and $\theta_{\mathrm{r}}$, exceeds $\theta_{a, V}$. On most solids, this is not the case, meaning that only a single switch is possible, with no reversibility. The modulation of a reversible electrowetting device is given by $\theta_{r}-\theta_{a, V}$.

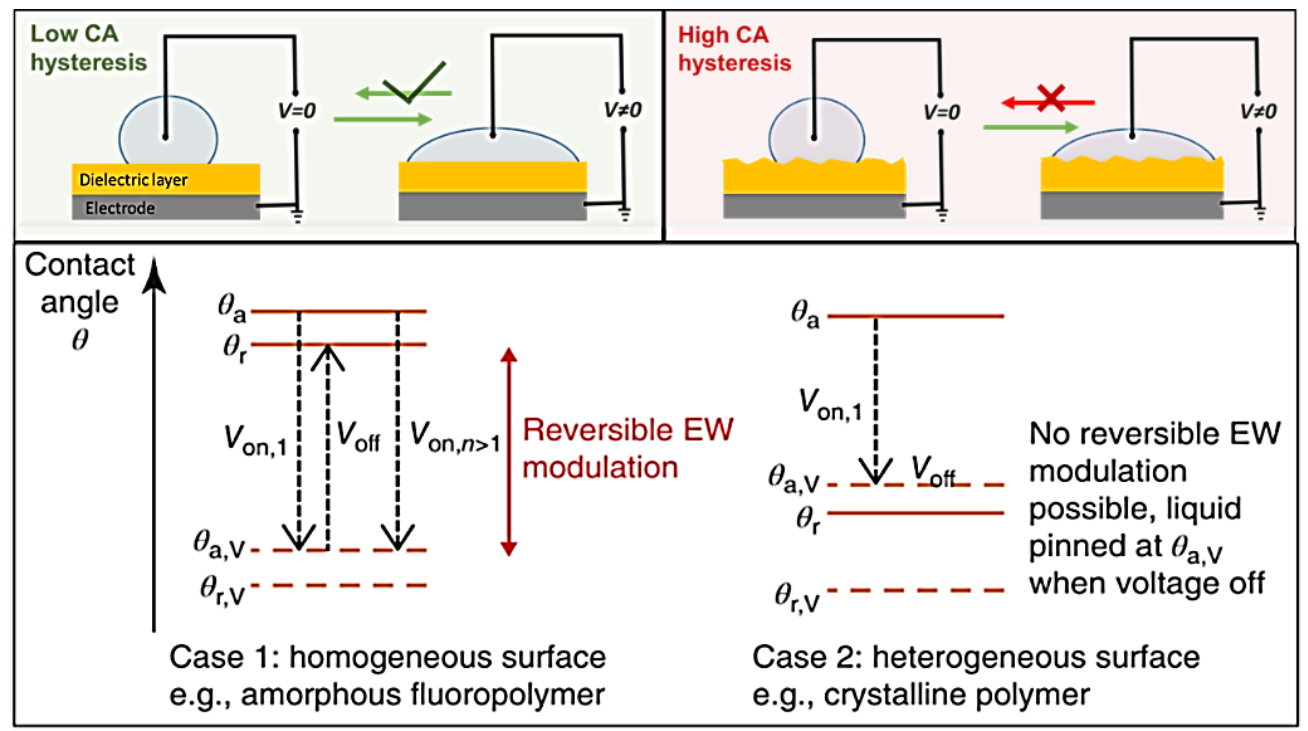

Figure 1.4 Effect of surface contact angle (CA) hysteresis on reversibility of electrowetting.

As a result, amorphous fluoropolymer (AFP), as a kind of hydrophobic material with low contact angle hysteresis, is preferred in EW devices. The typical and most studied amorphous fluoropolymers are Teflon AF and Cytop. These two matrials are also the main materials utilized in the research presented in this thesis. Teflon AF was previously produced by DuPont company, and this product is currently provided by the Chemourse company, a spin-off company from DuPont. Cytop matreial is provided by AGC Chemicals company. Hyflon is also an amorphous fluoropolymer material produced by Solvay company.

\subsubsection{Fluoropolymer surface response in form of electricity}

Some of the materials, when receiving stimuli on their surfaces, can respond and generate a detectable electrical signal. Such examples can be found in material classes 
of piezoelectric , thermoelectric, photosensitive and ferroelectric ones. In this thesis, we discuss an phenomenon that the electric current signal can be generated when a water drop moves on a fluoropolymer surface with trapped charges. This current signal was initially found while monitoring the leakage current during electrowettinginduced charge trapping experiment. After further systematical investigation, this electric current signal was proved to be reproducible and this phenomenon was utilized as an electrical nanogenerator for harvesting energy from water droplets. The 'nano-'generator, refers to the electrical generation system that can convert nanoscale mechanical energy to an electrical response ${ }^{[16]}$.
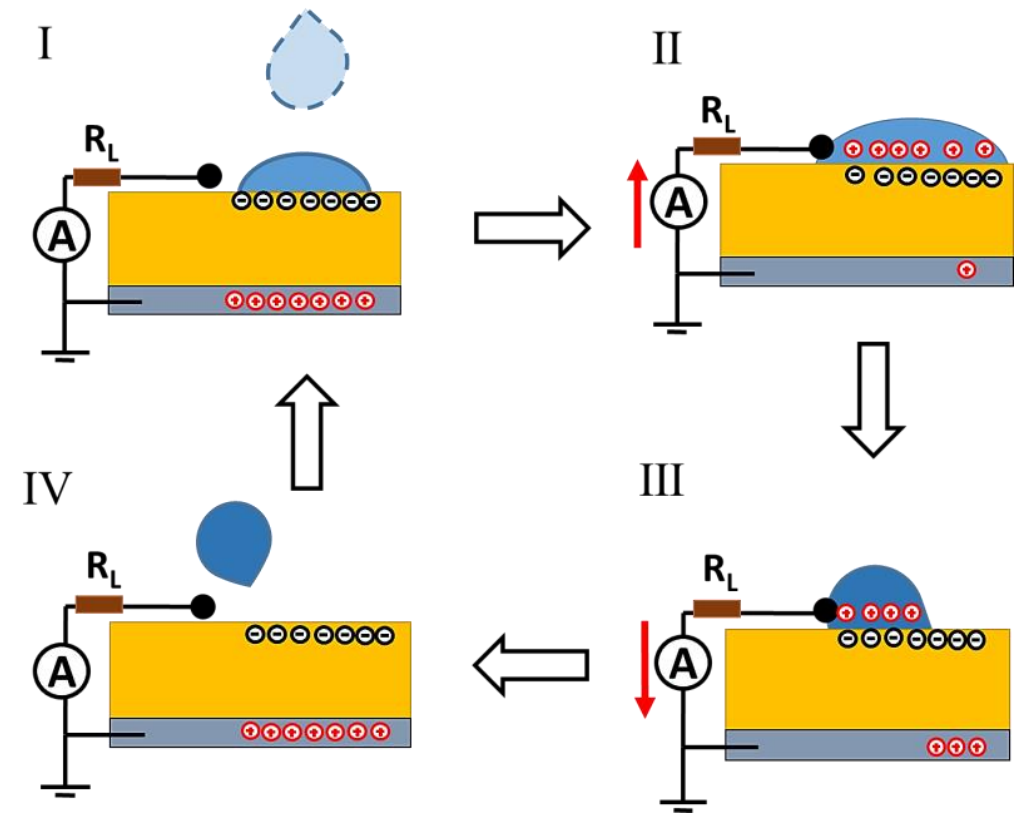

Figure 1.5 Schematic of the mechanism of CT-ENG (charge trapping electrical nanogenerator)

In electrowetting applications, charge trapping is actually a long-standing problem. It was reported frequently that during operating EW devices, the charges can be trapped in the fluoropolymer films and cause the contact angle saturation and device reliability degradation ${ }^{[17-18]}$. In this thesis, we first investigate how the charges could be trapped at fluoropolymer surfaces by EW. It is found that the charges are accumulated to the TPCL region after EW process, because the local electric field at TPCL region is much higher compared to that of other regions. While a drawback for most conventional EW applications, we demonstrate this EW-assisted Charge Injection (EWCI) can serve as a simple and low-cost method to deposit stable charges on fluoropolymers. 
Following this discovery, by introducing a dielectric layer with high dielectric strength, we are able to deposit charges with relatively high density on a centimeter sized fluoropolymer surface. Based on this surface with trapped charges, we have built a charge trapping electric nanogenerator setup (CT-ENG) to harvest energy from water motions.

The working principle of CT-ENG is illustrated in Figure 1.4. In general, the electric generating process can be described in 4 steps. Step 1: The droplet contacts and spreads on the charged surface. Before the droplet touches the top electrode, the (positive) counter charges are in the bottom electrode layer, therefore, no current is generated. Step 2: The droplet touches the top electrode, and negative current is generated due to the migration of counter charges from bottom electrode to the top electrode. Step 3: The droplet keeps in contact with the top electrode and positive current is generated as the liquid/solid interfacial area decreases. Counter charges transfer back to the bottom electrode. Step 4: The droplet leaves the top electrode and all charges move back in the bottom electrode layer again. Further details of the CTENG will be discussed in Chapter 5. In the last part of this thesis, we further enhance the surface charge density by optimizing the charging condition and the dielectric film construction. Charge density as high as $1.8 \mathrm{mC} / \mathrm{m}^{2}$ and energy harvesting efficiency of $10 \%$ are thus achieved.

\subsection{Aim of the thesis}

The aim of the thesis is to investigate how do the amorphous fluoropolymers work in the electrically responsive systems, and how to improve the performance of the related devices. EW display devices and electrical nanogenerators are used as two platforms to implement the research.

\subsection{Outline of the thesis}

The outline of this thesis is as follows. In Chapter 2, we investigate the influence of the amorphous fluoropolymer in EW display devices, and three typical amorphous fluoropolymers, Teflon AF, Cytop, and Hyflon, are studied in this chapter. We reveal that even slight lacking of hydrophobicity of fluoropolymer surfaces will lead to the "non-closing" failures in the EW display devices. In Chapter 3, an approach of fabricating hydrophobic/hydrophilic surfaces with high wettability contrast is proposed for manipulating water motions in a micro-sized confined region. The 
surface damage of fluoropolymer during the fabrication process is successfully avoided with the proposed approach. EW display devices are utilized as an example to verify the advantage of this approach. In Chapter 4, we investigate the charge trapping phenomenon in EW. We find that the trapped charges are accumulated in the TPCL region, which means the failures in EW may also occurs in the TPCL region. However, in another view point, these findings also provide a simple and an efficient method for fabricating surface charge pattern. Macro-scale charge regions are fabricated by such an electrowetting assisted charge injection (EWCI) method. In Chapter 5, we continue the exploration of the utilization of charge trapping by using EWCI method. By introducing $\mathrm{SiO}_{2}$ layer with a high dielectric strength and protecting the TPCL region, we are able to apply higher voltages on the AFP films. Therefore, trapped charges can be deposited on a larger area of AFP surfaces. We investigate the mechanism of electrical responses generated from the charged AFP surfaces, and propose a charge trapping electrical nanogenerator (CT-ENG) for energy harvesting from water motions. The performance of CT-ENG is also investigated in this chapter. In Chapter 6, we discuss the influencing factors of CTENG and propose an optimized EWCI approach to enhance the trapped charge density of CT-ENG, and thus to improve the performance of CT-ENG.

\section{Contributions}

Hao $\mathrm{Wu}$ wrote this chapter. Frieder Mugele and Guofu Zhou provided suggestions. Hao $\mathrm{Wu}$ and Beybin Ilhan revised this chapter.

\section{References}

[1] C. Zhang, W. B. Ye, K. Zhou, H. Y. Chen, J. Q. Yang, G. Ding, X. Chen, Y. Zhou, L. Zhou, F. Li, Advanced Functional Materials 2019, 29, 1808783.

[2] A. G. Volkov, J. C. Foster, T. A. Ashby, R. K. Walker, J. A. Johnson, V. S. Markin, Plant, cell \& environment 2010, 33, 163.

[3] M. Weintraub, The New Phytologist 1952, 50, 357.

[4] J. Fondeville, M. Schneider, H. Borthwick, S. Hendricks, Planta 1967, 75, 228.

[5] H.-H. Chou, A. Nguyen, A. Chortos, J. W. To, C. Lu, J. Mei, T. Kurosawa, W.-G. Bae, J. B.-H. Tok, Z. Bao, Nature communications 2015, 6, 8011. 
[6] D. Usoskin, A. Furlan, S. Islam, H. Abdo, P. Lönnerberg, D. Lou, J. HjerlingLeffler, J. Haeggström, O. Kharchenko, P. V. Kharchenko, Nature neuroscience 2015, 18, 145.

[7] F. Mugele, J. Heikenfeld, Electrowetting: Fundamental Principles and Practical Applications, John Wiley \& Sons, 2018.

[8] G. Lippmann, Gauthier-Villars Paris, France:, 1875.

[9] F. Mugele, J.-C. Baret, Journal of physics: condensed matter 2005, 17, R705.

[10] B. Bruno, Comptes Rendus de L'Academie des Sciences Paris, Serie, II 1993, 317, 157.

[11] M. G. Pollack, R. B. Fair, A. D. Shenderov, Applied Physics Letters 2000, 77, 1725.

[12] S. K. Cho, H. J. Moon, C. J. Kim, Journal of Microelectromechanical Systems 2003, 12, 70 .

[13] B. Berge, J. Peseux, European Physical Journal E 2000, 3, 159.

[14] K. Mishra, D. van den Ende, F. Mugele, Micromachines 2016, 7.

[15] R. A. Hayes, B. J. Feenstra, Nature 2003, 425, 383.

[16] Z. L. Wang, J. Song, Science 2006, 312, 242.

[17] X. Li, H. Tian, J. Shao, Y. Ding, X. Chen, L. Wang, B. Lu, Advanced Functional Materials 2016, 26, 2994.

[18] T. Krupenkin, J. A. Taylor, Nature communications 2011, 2, 448. 


\section{Chapter 2}

\section{Influence of fluoropolymer surface wettability on electrowetting display performance*}

Amorphous fluoropolymer (AFP), as a material for both insulating and hydrophobic coating, plays an essential role in electrowetting displays (EWD). In this chapter, three AFPs are studied according to their influences on the EWD performances. Reversible and fast optical switch could be achieved in the EWD devices fabricated using all three AFPs; however, less hydrophobicity of the Cytop 809A surface would lead to a slower off-switching speed and even incomplete close of the micro-pixels in EWDs. The "reflow" temperature for restoring the hydrophobicity of fluoropolymer surface should be high enough to achieve a sufficient surface recovery, and at the same time avoid inducing failures like film dislocation and breakdown. The optimal "reflow" temperature has been investigated and evaluated based on the EWD performances.

\footnotetext{
${ }^{*}$ This chapter is based on publication: H. Wu, R.A. Hayes, F. Li, A. Henzen, L. Shui, G. Zhou, Influence of fluoropolymer surface wettability on electrowetting display performance, Displays, 53 (2018) 47-53.
} 


\subsection{Introduction}

Electrowetting has been widely used to manipulate liquid motion at small scales. It was first exploited by Lippmann in $1875^{[1]}$. Recently, it has attracted a lot of attentions because of its broad applications in electrowetting displays (EWD ${ }^{[2-4]}$, digital microfluidics $^{[5]}$, lenses ${ }^{[6]}$, and energy harvesting ${ }^{[7]}$.The working principle of electrowetting on dielectric (EWOD) can be described by Young-Lippmann's equation $^{[8]}$

$$
\cos \theta-\cos \theta_{0}=\frac{\varepsilon_{0} \varepsilon_{r}}{2 d \gamma} U^{2}
$$

where $\theta_{0}$, the initial contact angle; $\gamma$, the interfacial tension between two fluids; $\varepsilon_{0}$, the vacuum permittivity; $\varepsilon_{\mathrm{r}}$, the relative permittivity of the insulator; $d$, the thickness of the insulator and $U$ is the applied voltage. As can be seen from the equation, the insulator and the surface wettability (contact angle) are key parameters for electrowetting performance. It has also been proven that the insulating layer and the hydrophobic coating is very important for electrowetting performance, and therefore the related parameters, including driving voltage, degradation of electrowetting effect and leakage current ${ }^{[9-13]}$. The combination of inorganic thin film as insulating layer and fluoropolymer (FP) as hydrophobic top coating has been widely used to investigate the electrowetting phenomenon. The inorganic insulator materials (such as $\mathrm{SiO}_{2}, \mathrm{TiO}_{2}, \mathrm{Si}_{3} \mathrm{~N}_{4}$, and so on) with high dielectric constant were normally used to decrease the electrowetting actuating voltage based on the Young-Lippmann's equation $^{[10-12]}$. Teflon AF1600 and Cytop 809A have been commonly used as hydrophobic top layer because of their low surface energy ${ }^{[10-14]}$. It was reported that Cytop showed superior long-term electrowetting on dielectric (EWOD) performance compared with Teflon $\mathrm{AF}^{[13]}$.

Except for EWOD, electrowetting on liquid-infused films (EWOLF) has recently received increasing interest. Dielectric liquid lubricants are spread on the surface and being locked in a membrane to form a smooth liquid-infused dielectric layer which could minimize the contact angle line pinning and lead to fast response without sacrificing the desired electrowetting reversibility. EWOLF has been applied for complete reversibility and controlled droplet oscillation suppression in droplet electrowetting devices ${ }^{[15-16]}$. 
EWD is a device utilizing a dual fluidic system of colored oil and transparent water to realize display function in a microscale pixel based on electrowetting mechanism. The concept of EWD was first proposed by Beni et al. ${ }^{[17]}$. In 2003, the functional EWD device was reported by Hayes et $\mathrm{al}^{[2]}$. Afterwards, a lot of research has been done to optimize the EWD performance from the views of materials, fabrication process or electrical control ${ }^{[18-21]}$. Figure 2.1 shows the schematic drawing of a EWD pixel at "off" (Figure 2.1a) and "on" (Figure 2.1b) states. In the absence of a voltage, the oil forms a continuous film in a pixel between the hydrophobic insulator-covered electrode and water, showing the color of the oil film. When a voltage is applied across the top and bottom electrodes, the transparent water is driven to move towards the insulator, pushing the oil film aside or break, showing the color of the bottom substrate. In this way, the optical properties of the stack, when viewed from the top, are tuned between a colored off-state (dyed oil) and a white On-state (color of bottom substrate).

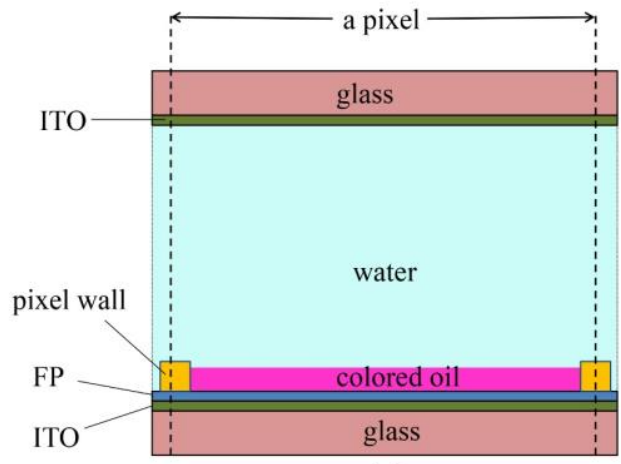

(a)

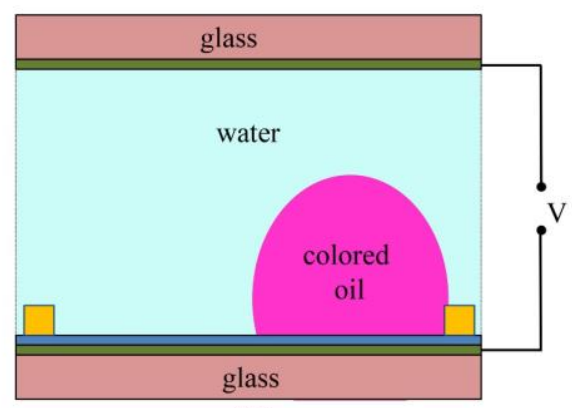

(b)

Figure 2.1 Schematic drawing of an electrowetting display (EWD) pixel. (a) Off state: in a EWD pixel, without applied voltage, a homogeneous oil film spreads over the pixel area showing the color of the dyed oil. (b) On state: in a EWD pixel, with an applied voltage of V, the oil film was pushed by water to one corner of the pixel, showing the color of the bottom substrate.

Recently, FPs have been widely applied in electrowetting devices as both insulating and hydrophobic layer ${ }^{[3,9,18-19]}$. The breakdown voltage as high as $100 \mathrm{~V} / \mu \mathrm{m}$ has been achieved for Teflon AF1600 when its thickness was lower than $1.0 \mu \mathrm{m}^{[9]}$. Large scale EWD devices have also been processed with both Teflon AF1600 and Cytop 809A 
materials ${ }^{[3,20]}$. However, the comparison among different FP's influence on EWD performance has not been reported yet.

In this chapter, three FPs based on Teflon AF1600, Hyflon AD60 and Cytop 809A are investigated according to their effects on the EWD performance. The surface wettability of FPs is evaluated by the contact angles of the water droplet in air. To mimic the real situation in an electrowetting display device, the contact angles of the oil droplet in water surrounding are introduced. Two specific processes, named "activation" and "reflow" in EWD fabrication, could be achieved by reactive ion etching (RIE) and thermal annealing. The contact angle changes before and after such processes have been investigated to understand the surface wettability change, and thus their effect on EWD performance. Therefore, an optimum "reflow" temperature for each FP has been obtained and used for EWD fabrication. Moreover, the failure modes caused by annealing at high temperature are also studied to understand the reasons of device damage.

\subsection{Materials and Methods}

\subsubsection{Materials}

Commercial indium tin oxide (ITO) glass with thickness of $0.7 \mathrm{~mm}$ and resistance of $100 \Omega / \square$ was purchased from Guangdong Jimmy Glass Technology Ltd. (Foshan, China). Amorphous fluoropolymer based on Teflon AF1600 (Dupont, Shanghai, China), Hyflon AD60 (Solvay, Shanghai, China) were dissolved into FC-43 (Minnesota Mining \& Manufacturing Company, Saint Paul, USA) with concentration of $3.7 \mathrm{wt} \%$ and $6.5 \mathrm{wt} \%$, respectively. Cytop 809A solution was purchased from Asahi Glass Co., Ltd (Kanagawa, Japan) with concentration of $9.0 \mathrm{wt} \%$. Negative photoresist for fabricating pixel walls was co-developed with a local material supplier. The conductive liquid was $1.0 \mathrm{mM} \mathrm{NaCl}$ solution with conductivity of $\sim 110 \mu \mathrm{S} / \mathrm{cm}$. The color dye was designed and synthesized in our lab. Decane (Micklin, Shanghai, China) was used as dye solvent. The interfacial tension of colored oil (0.21 M dye decane solution) / conductive liquid (1.0 $\mathrm{mM} \mathrm{NaCl}$ aqueous solution) was $19 \mathrm{mN} / \mathrm{m}$. 


\subsection{Device Fabrication}

ITO glass was used as the starting substrate which was initially cleaned in a cleaning line (KJD-7072ST, KEJINGDA Ultrasonic Equipment Co., Ltd., Shenzhen, China), and then coated with amorphous fluoropolymers using a spin coater (KW-5, Institute of Microelectronics Chinese Academy of Sciences, Beijing, China) at the speed of 1000 - $2000 \mathrm{rpm}$ for $60 \mathrm{~s}$. FP coating was then dried on a hotplate at $85^{\circ} \mathrm{C}$ for $5 \mathrm{~min}$ and then in an oven at $185{ }^{\circ} \mathrm{C}$ for 2 hours, obtaining $~ 800 \mathrm{~nm}$ thick fluoropolymer film on the ITO-glass. In order to coat the photoresist on it, the FP surface was treated to hydrophilic by using a reactive ion etching (RIE) machine (ME-6A, Institute of Microelectronics Chinese Academy of Sciences, Beijing, China) with slight oxygen plasma ( $5 \mathrm{~W}$ for $10 \mathrm{~s}$ plasma treatment). Photoresist was coated on the FP surface, and lithography process was applied using an aligner (URE-2000/35, Institute of Optics and Electronics, Chinese Academy of Sciences, Chengdu, China) to make the pixel walls. A thermal reflow process was applied by putting the substrate in an oven (5FG01B, Huangshi, China) under a certain degree for $2 \mathrm{~h}$. Afterwards, the ITO-glass with FP layer and pixel walls was filled with colored oil, assembled and sealed with a bare ITO-glass under water. The detailed process has been described in the reference ${ }^{[20-21]}$.

\subsubsection{Measurements}

Contact angle was measured using a Contact Angle Meter (POWEREACH, Shanghai Zhongchen Digital Technology Apparatus Co., Ltd. Shanghai, China). Thickness and surface morphology of the FP and pixels were measured using a stylus profiler (Dektak XT, BRUKER Corporation, Shanghai office, China). A waveform generator (Agilent 33500B Series, Santa Clara, CA, USA) and an amplifier (Agilent 35502A) were used to provide square wave signals with specific voltage amplitude to drive the EWD devices. An optical colorimeter (Arges 45, Admesy, Ittervoort, the Netherlands) was used to measure the optical response of the devices. The incident light was shined at an angle of $45^{\circ}$, and a detector at $45^{\circ}$ angle with surface area of $\sim 1 \mathrm{~cm}^{2}$ was positioned on the device area. Optical microscope (CTX41, Olympus, Tokyo, Japan) equipped with a high-speed camera (Phantom MiRO M110, Wayne, USA) was used to visualize and record the oil movement in the devices. The scanning electron microscope (SEM) (ZEISS Ultra 55, Carl Zeiss, Jena, Germany) was used to observe the sample structures. 


\subsection{Results and discussion}

\subsubsection{Effect of surface wettability on switching speed}

Cytop is a commonly used fluoropolymer coating for electrowetting systems ${ }^{[13-14,20]}$. It showed superior long-term electrowetting on dielectric (EWOD) performance in comparison with other FP materials ${ }^{[13]}$. For EWD application, the reversibility and switching speed are key factors for device evaluation. Since FP surface directly contacts with the hydrophobic oil and conductive water, the surface properties play the key role in electrowetting performance. Surface roughness is commonly assumed to be similar after a standard coating and thermal treatment process. However, the surface wettability varies with chemical components of the FP materials. In this work, the three FP materials based on Teflon AF1600, Hyflon AD60 and Cytop 809A were compared (Table S1). The FP films were fabricated using the process described in the fabrication section. Figure 2.2 shows the contact angle (CA) measurements on the three FP coatings. There was only slightly difference in the contact angles of waterin-air (W/A) $\left(\theta_{(\mathrm{W} / \mathrm{A})}\right)$ on the three FP coatings. The advancing contact angle $\left(\theta_{\mathrm{Adv}(\mathrm{W} / \mathrm{A})}\right)$ on Teflon AF1600, Hyflon AD60 and Cytop 809A surfaces were 120, 120 and $116^{\circ}$, respectively, as shown in Figures 2.2a,2.2b and 2.2c; and the receding contact angle $\left(\theta_{\operatorname{Rec}(\mathrm{W} / \mathrm{A})}\right)$ were 110,110 and $102^{\circ}$, respectively, as shown in Figure $2.2 \mathrm{~d}, 2.2 \mathrm{e}$ and 2.2f. The contact angle difference may be attributed to the different distribution of fluorine-containing groups in polymer structures (Table S1). In addition to difluoro $\mathrm{CF}_{2}$ - groups, Teflon AF1600 and Hyflon AD60 contain trifluoro $-\mathrm{CF}_{3}$ or $\mathrm{OCF}_{3}-$ groups; however, Cytop 809A is composed mainly of difluoro $-\mathrm{CF}_{2}$ - groups.

In EWD devices, the existed bi-fluidic system is the dyed oil solution and the conductive aqueous solution. Therefore, the contacts of either water droplet surrounded by oil (W/O) or oil droplet surrounded by water (W/O) should be investigated. Here, the oil-in-water $(\mathrm{O} / \mathrm{W})$ contact angle $\left(\theta_{(\mathrm{O} / \mathrm{W})}\right)$ was measured, as shown in Figure 2.2(g-i). $\theta_{(\mathrm{O} / \mathrm{w})}$ on Teflon AF1600 and Hyflon AD60 were smaller than $10^{\circ}$ (Figure $2.2 \mathrm{~g}$ and $2.2 \mathrm{~h}$ ), while the $\theta_{(\mathrm{O} / \mathrm{w})}$ on Cytop 809A was higher than $40^{\circ}$ (Figure 2.2i). This means that the surfaces of Teflon AF1600 and Hyflon AD60 showed obviously higher affinity to the oil phase compared to Cytop 809A surface. In other words, the $\theta_{(\mathrm{O} / \mathrm{w})}$ was more sensitive than $\theta_{(\mathrm{W} / \mathrm{A})}$ regarding to the characterization of the surface wettability. The $\theta_{(\mathrm{O} / \mathrm{w})}$ difference was $>40^{\circ}$ which would induce big variation in electrowetting phenomenon. 


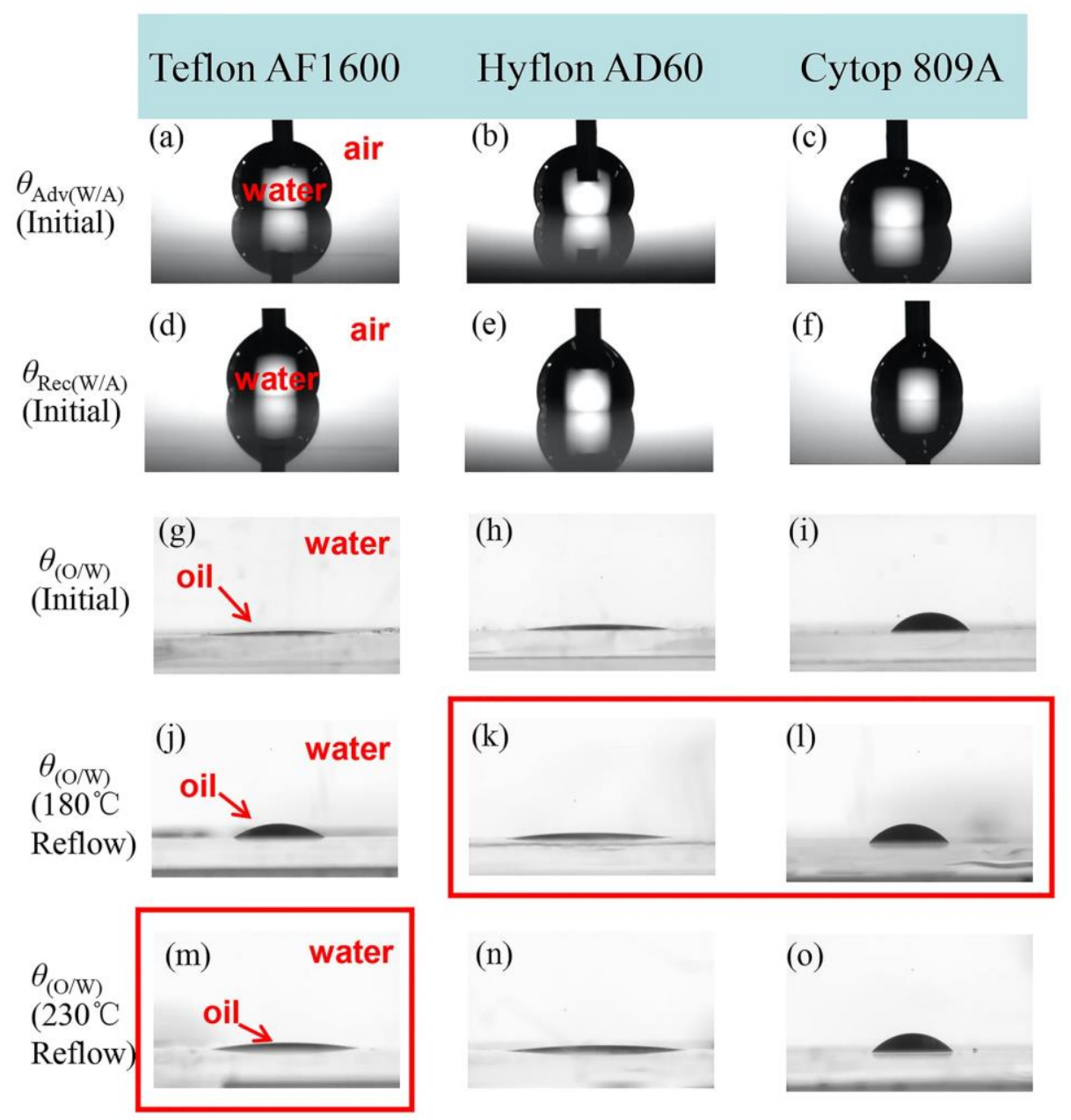

Figure 2.2 The advancing and receding contact angle of water droplet on initial FP coatings of Teflon AF1600 (a, d), Hyflon AD60 (b, e) and Cytop 809A (c, f). The contact angles of the oil droplet surrounded by water on the initial coatings of Teflon AF1600 (g), Hyflon AD60 (h) and Cytop 809A (i). The contact angles of the oil droplet surrounded by water on the coatings after "reflow" treatment: Teflon AF1600 (j, m), Hyflon AD60 (k, n) and Cytop 809A (l, o). The oil volume in (g)-(o) is $0.5 \mu \mathrm{L}$.

To fabricate a EWD device, the FP film needs to be treated to be hydrophilic for photoresist coating to fabricate pixels on it ${ }^{[3,18]}$. The FP surfaces were "activated" by 
using a RIE process to be hydrophilic, and then recovered using a thermal annealing process called "reflow" to treat it back to hydrophobic. Therefore, the contact angles of water droplets on the FP surfaces after "reflow" were also measured. The "reflow" temperature is typically set at above the phase transition temperature $\left(T_{\mathrm{g}}\right)$ of the FP. Figure $2.2 \mathrm{~d}$ and $2 \mathrm{e}$ show $\theta_{(\mathrm{O} / \mathrm{w})}$ on FP surfaces after "reflow" at 180 and $230{ }^{\circ} \mathrm{C}$, respectively. It is seen that the Cytop 809A film surfaces showed less affinity to oil compare to those of Teflon AF1600 and Hyflon AD60 films. Hyflon AD60 and Cytop 809 A were both recovered to their initial hydrophobicity after "reflow" at 180 and $230{ }^{\circ} \mathrm{C}$. However, Teflon AF1600 only recovered partially after $180{ }^{\circ} \mathrm{C}$ "reflow" treatment $\left(\theta_{(\mathrm{O} / \mathrm{w})} \approx 30^{\circ}\right)$, and completely recovered after $230^{\circ} \mathrm{C}$ treatment $\left(\theta_{(\mathrm{O} / \mathrm{w})} \approx 10^{\circ}\right)$. This means that FP materials need to be treated at higher temperature to restore its surface to original hydrophobicity according to its higher $T_{\mathrm{g}}$.

To investigate the surface wettability effect on the EWD performance, the devices composed of the three FPs as hydrophobic insulating layers were fabricated. Based on the $\theta_{(\mathrm{O} / \mathrm{w})}$ results, the reflow temperatures were set at 230,180 and $180{ }^{\circ} \mathrm{C}$, for Teflon AF1600, Hyflon AD60 and Cytop 809A, respectively. Figure 2.3a is a photograph of the samples with display area of $50.7 \times 66 \mathrm{~mm}^{2}$. Figures $2.3 \mathrm{~b}$ and $3 \mathrm{c}$ show the fabricated pixel walls on the FP surface with width of $15 \mu \mathrm{m}$ and height of $5.8 \mu \mathrm{m}$. The pitch of the pixels was $150 \mu \mathrm{m}$. To test the switching behavior of the EWD devices, $30 \mathrm{~V}$ voltage difference was applied to the ITO electrodes on the upper and lower substrates. Typical "off" and "on" states were displayed for all three EWD devices, as shown in Figures 2.3d and 3e. At the "off" state, the colored oil spread on the FP surface, showing the color of the oil phase. At the "on" state, the oil film was pushed aside by the conductive water phase because of the electrowetting effect, showing the color of the bottom substrate. 

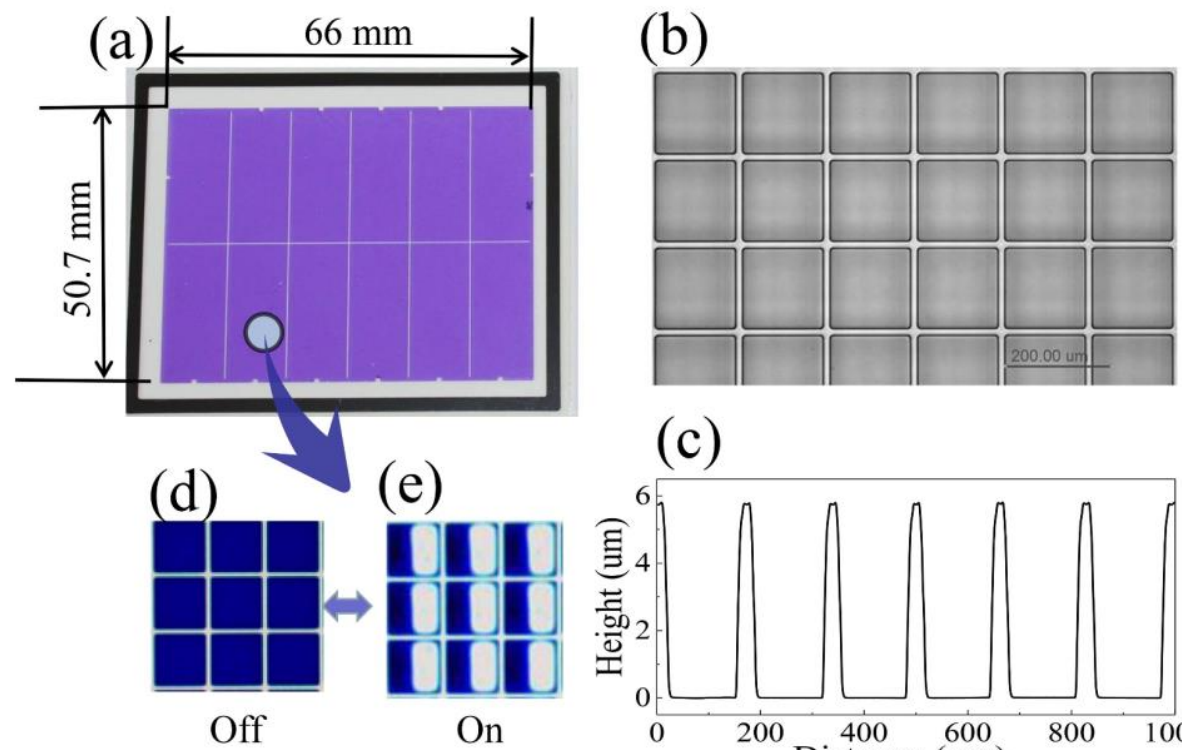

\section{(c)}

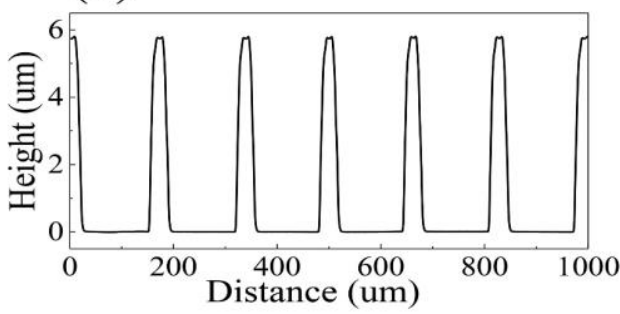

Figure 2.3 Electrowetting devices: (a) optical image of a fabricated EWD device, (b) the microscopic image of pixels, (c) the surface profile measurement of the pixels on a FP surface, (d) "Off" state of 9 pixels in the EWD device, and (e) "On" state of the corresponding 9 pixels driven at $30 \mathrm{~V}$ voltage.

Figure 2.4 shows the optical response of the devices measured by the colorimeter. The display devices were driven by applying a rectangular electrical waveform generated by a waveform generator and an amplifier, which was $30 \mathrm{~V} \mathrm{DC}$ at a duty cycle of $50 \%$ and frequency of $5 \mathrm{~Hz}$. For the devices with Teflon AF1600 and Hyflon AD60 as the hydrophobic insulator, the oil in the pixels was "open" when a $30 \mathrm{~V}$ voltage was applied onto the devices; and the oil could "close" completely when the electrical field was turned off. Both the "on" (pixel "open") and "off" (pixel "close") time was $<10$ ms (Figures 2.4a and 2.4b). For the EWD using Cytop 809A as the hydrophobic insulator, the oil film in pixels could "open" under the same conditions; however, the "close" was incomplete with a small area of oil film "open" in most pixels (Figure 2.4c). The "close" process took about $25 \mathrm{~ms}$ which was 2.5 times longer than the other two devices. 

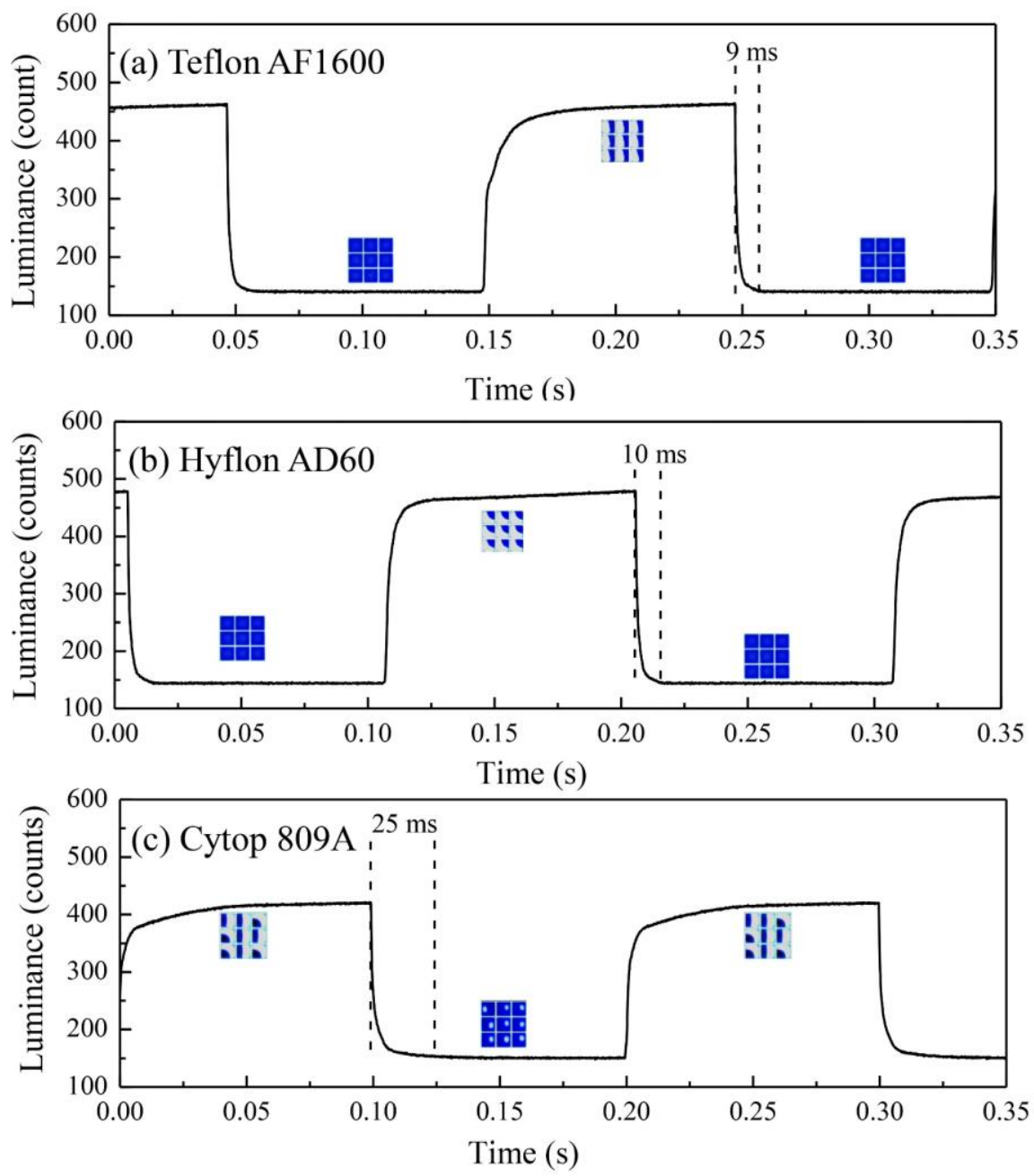

Figure 2.4 Optical response of the electrowetting displays using (a) Teflon AF1600, (b) Hyflon AD60 and (c) Cytop 809A as hydrophobic insulating layer. Inset: pictures of the "off" and "on" states of pixels in each sample.

From the experimental results, both the switching states and speed could be influenced by the surface wettability. To understand the mechanism behind, the force balance at the contact line was drawn in Figure 2.5. The interfacial tensions of oil/water, FP/oil and $\mathrm{FP} /$ water are presented as $\delta_{\mathrm{OW}}, \delta_{\mathrm{FO}}$ and $\delta_{\mathrm{FW}}$, respectively. The static force balance 
at the contact line is shown in Figure 2.5a. The steady contact angle $\theta_{\mathrm{Y}}$ is calculated by equation:

$$
\cos \theta_{Y}=\frac{\delta_{F O}-\delta_{F W}}{\delta_{O W}}
$$

(a)

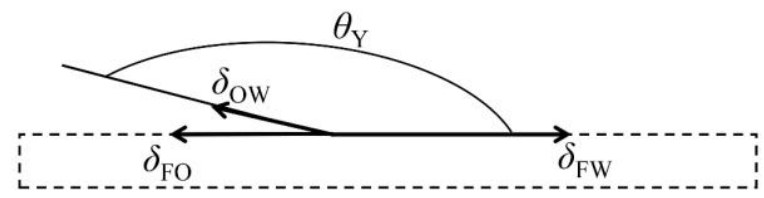

(b)

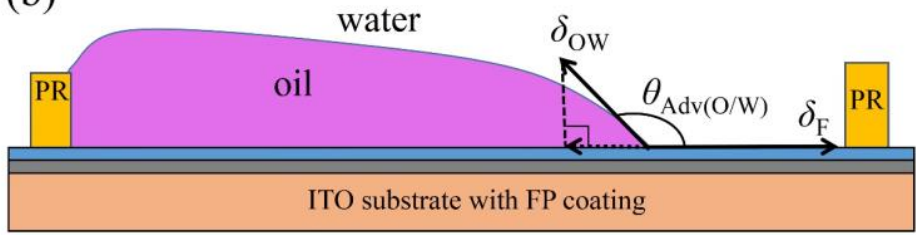

Figure 2.5 Schematic drawings of (a) the static force balance at the contact line and (b) the forces at the oil/water/FP contact line at a certain contact angle when oil film is spreading back to "off" state.

Since $\delta_{\text {ow }}$ is the same in all devices with different FPs $\left(\delta_{\mathrm{OW}}=19 \mathrm{mN} / \mathrm{m}\right.$ in this work), the larger oil/water contact angle on Cytop $809 \mathrm{~A}\left(\theta_{\mathrm{Y}(\mathrm{Cytop})}\right)$ results in smaller horizontal surface tension difference $\Delta \delta_{\mathrm{F}}=\delta_{\mathrm{FW}}-\delta_{\mathrm{FO}}$. According to Equation 2.2, the $\Delta \delta_{\mathrm{F}}$ of Teflon AF1600, Hyflon AD60 and Cytop 809A was $~ 18.9 \mathrm{mN} / \mathrm{m}, \sim 18.9 \mathrm{mN} / \mathrm{m}$ and 13.4 $\mathrm{mN} / \mathrm{m}$, respectively. Figure $2.5 \mathrm{~b}$ shows the forces at the oil/water/FP contact point when the oil film was moving back and spreading on FP surface. In a certain point when the oil film was advancing and the water phase was receding, the oil advancing contact angle $\theta_{\mathrm{Adv}(\mathrm{O} / \mathrm{w})}$ was shown in Figure 2.5b. The resultant force in the horizontal direction $F_{\mathrm{H}}$ is the joint result of $\Delta \delta_{\mathrm{F}}$ and the horizontal component of $\delta_{\mathrm{ow}}$. For the same reason, the larger $\Delta \delta_{\mathrm{F}}$ results in larger $F_{\mathrm{H}}$. As $\Delta \delta_{\mathrm{F}(\mathrm{Cytop})}$ is smaller than $\Delta \delta_{\mathrm{F}(\mathrm{Teflon})}$ and $\Delta \delta_{\mathrm{F}(\mathrm{Hyflon})}$, the $F_{\mathrm{H}(\mathrm{Cytop})}$ is correspondingly smaller, and the relatively low force in the horizontal direction leads to a low oil spreading speed, namely "off” speed. 
2.3.2 Effect of reflow temperature on EWD performance

Fluoropolymer surface repels to most fluids according to the low surface energy groups presented on the surface; therefore, reactive ion etching was applied to treat the FP surface to be hydrophilic for pixel layer coating. After lithography process, a thermal "reflow" process was applied to restore the FP surface to be hydrophobic in order to carry on electrowetting performance. The ITO-glass with pixels and FP layers were put into an oven and annealed for 2 hours under a certain temperature. During the "reflow" process, the low surface energy components in the FP film moved from the "bulk" to the surface since air presented at the interface. This resulted in fresh hydrophobic groups on the fluoropolymer surface ${ }^{[4]}$. Typically, the "reflow" temperature $\left(T_{\mathrm{re}}\right)$ needs to be high enough to allow most of the oxidized molecules to move into the bulk, so that the FP surface could turn back to be hydrophobic enough to contribute to the fast and reversible switch in EWD device. At the same time, the heat resistance of both FP and photoresist has to be considered. Commonly, $T_{\mathrm{re}}$ was set to be higher than $T_{\mathrm{g}}$ of the FPs but lower than $T_{\mathrm{g}}$ of the photoresist in order to get practical EWD devices.

To understand the influence of "reflow" process on the electrowetting performance of FP surface, both $\theta_{\operatorname{Rec}(\mathrm{W} / \mathrm{A})}$ and $\theta_{(\mathrm{O} / \mathrm{W})}$ were measured on the FP surfaces by varying $T_{\mathrm{r}}$, as shown in Figure 2.6. Before "reflow", $\theta_{\operatorname{Rec}(\mathrm{W} / \mathrm{A})}$ was 57,58 and $40^{\circ}$ for Teflon AF1600, Hyflon AD60 and Cytop 809A, respectively. With the increase of $T_{\mathrm{re}}$, $\theta_{\operatorname{Rec}(\mathrm{W} / \mathrm{A})}$ increased at the beginning and reach a plateau when $T_{\mathrm{re}}$ was 200,140 and $140{ }^{\circ} \mathrm{C}$ for Teflon AF1600, Hyflon AD60 and Cytop 809A, respectively. Cytop 809A showed lower $\theta_{\operatorname{Rec}(\mathrm{W} / \mathrm{A})}$ at both before and after "reflow" processes. However, $\Delta \theta_{(\mathrm{W} / \mathrm{A})}$ for the three FPs was similar, which were $53-62^{\circ}$. As seen from Figure $2.6 \mathrm{~b}, \theta_{(\mathrm{O} / \mathrm{w})}$ also increased with $T_{\text {re }}$, and reach the turning point at $T_{\text {re }}$ of 230,180 and $180{ }^{\circ} \mathrm{C}$ for Teflon AF1600, Hyflon AD60 and Cytop 809A, respectively. However, the $\theta_{(\mathrm{O} / \mathrm{w})}$ before "reflow" was all about $90^{\circ}$ for all three FPs after activation, and the $\theta_{(\mathrm{O} / \mathrm{w})}$ at the plateau was $\sim 10^{\circ}$ for Teflon AF1600, $\sim 10^{\circ}$ for Hyflon AD60, and $\sim 45^{\circ}$ for Cytop $809 \mathrm{~A}$. This means that $\Delta \theta_{(\mathrm{O} / \mathrm{w})}$ was $\sim 80^{\circ}$ for Teflon Teflon AF1600, $\sim 80^{\circ}$ for Hyflon AD60, and $\sim 45^{\circ}$ for Cytop 809A.

EWD devices have been prepared using the three FPs as hydrophobic insulating layers and "reflow" at various temperatures. Qualified electrowetting performance was observed when Hyflon AD60 and Cytop 809A was reflowed at $180{ }^{\circ} \mathrm{C}$; and no obvious difference was found when $T_{\mathrm{re}}$ was 200 and $230{ }^{\circ} \mathrm{C}$. For EWD devices with 
Teflon AF1600 as the hydrophobic insulating layer, $T_{\mathrm{re}} \geq 230{ }^{\circ} \mathrm{C}$ was required to achieve a reversible and fast pixel switching. This confirms that the reflow temperature is very important for restoring the surface wettability of different FPs and resulted EWD performance.
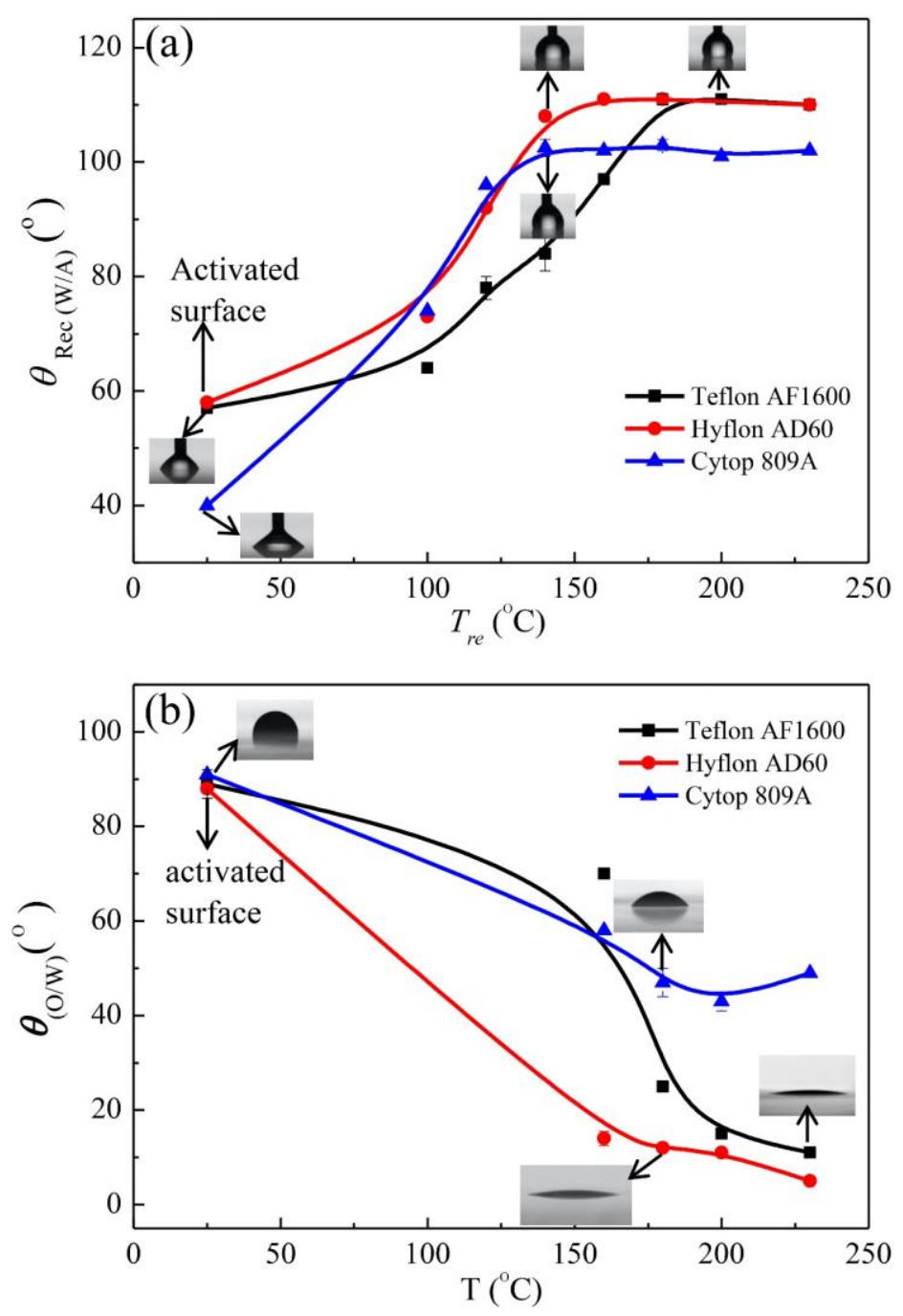

Figure 2.6 (a) $\theta_{(\mathrm{W} / \mathrm{A})}$ and and (b) $\theta_{(\mathrm{O} / \mathrm{w})}$ varying with "reflow" temperature on Teflon AF1600, Hyflon AD60 and Cytop 809A surfaces. 
On one hand, "reflow" at high temperature could achieve better recovery of the FP surfaces and EWD performance; however, higher temperature may bring failure issues to the EWD devices. As shown in Figure 2.7, obvious bending and dislocation was observed at the edge of the devices after "reflow" according to the difference of $T_{\mathrm{g}}$ and coefficient of expansion between FPs and pixel wall materials. Figures 2.7(af) show the edges of the devices annealed at 180 and $230{ }^{\circ} \mathrm{C}$. The pixel walls stayed on the FP surfaces without obvious bending and dislocation for the devices reflowed at $180{ }^{\circ} \mathrm{C}$; however, obvious edge bending and large dislocation was found when reflowed at $230{ }^{\circ} \mathrm{C}$. The largest shift between Teflon AF1600 and pixels was $120 \mu \mathrm{m}$, which occupied 4/5 length of a pixel (Figure 2.7d). Regarding to the devices reflowed at $230{ }^{\circ} \mathrm{C}$ using Hyflon AD60 and Cytop 809A, the FP layers at edge area were destroyed. The Hyflon AD60 material in the pixels at the edge area became very thin (observed by microscope) and even gathered at the pixel corners and accumulated to a bulge with thickness of $>13 \mu \mathrm{m}$, as shown in Figure 2.7e. The Cytop 809A film was partially torn at the edge, as shown in Figure 2.7f.

The EWD devices performance was also carried out to verify these findings. The EWD device with Teflon AF1600 reflowed at $180{ }^{\circ} \mathrm{C}$ could not realize completely reversible switching. The oil films in the pixels could not close completely after being switched off, which was consistence with previous findings. This might be caused by the less hydrophobicity of the surface. The electrodes in the edge area of devices with Hyflon AD60 and Cytop 809A reflowed at $230{ }^{\circ} \mathrm{C}$ were damaged immediately when voltages were applied. The EWD devices with hydrophobic insulating layers based on Teflon AF1600, Hyflon AD60 and Cytop 809A reflowed at temperature of 230, 180 and $180^{\circ} \mathrm{C}$, respectively, performed well. $50 \%$ duty cycle for 36000 "on"-"off" cycles $(2 \mathrm{~h}$ ) durable testing was achieved when driven by $30 \mathrm{~V}$ rectangular wave with $5 \mathrm{~Hz}$ frequency, as shown in Figures 2.7(g-i). 


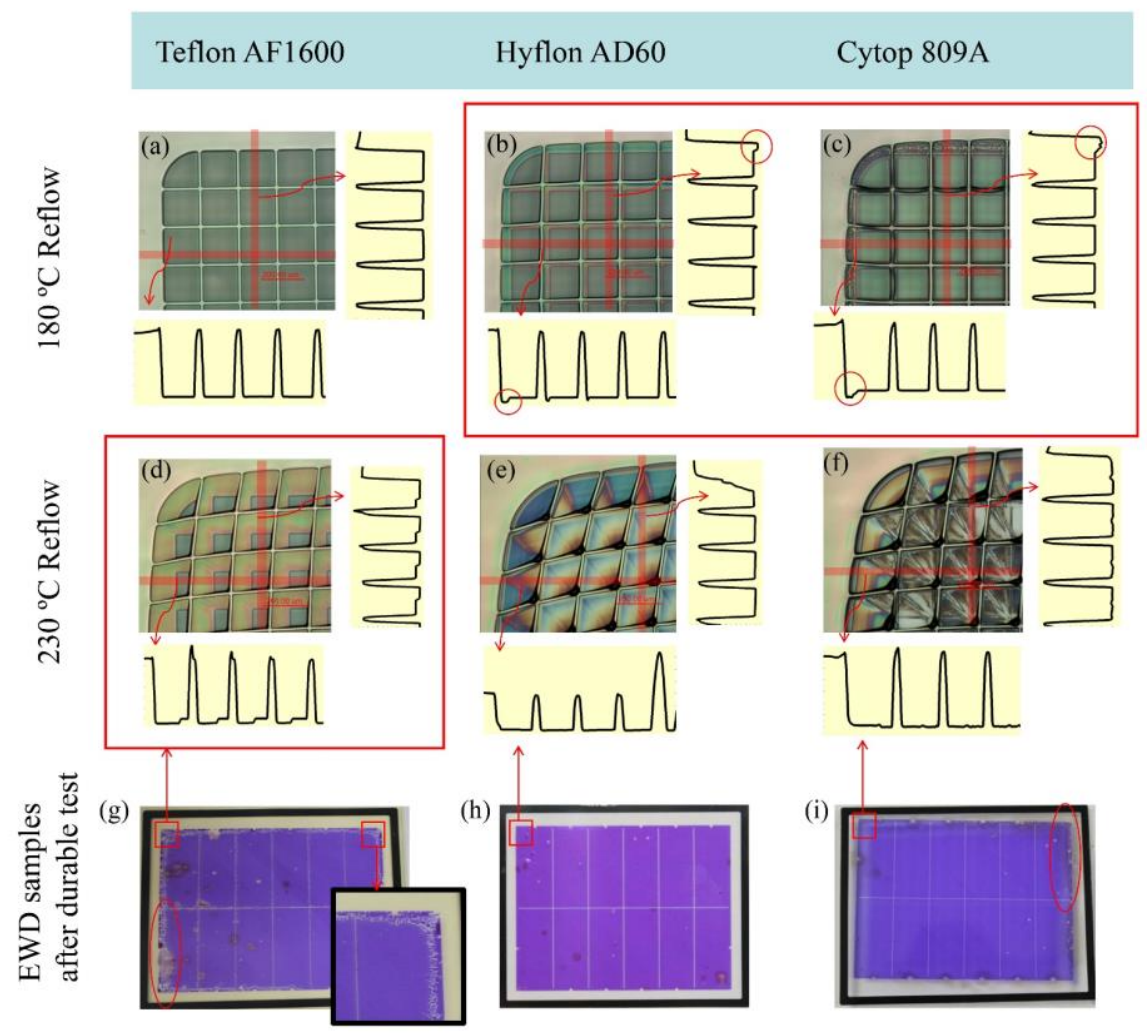

Figure 2.7 Optical microscope images and surface profile measurements of pixels and FPs based on Teflon AF1600 (a), Hyflon AD60 (b), and Cytop 809A (c) reflowed at $180{ }^{\circ} \mathrm{C}$; and Teflon AF1600, (d), Hyflon AD60 (e), and Cytop 809A (f) reflowed at $230{ }^{\circ} \mathrm{C}$. The red lines were the surface profiler measurement area. Pictures of the sample devices after being driven by $30 \mathrm{~V}$ rectangular waveform with $5 \mathrm{~Hz}$ frequency and $50 \%$ duty cycle for 36000 "on"-"off" cycles (2 h) durable testing with Teflon AF1600 (g), Hyflon AD60 (h), and Cytop 809A (i) reflowed at 230,180 , and $180^{\circ} \mathrm{C}$, respectively, as the hydrophobic layer. Inset of $(\mathrm{g})$ is the result of the colored oil "overflow" and jumping to the adjacent pixels.

To understand the reflow caused failures, the interfaces among pixel walls and FPs were measured using a scanning electron microscopy (SEM), as shown in Figure 2.8. When Teflon AF1600 and the pixel wall materials were annealed at $230{ }^{\circ} \mathrm{C}$, the pixel wall and Teflon AF1600 layer stayed close; however, Teflon AF1600 climbed up on the pixel wall (Figure 2.8a). Cracks of the Teflon AF1600 layer were also found, which may induce the breakdown failure in the devices. In Figure 2.8b, little FP 
climbing to the pixel walls was found; however, pixel wall delamination and FP depletion was observed. This might because of the low $T_{\mathrm{g}}$ of Hyflon AD60 $\left(120^{\circ} \mathrm{C}\right)$. As seen from Figure 2.8c, the Cytop 809A climbed up to the pixel wall and adhered tightly to the ITO surface. This might also explain the tearing patterns observed in Figure 2.7f. On the other hand, such a combination could result in less defects or pinholes in the central region of the display devices.

(a)

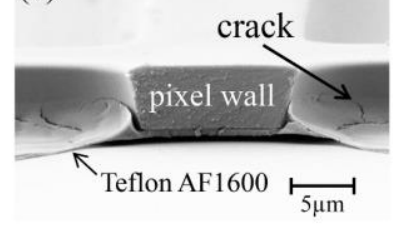

(b)

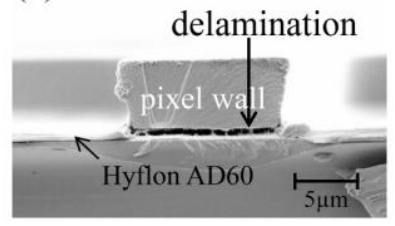

(c)

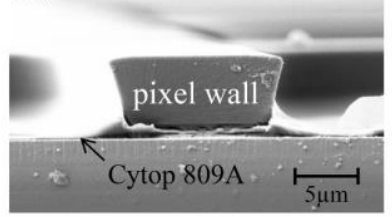

Figure 2.8 SEM images of the cross-sectional view of the FP-pixel interfaces: (a) Teflon AF1600 reflowed at $230{ }^{\circ} \mathrm{C}$, (b) Hyflon AD60 reflowed at $180{ }^{\circ} \mathrm{C}$, and (c) Cytop 809A reflowed at $180^{\circ} \mathrm{C}$.

\subsection{Conclusion}

Fluoropolymers have been chosen as the hydrophobic insulating layer to achieve electrowetting performance. In this work, three FPs based on Teflon AF1600, Hyflon AD60 and Cytop 809A have been evaluated for their applications in electrowetting displays. Reversible and fast optical switch could be realized in EWD devices with Teflon AF1600, Hyflon AD60 or Cytop 809A as the insulating and hydrophobic layers. The oil-in-water contact angle $\left(\theta_{(\mathrm{O} / \mathrm{w})}\right)$ was introduced as a more reliable way to evaluate the surface wettability of the FPs in EWDs. The $\theta_{(\mathrm{O} / \mathrm{w})}$ on Teflon AF1600 and Hyflon AD60 surfaces were both $<10^{\circ}$, resulting in fast pixel switch with "open" and "close" time of $<10 \mathrm{~ms}$. The Cytop 809A showed less hydrophobicity compared to Teflon AF1600 and Hyflon AD60 according to the dominance of $-\mathrm{CF}_{2}$ - groups. $\theta_{\text {(O/w) }}$ on Cytop 809A surface was $\sim 45^{\circ}$, leading to slower and incomplete "close" of pixels. Moreover, the "reflow" temperature was found to significantly affect the performance of EWD devices. Low $T_{\text {re }}$ would cause less recovery of FP surfaces, reducing the reversibility and completeness of pixel switching. However, a higher $T_{\text {re }}$ would cause the dislocation or crack of the FPs, inducing the breakdown failure of the EWD device. The optimum $T_{\text {re }}$ was found to be 230,180 and $180{ }^{\circ} \mathrm{C}$ for the films 
based on Teflon AF1600, Hyflon AD60 and Cytop 809A, respectively, to achieve a reliable EWD device. In summary, the slight wettability difference of FPs could induce big variation in EWD performance; and the "reflow" temperature plays a key role for EWD fabrication and performance. These results may help us deeply understand the electrowetting phenomenon and find ways to improve quality and durability of electrowetting-based devices in the future.

\section{Acknowledgments}

The authors appreciate Yingying Dou and Guimei Qin for lithography process, Yuanyuan Guo for device assembly, and Jieping Cao for contact angle measurements. The authors acknowledge the financial support from the National Key Research and Development Program of China (No. 2016YFB0401502), the National Natural Science Foundation of China (Nos. 61574065 and 51561135014), Guangdong Innovative Research Team Program (No. 2013C102), Guangdong Province Grant Nos. 2016B090906004, 2017B020240002, 2015B090913004, 2016B090918083, and Shenzhen Science and Technology Plan (No. GQYCZZ20150721150406). This work was also supported by Guangdong Provincial Key Laboratory of Optical Information Materials and Technology (No. 2017B030301007), Guangzhou Key Laboratory of Electronic Paper Displays Materials, Devices (201705030007), MOE International Laboratory of Optical Information Technologies, the 111 Project, and South China Normal University scholarship for oversea study.

\section{Contributions}

Hao Wu, Robert A. Hayes, and Guofu Zhou conceived and designed the experiments; Hao $\mathrm{Wu}$ performed the main experiments; Hao $\mathrm{Wu}$ and Lingling Shui analyzed the data and wrote the manuscript; Fahong Li, Alex Henzen, Lingling Shui and Guofu Zhou revised the manuscript.

\section{References}

[1] G. Lippmann, Gauthier-Villars Paris, France:, 1875.

[2] R. A. Hayes, B. J. Feenstra, Nature 2003, 425, 383.

[3] H. Wu, B. Tang, R. Hayes, Y. Dou, Y. Guo, H. Jiang, G. Zhou, Materials 2016, 9, 707. 
[4] D. Kim, A. Steckl, Langmuir 2010, 26, 9474.

[5] D. Witters, N. Vergauwe, S. Vermeir, F. Ceyssens, S. Liekens, R. Puers, J. Lammertyn, Lab on a Chip 2011, 11, 2790.

[6] S. Terrab, A. M. Watson, C. Roath, J. T. Gopinath, V. M. Bright, Optics express 2015, 23, 25838.

[7] T. Krupenkin, J. A. Taylor, Nature communications 2011, 2, 448.

[8] F. Mugele, J.-C. Baret, Journal of physics: condensed matter 2005, 17, R705.

[9] E. Seyrat, R. A. Hayes, Journal of Applied Physics 2001, 90, 1383.

[10] S. Berry, J. Kedzierski, B. Abedian, Journal of Colloid and Interface Science 2006, 303, 517.

[11] Y.-Y. Lin, R. D. Evans, E. Welch, B.-N. Hsu, A. C. Madison, R. B. Fair, Sensors and Actuators B: Chemical 2010, 150, 465.

[12] J. K. Lee, K.-W. Park, H.-R. Kim, S. H. Kong, Sensors and Actuators B: Chemical 2011, 160, 1593.

[13] B. Koo, C.-J. Kim, Journal of Micromechanics and Microengineering 2013, 23, 067002.

[14] H. You, A. Steckl, Applied physics letters 2010, 97, 023514.

[15] C. Hao, Y. Liu, X. Chen, Y. He, Q. Li, K. Li, Z. Wang, Scientific reports 2014, 4, 6846.

[16] E. Bormashenko, R. Pogreb, Y. Bormashenko, R. Grynyov, O. Gendelman, Applied Physics Letters 2014, 104, 171601.

[17] G. Beni, S. Hackwood, Applied Physics Letters 1981, 38, 207.

[18] J. Chen, J. Yang, Z. Li, X. Fan, Y. Zi, Q. Jing, H. Guo, Z. Wen, K. C. Pradel, S. Niu, ACS nano 2015, 9, 3324.

[19] X. Chen, T. He, H. Jiang, B. Wei, G. Chen, X. Fang, M. Jin, R. A. Hayes, G. Zhou, L. Shui, Displays 2015, 37, 79.

[20] K. Zhou, J. Heikenfeld, K. Dean, E. Howard, M. Johnson, Journal of Micromechanics and Microengineering 2009, 19, 065029.

[21] T. He, M. Jin, J. C. Eijkel, G. Zhou, L. Shui, Biomicrofluidics 2016, 10, 011908. 


\section{Supporting information:}

Table S1: Molecular structures and properties of the three selected fluoropolymers.

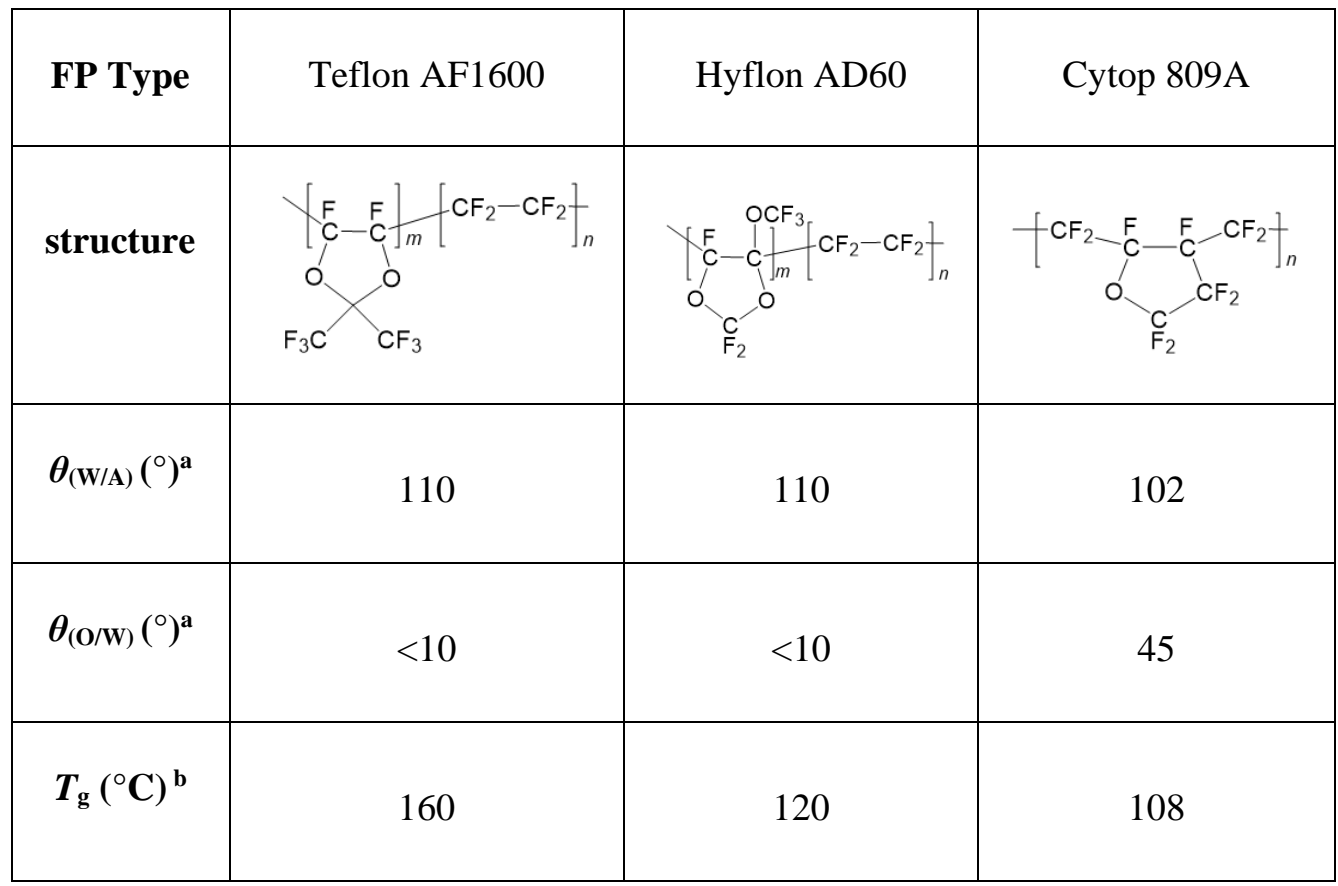

a, experimental value by averaging four measurements.

$\mathrm{b}$, data from the datasheet of commercial product manual. 


\section{Chapter 3}

\section{Large-area high-contrast hydrophobic/hydrophilic patterned surface for robust electrowetting devices*}

In Chapter 2, we demonstrated that the hydrophobicity of fluoropolymer is essential for electrowetting(EW) devices. However, building hydrophilic structures firmly on the hydrophobic coatings has been a challenge for EW device fabrication. In this chapter, we propose a reconstructive approach to keep hydrophobicity of Teflon AF surfaces and at the same time, enhance the hydrophilicity of patterned microstructures by local plasma etching method with a self-assembled protection mask. The extremely high wettability contrast with a large oil/water contact angle difference $\left(\Delta \theta_{\mathrm{o} / \mathrm{w}}\right)$ of $175^{\circ}$ is reached between the hydrophobic and hydrophilic surfaces. Electrowetting display devices are fabricated to verify the feasibility of the approach.

\footnotetext{
${ }^{*}$ This chapter is based on publication: H. Wu, L. Shui, F. Li, R. Hayes, A. Henzen, F. Mugele, G. Zhou, Large-Area High-Contrast Hydrophobic/Hydrophilic Patterned Surface for Robust Electrowetting Devices, ACS Applied Nano Materials, 2 (2019) 1018-1026.
} 


\subsection{Introduction}

Stenocara beetles in Namib Desert utilize the hydrophobic/hydrophilic patterned surfaces (HHPS) on their body to capture small water droplets ${ }^{[1-3]}$. HHPS can also control the direction of water droplet movement on the rice leaf ${ }^{[4]}$. Recently, HHPS have attracted a lot of attentions due to their fundamental interests and promising application perspectives ${ }^{[5-7]}$, including biomimetic studies for spatial controlling of condensation and freezing ${ }^{[8]}$, fog collections for solving global freshwater crisis ${ }^{[9-10]}$, biomedical devices for molecular sensing, targeted antibacterial and drug delivery ${ }^{[11-}$ ${ }^{12]}$, green electronic devices of light valves ${ }^{[13]}$, and electrowetting displays ${ }^{[14-15]}$. In most cases, the wettability contrast between the hydrophilic and hydrophobic areas is the key for achieving designed functional properties ${ }^{[16-17]}$. For instance, HHPSs are required in an electrowetting display device to achieve well confinement of the dualliquid system, with the non-polar oil assembled to the hydrophobic area (pixels) surrounded by hydrophilic walls and coexisting with aqueous solution ${ }^{[13,15]}$. The high contrast in wettability is challenging for micro- and nano-patterning technologies. Because of the low free energy of the hydrophobic coating (e.g. Teflon), it is difficult to directly pattern hydrophilic patterns onto a hydrophobic surface; and delamination happens easily according to the mismatch of thermal expansion between two layers when a high temperature process is applied ${ }^{[18-20]}$.

Amorphous fluoropolymers, are mostly used materials for hydrophobic coatings and can be fabricated by various methods, such as spin-coating, dip-coating and screen-printing ${ }^{[21-23]}$. Photoresists are usually patterned on the fluoropolymer surfaces via a lithography approach to form HHPS ${ }^{[20-23]}$. As the relatively hydrophilic photoresist is hardly compatible with the hydrophobic fluoropolymer surface, a regular way is to utilize reactive ion etching (RIE) to convert fluoropolymer surface to be hydrophilic, allowing photoresist layer to be coated and patterned ${ }^{[24-25]}$. Afterwards, a thermal reflow process is applied to recover the fluoropolymer's hydrophobicity to achieve a HHPS (Figure 3.1a) ${ }^{[15]}$. We denote the HHPS fabricated using this "RIE-reflow" methods as "RIE-reflow" HHPS (RR-HHPS). In this way, undesirable chemical and physical contaminations could be introduced to the fluoropolymer layer during this "RIE-reflow" process ${ }^{[26]}$. It was reported that unrecoverable damage of the fluoropolymer surface would decrease the surface hydrophobicity and dielectric strength, resulting in non-uniform onset voltage of electronic devices ${ }^{18,24,26}$. On the other hand, highly viscous photoresist can also be patterned on hydrophobic surfaces directly ${ }^{[27]}$; however, these structures can be easily peeled off or delaminated because of lacking of strong chemical bonds ${ }^{[24,27]}$. Zhang 
H. et al ${ }^{[18]}$ applied a hydrophilic $\mathrm{SiO}_{2}$ coating as a sandwiched layer between fluoropolymer and photoresist layers. Yet the E-beam deposition of $\mathrm{SiO}_{2}$ layer led to the degradation of hydrophobicity of fluoropolymer surface. To achieve a sound fabrication process for making hydrophobic/hydrophilic surfaces, researchers tend to employ approaches of either decreasing the wettability contrast of two layer coatings or via a surface modification and recovery process [18, 22, 28]. Both strategies compromise on the wettability contrast.

In this work, we propose and evaluate a reconstructive approach for large-area fabrication of highly hydrophilic patterns on a hydrophobic fluoropolymer coating, creating a reconstructive hydrophobic/hydrophilic patterned surface (denoted as RCHHPS) with high wettability contrast. By encapsulating "damaged" hydrophobic Teflon AF 1600 (AF) surface with a fresh one, both hydrophobicity and dielectric strength of the coatings were preserved as on a pristine AF surface; and the local RIE activation of the photoresist via a self-assembled mask resulted in super hydrophilic micro-patterns. Compared with the conventional RR-HHPS, the RC-HHPS presented in this work exhibits superior surface wettability contrast and dielectric strength. RCHHPS could be utilized in any application that requires HHPS. Particularly, RCHHPS itself is already a high-quality and robust electrowetting platform. We fabricate electrowetting devices based on this engineered substrate to demonstrate the beneficial effects derived from the RC-HHPS.

\subsection{Experimental Section}

\subsubsection{Materials and Equipment}

Teflon AF1600 (Dupont, Shanghai, China) dissolved in fluorinate electronic liquid (FC-43; Minnesota Mining \& Manufacturing Company, Saint Paul, USA) at concentrations of 3.0 and $3.7 \mathrm{wt} . \%$ was used as amorphous fluoropolymer material in this work. The hydrophilic micro-patterns were made of the negative photoresist of SU8-3005 (MicroChem Corp., Westborough, USA). Positive photoresist of SUN$120 \mathrm{P}$ was applied as the material for creating the protection mask, which was purchased from SUNTIFIC Company (Weifang, China). Indium tin oxide (ITO) coated glass with electrical resistance of $100 \Omega / \square$ was purchased from Guangdong Jimmy Glass Technology Ltd. (Foshan, China). The conductive liquid was $1.0 \mathrm{mM}$ $\mathrm{NaCl}$ solution with measured conductivity of $\sim 110 \mu \mathrm{S} / \mathrm{cm}$. The colored oil was prepared by dissolving the synthesized dyes in Decane (Micklin, Shanghai, China) ${ }^{[29]}$. 
The interfacial tension of colored oil ( $0.21 \mathrm{M}$ dye in decane solution)/conductive liquid (1.0 mM NaCl aqueous solution) was measured to be $19 \mathrm{mN} / \mathrm{m}$.

ITO coated glass sheets were cleaned by a commercial cleaning line (KJD-7072ST, KEJINGDA Ultrasonic Equipment Co., Ltd., Shenzhen, China) prior to use. Fluoropolymer solution was spin-coated on the surface of ITO glass using a spin coater (KW-5, Institute of Microelectronics Chinese Academy of Sciences, Beijing, China). Reactive Ion Etching (RIE) tool (ME-6A) was purchased from the Institute of Microelectronics Chinese Academy of Sciences (Beijing, China). Lithography process was performed by using an aligner (URE-2000/35, Institute of Optics and Electronics, Chinese Academy of Sciences, Chengdu, China). An oven (5FG-01B, Huangshi, China) was used for all thermal treatment processes. Protection layer was filled into the gaps between hydrophilic patterns by a dip coating equipment (SYDC, Shanghai SAN-YAN Technology Co., Ltd, China).

\subsubsection{Fabrication methods}

Hydrophobic AF film fabrication

Teflon AF 1600 (AF) films with thickness of $400 \pm 20$ and $800 \pm 20 \mathrm{~nm}$ were prepared by spin-coating 3.0 and $3.7 \mathrm{wt} . \%$ AF 1600 solutions, respectively, on ITO-glass surfaces. The substrate was cured on a hot plate at $85^{\circ} \mathrm{C}$ for $5 \mathrm{~min}$ and then in an oven at $185^{\circ} \mathrm{C}$ for $30 \mathrm{~min}$. Thus, a "virgin" or "fresh" $\mathrm{AF}$ film was obtained. Afterwards, RIE treatment was carried at $5 \mathrm{~W}$ power for $10 \mathrm{~s}$ to tune the $\mathrm{AF}$ surface to become a hydrophilic "RIE AF" film. A thermal annealing process at $230{ }^{\circ} \mathrm{C}$ for $2 \mathrm{~h}$ in an oven was then applied to recover the hydrophobicity of the AF surface. Such an AF film at this stage was denoted as a "RIE-reflow AF" film.

\section{Conventional "RIE-reflow" method for HHPS preparation}

The conventional "RIE-reflow" fabrication methods for HHPS (RR-HHPS) is shown in Figure 3.1a. In this process, amorphous fluoropolymer (Teflon AF1600) was coated as an insulator and hydrophobic layer by the method describe in the previous session of 2.2.1. Considering the low surface tension of an AF coating, to allow hydrophilic patterns to be fabricated onto it, a RIE process with oxygen plasma powered at $5 \mathrm{~W}$ for $10 \mathrm{~s}$ was applied to enhance the surface roughness and wettability of AF film. After fabricating the hydrophilic patterns (SU8-3005) by a lithography process, a 
thermal reflow process at high temperature of $230{ }^{\circ} \mathrm{C}$ was employed to recover the hydrophobicity of the AF surface.

\section{Proposed "Reconstruction" method for HHPS preparation}

In this work, we propose a surface "non-damaging" approach for fabricating HHPS with both highly hydrophilic surfaces and virgin hydrophobic fluoropolymer surfaces. The process is illustrated in Figure 3.1b, which is denoted as a "reconstruction" process. A layer of $\sim 400 \mathrm{~nm}$ Teflon AF 1600 (AF) was coated on the ITO-glass as a hydrophobic insulating layer. The relatively hydrophilic upper surfaces of the micro walls were made by SU8-3005 (SU8) via a photolithography process on the RIE treated AF surface. Afterwards, a layer of $\sim 400 \mathrm{~nm}$ fresh AF was spin coated (at spin rate of $1500 \mathrm{rpm}$, AF concentration of $3.7 \mathrm{wt} . \%$ ) on the substrate covering both activated AF and SU-8 surfaces. According to the low surface energy of AF material, the freshly coated AF layer could spread and cover the patterned substrate to form a continuous coating layer. In addition, given the selected solvent of FC-43 with a very high boiling point of $174^{\circ} \mathrm{C}$, the Teflon AF solution tends to spread on the surface and level itself to a large extent before the solvent completely evaporates. As a result, a relatively homogeneous film is obtained. Although the coating may not be completely conformal with local inhomogeneity existing around the corners (SEM picture shown in Figure 3.1), there is a complete AF layer underneath SU8 and the fresh AF layer; the problems, such as leakage, derived from the incomplete conformal issue could be avoided mostly. Afterwards, a protection layer (positive photoresist of SUN-120P) was filled into the gaps between pixel walls via a dip coating process at a falling and pulling rate of $17 \mathrm{~mm} / \mathrm{s}$. The second RIE process (power of $200 \mathrm{~W}$ for 80 s) was applied to etch away the exposed AF layer on the top of SU8 wall surfaces. After cleaning up the protection layer by $\mathrm{NaOH}$ solution $(8.0 \mathrm{wt} \%)$ following with a thorough water cleaning, the Reconstructive HHPS (RC-HHPS) substrate with new hydrophobic (fresh AF) surfaces in the wells and firmly hydrophilic (activated SU8) upper wall surfaces, was obtained. As the materials of the top layer and the bottom layer are identical, the excellent adhesion between these two layers allows the micro patterned Teflon AF sticking perfectly on the bottom layer without delamination. 


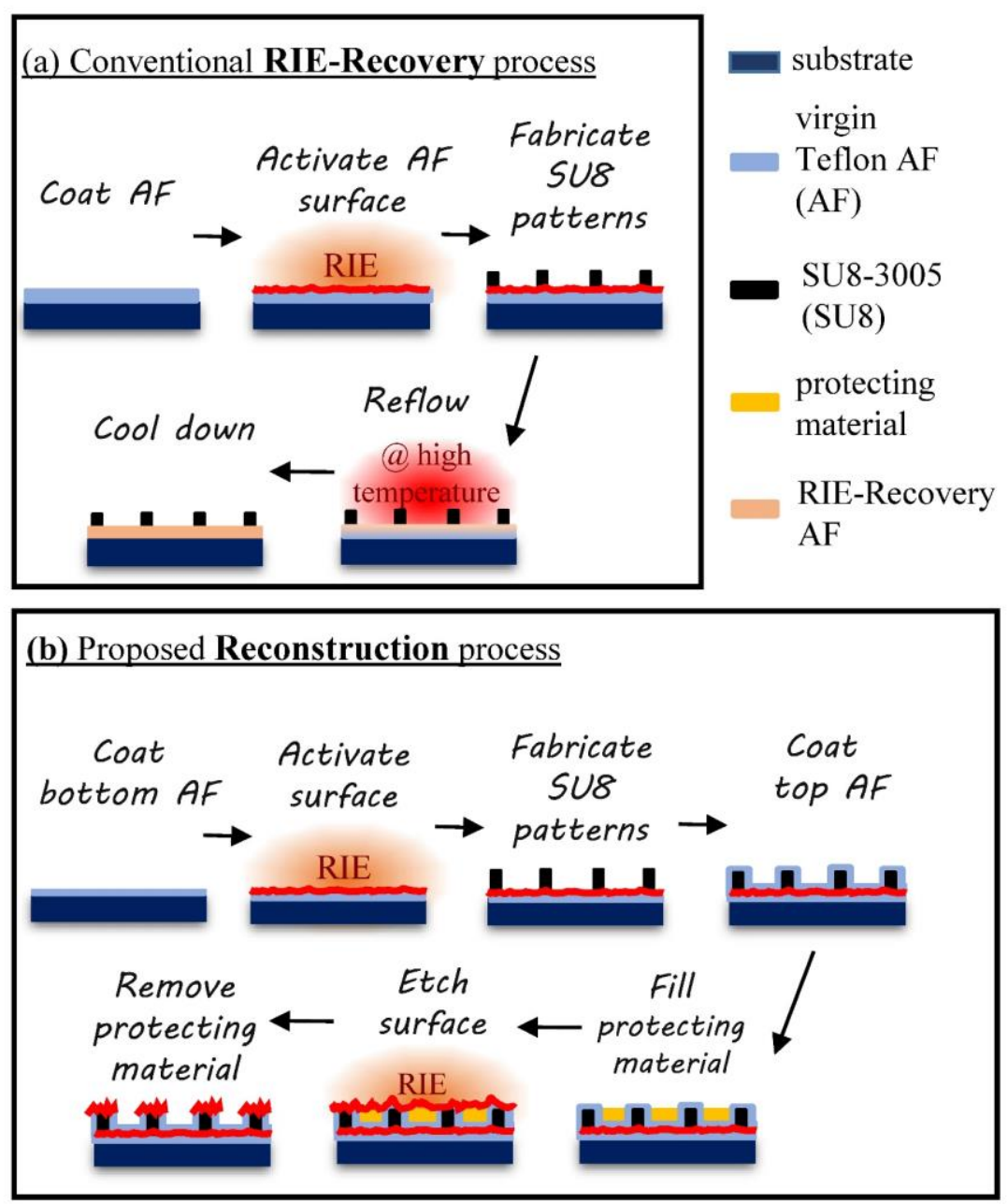

Figure 3.1. Schematic drawing of (a) the conventional RIE-reflow process and (b) the proposed reconstruction process for fabricating hydrophobic/hydrophilic patterns. Inset of (b) is a cross-sectional image detected by a scanning electron microscope (SEM).

\subsubsection{Characterization}

Thickness of the fluoropolymer film was measured by a stylus profiler (Dektak XT, BRUKER, Germany). Oil/water contact angle on the surface of films was measured 
by a contact angle goniometer (POWEREACH, Shanghai Zhongchen Digital Technology Apparatus Co., Ltd. Shanghai, China). Atomic force microscopy (AFM) measurements were carried out using a MultiMode8 (Bruker, Guangzhou, China) with a monocrystalline cantilever of Bruker ScanAsyst. Instantaneous current profiles were recorded using a pico-ammeter (Keithley 6487, USA) equipped with a platinum coated needle. A $10 \mu \mathrm{L} 1 \mathrm{mM} \mathrm{NaCl}$ aqueous droplet on the film surface was applied as the top electrode. The capacitance of the fluoropolymer film was measured by an impedance analyser (6500B, WAYNE KERR, UK). An optical microscope (CTX41, Olympus, Tokyo, Japan) equipped with a high-speed camera (Phantom MiRO M110, Wayne, USA) was used to visualize and record the fluid movement in devices. A scanning electron microscope (SEM) (ZEISS Ultra 55, Carl Zeiss, Jena, Germany) was used to observe the sample structures. The opening ratio of pixels in the electrowetting display devices was calculated by analyzing the captured images using MATLAB program.

\subsection{Results and discussion}

\subsubsection{Topology and surface wettability of RR-HHPS and RC-HHPS}

The top-view schematic diagrams of array-patterned RR-HHPS and RC-HHPS are shown in Figures 3.2a and 3.2b, respectively. The surface pattern design for the RRHHPS and RC-HHPS are the same. The square hydrophilic patterns are $15 \mu \mathrm{m}$ wide (line) with an interval distance (pitch) of $150 \mu \mathrm{m}$ between the mid-line of the hydrophilic patterns. The cross-sectional schematic and scanning electron microscope (SEM) observation of RR-HHPS and RC-HHPS are presented in Figures 3.2(c-f). During the fabrication of RR-HHPS, a high temperature $\left(230{ }^{\circ} \mathrm{C}\right)$ above the glass transition $\left(T_{\mathrm{g}}\right)$ of AF was applied to recover the hydrophobicity of the AF surface which was damaged by the previous RIE process. We found that this high temperature led to a transformation of the AF film connecting the hydrophilic patterns. As the surface free energy of AF is $16.4 \pm 1.4 \mathrm{mN} / \mathrm{m}^{[30]}$ which is much lower than that of SU8 of $45.20 \pm 0.88 \mathrm{mN} / \mathrm{m}^{[31]}$, AF in the RR-HHPS climbs up the hydrophilic walls during the reflow process. This transformation may lead to unexpected issues of defects, delamination or ruptures ${ }^{[15]}$ in practical applications. In the RC-HHPS samples, some nano-sized particles appeared near the SU8 surfaces, which were caused by the heavy etching process. 


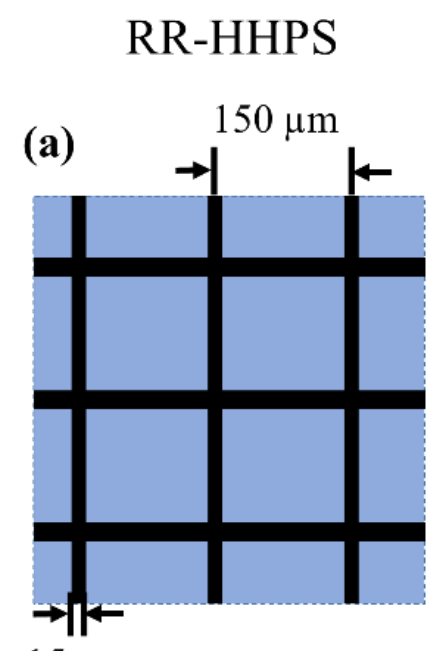

$15 \mu \mathrm{m}$

(c)

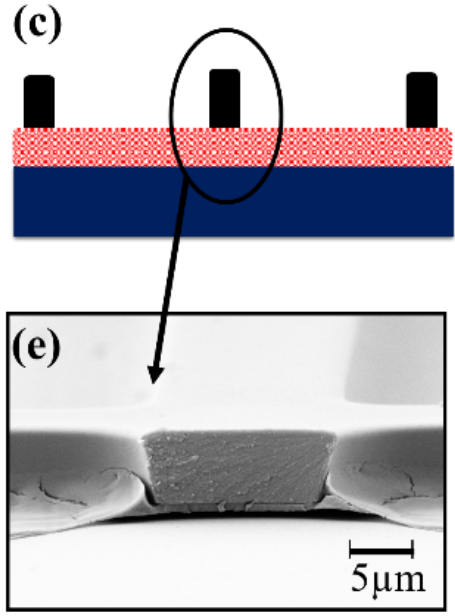

\section{RC-HHPS}

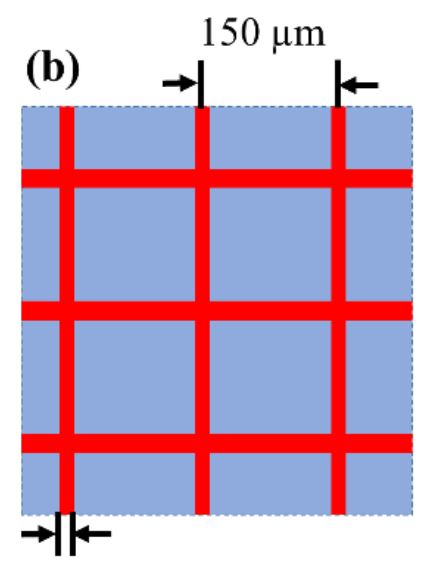

$15 \mu \mathrm{m}$

(d)

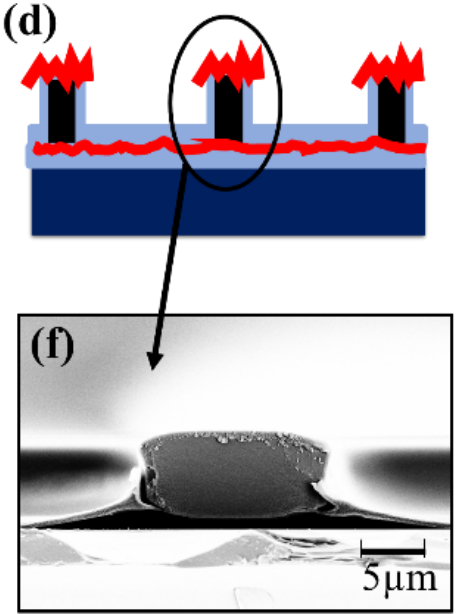

Figure 3.2 Schematic of the top view of (a) RR-HHPS and (b) RC-HHPS, and cross-sectional view of (c) RR-HHPS and (d) RC-HHPS. Scanning electron microscope (SEM) images of the cross-sectional observations of (e) a RR-HHPS and (f) a RC-HHPS. The height of the hydrophilic walls is about 6 and $7 \mu \mathrm{m}$ for RR-HHPS and RC-HHPS samples, respectively.

The wettability contrast of RR-HHPS and RC-HHPS is presented in Figure 3.3. Figures $3.3 \mathrm{a}$ and $3.3 \mathrm{~b}$ show the water contact angle in air $\left(\theta_{\mathrm{w} / \mathrm{a}}\right)$ and the oil contact angle in water $\left(\theta_{\mathrm{o} / \mathrm{w}}\right)$, respectively, on the surfaces. The interfacial tension of dyed oil/conductive water was $19 \mathrm{mN} / \mathrm{m}$. The insets are the captured profiles of the contact angle measurements. The wettability of the hydrophobic surfaces for RR-HHPS and 
RC-HHPS are similar. $\theta_{\mathrm{w} / \mathrm{a}}$ of the hydrophobic surfaces of RR-HHPS and RC-HHPS are both about $115^{\circ}$. The contact angle hysteresis of both surfaces are small, and the difference between water/air advancing contact angle and receding contact angle of both samples are less than $10^{\circ}$. There is an obvious difference of $\theta_{\mathrm{o} / \mathrm{w}}$ between the hydrophobic surfaces of the two samples, which is $10^{\circ}$ for RR-HHPS and $<5^{\circ}$ for RC-HHPS. This indicates that, during the conventional "RIE-reflow" process for HHPS, the reflow process at high temperature could recover the hydrophobicity of an AF surface to a large extent, yet there is still slight wettability difference in $\theta_{\mathrm{o} / \mathrm{w}}$, influencing the performance of a water-oil two phase system. On the contrast, the wettability difference between hydrophilic regions for RR-HHPS and RC-HHPS are pronounced. $\theta_{\mathrm{w} / \mathrm{a}}$ of hydrophilic areas of RR-HHPS and RC- HHPS are 60 and $10^{\circ}$, respectively. The advancing $\theta_{\mathrm{o} / \mathrm{w}}$ on RR-HHPS and RC- HHPS are 70 and $180^{\circ}$, respectively. As the oil droplet poorly wets the hydrophilic surfaces of RC- HHPS, there always exists a water layer on the hydrophilic surface and we had to use a syringe needle to hold the oil droplet to show the contact angle profile. For comparison, the $\theta_{\mathrm{o} / \mathrm{w}}$ data for all hydrophilic surfaces of RR-HHPS were measured in the same way. According to the contact angle hysteresis of hydrophilic surfaces of RR-HHPS, the advancing and receding $\theta_{\mathrm{w} / \mathrm{a}}$ are 69 and $55^{\circ}$, respectively; and the advancing and receding $\theta_{\mathrm{o} / \mathrm{w}}$ are 70 and $20^{\circ}$, respectively. The significant enhancement of hydrophilicity of the SU8 surfaces in RC- HHPS was caused by the heavy oxygen reactive ion etching (RIE) process. By treating the SU8 surfaces with oxygen plaster, the absolute amount of oxygen on the surfaces increases, while the amount of carbon decreases. The reported analysis of the XPS spectra indicates the formation of different oxygen species in a mixture of ether, aldehyde and carboxylic acid functional groups ${ }^{[31]}$. At the same time, physical roughness was introduced on the SU8 top surfaces due to the heavy RIE process. The hydrophilicity of the SU8 surface is thus highly increased because of the combination of chemical compounds and physical nano-roughness. As the hydrophobic AF regions in RC-HHPS are covered by the protection layer (a self-assembled mask produced by dip-coating method), its hydrophobicity was preserved. As a consequence, the contrast of $\theta_{\mathrm{o} / \mathrm{w}}$ on RC-HHPS is as high as $175^{\circ}$, which is significantly higher than that on the RR-HHPS of $60^{\circ}$. The contrast of $\theta_{\mathrm{w} / \mathrm{a}}$ on RC-HHPS is $105^{\circ}$, which is also much higher than that on the RRHHPS of $55^{\circ}$. The wettability contrast between the hydrophilic and hydrophobic regions is significantly enhanced by this reconstructive process. 

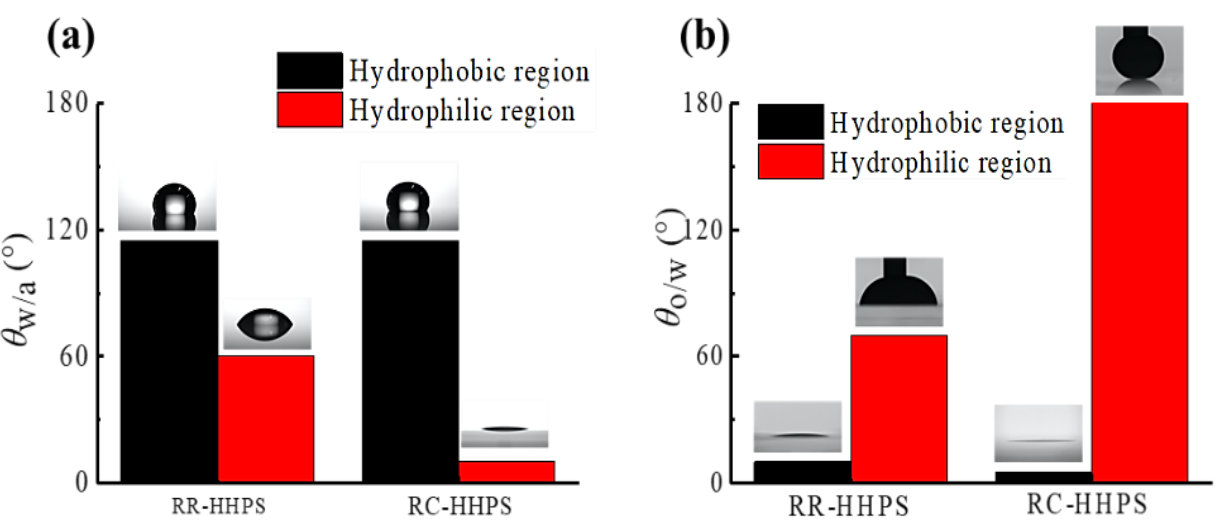

Figure 3.3 Comparison of (a) $\theta_{\mathrm{w} / \mathrm{a}}$ and (b) $\theta_{\mathrm{o} / \mathrm{w}}$ on the hydrophobic and hydrophilic region on RR-HHPS and RC-HHPS.

3.3.2 Morphology and electronic properties of "RIE-reflow" (RR) AF film and "Reconstructive"(RC) AF film.

The difference in wettability of the AF films fabricated by "RIE-reflow" (RR) and "Reconstructive"(RC) processes suggests that the reflow process can not completely recover the AF surface to the initial state. We investigated the influence of the RIE and reflow processes on AF surfaces by using an atomic force microscopy (AFM). The surface morphology (surface roughness) of the virgin AF, RIE AF (AF surfaces treated by RIE process) and RIE-reflowed AF (AF surfaces treated by RIE and thermal recovery processes) are shown in Figures 3.4 (a-c). The average roughness of the virgin $\mathrm{AF}, \mathrm{RIE} \mathrm{AF}$ and RIE-reflow $\mathrm{AF}$ surfaces are different, with the $R_{\mathrm{q}}$ (Root mean square average of height deviations) of $0.42,0.52$ and and $0.39 \mathrm{~nm}$, respectively. It seems that the RIE process makes the surface roughness increase; however, after the reflow process, the surface rough returns to original value. Thus, the overall roughness of virgin AF and RIE-reflow AF surfaces is similar. However, if we look at the Surface Area Difference (SAD: representing the percentage increase of the three-dimensional surface area over the two-dimensional surface area) ${ }^{[32]}$ of the surface height data, obvious difference among the three samples was observed. SADs are $0.20,0.61$ and $0.61 \%$ for the virgin, RIE and RIE-reflowed AF surfaces, respectively. Combining with the three dimensional features shown in Figures 3.4(ac), we found that the surface area was increased, and numerous nano-scaled islands 
were generated after RIE-reflow process. Overall, compared to the virgin AF surface, the RIE-reflow AF surface shows similar vertical roughness but higher specific surface area.

The adhesion force was also measured by using a Bruker ScanAsyst cantilever at a force of $1.3 \mathrm{nN}$. Figures 3.4(d-f) show the mean values of adhesion forces on virgin, RIE and RIE-reflow AF surfaces to be 3.22, 6.16 and $3.41 \mathrm{nN}$, respectively. The average adhesion value of the RIE-Reflowed AF surface $(3.41 \mathrm{nN})$ is similar to that of virgin AF surface $(3.22 \mathrm{nN})$. However, the SAD data of their adhesion forces increase dramatically after RIE treatment. SADs of the virgin, RIE and RIE-reflow AF surfaces were $0.46 \%, 9.32 \%$ and $9.99 \%$, respectively. Thus, the surface uniformity cannot be recovered after RIE treatment. Although it is widely believed that the thermal reflow process can recover the surface wettability of RIE treated AF surface by bringing oxidized molecules and other high energy groups toward the "bulk" of the film ${ }^{[5]}$, the difference in adhesion forces and their SAD indicate that there are still impurity phases on the AF surface after the high temperature thermal treatment. Therefore, such a thermal recovery process could not completely eliminate the RIE introduced physical and chemistry damages.

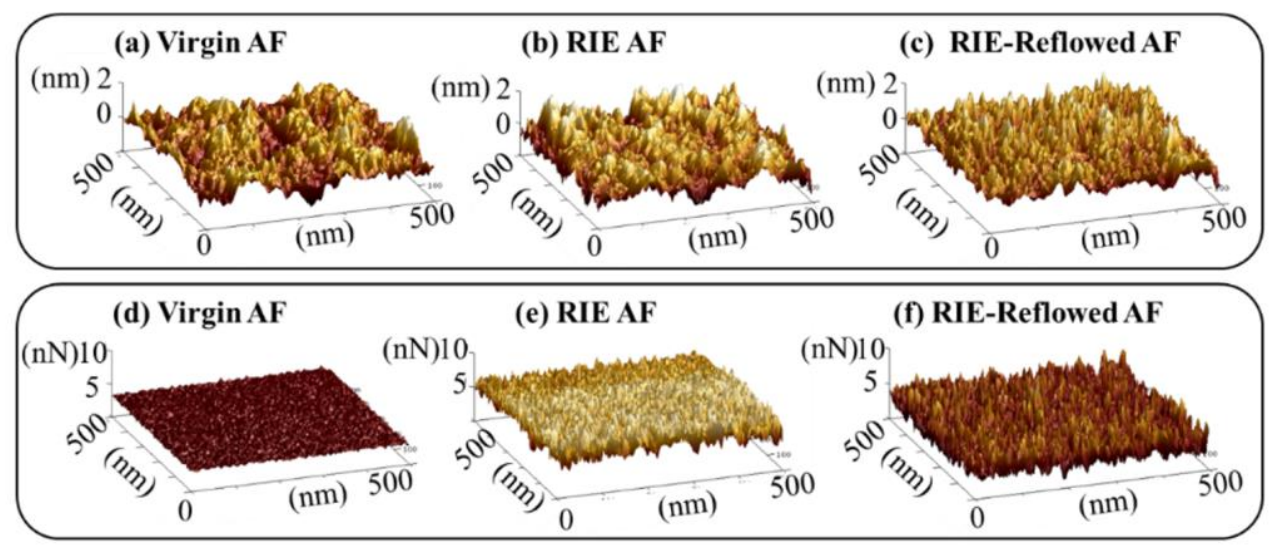

Figure 3.4. AFM measurements of the surface morphologies on (a) virgin (fresh) Teflon AF1600 (AF), (b) RIE-treated AF and (c) a RIE-reflowed AF surfaces; and surface adhesion forces on (d) fresh Teflon AF1600 (AF), (e) RIE treated AF surface, and (f) RIE-reflowed AF surfaces. All AF film thickness is $800 \pm 20 \mathrm{~nm}$. 
Except from the surface uniformity and hydrophobicity, the intact AF surface also benefits from its original dielectric strength for more reliable electrowetting performance. In conventional RIE-reflow process, the thermal recovery treatment may lead to the diffusion/immigration of RIE-induced high energy "impurities" from surface into bulk of the AF film ${ }^{[15]}$. Moreover, AF may also decompose at high temperature with small molecules being generated and oxygen components increased in the $\mathrm{AF}$ film ${ }^{[33]}$. Commonly, the impurities and oxides in dielectric films increase the leakage current ${ }^{[34-35]}$. Figure 3.5 shows the measured leakage currents of the virgin AF film, the RIE-reflow AF film, and the reconstructed AF film made by our proposed method, measured by a picoammeter with a platinum coated needle and $10 \mu \mathrm{L} \mathrm{NaCl}$ solution $(1 \mathrm{mM})$ as the top electrode.

The film thickness of the virgin, "RIE-reflow" and "Reconstruction" AF layers are all $\sim 800 \mathrm{~nm}$. RIE-reflow film was treated by RIE activation and thermal recovery at $230{ }^{\circ} \mathrm{C}$ for $2 \mathrm{~h}$. Reconstruction film is a bilayer AF film that comprises a $\sim 400 \mathrm{~nm}$ fresh AF layer stacked on a $\sim 400 \mathrm{~nm}$ layer of RIE treated AF surface. For comparison, the same RIE and photolithography parameters were employed in fabricating both RIE-reflow and Reconstruction films. Virgin and Reconstruction films show similar electric properties with low leakage current of $10^{-10} \mathrm{~A}$ at applied voltage of $U<120 \mathrm{~V}$ (Figure 3.5a). However, the leakage current of RIE-reflow film increases dramatically when $U$ is over $14 \mathrm{~V}$. This is detrimental for practical applications since the contact angle change is small $\left(<2^{\circ}\right)$ at such a low voltage $(U<14 \mathrm{~V})$. When $U$ increases to be $>14$ $\mathrm{V}$ to achieve a more pronounced contact angle change, $U=30 \mathrm{~V}$ for instance, the leakage current of RIE-reflow film is 3-5 orders of magnitude higher than that of virgin and Reconstruction films. These results suggest that the proposed "Reconstruction" strategy is highly reliable compared to conventional RIE-reflow process, showing similar electrical properties with virgin AF films.

Moreover, the water (10 $\mu \mathrm{L}$ of $1 \mathrm{mM} \mathrm{NaCl}$ solution) contact angles in air medium were measured on the three films (Figure 3.5b). The red dashed curve was calculated based on the Lippmann's equation ${ }^{15}, \cos \theta=\cos \theta_{Y}+\eta$, which predicts the electrowetting behavior of a liquid droplet on dielectric layer. $\theta$ is the contact angle at an applied voltage $U ; \theta_{\mathrm{Y}}$ is the Young's equilibrium contact angle; and $\eta=$ $\varepsilon \varepsilon_{0} U^{2} / 2 d \sigma_{l v}$ is the dimensionless electrowetting number, where $\varepsilon$ is the dielectric constant of the dielectric material, $\varepsilon_{0}$ is vacuum permittivity, $d$ is the thickness of the dielectric layer and $\sigma_{\mathrm{v}}$ is the liquid/vapor interface energy. $\eta$ measures the strength of the electrostatic energy compared to surface tension. Similar electrowetting curves 
were obtained based on the Lippmann's equation for all three samples at $U<40 \mathrm{~V}$. The increased dielectric strength of dielectric layer leads to the inhibition of the saturation phenomenon ${ }^{[36]}$, and increases the breakdown voltage to result in lower saturation contact angle ${ }^{[37]}$. The higher contact angle saturation of RIE-reflow film is mainly caused by the weak dielectric strength due to the damages and impurities introduced during RIE and high temperature reflow processes. Deviation of the EW curves starts at lower voltage than the breakdown voltage to reach the saturation for both virgin and Reconstruction films. This might be explained by the charges adsorption/trapping on/in the AF surfaces. The trapped charges would partially screen the electric field, reducing the driving force on the liquid droplet, causing to reach the saturation contact angle at higher voltage ${ }^{[38]}$.
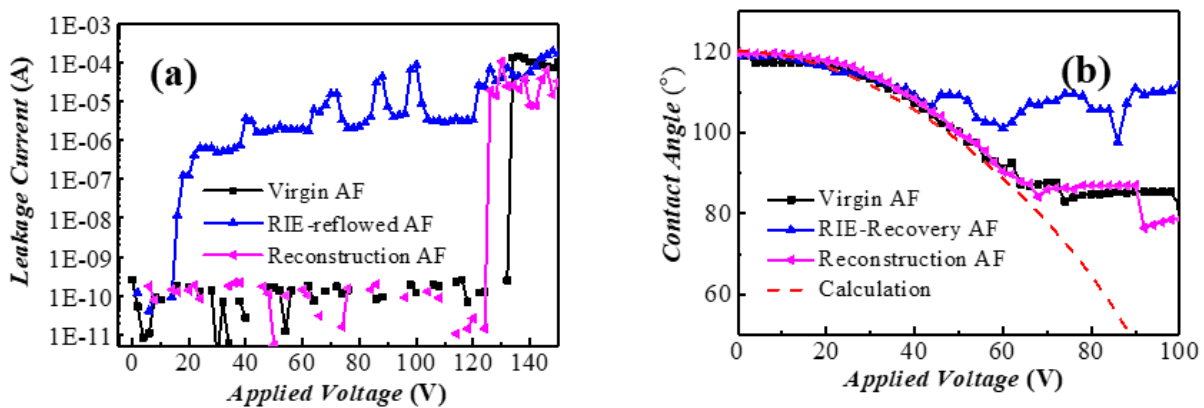

Figure 3.5 (a) Leakage current of the virgin, RIE-reflow processed and the Reconstruction processed AF films. (b) Electrowetting performance (contact angle varying with applied voltage) of the three films.

\subsubsection{Electrowetting devices based on the RC-HHPS platform}

The proposed reconstruction strategy for HHPS is highly valuable for practical applications in device construction since it is mainly based on standard film preparation and lithography. To demonstrate the practical usefulness of RC-HHPS, we use the process to fabricate an electrowetting display (EWD) device. EWD is a device utilizing a dual fluidic system of colored oil and transparent conductive liquid (water for instance) to realize display function in a microscale pixels based on the electrowetting mechanism ${ }^{[29]}$. Figure 3.6a shows the schematic drawing of a EWD pixel at "Off" and "On" states. In the absence of a voltage, the oil forms a continuous film in a pixel between the hydrophobic insulator-covered electrode and water, 
showing the color of the oil film the pixel. When a voltage is applied across the top and bottom electrodes, the transparent water is driven to move towards the insulator, pushing the oil aside to the wall, showing the color of the bottom substrate. In this way, the optical properties of the stack, when viewed from the top, are tuned between a colored Off-state (dyed oil) and a white On-state (color of bottom substrate) ${ }^{[15]}$.

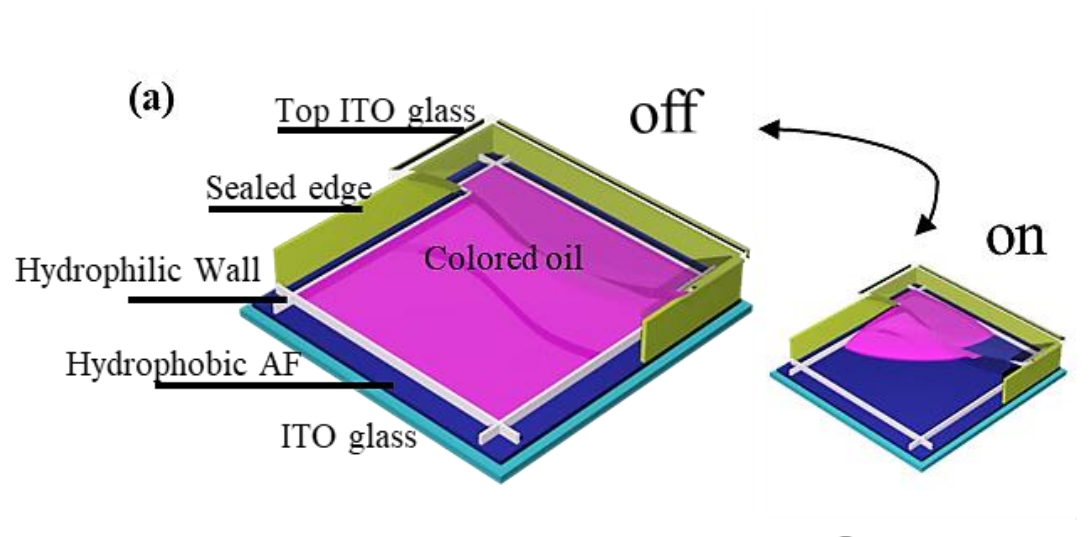

Off

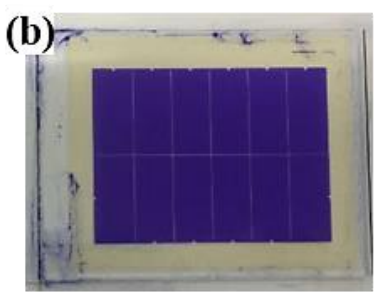

(d)

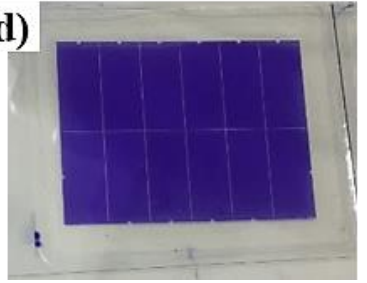

On

(c)
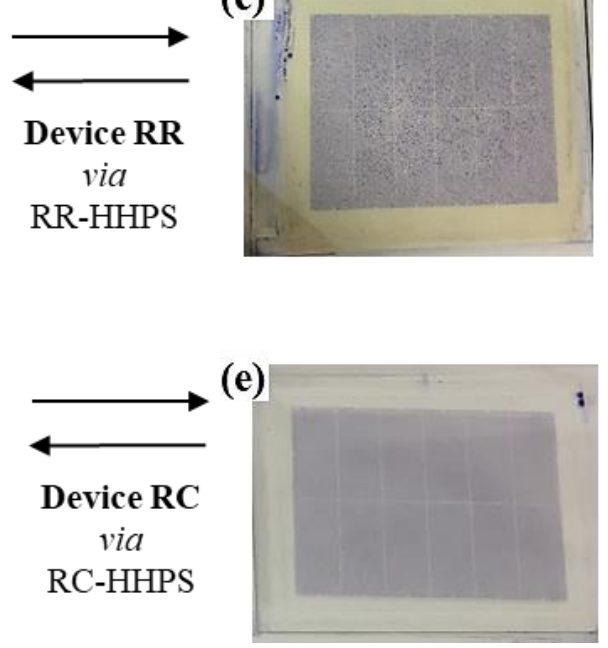

Figure 3.6 (a) Schematic of an electrowetting display pixel - principle and components. At the Off-state, in a EWD pixel, without applied voltage, a homogeneous oil film spreads over the pixel area showing the color of the dyed oil. at the On-state, in a EWD pixel, with an applied voltage, the oil film was pushed by water to one corner of the pixel, showing the color mostly from the bottom substrate. (b) and (c) are pictures of the Off-state and On-state (at 40 V DC voltage) of an EWD device fabricated using conventional RIE-reflow process based on the RR-HHPS (Device RR). (d) and (e) are pictures of the Off-state and On-state (at $40 \mathrm{~V}$ DC 
voltage) of an EWD device based on the RC-HHPS (Device RC). The display size is $50.7 \times$ $66.0 \mathrm{~mm}^{2}$ for both devices.

To evaluate the proposed strategy and materials, EWD devices (Figures 3.6b and 3.6d) were fabricated using the conventional RR-HHPS (Device RR) and this RC-HHPS (Device RC) processes. The EWD is $50.7 \times 66.0 \mathrm{~mm}^{2}$ in area, containing $1.48 \times 10^{5}$ pixels. The pixel pitch is $150 \mu \mathrm{m}$ with walls of $7 \mu \mathrm{m}$ high and $15 \mu \mathrm{m}$ wide. Except for the wall patterning, all other fabrication processes are the same as reported ${ }^{[13,15]}$. The hydrophilic materials utilized in the two devices are both SU-8 3005. As the high temperature reflow process was applied in the conventional RR-HHPS fabrication procedure, the color of SU-8 changed to light yellow. The color changing of the hydrophilic pixel walls somehow affects the display contrast of the devices.

Figures 3.7a and 3.7b show the curves of opening ratio depending on applied voltage of six adjacent pixels in Device RR and Device RC, respectively. The adjacent pixels are in 2 rows labeled as Nos. 1-6. The opening ratio of a pixel was extracted from the video snapshots using a MATLAB routine, as reported earlier ${ }^{[13]}$. It is obvious that the curves of Device RR is more random with larger variations in both the threshold voltage (pixel starts to open) and the maximum opening ratio. This is mainly due to the un-recovered non-uniform AF surface induced by RIE reaction. Non-uniform regions on the surface results in variations of the oil volume filled into each pixel ${ }^{[13]}$. This deviation, on one hand, influences the threshold voltage caused by the variation in oil film thickness. On the other hand, it affects the oil moving speed driving by water under EW driving, resulting in differences in opening speed and the maximum opening ratio. The threshold voltages vary in the range of 20-55 V for Device RR. Based on the overall observation, in Device RR, at $U=40$ and $45 \mathrm{~V}, 80 \%$ and $90 \%$ pixels started to open; oil overflow from one pixel to an adjacent one was observed frequently. "Overflow" here refers to the oil climb over pixel walls to the adjacent pixel and un-return afterwards. Inset images in Figure 3.7a show the opening states of the 6 pixels under various voltages. In Device RC, all six pixels start to open at $25 \mathrm{~V}$, and the opening ratio increases gradually with applied voltage and reach the maximum opening ratio of $\sim 0.7$ at $70 \mathrm{~V}$. As seen from the inset images, the pixels look homogeneous and similar. The opening style, ending position and size of the dye oil are also similar, proving their homogeneity and reliability. Over the whole device, more than $95 \%$ pixels start to open at $25 \mathrm{~V}$ without obvious defect pixels. And overflow is also completely eliminated. "Overflow" in a device results in empty pixels at Off-state. 
The final opening ratios seem to achieve similar level of about 0.7-0.8 in both Device RR and Device RC. However, in the Device RR, when the opening ratio reaches such a high level the oil would sit on or climb over the walls and flow into the adjacent pixel (namely overflow) due to the less hydrophilicity of the wall surfaces (see the inset of Figure 3.7a). Once the oil flows into the adjacent pixel, the oil from two pixels mixes together with one pixel empty and the other pixel overwhelm. As a result, the "overflow" induced defects would appear in the device. This means that the conventional Device RR could not achieve reversible switch at such a high opening ratio. However, in the Device RC, the same opening ratio could be repeatable achieved without obvious defects observed. Thus, the "overflow" phenomena could be avoided according to the super hydrophilic wall surfaces in the Device RC.
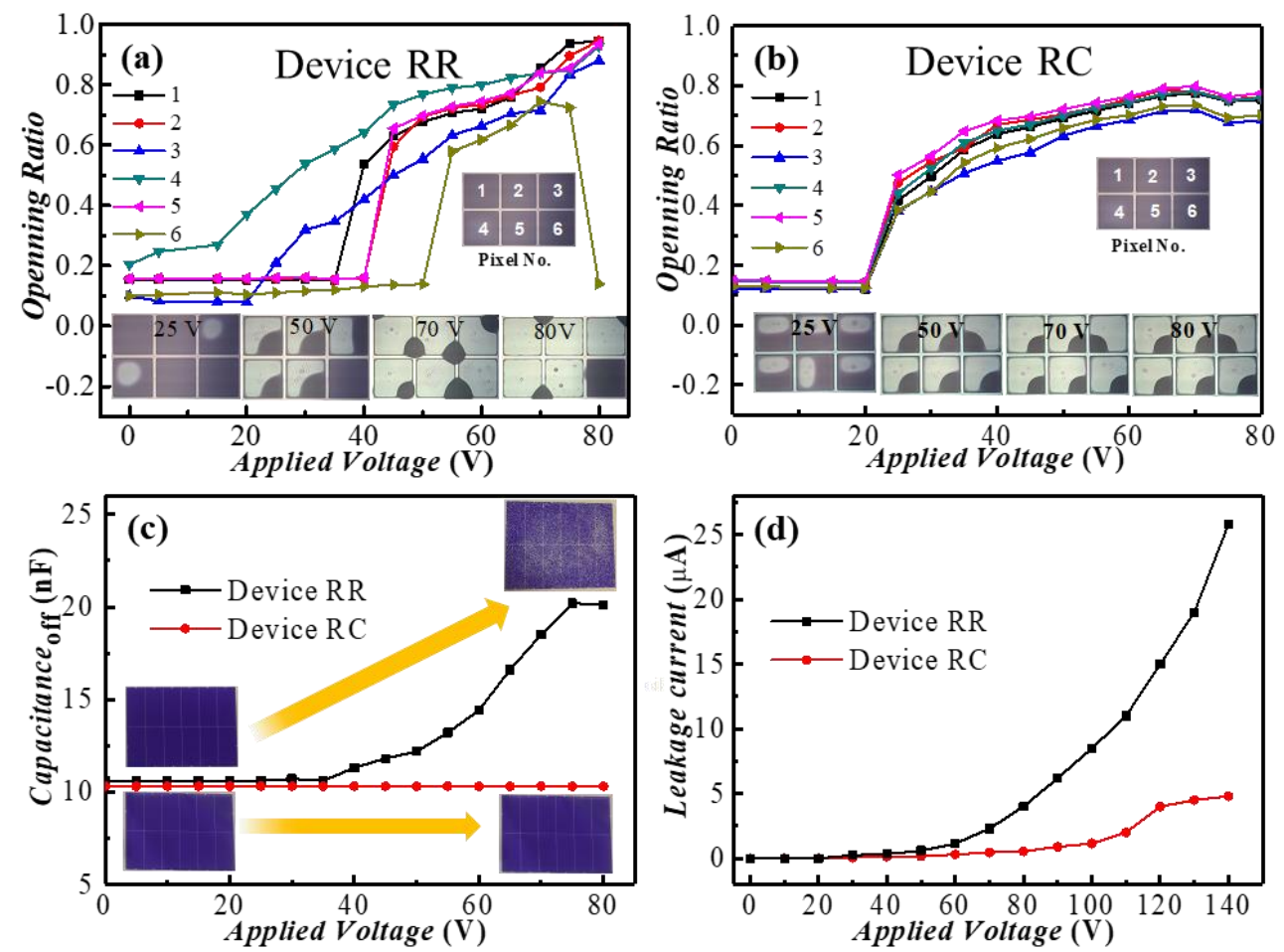

Figure 3.7 Opening ratio varying with applied voltage in EWD devices fabricated by (a) conventional RIE-reflow (Device RR) and (b) proposed Reconstruction processes (Device $\mathrm{RC}$ ). Insets are the microscope images of the $\mathrm{f} 3 \times 2$ pixels of Devices RR and Device RC. (c) Off-state capacitance (measured after switching off the electrical field) vs the applied voltage in Devices RR and RC. Insets are the corresponding images at 1 and $70 \mathrm{~V}$. (d) Leakage current versus applied voltage in Devices RR and RC. All pixel size is $150 \times 150 \mu \mathrm{m}^{2}$. 
As the oil film and AF layer form a series circuit, the capacitance in the Off-state starts to increase if there are AF surfaces without oil filling ${ }^{[39]}$. Figure $3.7 \mathrm{c}$ shows the Offstate capacitance measured after turning off the applied voltage in the range of 0-80 $\mathrm{V}$. The Off-state capacitance shows the percentage of the pixels lack of oil due to the "overflow" defects. In Device RR, the obvious increased Off-state capacitance at $U>40 \mathrm{~V}$, indicates the increase of the "overflow" pixels. In Device RC, the Off-state capacitance keeps constant without obvious change, indicating the stable and repeatable pixel switching behavior. The inset images of the Off-state devices at 0 and $70 \mathrm{~V}$ demonstrate obvious optical difference between two devices. The high wettability contrast between fresh $\mathrm{AF}$ and RIE-treated wall surfaces ensures the restrict confinement of oil inside the pixels. The leakage current $\left(I_{\text {leak }}\right)$ measurements of the two devices are shown in Figure 3.7d. $I_{\text {leak }}$ was recorded after each voltage being applied for $2 \mathrm{~min}$. $I_{\text {leak }}$ corresponding to the applied voltage was measured in a large range of 0-140 V. Overall, Device RC shows obviously lower $I_{\text {leak }}$ than Device RR. In Device RR, $I_{\text {leak }}$ starts to increase substantially at $50 \mathrm{~V}$, which suggests that the AF films in pixels starts to breakdown. However, in Device RC, $I_{\text {leak }}$ keeps stable when the applied voltage is lower than $100 \mathrm{~V}$. The obvious increase of $I_{\text {leak }}$ appears at 120 $\mathrm{V}$, suggesting the Teflon AF films in most pixels start breaking down at $120 \mathrm{~V}$. The AF film thickness is $\sim 800 \mathrm{~nm}$ for both Devices RR and RC. This confirms that the dielectric strength of RC-HHPS is much higher than that of RR-HHPS. $I_{\text {leak }}$ of Devices $\mathrm{RC}$ and RR, driven at $U=30 \mathrm{~V}$, are 0.07 and $0.25 \mu \mathrm{A}$, respectively; and $I_{\text {leak }}$ at $U=40$ $\mathrm{V}$ are 0.14 and $0.34 \mu \mathrm{A}$, respectively. The power consumption of the Device RC driven at commonly working conditions with applied voltages of 30 and $40 \mathrm{~V}$, was calculated to be 0.06 and $0.16 \mu \mathrm{W} / \mathrm{cm}^{2}$, respectively. These values are lower than the power consumption for Device RR of 0.23 and $0.39 \mu \mathrm{W} / \mathrm{cm}^{2}$ at the same working conditions with applied voltages of 30 and $40 \mathrm{~V}$, respectively. M. Fernández et al ${ }^{[40]}$ has divided energy consumption of displays into 4 levels as: high $\left(>250 \mathrm{~mW} / \mathrm{cm}^{2}\right)$, medium $\left(100-250 \mathrm{~mW} / \mathrm{cm}^{2}\right)$, low $\left(10-100 \mathrm{~mW} / \mathrm{cm}^{2}\right)$ and ultra-low $\left(0-10 \mathrm{~mW} / \mathrm{cm}^{2}\right)$. In this way, the display devices shown here belong to the ultra-low level, which is also highly beneficial for future applications as a green electronic device. This result on the device level is consist with the electrical measurements in the film level.

To further verify the feasibility of the proposed strategy for high quality EWD applications, we fabricated and integrated two EWD systems driven by the active matrix (Display I) and patterned ITO (Display II) backplanes by using the RC-HHPS substrate proposed in this work. Figure 3.8 shows the dynamic demonstration of images of lines and numbers on the active matrix and patterned ITO driven displays. 
The display areas are $7.5 \times 7.5 \mathrm{~cm}^{2}$ and $5.9 \times 3.0 \mathrm{~cm}^{2}$ for Displays I and II, respectively. All pixels are in square shape with size of $200 \times 200 \mu \mathrm{m}^{2}$. The driven voltage was set at $30 \mathrm{~V}$. All response time was measured to be $<50 \mathrm{~ms}$. Both devices fabricated by the proposed RC-HHPS show reliable and repeatable performance for high quality and dynamic image displaying.
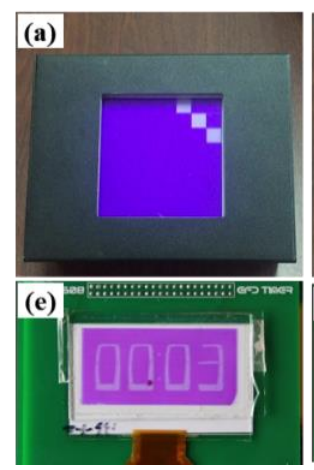
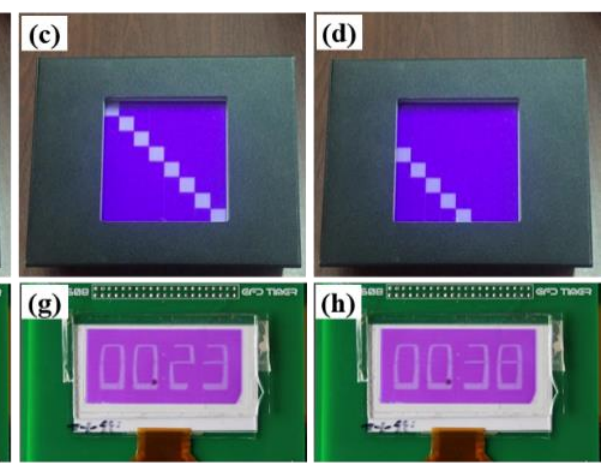

Figure 3.8 Images showing displaying squares in line on a EWD fabricated using the proposed Reconstructed (RC-HHPS) process driven by (a-d) an active matrix backplane (Display I) and (e-h) patterned ITO electrodes (Display II).

\subsection{Conclusion}

In this work, we propose and validate a reconstructive approach to fabricate HHPS with high wettability contract. The large oil/water contact angle difference of $175^{\circ}$ has been reached between the hydrophobic and hydrophilic patterned surfaces (RCHHPS). By utilizing this "reconstruction" method, the incomplete recovery issue in the conventional "RIE-reflow' process is avoid; as a result, the properties of original and fresh AF surfaces are retained which contribute to the high hydrophobicity and stable dielectric strength. On the other hand, the highly hydrophilic property is achieved on the wall surfaces according to the heavy RIE process to obtain chemically more hydrophilic groups and physically rough nano-features on surface. Electrowetting display devices are fabricated to verify the performance of the RCHHPS, and compared to RR-HHPS. The enhanced uniform pixel aperture ratio, voltage tolerance, and less leakage current of the EWD devices demonstrate the superiority of the RC-HHPS obtained using our proposed approach. Such a RC-HHPS process is highly valuable to construct surfaces with high wettability contrast, which could be widely applied to construct chemical, electrical and optical devices using conventional film materials and standard photolithography processes. 


\section{Acknowledgments}

The authors thank Yingying Dou and Guimei Qin for their training of the lithography process, Yuanyuan Guo for her support of the device assembly part, Shouming Li and Rui Zhou for the electrode design, and Weijie Lin for the electric driving part. This work has been financially supported by the National Key Research \& Development Program of China (2016YFB0401502), the National Natural Science Foundation of China (61574065, U1501244), Science and Technology Planning Project of Guangdong Province (2016B090906004), Special Fund Project of Science and Technology Application in Guangdong (2017B020240002), Guangdong Innovative Research Team Program (No. 2013C102), PCSIRT Project No. IRT_17R40, the National 111 Project, and the MOE International Laboratory for Optical Information Technologies.

\section{Contributions}

Hao Wu, Robert A. Hayes, Fahong Li and Guofu Zhou conceived this project. Hao $\mathrm{Wu}$ designed the experiments and performed the main experiments; Guofu Zhou and Fahong $\mathrm{Li}$ supervised this work; Hao $\mathrm{Wu}$ and Lingling Shui analyzed the data and wrote the manuscript; Fahong Li, Alex Henzen, Lingling Shui, Frieder Mugele and Guofu Zhou revised the manuscript.

\section{References}

[1] A. R. Parker, C. R. Lawrence, Nature 2001, 414, 33.

[2] R. Garrod, L. Harris, W. Schofield, J. McGettrick, L. Ward, D. Teare, J. Badyal, Langmuir 2007, 23, 689.

[3] L. Zhai, M. C. Berg, F. C. Cebeci, Y. Kim, J. M. Milwid, M. F. Rubner, R. E. Cohen, Nano Letters 2006, 6, 1213.

[4] T. Sun, L. Feng, X. Gao, L. Jiang, Accounts of chemical research 2005, 38, 644.

[5] J. Seo, S. Lee, J. Lee, T. Lee, ACS applied materials \& interfaces 2011, 3, 4722.

[6] V. Belova, D. A. Gorin, D. G. Shchukin, H. Möhwald, Angewandte Chemie International Edition 2010, 49, 7129.

[7] A. R. Betz, J. Xu, H. Qiu, D. Attinger, Applied Physics Letters 2010, 97, 141909. 
[8] L. Mishchenko, M. Khan, J. Aizenberg, B. D. Hatton, Advanced functional materials 2013, 23, 4577.

[9] M. Cao, J. Xiao, C. Yu, K. Li, L. Jiang, Small 2015, 11, 4379.

[10] J. Ju, H. Bai, Y. Zheng, T. Zhao, R. Fang, L. Jiang, Nature communications 2012, 3, 1247.

[11] Y. Lai, L. Lin, F. Pan, J. Huang, R. Song, Y. Huang, C. Lin, H. Fuchs, L. Chi, Small 2013, 9, 2945.

[12] W. G. Brodbeck, J. Patel, G. Voskerician, E. Christenson, M. S. Shive, Y. Nakayama, T. Matsuda, N. P. Ziats, J. M. Anderson, Proceedings of the National Academy of Sciences 2002, 99, 10287.

[13] T. He, M. Jin, J. C. Eijkel, G. Zhou, L. Shui, Biomicrofluidics 2016, 10, 011908.

[14] R. A. Hayes, B. J. Feenstra, Nature 2003, 425, 383.

[15] H. Wu, R. A. Hayes, F. Li, A. Henzen, L. Shui, G. Zhou, Displays 2018, 53, 47.

[16] H. Notsu, W. Kubo, I. Shitanda, T. Tatsuma, Journal of Materials Chemistry 2005, 15, 1523.

[17] H. Jeong, H. Moon, H.-J. Kim, M. Yoon, C.-G. Park, Y. S. Oh, H. J. Sung, D.-G. Choi, S. Yoo, ACS applied materials \& interfaces 2018, 10, 26501.

[18] H. Zhang, Q. Yan, Q. Xu, C. Xiao, X. Liang, Scientific reports 2017, 7, 3983.

[19] Y. Li, E. McKenna, W. Parkes, A. Pitt, A. Walton, Applied Physics Letters 2011, 99, 073703.

[20] A. R. Abate, A. T. Krummel, D. Lee, M. Marquez, C. Holtze, D. A. Weitz, Lab on a Chip 2008, 8, 2157.

[21] H. Wu, B. Tang, R. A. Hayes, Y. Dou, Y. Guo, H. Jiang, G. Zhou, Materials 2016, 9, 707.

[22] X. Chen, T. He, H. Jiang, B. Wei, G. Chen, X. Fang, M. Jin, R. A. Hayes, G. Zhou, L. Shui, Displays 2015, 37, 79.

[23] L. R. Scarratt, B. S. Hoatson, E. S. Wood, B. S. Hawkett, C. Neto, ACS applied materials \& interfaces 2016, 8, 6743.

[24] D. Kim, A. Steckl, Langmuir 2010, 26, 9474.

[25] A. Nijdam, A. Monica, A. Gadre, J. Garra, T. Long, C. Luo, M.-C. Cheng, T. Schneider, R. White, M. Paranjape, Sensors and Actuators A: Physical 2005, 120, 172.

[26] Q. Chen, Journal of Physics D: Applied Physics 2004, 37, 715.

[27] H. Lee, K. Lee, B. Ahn, J. Xu, L. Xu, K. W. Oh, Journal of Micromechanics and Microengineering 2011, 21, 125006. 
[28] N.-G. Cha, Y. Echegoyen, T.-H. Kim, J.-G. Park, A. A. Busnaina, Langmuir 2009, 25, 11375.

[29] Y. Xi, H. Guo, Y. Zi, X. Li, J. Wang, J. Deng, S. Li, C. Hu, X. Cao, Z. L. Wang, Advanced Energy Materials 2017, 7, 1602397.

[30] C. J. Drummond, G. Georgaklis, D. Y. Chan, Langmuir 1996, 12, 2617.

[31] F. Walther, P. Davydovskaya, S. Zürcher, M. Kaiser, H. Herberg, A. M. Gigler, R. W. Stark, Journal of Micromechanics and Microengineering 2007, 17, 524.

[32] L. Martin, H. Martinez, M. Ulldemolins, B. Pecquenard, F. Le Cras, Solid State Ionics 2012, 215, 36.

[33] S.-J. Ding, P.-F. Wang, X.-G. Wan, D. W. Zhang, J.-T. Wang, W. W. Lee, Materials Science and Engineering: B 2001, 83, 130.

[34] G. Lloyd, M. Raja, I. Sellers, N. Sedghi, R. Di Lucrezia, S. Higgins, B. Eccleston, Microelectronic engineering 2001, 59, 323.

[35] X. Guo, T. Ma, IEEE Electron Device Letters 1998, 19, 207.

[36] A. Papathanasiou, A. Papaioannou, A. Boudouvis, Journal of Applied Physics 2008, 103, 034901.

[37] A. Grisatya, Y. H. Won, presented at Oxide-based Materials and Devices V 2014.

[38] H. Verheijen, M. Prins, Langmuir 1999, 15, 6616.

[39] H. Wu, B. Tang, R. Hayes, Y. Dou, Y. Guo, H. Jiang, G. Zhou, Materials 2016, 9, 707.

[40] M. Fernández, E. Casanova, I. Alonso, Sustainability 2015, 7, 10854. 


\section{Chapter 4}

\section{Electrically controlled localized charge trapping at amorphous fluoropolymer-electrolyte interfaces*}

Charge trapping is a long-lasting problem in electrowetting-on-dielectric (EWOD), causing reliability reduction and restricting its practical applications. In this chapter, charge trapping induced by electrowetting (EW) at high voltage is revealed to be localized in the vicinity of the three-phase contact line using local contact angle measurements, EW-microprobe, and Kelvin Probe Force Microscopy (KPFM). While a drawback for most conventional EW applications, we demonstrate this EW-assisted Charge Injection (EWCI) can serve as a simple and low-cost method to deposit stable charge patterns on fluoropolymer surfaces with microscale resolution.

\footnotetext{
* This chapter is based on publication: H. Wu, R. Dey, I. Siretanu, D. van den Ende, L. Shui, G. Zhou, F. Mugele, Electrically controlled localized charge trapping at amorphous fluoropolymer-electrolyte interfaces, Small, (2019) 1905726.
} 


\subsection{Introduction}

Amorphous fluoropolymers (AFPs) are popular materials for various applications ${ }^{[1-5]}$ because of the unique combination of favorable material properties such as chemical inertness, mechanical strength, water repellency, dielectric strength, optical transparency, and easy solution processability ${ }^{[5-6]}$. For these reasons, AFPs are also predominantly used as insulating and hydrophobic layer in electrowetting (EW) devices ${ }^{[6-11]}$. EW, which is often also denoted as 'electrowetting-on-dielectric' (EWOD) to emphasize the relevance of the dielectric layer, relies on the fact that ionic charge carriers have in general a rather low affinity to the weakly polarizable AFPs. However, at the same time, fluoropolymers have been used for decades as charge storage (electret) materials with applications in electro-mechanical transductions, such as microphones, micro-electro-mechanical systems (MEMS) and electric generators ${ }^{[12-14]}$. These applications rely on the fact that charges, once deposited on or within AFPs, remain stable in the materials. The purpose of the present work is to shed light on these two contradictory aspects of charge repellence and charge storage in AFPs that together control the extent of charge injection into AFPs during EWOD at high voltage. Such EW-assisted charge injection (EWCI), if done in a controllable way, can be used as an innovative method for generating permanent charge patterns on AFPs.

The reliability of any EW applications in microfluidics ${ }^{[15-16]}$, optofluidics ${ }^{[17-18]}$, display technology ${ }^{[19-20]}$, and energy harvesting ${ }^{[21]}$ relies on the reproducibility, performance, and durability of the dielectric layer, and hence, the stability of AFPs is particularly important ${ }^{[22-25]}$. Charge trapping at the dielectric/electrolyte interfaces is a long-stand problem in EW, leading to the degradation of the electrical response of AFP films, and thus causing the contact angle saturation and failures in EWOD devices ${ }^{[21,26-27]}$. While early experiments with composite dielectrics displayed a reversible response and symmetric saturation for positive and negative bias voltage, suggesting substantial mobility of both types of charge carriers upon injection into the AFP films ${ }^{[28]}$, more recent studies displayed a strongly asymmetric and sometimes irreversible response $^{[29],[30]}$. It was also reported that AFP materials even spontaneously assumed a permanent negative surface charge upon extended (several hours) contacting with water in the absence of any applied voltage ${ }^{[31]}$.

Since most studies on EW-induced charge trapping phenomena mainly focus on the response of the macroscopic contact angles and the total electrical currents, there is a significant lack of a quantitative description of the underlying microscopic charge 
trapping phenomenon. Moreover, a clear understanding of the correlation between the charge trapping and the macroscopic wetting characteristics has still remained elusive. It was recognized that diverging electric fields in the vicinity of the three phase contact line (TPCL) cause various types of non-linear response of the materials during EW, which may limit the minimum contact angle ${ }^{[32-33]}$. However, whether the heterogeneity of the electric field actually leads to the charge trapping and induces permanent changes in the local surfaces of AFPs is still unclear. The charge injection process was assumed to essentially follow the distribution of the electric field with its well-established divergence near the TPCL ${ }^{[34-35]}$. However, several recent studies on charge injection during EWOD using local surface potential measurement with noncontact electrostatic probes, show otherwise ${ }^{[27,36-37]}$. Surprisingly, the measured surface potential distributions were reported to be rather broad with a maximum in the center of the droplet, thereby challenging the classical view based on the local field divergence ${ }^{[27,36-37]}$.

In this work, we analyze the charge distribution generated on the Teflon AF (a typical AFP material) surfaces by EW at high voltage with unprecedented lateral resolution, and explore the usage of the EWCI method for localized charge deposition at AFP surfaces. Three complementary techniques are used to reveal the local charge distribution on single layer AFP surfaces. We quantitatively demonstrate that EWinduced charge injection via a water drop is highly localized. Based on this, a simple and low-cost approach is proposed and validated to generate stable charge patterns with controllable line-width and charge density. As a result, we can tune the surface properties of AFP by EWCI at a microscale level without complex microfabrication processes involving high-end instruments. The excellent stability of the negative trapping charges, in particular in a "harsh" environment under water or high humidity, demonstrates the potential of the proposed methodology for a wide range of applications requiring stable surface charges ${ }^{[38-42]}$.

\subsection{Results and discussion}

\subsubsection{Evidence of charge trapping phenomenon}

\section{Macroscopic surface wettability upon charging}

The working principle of EWOD is schematically shown in Figure 4.1a. When a voltage $(U)$ is applied on the dielectric layer via a electrolyte droplet and the bottom electrode, a pulling force $\left(f_{U}\right)$ derived from the applied electric field pulls the TPCL 
toward the outside direction of the droplet, and thus changes the contact angle. This pulling force $f_{U}=\frac{\sigma_{S}{ }^{2}}{2 c}$ is governed by the total amount of charges at the electrolyte/solid interfaces $\sigma_{S}$ (the same amount of counter charges are in the bottom electrode) and the capacitance per unit area of the dielectric layer (c). When $U$ is relatively low, there is no 'trapping charge' at the electrolyte/solid interfaces. All surface charges are supposed to be contributed by the electric field, and the pulling force is $f_{U}=\frac{1}{2} c U^{2}$. Thus, the contact angle $\theta(U)$ is given by the classical YoungLippmann EW model:

$$
\cos \theta(U)=\cos \theta_{Y}+\frac{c}{2 \gamma} U^{2}
$$

where $\theta_{Y}$ is Young's angle, $\gamma$ is the surface tension of the liquid and $U$ is the applied voltage. The contact angle response under the applied voltage is shown in Figure S4.1. However, when the voltage reaches a certain high value, charges can be trapped at the electrolyte/solid interfaces, thus causing contact angle saturation ${ }^{[28]}$. The charge trapping process could be reversible or irreversible. Once the charges are irreversibly trapped in the dielectric layer, the electric response of the dielectric layer will be permanently degraded ${ }^{[21,30]}$.

To investigate the charge trapping phenomenon in EWOD, we intentionally achieve the contact angle saturation by abruptly applying a voltage as high as $-120 \mathrm{~V}$ to the EWOD system. As a response, the drop spreads within a few tens of ms from the initial contact angle of $\sim 115^{\circ}$ to $\sim 70^{\circ}$ (Figure 4.1b). Subsequently, a slow relaxation to $\theta\left(U_{c}\right) \approx 80^{\circ}$ takes place for approximately $1 \mathrm{~min}$. Along with the increase in contact angle, the radius of footprint area of the drop decreases (Figure 4.1c). This macroscopic contact angle retreat phenomenon has also been observed in previous works ${ }^{[23,29,36]}$. Because of the humid environment, we know that this relaxation is not caused by evaporation (Figure S4.2). After $300 \mathrm{~s}$, when the charging voltage is turned off, subsequent inspection of the samples by optical and by atomic force microscopy does not display any appreciable variation of the surface topography.

As discussed above, the contact angle variation in EW is the joint effect of the materials' surface (interface) tension, the applied voltage, the reversible and the irreversible trapping charges. In the present experiment, the material surface (interface) tension and the applied voltage ( $U=-120 \mathrm{~V}$ ) are kept constant, and the pulling force contributed by the applied voltage is $f_{U}=\frac{1}{2} c U^{2}=158 \mathrm{mN} / \mathrm{m}$. Due to the effect of trapping charges, the pulling forces are suppressed to $\gamma_{w / a}\left(\cos 115^{\circ}-\right.$ 
$\left.\cos 70^{\circ}\right)=55 \mathrm{mN} / \mathrm{m}$ for the initial contact angle of $70^{\circ}$ to $\gamma_{w / a}\left(\cos 115^{\circ}-\right.$ $\left.\cos 80^{\circ}\right)=43 \mathrm{mN} / \mathrm{m}$ for the final contact angle of $80^{\circ}$ (water/air interfacial tension $\gamma_{w / a}=72 \mathrm{mN} / \mathrm{m}$ ). The $12 \mathrm{mN} / \mathrm{m}$ reduction of pulling force is supposed to be caused by the charge density variation of both reversible and irreversible charge trapping during this charging process.

(a)

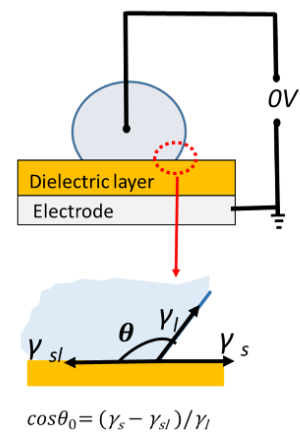

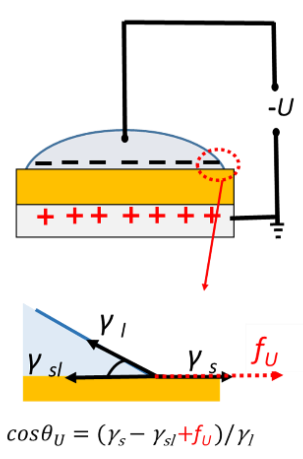

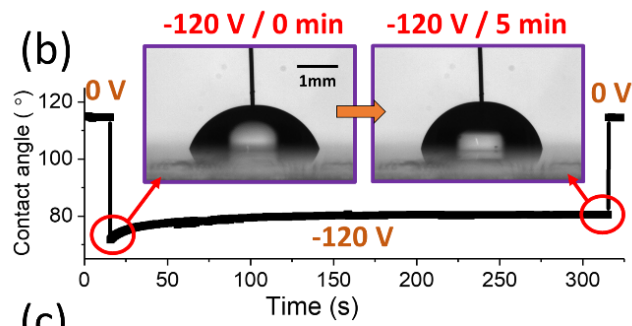

(c)

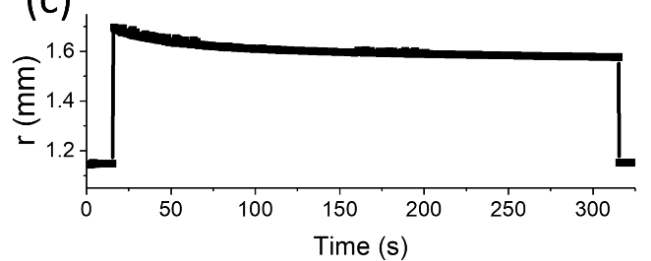

Figure 4.1. (a) Illustration of the electrowetting on dielectric (EWOD) principle. $\gamma_{s}, \gamma_{l}$ and $\gamma_{s l}$ are the solid/air, liquid/air and solid/liquid interface tensions. $f_{U}$ is a pulling force under the applied voltage of $U$. (b) Water/air contact angle and (c) contact line radius of a $5 \mu \mathrm{L}$ water droplet depending on time with $-120 \mathrm{~V}$ voltage applied. Insets of b) show side view images of charging droplets immediately after applying the voltage and after $5 \mathrm{~min}$.

\section{Local contact angles}

To further investigate whether the charge trapping is reversible and occurs over the entire drop-substrate interface, we remove the charging drop (after turning off the charging voltage) and subsequently investigate the surface properties in several manners. First, the wettability of the surface is investigated with high lateral resolution using a contact angle measurement with a much smaller probe droplet $(0.3 \mu \mathrm{L})$ (the setup shown in Figure S4.3). To minimize disturbing effects of contact angle hysteresis, these measurements are carried out in ambient oil. Figure 4.2a shows a composite side view image of such a probe droplet at various locations on the surface. The probe drop is always in contact with the electrically grounded Pt wire, guaranteeing zero potential drop between the drop and the electrode on the substrate. 
Figure 4.2b shows a one-dimensional variation of $\theta(U=0)$ with position along a diameter of the charging droplet footprint; Figure 4.2c shows a full two-dimensional map of the reduction of $\theta(U=0)$ all along the contact line of the original charging drop. Away from the position of the charging drop, the contact angle of the probe drop is close to $170^{\circ}$. In the center of the charging drop, the same contact angle is observed within the resolution of this measurement. In contrast, in the region close to the contact line during charging, $\theta(U=0)$ is reduced to about $155^{\circ}$. The width of this region with reduced contact angle is approximately 0.2 to $0.5 \mathrm{~mm}$. The variation of the contact angle corresponds to a pulling force of $-\Delta f_{o}=\gamma_{w / o}\left(\cos 155^{\circ}-\right.$ $\left.\cos 170^{\circ}\right)=0.08 \gamma_{w / o} \approx 3.2 \mathrm{mN} / \mathrm{m}$ (water/oil interfacial tension $\gamma_{w / o}=41 \mathrm{~mJ} /$ $m^{2}$ ). Assuming $\Delta f_{o}$ is contributed by the trapped charges- $\Delta f_{o}=\sigma_{T}{ }^{2} / 2 c$, the surface charge density can be estimate to be around $0.37 \mathrm{mC} / \mathrm{m}^{2}$.
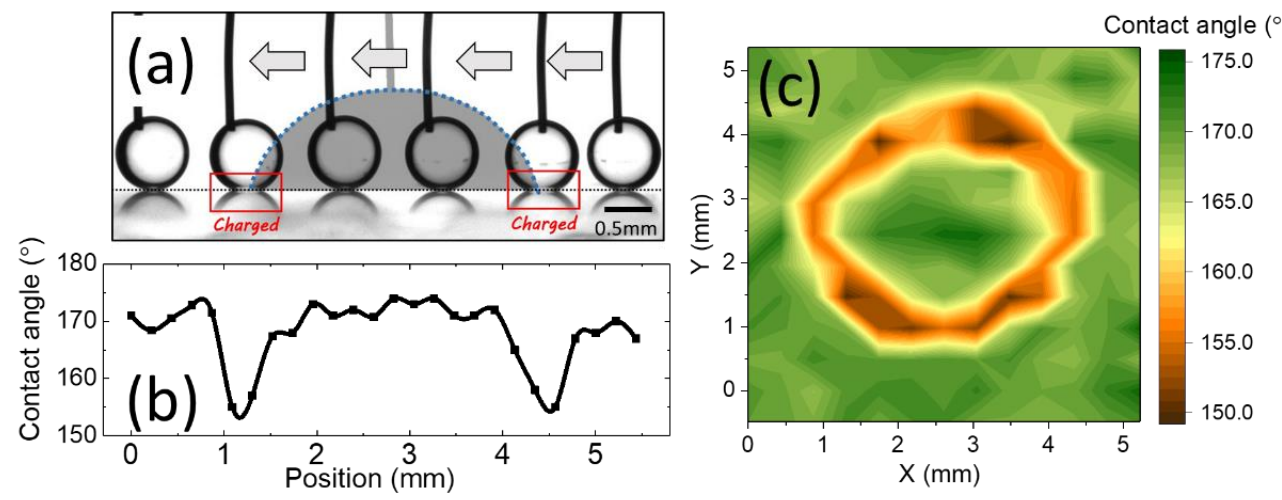

Figure 4.2. Contact angle reduction along contact line of charging drop. (a) Snapshots of electrically grounded probe-droplets $(V=0.3 \mu L)$ at different locations relative to the original charging droplet (grey background; $V=5 \mu L)$ that was used to charge the surface $\left(U_{c}=\right.$ $\left.-120 \mathrm{~V} ; t_{c}=5 \mathrm{~min}\right)$. (b) Contact angle $\theta(U=0)$ of electrically grounded drop vs. position extracted from (a) . (c) 2D map of $\theta(U=0)(12 \times 13$ locations $)$ as measured by probe drop.

\section{Electrowetting response}

With the trapping charges existing on a dielectric surface, the EW response curves follows a modified electrowetting equation ${ }^{[28,31]}$ of

$$
\cos \theta(U)=\cos \theta_{Y}+\frac{c}{2 \gamma}\left(U-U_{T}\right)^{2}
$$


where $U_{T}$, denoted as the 'trapping' voltage, corresponds to the potential on the droplet when it contains zero charge (i.e. all the counter charges induced by the trapping charges are remain in the bottom electrode). The observation of a finite offset voltage $U_{T}$ directly points to the presence of a finite permanent surface charge density of

$$
\sigma_{T}=c U_{T}
$$

at the polymer-electrolyte interfaces ${ }^{[28,31]}$.
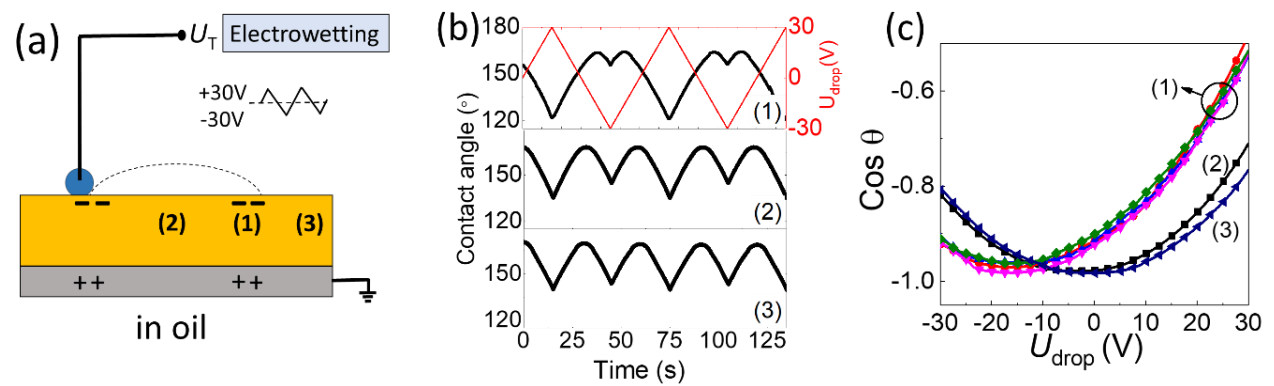

Figure 4.3. (a) Schematic of measuring trapping charge by electrowetting probe with different probing regions (1) contact line; (2) drop center; (3) pristine surface. The dashed line represents the shape profile of the previous electrolyte drop used during the EWCI process. This drop was removed immediately after the EWCI process. (b) EW response curves and (c) contact angle vs. applied voltage ( $U_{\text {drop }}$ ) of all three regions. (charging conditions: $-120 \mathrm{~V}$ for $5 \mathrm{~min}$ ).

The reduction of $\theta(U=0)$ presented in Figure 4.2 alone does not clearly indicate whether the effect is caused by local chemical variation of the surface along the contact line, which would reduce Young's angle $\theta_{Y}$, or whether it is indeed caused by the expected injection of surface charge, which would give rise to a finite value of trapping voltage $U_{T}$. To distinguish between the two scenarios, we perform EW measurements using probe droplets $(0.5 \mu \mathrm{L})$. The asymmetry of EW curve is found to be strongly position-dependent on the surface, being much more pronounce close to the contact line during charging, Region 2 in Figure 4.3, as compared to the central part of the charging drop (Region 1). Fitting the Equation 4.2 to the data shown in Figure $4.3 \mathrm{c}$, the trapping voltages are of $U_{T}(1)=-16 \mathrm{~V}, U_{T}(2)=-3 \mathrm{~V}$ and $U_{T}(3)=-1 V$ in Regions 1,2 and 3, respectively. The calculated $\sigma_{T}$ according to Equation 4.3 are $0.34,0.06$ and $0.02 \mathrm{mC} / \mathrm{m}^{2}$ in Regions 1,2 and 3, respectively. $\sigma_{T}$ 
of $0.34 \mathrm{mC} / \mathrm{m}^{2}$ at Region 1 is consistent with the calculated values from the local contact angle $\theta(U=0)$ variation in Figure 4.2 , which is $0.37 \mathrm{mC} / \mathrm{m}^{2}$. The consistancy of $\sigma_{T}$ value calculated from these two methods indicates that the reduction of $\theta(U=0)$ in Figure 4.2 is indeed induced by the irrevesible charge trapping, instead of the chemical surface modification. Hence, the AFP surface modification is caused by the deposition of charges during EW rather than by any chemical modification.

\section{Kelvin Probe Force Microscopy (KPFM) measurement}

Considering the fact that the probe droplets spread upon applying the voltage, and thus their footprint area increases quickly, one may wonder whether the probe drop remains within the narrow ring around the original contact line where the deposited charges are presumably trapped. If the probe drop spreads beyond the charging area, the measured asymmetry of the EW response and the value of $U_{T}$ would in part reflect the finite lateral extent of the deposited charge pattern rather than its absolute value.

To overcome the resolution limitation of contact angle-base detecting method, we performed Atomic Force Microscopy (AFM) and Kelvin Probe Force Microscopy (KPFM) measurements on the prepared charged Teflon surfaces in ambient air after removing the charging drop without immersing the surface into oil. The sample is charged by $-90 \mathrm{~V}$ for $5 \mathrm{~min}$. The AFM topography images display a very smooth surface with a roughness of a few nanometers. No indications of topographic surface modifications due to the charging process could be identified. In contrast, the KPFM measurements indeed reveal strong lateral variations of the surface potential $U_{S}$ in the region of the contact line, as shown in Figure 4.4. Overall, the KPFM measurements confirm the important observations of the macroscopic surface characterization (Figures 4.2 and 4.3). $U_{S}$ is essentially constant and small in the center of the charging drop-substrate interface. Pronounced variations of $U_{S}$ occurs in the rim along the contact line of the original charging drop. This rim is around $200 \mu \mathrm{m}$ to $300 \mu \mathrm{m}$ wide, only slightly smaller than that suggested by the contact angle and EW response measurements. The absolute value of the local surface potential in the KPFM measurements is around $-10 \mathrm{~V}$ which is consistent with the EW-response measurements at the same charging conditions (shown in Figure 4.5a and S4.5). Nanosized KPFM probe in ambient air and macroscopic EW-probed drops in ambient oil thus experience the same surface charge density $\sigma_{T}$, which could be obtained from the 
measured voltages using Equation 4.3 with $U_{T}=U_{S}$ (The detailed calculation of detecting surface potential $U_{S}$ and trapping voltage $U_{T}$ by KPFM can be found in S.I and Figure S4.4). Thus far, these results from three types of micro- and nano-scale measurements reveal and confirm that the charges are indeed trapped at the AFP surfaces after EW process and accumulate at the TPCL regions. These results also indicate that for EW applications driven by DC voltage, failures such as charge trapping or film break-down are more likely to occur at the TPCL region. Consequently, protecting the TPCL region may help to improve the quality and reliability of such EW devices.
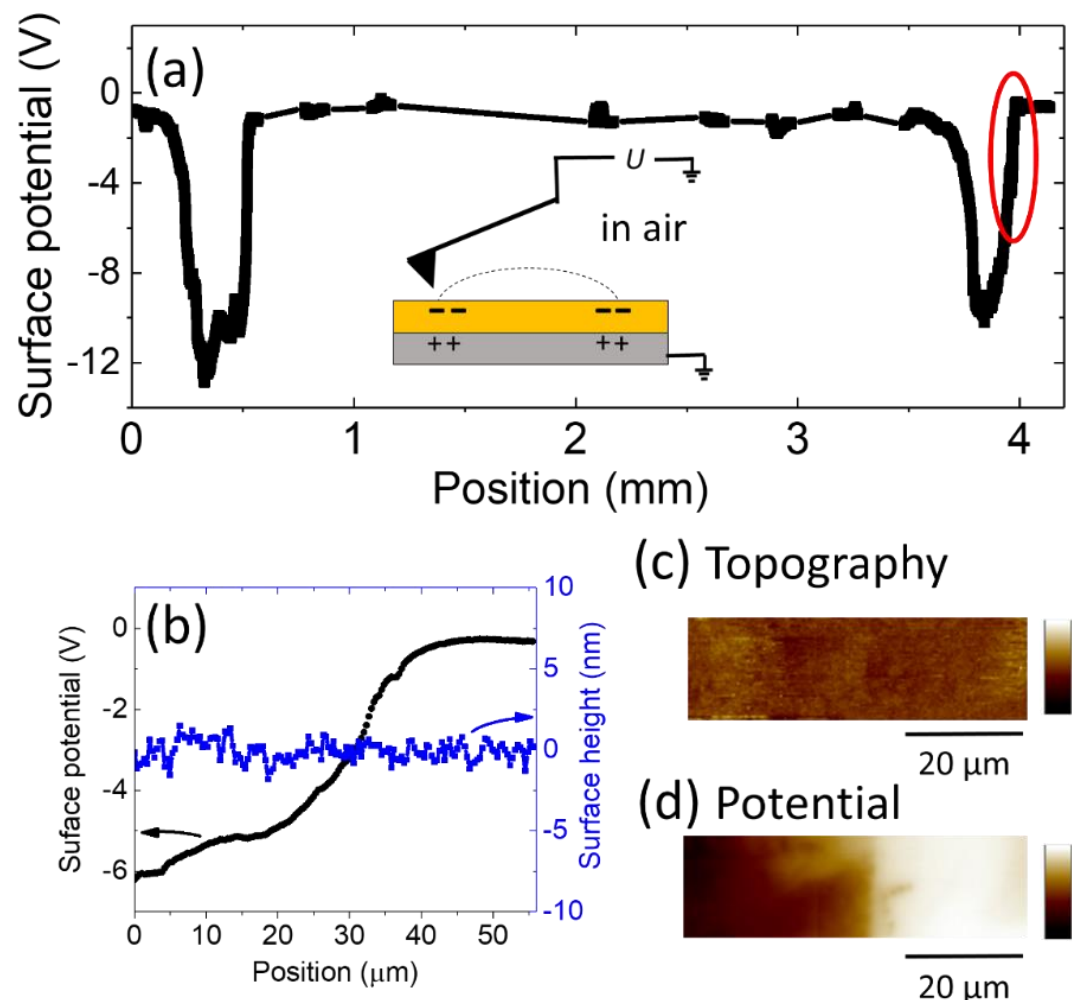

(c) Topography

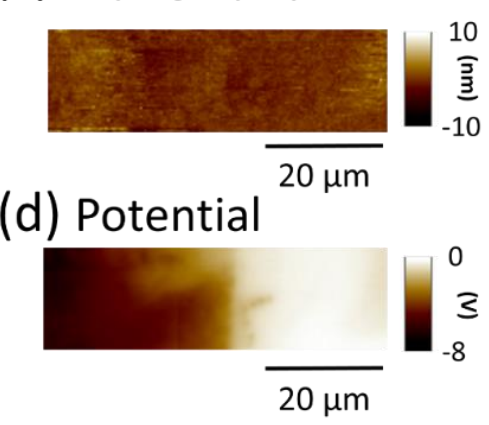

Figure 4.4. (a) Surface potential map measured by stitching several KPFM measurements of $90 \times 90 \mu^{2}$. (charging conditions: $-90 \mathrm{~V}$ for $5 \mathrm{~min}$ ) (b) Comparison of surface potential (black) and surface height (blue) in the region highlighted on the right in (a). (c) The surface topography and (d) local surface potential $U_{S}$ in the same region as in (b). 


\subsubsection{Controlling charge trapping by EWCI}

Having established and quantitatively characterized EWCI, we proceed to explore how to control density and distribution of the injected charge by our approach can lead to a novel, simple and low-cost method of printing controllable surface charges. Such a controllable surface charges are favored in many studies, such as energy harvesting ${ }^{[38,43-44]}$, super capacitors ${ }^{[45-46]}$, transport of droplets ${ }^{[42]}$, nanofluidics or nanoparticles ${ }^{[47-48]}$, water deionization ${ }^{[49]}$, antifouling ${ }^{[50]}$, and protein adsorption ${ }^{[51]}$.

\section{Controlling the maximum charge density}

To optimize the local surface charge density within the rim, we vary the applied voltage and duration for injecting charges, as well as the polarity of the voltage (Figure 4.5 and Figure S4.5). For a fixed charging time of $5 \mathrm{~min}$, the highest charge density of $0.37 \mathrm{mC} / \mathrm{m}^{2}$ is achieved at the highest negative charging voltage of $-140 \mathrm{~V}$. (The application of higher voltages is hampered by risk of dielectric breakdown. The dielectric strength of the dielectric films is shown in Figure S4.6). For a safe charging voltage of $-120 \mathrm{~V}$, the highest charge density as obtained approaching $\sigma_{\max }=$ $-0.46 \mathrm{mC} / \mathrm{m}^{2}$ after $20 \mathrm{~min}$. These results indicate that 10 times higher charge density than that of the spontaneous charges ${ }^{[31]}$ is reached, and almost hundred times faster than that from spontaneous charge accumulation at Telfon AF surfaces in contact with water ${ }^{[31]}$. This demonstrates the power of electric fields in immobilizing charge carriers at Teflon-water interfaces.

Negative charges deposited on the surface are very stable. No appreciable signs of degradation are observed even after 12 hours of "harsh" testing by continuous probing with a water drop on top of the surface with trapping charges (Figure 4.5c). After 36 $\mathrm{h}$ of immersion in oil, the measured negative charge density is still not altered. We also exposed the charged AFP films to water vapor in a closed chamber for $3 \mathrm{~h}$. As shown in Figure S4.7, even after this treatment the contact angle in the charged region is still significantly lower than elsewhere, confirming once again the stability of the trapped charges. 

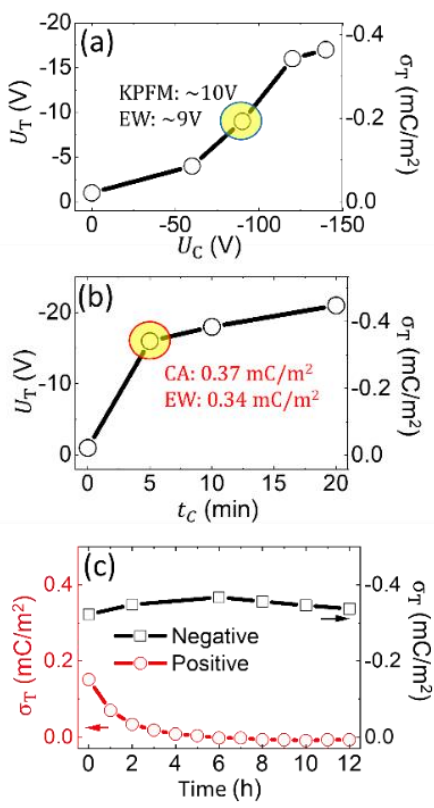

(d)
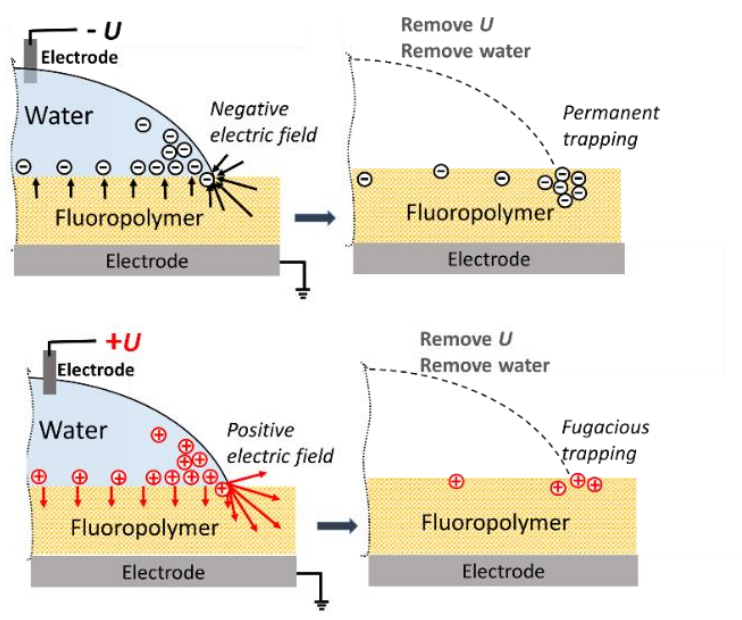

Remove $U$

-..... Remove water

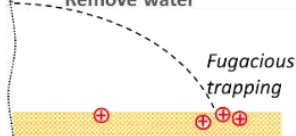

Fluoropolymer

Electrode

Figure 4.5. Trapping voltage $U_{T}$ and trapped charge density $\sigma_{T}$ based on EW measurements as a function of (a) charging voltage $\left(U_{C}\right)$ at fixed charging time of 5 min and (b) charging time at fixed charging voltage $\left(U_{C}=-120 \mathrm{~V}\right)$. Comparison of $U_{T}$ and $\sigma_{T}$ measured by $\mathrm{EW}$, KPFM and local contact angle (CA) of the samples charged at the same conditions are highlighted in (a) and (b). (c) Comparison of the trapping charge density as a function of time (30 V amplitude, 60s period; continuous measurement) between samples charged by $-120 \mathrm{~V}$ (black) and $+120 \mathrm{~V}$ (red). The EW curves for (a) and (b), and the corresponding $U_{T}$ information for (c) are shown in Figure S4.5. (d) Illustration of charge trapping process at Teflon surface. The black and red arrows indicate the electric field.

In contrast, for the opposite polarity, only half the maximum (positive) charge density could be deposited. More importantly, positive charges are unstable (Figure 4.5c) and relax within a few hours of continuous probing with a water drop in ambient oil. Due to this lack of stability, we did not explore positive charges in more detail.

Figure 4.5d summarizes these observations: When a relative high voltage is applied in EWOD systems, the charges accumulate at the TPCL region during charging process due to the local wedge shape of liquid and the fringe effect. After tuning off the voltage and taking away the electrolyte droplet, the negative charges are stably trapped in the previous TPCL region with a tunable charge density depending on the charging conditions. In contrast, the positive charges are only temporarily trapped on 
the polymer surfaces, suggesting that the traps for positive charges are shallower than those for negative ones.

This discussion raises the question about the nature and origin of the trapped surface charge. This question is probably closely related to the long-standing debate about the charge of hydrophobic-water interfaces on the colloidal scale ${ }^{[52]}$. Extensive studies (e.g. titration, electrokinetics) have demonstrated that such interfaces preferentially carry negative charge. Based on the widely observed increase with increasing $\mathrm{pH}$, it is believed that the charge density is caused by adsorbing hydroxide ions ${ }^{[53-56]}$. Yet, molecular dynamic (MD) simulations suggested that hydronium ions adsorb more strongly and sum frequency generation (SFG) spectroscopy failed to detect the expected $\mathrm{OH}$-stretch vibrations ${ }^{[52]}$. The latter leads to theoretical models predicting partial charge transfer. Our current measurements do not provide direct answers to this puzzle. Nevertheless, they do support the stronger affinity of negative charges to the interface, consistent not only with electrokinetics and the earlier report on spontaneous water-aged AFP surfaces ${ }^{[31]}$, but also with the better stability of negative charges in corona-charged electrets ${ }^{[57]}$. Since we did not intentionally add any salt, the only anions present in our solution should be hydroxide ions. It may well be that these ions donate their excess electron to polymer surface leading to similar trapped electronic states as commonly used to describe corona-charged electret materials. Note, however, the observed charge densities are very low, corresponding to a few hundred square nanometers per unit charge. As a consequence, attempts to detect them spectroscopically by XPS and thus determine their chemical nature have remained elusive $^{[58]}$.

\section{Creating narrow charge distributions}

According to the classical EW theory ${ }^{[34-35]}$, the high electric charge densities should be localized within a region of the order of the thickness of the dielectric layer. These enhanced local electric charge densities and the corresponding electric fields are supposed to be responsible for the charge injection. Following this thought, the intrinsic charge generation mechanism should allow to generate much narrower charge distribution region than the measured width of 200 to $300 \mu \mathrm{m}$. A solution of the electrostatic problem adapted to the parameters of the present experiments shows that the region, in which the local electric field exceeded the average field $U_{c} / d$ under the charging drop by more than a factor of two, is less than $1 \mu \mathrm{m}$ in width, as shown 
in Figure 4.6. We attribute the wide charge trapping distribution region to the relaxation of the contact line position during the charging process that accompanies the contact angle relaxation (Figure 4.1b and c). As it is shown in Figure 4.1c, the TPCL recedes for $\sim 200 \mu \mathrm{m}$ during charging process and the slowly receding contact line leaves behind a trace of charges on the surface, which eventually forms the observed charge trapping rim. The calculations shown in Figure 4.6 also indicate the well-known fact that local electric field diverges near the TPCL and is therefore much higher than anywhere at along the electrode/fluoropolymer interface. As a consequence, we assume that charge injection from the bottom electrode is negligible compared to the TPCL region.

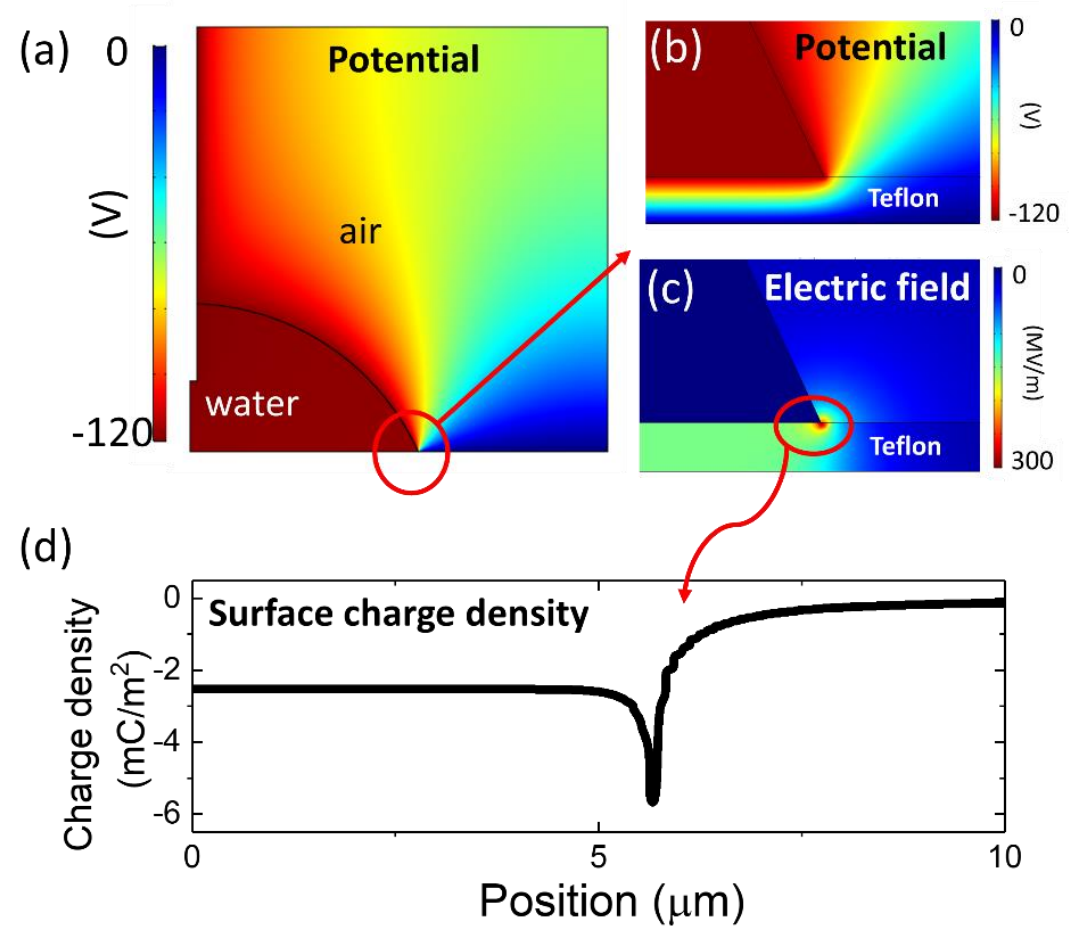

Figure 4.6. Fringe effect simulation using finite element method (on Comsol platform)(a) of the potential of the whole simulation region, (b) the potential, (c) the electric field and (d) the charge density on the drop near the contact line. For details of the simulations, see S.I. and Figure S4.8. 
In order to reduce the width of the deposited rim of charges, we suppress the geometric relaxation of the drop during charging by confining it between two parallel plates at a distance of $h=100 \mu \mathrm{m}$ (Figure 4.7a and 4.7b). $h$ is simply achieved by placing a $100 \mu \mathrm{m}$ thick glass space between the two plates. The lower surface is a Tefloncoated substrate as before and the upper one is an ITO coated glass that serves as an electrode during the charging process. These two surfaces confine a drop during charging and reduce the displacement of the contact line $\Delta R$ for the same amount of contact angle relaxation $\Delta \theta$ as in Figure 4.1, with $\Delta R \propto h \Delta(\cos \theta)$. KPFM measurements after removing the top surface and the drop demonstrate that indeed a much narrower rim of charges is deposited with a width of about $20 \mu \mathrm{m}$, as shown in Figure 4.7. The average surface potential within the rim is $-10 \mathrm{~V}$, corresponding to the trapped charge density $\sigma_{T}=-0.22 \mathrm{mC} / \mathrm{m}^{2}$. From these results, we could also conclude that a much smaller but finite charge density is deposited at the solid-liquid interface away from the contact line, as seen in Figure 4.4. However, the edge of the charged rim is still sharp, suggesting that a further reduction of the width of the rim towards the intrinsic limit should be possible. Thus, by altering the charging voltage, charging time and controlling the TPCL motion, we demonstrated that EWCI is a promising strategy to produce surface charge distributions with microscale resolution.

There are other approaches to manipulate the TPCL than shown in this work, to meet the requirements of the application at hand. For example, charge injection with a straight boundary can be achieved by dipping the sample partially into a container filled with water resulting in a straight TPCL. It is also conceivable to write a line of surface charge by dragging a small drop or liquid meniscus attached to a microcapillary across a solid surface while applying a voltage, similar to the previous report by Banpurkar et $\mathrm{al}^{[59]}$. If the applied voltage is varied depending on the position on the surface, EWCI should thus enable the writing of arbitrary charge patterns with a wide range of possible applications (see Figure S4.9).

The charge densities generated by EWCI are comparable (or even slightly higher) to conventional charge injection methods such as corona discharge or electron beam injection. While the lateral resolution of electron beam (or even AFM-based) charge injection is higher ${ }^{[60]}$, EWCI allows for reasonably sharp charge patterns on the micrometer scale with minimal equipment requirements. Compared to corona discharge, EWCI is clearly superior both regarding the ability of patterning and regarding the stability of the injected charge, in particular in the presence of humidity. 
(a)
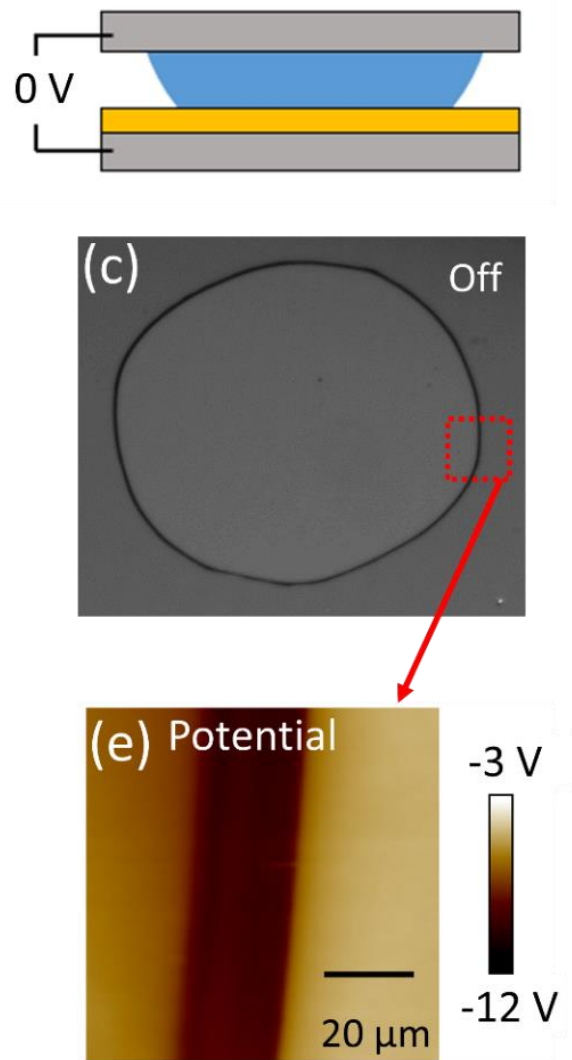

(b)
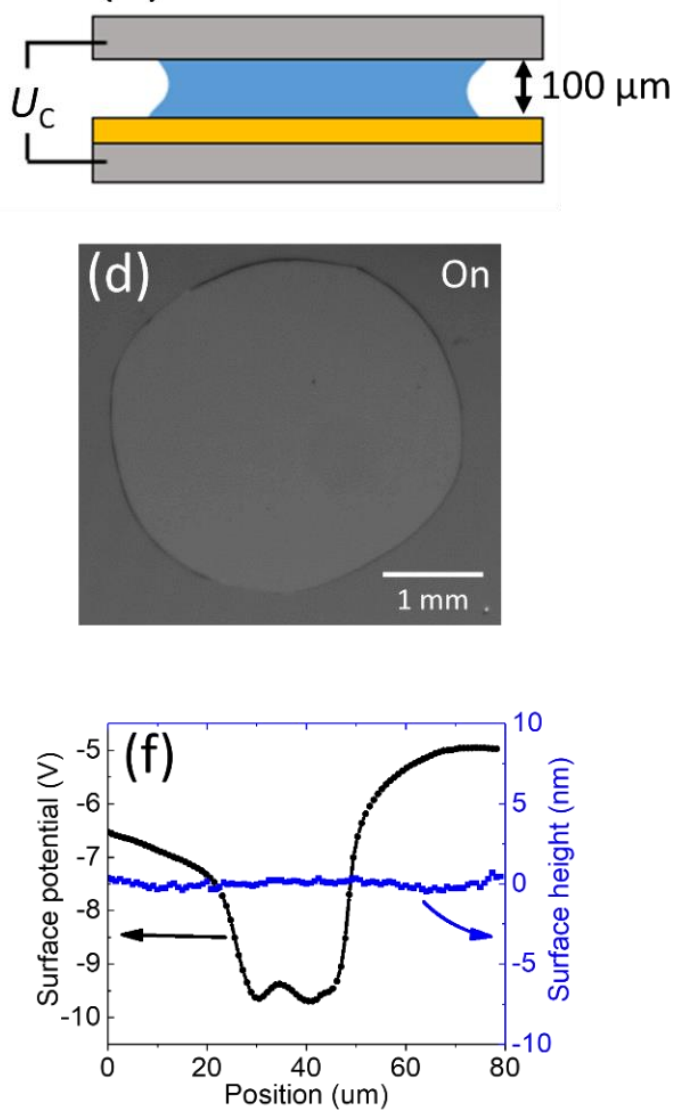

Figure 4.7. Generation of narrow charge patterns using a confined drop with $U_{C}=-90 \mathrm{~V}$ for 5 min. (a) and (b) schematic setup in voltage off and on state. The parts in blue, orange and gray are water, Teflon AF and ITO layer, respectively. The thickness of Teflon AF layer is $800 \mathrm{~nm}$. (c) and (d) corresponding bottom views in transmission illustrating the small variation in radius. (e) KPFM surface potential map of the region indicated in (c) after tuning off the voltage and removing the water. (f) line profiles of surface topography and surface potential of charged region in (e).

\subsection{Conclusion}

In this work, we conclusively show that, the deposition of surface charges from an aqueous drop on an electrically insulating fluoropolymer surface in an EW configuration at high voltage, indeed preferentially occur along the contact line of the 
drop, in accordance with the established EW theory but deviating from recent suggestions based on unconventional and/or unstable EW systems ${ }^{[27,36-37]}$ in which the maximum charge densities are reported accumulated in the center of the drop. This observation is confirmed by a combination of three experimental techniques, namely local contact angle at zero voltage, the asymmetry of the EW response, and KPFM.

Furthermore, we propose this EW-assisted charge injection (EWCI) method as a simple and low-cost strategy for generating surface charges at microscale. We demonstrate the tunability of the charge density and the charged regions by simply adjusting the electric charging conditions or confining the motion of TPCL. No vacuum process or other complex facilities are needed. In addition, negative charge patterns are very stable over long times, in dry and humid environment and even in water. We believe this proposed EWCI strategy will be beneficial for a wide range of research and applications which require controllable surface charges.

\subsection{Experimental Section}

\subsubsection{Preparation of Teflon films}

ITO/glass substrates are cleaned in a Liquid Crystal Display (LCD) cleaning line for G2.5 glass $(400 \mathrm{~mm} \times 500 \mathrm{~mm}$ ). Subsequently, $800 \mathrm{~nm}$ thick AFP films are prepared by screen printing Teflon AF 1600 solution with solvent of FC-43 (The Chemours Company, USA), followed by baking on a hot plate at $95{ }^{\circ} \mathrm{C}$ for $1 \mathrm{~min}$ to remove residual solvent and additional baking in an oven at $185^{\circ} \mathrm{C}$ for $30 \mathrm{~min}$ to anneal the film. All processes are carried out in a clean room. More details on the fabrication process can be found in Ref. ${ }^{[61]}$.

\subsubsection{Surface charging}

Teflon surfaces were charged by applying DC voltages $U_{\mathrm{c}}$ of up to $\pm 140 \mathrm{~V}$ for 2 to 15 min between the ITO electrodes on the substrate (kept at electrical ground potential) and a platinum $(\mathrm{Pt})$ wire $(0.1 \mathrm{~mm}$ diameter $)$ immerse into a $5 \mu \mathrm{L}$ drop of de-ionized water (MilliQ). Charging the surface is performed at room temperature $\left(\sim 25^{\circ} \mathrm{C}\right)$ in a closed container filled with vapour-saturated air. Electrical voltages are generated by a function waveform generator (33220A, Agilent, USA) in combination with an amplifier (PZD 700, Trek, USA). The charging voltage $U_{c}$ is typically chosen within 
the range of contact angle saturation, in which $\theta$ depends only weakly on the applied voltage (see Figure S4.1). $U_{c}$ is limited to ensure that the simultaneously measured current on the substrate remained below $1 \mu \mathrm{A}$ for all experiments. Since the current induced by the trapping charges is sub-nA level, the leakage current does not indicate the amount of trapped charges. (detailed explanation can be found in the description of Figure S4.6)

\subsubsection{Surface characterization}

\section{Contact angle measurements}

The wettability of the samples is measured using a commercial contact angle goniometer (OCA-15+, Data Physics, Germany). Advancing and receding contact angles of the Teflon surfaces in air prior to charging were $\theta_{a}^{\text {air }}=120^{\circ}$ and $\theta_{r}^{\text {air }}=$ $115^{\circ}$. All subsequent contact angle measurements after charging with and without electrowetting (EW) are carried out in ambient silicone oil (317667, Sigma-Aldrich, USA) with probe droplets of 0.3-0.5 $\mu \mathrm{L}$, substantially smaller than the charging drop. All reported voltages are measured with respect to the grounded ITO electrodes. The water drop is in contact with the Pt wire at all time; i.e. contact angles at zero voltage corresponding to a configuration with an electrically grounded droplet (i.e. the droplet is not freely floating). The advancing and receding contact angles under these conditions are close to $170^{\circ}$ with negligible hysteresis. The EW response is probed by applying a triangular waveform $( \pm 30 \mathrm{~V})$ with a period of $60 \mathrm{~s}$ to the probe drop. The maximum voltage during the EW-surface characterization measurements is kept deliberately low to ensure that the system displays a parabolic response following the Equation 4.2. In Equation 4.2, the capacitance per area is $c=\frac{\varepsilon_{0} \varepsilon_{r}}{d}=2.2 \times$ $10^{-5} \mathrm{~F} / \mathrm{m}^{2} \cdot \gamma=41 \mathrm{~mJ} / \mathrm{m}^{2}$ is the oil-water interfacial tension. $\left(\varepsilon_{0} \varepsilon_{r}\right.$ and $d \approx$ $800 \mathrm{~nm}$ : dielectric permittivity and thickness of Teflon AF layer). The surface charge densities are calculated with Equation 4.3 under the assumption that the deposited charge with density $\sigma_{T}$ resides on top of the Teflon surface and does not penetrate substantially into the bulk of the material. Penetration to a depth $\delta$ would lead to an enhanced capacitance $c(\delta)=c \cdot d /(d-\delta)$. However, as long as $\delta$ is only a few nm, i.e. small fraction of $d$, the resulting correction would thus be minor. In addition, a finite charge on the surface automatically implies that $\theta(U=0)<\theta_{Y}$. Correspondingly, there is a finite screening charge density $\sigma_{D}(U=0)=\sigma_{T} \frac{c_{E D L}}{c+c_{E D L}} \approx$ 
$\sigma_{T}$ on the drop ( $c_{E D L} \gg c$ : electric double layer capacitance). In contrast, we denote $\theta_{Y}=\theta\left(U_{T}\right)$ as Young's angle, which corresponds to the contact angle of zero charge on the drop. For more detailed aspects of EW measurements including their interpretation in the presence or absence of surface charges, see Ref. ${ }^{[6]}$.

Kelvin probe force microscopy (KPFM)

To characterize the electrostatic potential of the surface in more detail and with high lateral resolution, KPFM measurements are performed using a commercial atomic force microscopy (AFM; Dimension Icon Bruker, USA) with conductive (Sb(n)doped $\mathrm{Si}$ ) rectangular tips with a nominal tip radius of $25 \mathrm{~nm}$ (SCM-PIT-V2, BRUKER, USA). Upon applying an AC voltage $\left(U_{A C}=500 \mathrm{mV}, f=60 \sim 62 \mathrm{kHz}\right.$, ) superimpose onto a DC voltage $\left(U_{D C}\right)$ to the AFM tip, the electrostatic force $\left(\mathrm{F}_{\mathrm{es}}\right)$ between the AFM tip and sample is given by:

$$
F_{e l}=\frac{1}{2} \frac{\partial C(z)}{\partial z}\left(U_{D C}-U_{T}+U_{A C} \sin \omega t\right)^{2}
$$

Here $\partial \mathrm{C}(\mathrm{z}) / \partial \mathrm{z}$ is the gradient of the capacitance between tip and sample surface and $U_{T}$ is the trapping voltage. Splitting the force according to their frequency $(\omega)$, we obtain the static $\left(F_{\mathrm{DC}}\right)$ and dynamic $\left(F_{\omega}\right.$ and $\left.F_{2 \omega}\right)$ contributions, as usual,

$$
\begin{gathered}
F_{D C}=\frac{\partial C(z)}{\partial z}\left[\frac{1}{2}\left(U_{D C}-U_{T}\right)^{2}+\frac{1}{4} U_{A C}^{2}\right] \\
F_{\omega}=\frac{\partial C(z)}{\partial z}\left(U_{D C}-U_{T}\right) U_{A C} \sin \omega t \\
F_{2 \omega}=-\frac{1}{4} \frac{\partial C(z)}{\partial z} U_{A C} \cos 2 \omega t
\end{gathered}
$$

The amplitude of $F_{\omega}$ is proportional to $U_{D C}-U_{T}$. To obtain the $U_{T}$ in amplitude modulation KPFM, $U_{D C}$ is adjusted such that $F_{\omega}$ becomes minimal. For a system with a perfectly homogeneous dielectric film and a bottom electrode layer, the surface potential $U_{S}$ is expected to be identical with the trapping voltage $U_{T}$. More details on measuring $U_{T}$ with the KPFM can be found in S.I. and Figure S4.4. 


\section{Acknowledgements}

This work was supported by National Key R\&D Program of China (2016YFB0401501), National Natural Science Foundation of China (Grant No. U1501244, U1601651), Program for Chang Jiang Scholars and Innovative Research Teams in Universities (No. IRT_17R40), Science and Technology Project of Shenzhen Municipal Science and Technology Innovation Committee (GQYCZZ20150721150406), Guangdong Provincial Key Laboratory of Optical Information Materials and Technology (No. 2017B030301007), Science and Technology Program of Guangzhou (No. 2019050001). MOE International Laboratory for Optical Information Technologies and the 111 Project.

\section{Contributions}

Hao $\mathrm{Wu}$, Guofu Zhou and Frieder Mugele conceived the project. Hao Wu designed and performed the experiment. Igor Siretanu, Frieder Mugele and Guofu Zhou supervised this work. Ranabir Dey did the simulation. Dirk van den Ende, Igor Siretanu, Lingling Shui, Guofu Zhou and Frieder Mugele provided suggestions and guidance during this work. Hao $\mathrm{Wu}$ analyzed the experimental data. Dirk van den Ende did the calculation on KPFM measurement method. Hao Wu and Frieder Mugele wrote the first draft. All authors revised the manuscript.

\section{References}

[1] M. I. B. Utama, H. Kleemann, W. Zhao, C. S. Ong, H. Felipe, D. Y. Qiu, H. Cai, H. Li, R. Kou, S. Zhao, Nat. Electron. 2019, 2, 60.

[2] H. Cheema, J. H. Delcamp, Adv. Energy Mater. 2019, 9, 1900162.

[3] H. Wu, L. Wu, X. Zhou, B. Liu, B. Zheng, Small 2018, 14, 1802128.

[4] H. H. Choi, K. Cho, C. D. Frisbie, H. Sirringhaus, V. Podzorov, Nat. Mater. 2017, 17, 2.

[5] H. Wu, R. A. Hayes, Responsive Polymer Surfaces: Dynamics in Surface Topography 2017.

[6] F. Mugele, J. Heikenfeld, Electrowetting: Fundamental Principles and Practical Applications, John Wiley \& Sons, 2018.

[7] D. Baratian, R. Dey, H. Hoek, D. Van Den Ende, F. Mugele, Phys. Rev. Lett. 2018, 120, 214502. 
[8] Y. B. Sawane, S. B. Ogale, A. G. Banpurkar, ACS Appl. Mater. Interfaces 2016, 8, 24049.

[9] C. E. Clement, S. K. Thio, S.-Y. Park, Sens. Actuators, B 2017, 240, 909.

[10] F. Mugele, J.-C. Baret, J. Phys.: Condens. Matter 2005, 17, R705.

[11] H. Wu, R. A. Hayes, F. Li, A. Henzen, L. Shui, G. Zhou, Displays 2018, 53, 47.

[12] Y. Suzuki, D. Miki, M. Edamoto, M. Honzumi, J. Micromech. Microeng. 2010, 20, 104002.

[13] H. Li, Z. Guo, S. Kuang, H. Wang, Y. Wang, T. Wu, Z. L. Wang, G. Zhu, Nano Energy 2019, 103913.

[14] S. Gong, J. Zhang, C. Wang, K. Ren, Z. L. Wang, Adv. Funct. Mater. 2019, 1807618.

[15] M. G. Pollack, R. B. Fair, A. D. Shenderov, Appl. Phys. Lett. 2000, 77, 1725.

[16] S. K. Cho, H. J. Moon, C. J. Kim, J. Microelectromech. Syst. 2003, 12, 70.

[17] B. Berge, J. Peseux, Eur. Phys. J. E: Soft Matter Biol. Phys. 2000, 3, 159.

[18] K. Mishra, D. van den Ende, F. Mugele, Micromachines 2016, 7.

[19] R. A. Hayes, B. J. Feenstra, Nature 2003, 425, 383.

[20] H. Wu, L. Shui, F. Li, R. Hayes, A. Henzen, F. Mugele, G. Zhou, ACS Appl. Nano Mater. 2019, DOI: 10.1021/acsanm.8b02303.

[21] T. Krupenkin, J. A. Taylor, Nature communications 2011, 2, 448.

[22] H. Wu, H. Li, A. Umar, Y. Wang, G. Zhou, Materials 2018, 11, 2474.

[23] M. Mibus, X. Hu, C. Knospe, M. L. Reed, G. Zangari, ACS Appl. Mater. Interfaces 2016, 8, 15767.

[24] B. Raj, M. Dhindsa, N. R. Smith, R. Laughlin, J. Heikenfeld, Langmuir 2009, $25,12387$.

[25] A. Schultz, S. Chevalliot, S. Kuiper, J. Heikenfeld, Thin Solid Films 2013, $534,348$.

[26] J. Cao, Q. An, Z. Liu, M. Jin, Z. Yan, W. Lin, L. Chen, P. Li, X. Wang, G. Zhou, Sens. Actuators, B 2019, 291, 470.

[27] M. Mibus, G. Zangari, ACS Appl. Mater. Interfaces 2017, 9, 42278.

[28] H. J. J. Verheijen, M. W. J. Prins, Langmuir 1999, 15, 6616.

[29] D. Thomas, M. C. Audry, R. M. Thibaut, P. Kleimann, F. Chassagneux, M. Maillard, A. Brioude, Thin Solid Films 2015, 590, 224.

[30] S. Berry, J. Kedzierski, B. Abedian, Langmuir 2007, 23, 12429.

[31] A. G. Banpurkar, Y. Sawane, S. M. Wadhai, C. U. Murade, I. Siretanu, D. van den Ende, F. Mugele, Faraday Discuss. 2017, 199, 29.

[32] M. Vallet, M. Vallade, B. Berge, Eur. Phys. J. B 1999, 11, 583. 
[33] K. Adamiak, Microfluid. Nanofluid. 2006, 2, 471.

[34] J. Buehrle, S. Herminghaus, F. Mugele, Phys. Rev. Lett. 2003, 91.

[35] F. Mugele, J. Buehrle, J. Phys.: Condens. Matter 2007, 19, 375112.

[36] X. M. Li, H. M. Tian, J. Y. Shao, Y. C. Ding, X. L. Chen, L. Wang, B. H. Lu, Adv. Funct. Mater. 2016, 26, 2994.

[37] M. Mibus, X. Y. Hu, C. Knospe, M. L. Reed, G. Zangari, ACS Appl. Mater. Interfaces 2016, 8, 15767.

[38] J. Zhong, Q. Zhong, G. Chen, B. Hu, S. Zhao, X. Li, N. Wu, W. Li, H. Yu, J. Zhou, Energy Environ. Sci. 2016, 9, 3085.

[39] L. Chen, Q. Shi, Y. Sun, T. Nguyen, C. Lee, S. Soh, Adv. Mater. 2018, 30, 1802405.

[40] X. Gao, A. Omosebi, J. Landon, K. Liu, Energy Environ. Sci. 2015, 8, 897.

[41] J. Wang, C. Wu, Y. Dai, Z. Zhao, A. Wang, T. Zhang, Z. L. Wang, Nature communications 2017, 8, 88 .

[42] Q. Sun, D. Wang, Y. Li, J. Zhang, S. Ye, J. Cui, L. Chen, Z. Wang, H.-J. Butt, D. Vollmer, Nat. Mater. 2019, 1.

[43] G. Zhu, Y. Su, P. Bai, J. Chen, Q. Jing, W. Yang, Z. L. Wang, ACS nano 2014, $8,6031$.

[44] J. Chen, J. Yang, Z. Li, X. Fan, Y. Zi, Q. Jing, H. Guo, Z. Wen, K. C. Pradel, S. Niu, ACS nano 2015, 9, 3324.

[45] M. Yu, D. Lin, H. Feng, Y. Zeng, Y. Tong, X. Lu, Angew. Chem., Int. Ed. 2017, 56, 5454.

[46] M. Yang, Y. Zhong, J. Ren, X. Zhou, J. Wei, Z. Zhou, Adv. Energy Mater. 2015, 5, 1500550.

[47] D. Stein, M. Kruithof, C. Dekker, Phys. Rev. Lett. 2004, 93, 035901.

[48] A. M. Bannunah, D. Vllasaliu, J. Lord, S. Stolnik, Mol. Pharmaceutics 2014, $11,4363$.

[49] X. Gao, S. Porada, A. Omosebi, K.-L. Liu, P. Biesheuvel, J. Landon, Water Res. 2016, 92, 275.

[50] X. Zhu, D. Jańczewski, S. Guo, S. S. C. Lee, F. J. Parra Velandia, S. L.-M. Teo, T. He, S. R. Puniredd, G. J. Vancso, ACS Appl. Mater. Interfaces 2014, 7,852 .

[51] M. Aramesh, O. Shimoni, K. Ostrikov, S. Prawer, J. Cervenka, Nanoscale 2015, 7, 5726.

[52] N. Agmon, H. J. Bakker, R. K. Campen, R. H. Henchman, P. Pohl, S. Roke, M. Thämer, A. Hassanali, Chemical Reviews 2016, 116, 7642. 
[53] R. Zimmermann, N. Rein, C. Werner, Physical Chemistry Chemical Physics 2009, 11, 4360 .

[54] J. Lützenkirchen, T. Preočanin, N. Kallay, Phys. Chem. Chem. Phys. 2008, 10, 4946.

[55] R. Zimmermann, U. Freudenberg, R. Schweiß, D. Küttner, C. Werner, Curr. Opin. Colloid Interface Sci. 2010, 15, 196.

[56] T. Preočanin, A. Selmani, P. Lindqvist-Reis, F. Heberling, N. Kallay, J. Lützenkirchen, Colloids Surf., A 2012, 412, 120.

[57] M. Scharnberg, S. Rehders, Ö. Adiyaman, S. Schröder, T. Strunskus, F. Faupel, Org. Electron. 2019, 70, 167.

[58] E. Yilmaz, H. Sezen, S. Suzer, Angew. Chem., Int. Ed. 2012, 51, 5488.

[59] D. 't Mannetje, A. Banpurkar, H. Koppelman, M. H. Duits, D. van den Ende, F. Mugele, Langmuir 2013, 29, 9944.

[60] P. Mesquida, A. Stemmer, Adv. Mater. 2001, 13, 1395.

[61] H. Wu, B. Tang, R. A. Hayes, Y. Dou, Y. Guo, H. Jiang, G. Zhou, Materials 2016, 9, 707.

\section{Supporting information}

\section{Electrowetting response}

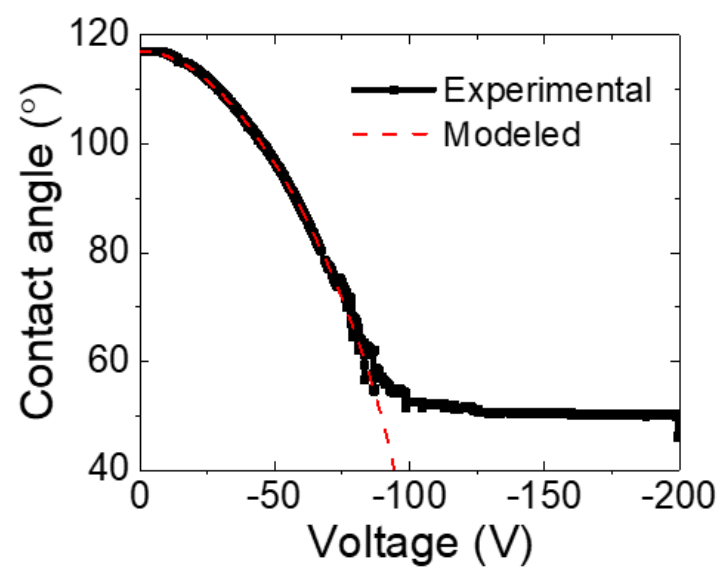

Figure S4.1. Contact angle depending on applied voltage in EWOD. Modeled curve is calculated with Young-Lippmann equation. The dielectric and hydrophobic layer is Teflon AF with a thickness of $\sim 800 \mathrm{~nm}$. 


\section{Charging Teflon film in humid environment}
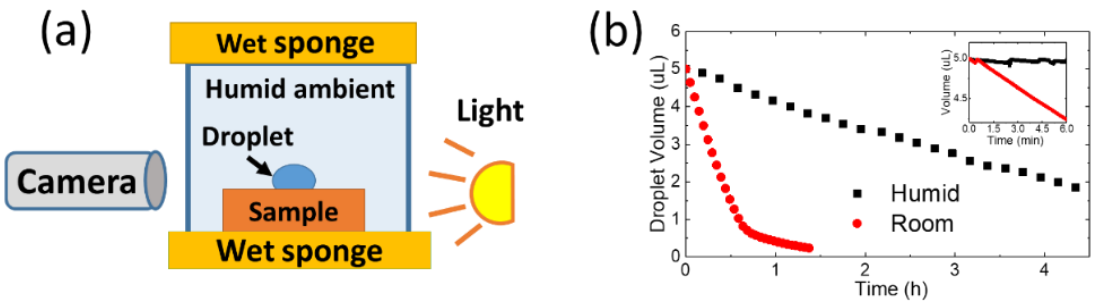

Figure S4.2. (a) Schematic drawing of humid chamber setup. (b) comparison of water droplet evaporation rate in room environment and in a humid chamber.

Schematic drawing of local contact angle measurement by probe-droplet

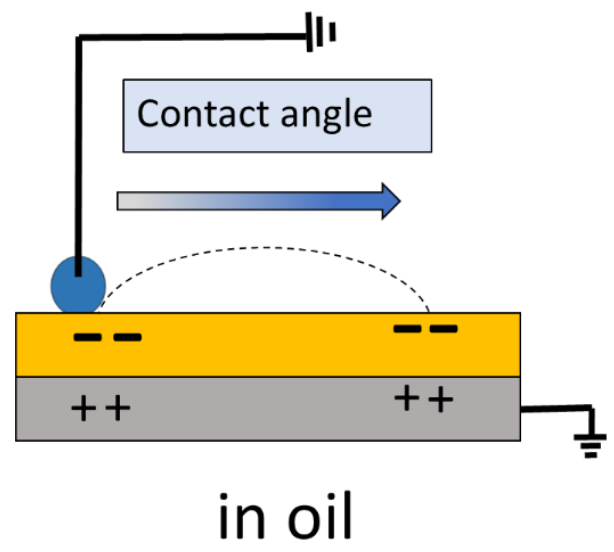

Figure S3. Schematic drawing of local contact angle measurement by probe-droplet. 


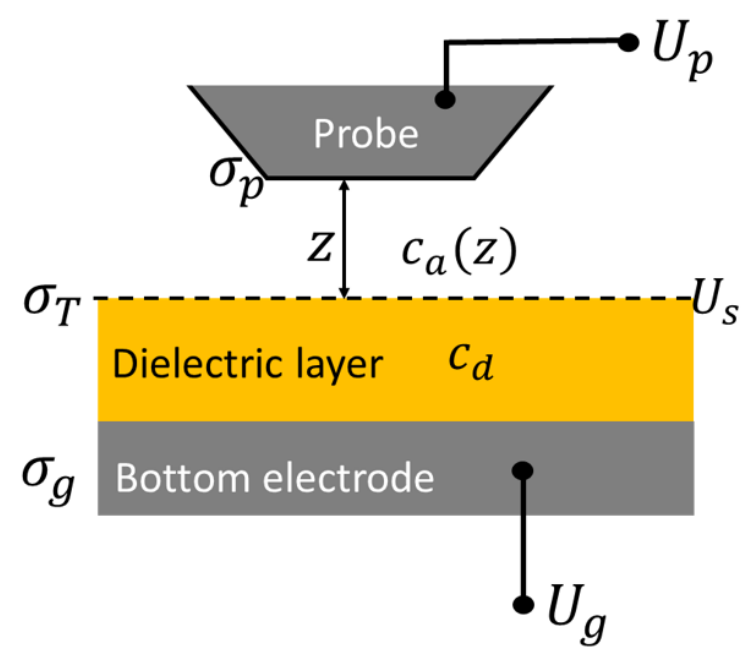

Figure S4.4. The schematic of measuring the trapping voltage $\left(\mathrm{U}_{\mathrm{T}}\right)$ and trapping charge density $\sigma_{\mathrm{T}}$ by utilizing KPFM. A detailed explanation is given below.

According to Figure S4.3 the charge density on the probe of $\operatorname{KPFM}\left(\sigma_{p}\right)$ and on the bottom electrode $\left(\sigma_{g}\right)$ are

$$
\begin{gathered}
\sigma_{p}=c_{a}(z)\left(U_{p}-U_{s}\right) \\
\sigma_{g}=c_{d}\left(U_{g}-U_{s}\right)
\end{gathered}
$$

where the $c_{a}(z)$ is the capacitance per unit area of the air capacitor formed between the probe and the dielectric surface. $c_{a}(z)$ depends on the distance between the probe and the dielectric layer $(\mathrm{z})$, and can be calculated by $c_{a}(z)=\varepsilon_{0} / z \cdot c_{d}$ is the capacitance per unit area of the dielectric layer. $U_{p}, U_{s}$ and $U_{g}$ are the potential at the probe, at the dielectric surface and the bottom electrode. Given the total charge in the system is zero, we get

$$
\sigma_{g}+\sigma_{p}+\sigma_{T}=0
$$

where $\sigma_{T}$ is the trapping charge density on the surface of dielectric layer.

From Equations S4.1 - S4.3, we get: 


$$
\begin{gathered}
U_{s}-U_{p}=\frac{\sigma_{T}}{c_{a}(z)+c_{d}}-\frac{c_{d}}{c+c_{d}}\left(U_{p}-U_{g}\right) \\
U_{s}-U_{g}=\frac{\sigma_{T}}{c_{a}(z)+c_{d}}+\frac{c_{a}}{c+c_{d}}\left(U_{p}-U_{g}\right)
\end{gathered}
$$

Here, we should notice that the surface potential of the dielectric layer

$$
U_{s}=\frac{1}{c_{a}(z)+c_{d}}\left(\sigma_{T}+c_{a}(z) U_{p}+c_{d} U_{g}\right)
$$

is not constant during the measurement. The dielectric surface potential $U_{s}\left(z, U_{p}\right)$ does not only depend on the trapping charge density $\sigma_{T}$, but also on $\mathrm{z}$ and $U_{p}$. We define the trapping voltage as $U_{T}=\sigma_{T} / c_{d}$. Only if the probe is very far away from the dielectric surface, the surface potential equals the trapping voltage, $U_{S}=U_{T}=\sigma_{T} /$ $c_{d}$. The electric energy of the system $W_{e l}$ contain two parts: the energy from the capacitance $W_{c}$ and the energy from the source (battery) $W_{s}$, can be written as:

$$
W_{e l}=W_{c}+W_{s}
$$

The energy of the capacitance contains the energy in the air capacitance between the probe and the surface $\left(W_{a}\right)$ and the energy in the dielectric layer $W_{d}$, and can be calculated as:

$$
\begin{aligned}
W_{c}=W_{a} & +W_{d}=\frac{1}{2} A c_{a}(z)\left(U_{p}-U_{s}\right)^{2}+\frac{1}{2} A c_{d}\left(U_{s}-U_{g}\right)^{2} \\
& =\frac{1}{2} A\left[\frac{\sigma_{T}^{2}}{c_{a}(z)+c_{d}}+\frac{c_{a}(z) c_{d}}{c_{a}(z)+c_{d}}\left(U_{p}-U_{g}\right)^{2}\right]
\end{aligned}
$$

The energy from the source is:

$$
W_{s}=W_{0}-\sigma_{p} A\left(U_{p}-U_{g}\right)
$$

Where $W_{0}$ is the initial energy stored in the source. $A$ is the overlapping area of the probe and the dielectric surface. According to Equations S4.1, S4.2 and S4.3, the charge density on the probe is :

$$
\sigma_{p}=c_{a}(z)\left(\frac{-\sigma_{T}}{c_{a}(z)+c_{d}}+\frac{c_{d}}{c_{a}(z)+c_{d}}\left(U_{p}-U_{g}\right)\right)
$$

Substitute Equation S4.10 to Equation S4.9, we get 


$$
\begin{aligned}
& W_{s}=W_{0}-A c_{a}\left[\frac{-\sigma_{T}}{c_{a}+c_{d}}+\frac{c_{d}}{c_{a}+c_{d}}\left(U_{p}-U_{g}\right)\right]\left(U_{p}-U_{g}\right) \\
= & W_{0}+A \frac{c_{a} \sigma_{T}}{c_{a}+c_{d}}\left(U_{p}-U_{g}\right)-A \frac{c_{a} c_{d}}{c_{a}+c_{d}}\left(U_{p}-U_{g}\right)^{2}
\end{aligned}
$$

Substitute Equations S4.8 and Equations S4.11 to Equations S4.7, the electric energy in the system is

$$
\begin{gathered}
W_{e l}=\frac{1}{2} A\left[\frac{\sigma_{T}{ }^{2}}{c_{a}+c_{d}}+\frac{c_{a} c_{d}}{c_{a}+c_{d}}\left(U_{p}-U_{g}\right)^{2}\right]+W_{0}+A \frac{c_{a} \sigma_{T}}{c_{a}+c_{d}}\left(U_{p}-U_{g}\right) \\
-\frac{c_{a} c_{d}}{c_{a}+c_{d}}\left(U_{p}-U_{g}\right)^{2} \\
=W_{0}+\frac{1}{2} A\left(\frac{\sigma_{T}{ }^{2}}{c_{a}+c_{d}}\right)-\frac{1}{2} A \frac{c_{a} c_{d}}{c_{a}+c_{d}}\left(U_{p}-U_{g}\right)^{2}+A \frac{c_{a} \sigma_{T}}{c_{a}+c_{d}}\left(U_{p}-U_{g}\right)
\end{gathered}
$$

Given the trapping voltage $U_{T}=\sigma_{T} / c_{d}$ and the total capacitance $C(z)$ of the probe and the dielectric layer:

$$
C(z)=A \cdot \frac{c_{a}(z) c_{d}}{c_{a}(z)+c_{d}}
$$

the electrical energy is given by:

$$
W_{e l}=W_{0}-\frac{1}{2} C(z)\left(U_{p}-U_{g}-U_{T}\right)^{2}+\frac{1}{2} A c_{d} U_{T}^{2}
$$

The electric force on the AFM probe is given by the gradient of the energy:

$$
F_{e l}=-\frac{\partial W_{e l}}{\partial z}=\frac{1}{2} \frac{\partial C(z)}{\partial z}\left(U_{p}-U_{g}-U_{T}\right)^{2}
$$

Because $\frac{\partial C}{\partial z}$ is negative, this force is attractive. Since the potential on the tip is the sum of an AC voltage $\left(U_{A C} \sin \omega t\right)$ and a DC voltage $\left(U_{D C}\right), U_{p}$ can be written as:

$$
U_{p}=U_{D C}+U_{A C} \sin \omega t
$$

while the bottom electrode is grounded: $U_{g}=0$. Thus, the electric force is:

$$
F_{e l}=\frac{1}{2} \frac{\partial C(z)}{\partial z}\left(U_{D C}-U_{T}+U_{A C} \sin \omega t\right)^{2}
$$




$$
=\frac{1}{2} \frac{\partial C(z)}{\partial z}\left[\left(U_{D C}-U_{T}\right)^{2}+2\left(U_{D C}-U_{T}\right) U_{A C} \sin \omega t+\frac{1}{2} U_{A C}(1-\cos 2 \omega t)\right]
$$

Splitting the force according to its frequency components, we obtain the static $\left(F_{\mathrm{DC}}\right)$ and dynamic $\left(F_{\omega}\right.$ and $\left.F_{2 \omega}\right)$ contributions:

$$
\begin{gathered}
F_{D C}=\frac{\partial C(z)}{\partial z}\left[\frac{1}{2}\left(U_{D C}-U_{T}\right)^{2}+\frac{1}{4} U_{A C}{ }^{2}\right] \\
F_{\omega}=\frac{\partial C(z)}{\partial z}\left(U_{D C}-U_{T}\right) U_{A C} \sin \omega t \\
F_{2 \omega}=-\frac{1}{4} \frac{\partial C(z)}{\partial z} U_{A C} \cos 2 \omega t
\end{gathered}
$$




\section{Charge trapping contorted by charging voltage and time}
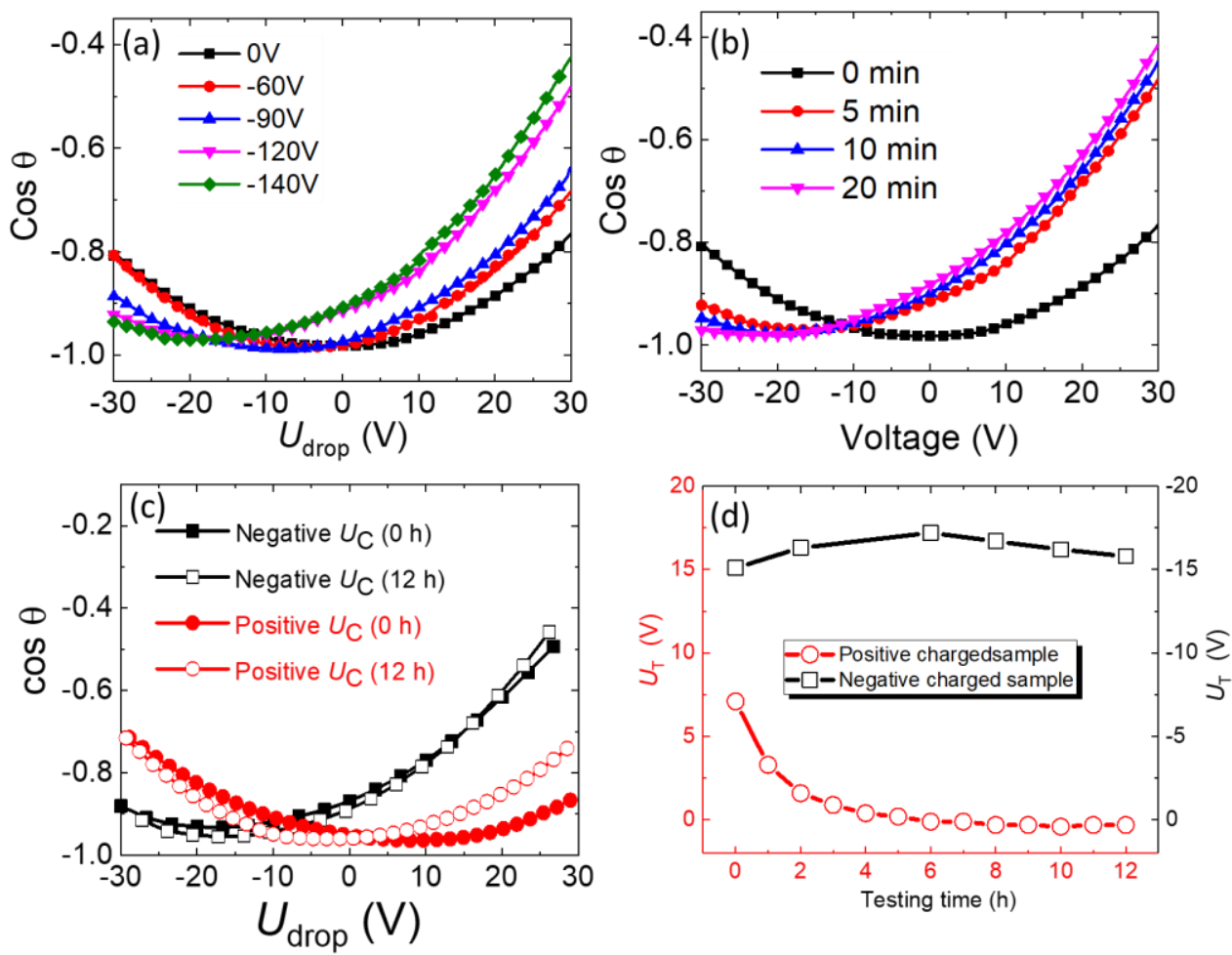

Figure S4. 5. The electrowetting curve of samples charged at (a) various voltage for 5 min and (b) $-120 \mathrm{~V}$ for various time. Insets in (a) and (b) are the trapping voltage dependence of the charging voltage and charging time. (c) Electrowetting response comparison of two charged samples under -120 and $+120 \mathrm{~V}$, respectively, for $5 \mathrm{~min}$. (d) Trapping voltage varying with time driven by a triangular waveform $(30 \mathrm{~V}$ amplitude and $60 \mathrm{~s}$ period). 


\section{Leakage current measurement}

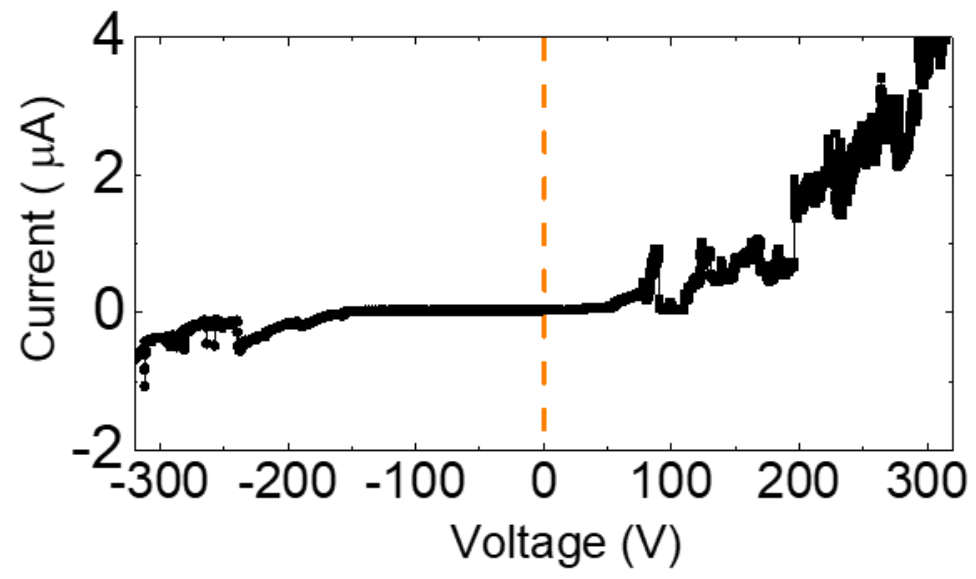

Figure S4. 6. Leakage current depending on applied voltage of sample with a $0.8 \mu \mathrm{m}$ thick Teflon films. 
Charge trapping detected after being treated with water vapor
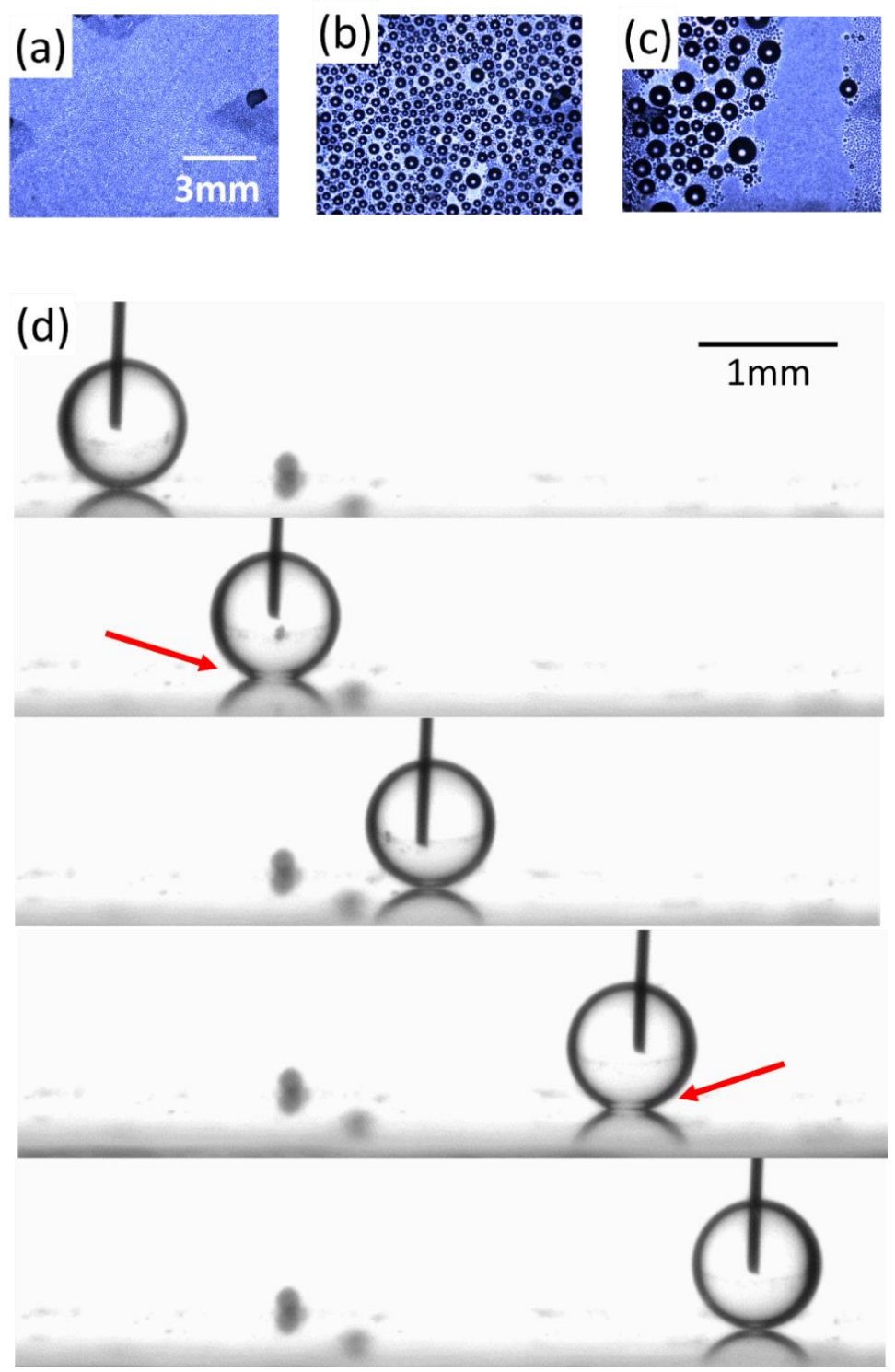

Figure S4.7. (a-c) The image of condensation phenomenon during charge sample (charged by $-120 \mathrm{~V}$ for $5 \mathrm{~min}$ ) being treated with water vapor for $3 \mathrm{~h}$. The sample is vertical placed in a 
humid chamber with water vapor. The water vapor forms (a) small droplets, then (b) the droplets grow bigger, and (c) slide down and now droplet forms on the sample surfaces. (d) Snapshots of local contact angle merriment at different position on the surface of the sample treated by water vapor for $3 \mathrm{~h}$. The red arrows point the TPL region with trapping charges.

\section{Information of fringe effect simulation}
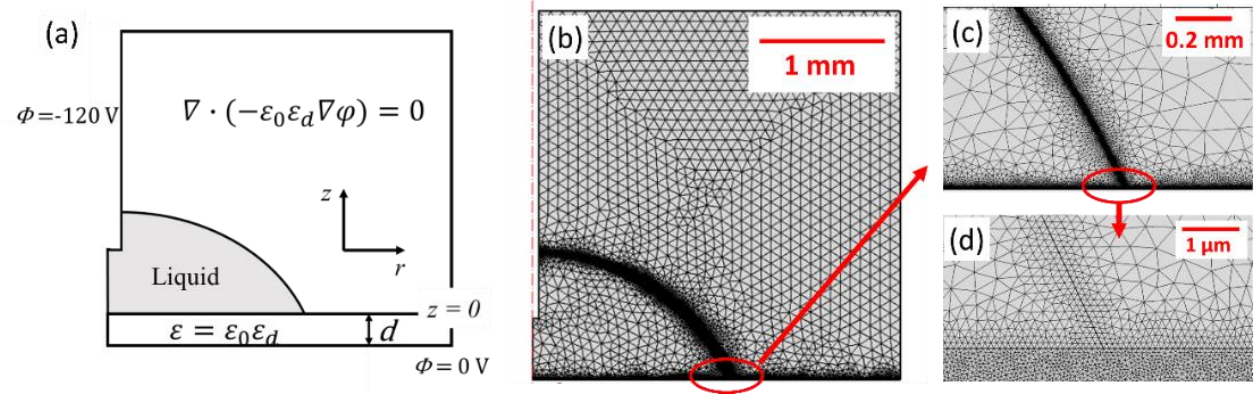

Figure S4.8. (a) Schematic showing the axi-symmetric geometry used for the numerical simulations (b-d) Meshes used for the finite element based simulation using the Comsol platform. The simulation box is $3 \mathrm{~mm} \times 3 \mathrm{~mm}$ in size, and the axis of symmetry is represented by the dash-dotted line in (b). The thickness of the dielectric layer is $800 \mathrm{~nm}$. The size of the elements for the dielectric layer is $10 \mathrm{~nm}$ to $100 \mathrm{~nm}$, and both for the droplet and air domains it is $10 \mathrm{~nm}$ to $100 \mu \mathrm{m}$. The droplet is considered perfectly conductive. The two sides of the platinum electrode, dipped into the droplet, are considered to be at $-120 \mathrm{~V}$ (Dirichlet boundary condition), and the electrode underneath the dielectric is considered to be at $0 \mathrm{~V}$ (Dirichelt boundary condition). The outer domain boundaries are maintained at the no flux condition (Neumann boundary condition). The potential and the field distributions are calculated by solving the equation $\nabla \cdot\left(\varepsilon_{0} \varepsilon_{\mathrm{r}} \nabla \varphi\right)=0$ in each of the domains (dielectric, droplet and air) where $\varepsilon_{0}$ is the vacuum permittivity; $\varepsilon_{\mathrm{r}}$ is the relative permittivity of the insulator (1.93 for Teflon in this case), and $\varphi$ is the electric potential. 


\section{Ideas of using EWCI strategy to produce surface charges in various way}

(a)

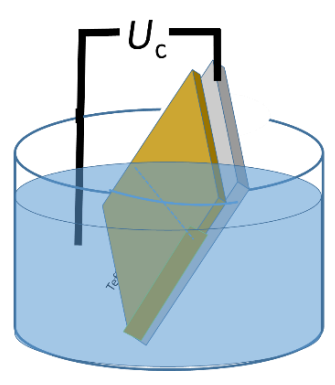

(b)

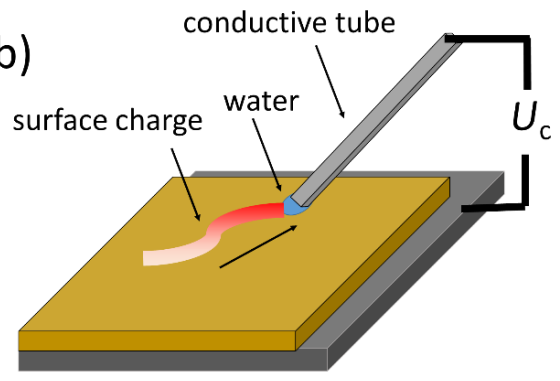

Figure S4.9. (a) Schematic of charge injection at a straight band by putting the samples into a container filled with water. The charge trapping region is supposed to be a straight band because the TPCL is a straight line. (b) Schematic of injection charge on fluoropolymer surfaces with controllable shape and charge density. A small droplet is hold by a conductive tube and moved during the charging process. The charging voltage $\left(\mathrm{U}_{\mathrm{c}}\right)$ can also be tuned during EWCI process as well, in order to deposit surface charge with varying density along the TPCL trace. 


\section{Chapter 5}

\section{Electric power generation from drop impact on charged surfaces*}

In this chapter, we explore the usefulness of the trapped charges. We propose a new type of nanogenerator based on the charge trapping phenomenon, to convert mechanical energy from drops impacting onto a hydrophobic surface into electrical energy. The mechanism of this proposed charge trapping electric nanogenerator (CTENG) is investigated by using a combination of the high speed video microscopy and the current measurements. A physical model is built to quantitatively understand the charge transferring behavior. The scaling law was demonstrated for energy harvesting by using CT-ENG. The electric generation behavior depending on the impact conditions, fluid properties, and external electrical circuitry are investigated to verify the proposed model and to demonstrate the performance of the CT-ENG.

\footnotetext{
* This chapter is based on the preprints: H. Wu, N. Mendel, D. van den Ende, G. Zhou, F. Mugele. Electric generation from drops impacting onto charged surfaces. arXiv:2001.05019 (2020, Submitted)
} 


\subsection{Introduction}

In Chapter 4, we have proposed an EWCI method for surface charge fabrication. When monitoring the leakage current during the EWCI experiment, the author accidently found a very interesting phenomenon: a current signal was generated when the droplet moved together with a conductive probe through the charged region. This finding was very exciting, because a current signal can be easily utilized for energy generation and sensors. A novel Charge Trapping Electric NanoGenerator (CT-ENG) is thus invented after further improving the setup. An application of energy harvesting from water drops utilizing the CT-ENG is demonstrated in this chapter.

In recent years, strategies are proposed for energy harvesting from water motions, such as reverse electrowetting ${ }^{[1-2]}$ and triboelectric nanogenerators ${ }^{[3-8]}$. However, inherent flaws exist in current approaches. Ineluctable external voltage depresses applications of reverse electrowetting. Dependence of low and unstable surface charges on tribo-layers limits the performance of triboelectric nanogenerators (TENG) ${ }^{[9-10]}$. As reported, the droplet energy harvesting efficiency is only $0.01 \%{ }^{[5]}$ by using the conventional TENG. High and stable surface charge density can only be achieved in vacuum environment ${ }^{[11]}$ or by implementing external pumping or excitation sources ${ }^{[10,12]}$. In addition, the surface charges generated by triboelectrification is unpredictable, therefore, the quantitatively understanding of the underlying mechanism of the nanogenerator is challenging.

In this chapter, we propose the CT-ENG and we investigate the electro-mechanical energy conversion by simultaneously measuring the dynamics of drop spreading on a charged substrate and its electrical response with microsecond resolution, both experimentally and theoretically. A physical model is presented, which quantitatively describes the charge transfer mechanism when water drops impact on charged surfaces. The electrical generation behaviors depending on the impact conditions, fluid properties, and external electrical circuitry are investigated to verify the proposed model and to demonstrate the performance of the CT-ENG. 100 days' reliability testing of CT-ENG will also be presented.

\subsection{Results and discussion}

The basic setup of the experiment is sketched in Fig. 5.1a. Millimeter-sized drops of water are released from a syringe a few centimeters above a functionalized and electrically charged substrate. The substrate consists of glass slides coated with a 
transparent conducting ITO layer (to record the spreading area $A(t)$ of the droplet optically) on which a hydrophobic micrometer thin fluoropolymer film is deposited. Prior to use, these substrates have been charged by surface charge printing ${ }^{[15]}$ (SCP, see Suppl. Sect. I) resulting in a trapped surface charge density $\sigma_{t}=-0.12 \mathrm{mC} / \mathrm{m}^{2}$. Approximately $0.5 \mathrm{~mm}$ above the substrate, a thin platinum $(\mathrm{Pt})$ wire has been mounted horizontally to make electrical contact with the droplet that spreads on the substrate (Figure 5.1a). The ITO layer and the Pt wire form the terminals of the nanogenerator, to which a load resistor is connected.

The drop hits the substrate at an impact location in the vicinity of the wire. Upon impacting, the drop first spreads on the surface, forms a pancake-like structure (Figure 5.1c), and then touches the Pt wire while the drop-substrate contact area $A(t)$ reaches its maximum, $A_{\max }$. Subsequently, the drop contracts and finally detaches from the wire. While the drop is initially cylindrically symmetric, its shape gets distorted by the off-axis collision with the wire in later stages of the process. Nevertheless, the contact area $A(t)$ (Figure 5.1d) as extracted from bottom view images, recorded through the transparent substrate (Figure 5.1c, Suppl. Sect IV) follows the typical drop impacting behavior with a characteristic spreading time, $\tau_{h}$, which is of the same order of magnitude as the inertial-capillary time $\tau \equiv\left(\rho r^{3} / \gamma\right)^{1 / 2}$, where $\rho, r$ and $\gamma$ are liquid density, drop radius and interfacial tension, respectively ${ }^{[13-14]}$. We define the hydrodynamic time $\tau_{h}$ as the time between the moment the drop first touches the surface $(t \equiv 0)$ and the moment it reaches maximum spreading (Figure 5.1d). Before the spreading drop touches the Pt wire the simultaneously recorded electrical current remains zero (Figure 5.1c) but jumps abruptly to a large finite value as the drop touches the wire. With a relaxation time of around $2 \mathrm{~ms}$, the current decays initially exponentially. Subsequently, the current changes direction as the drop footprint starts to retract and eventually abruptly vanishes when the drop detaches from the wire. 


\section{a}
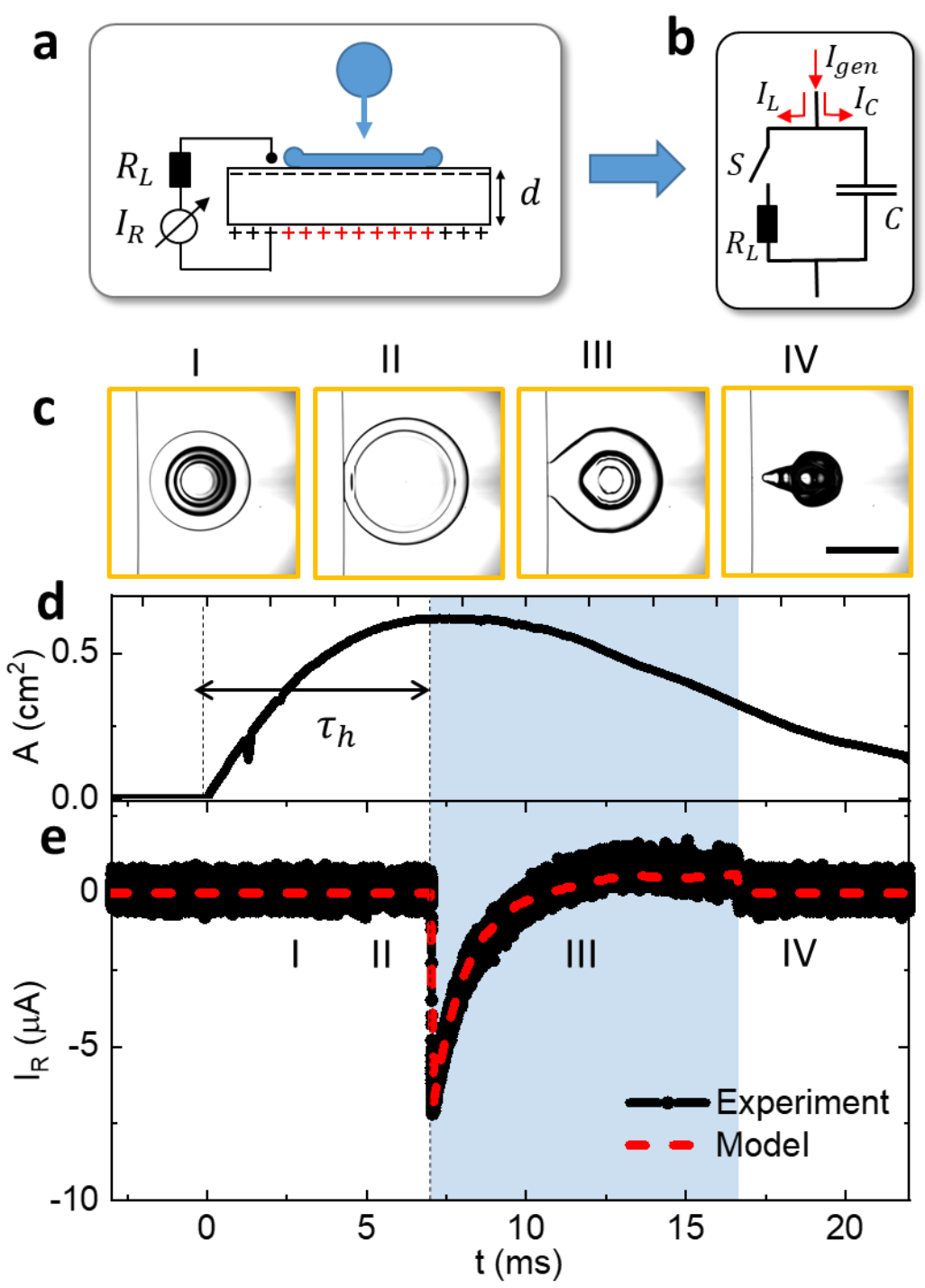

Figure 5.1. Simultaneous characterization of drop impacting dynamics and the electrical responses. a, schematic of a cross section of a drop falling onto negatively charged surface with a top electrode (black '-' symbols), a bottom electrode with positive counter charge, an external load resistor $\mathrm{R}_{\mathrm{L}}$ and current $\mathrm{I}_{\mathrm{R}}$ through the resistor. b, equivalent circuit where the gained charges in the system upon spreading is represented by a generation current $\mathrm{I}_{\text {gen }}$. (see text for details). c, bottom view through transparent substrate of a drop (volume: $33 \mu \mathrm{L}$; liquid: $100 \mathrm{mM} \mathrm{NaCl}$ solution) impacting on a hydrophobic surface (Teflon AF1600): (I) prior to 
contact with wire (top electrode); (II) moment of contacting wire; (III) contracting; (IV) detaching from wire. Scale bar: $5 \mathrm{~mm}$. Simultaneously measured d, drop-solid interfacial area (A) and e, current $\left(\mathrm{I}_{\mathrm{R}}\right)$ of drop impacting on a charged hydrophobic surface $\left(\sigma_{\mathrm{T}}=\right.$ $\left.-0.12 \mathrm{mC} / \mathrm{m}^{2}\right) . \tau_{\mathrm{h}}$ is a hydrodynamic time scale representing the time between drop impact and maximal spreading. Black symbols: experimental data; red curve: model. The current $\mathrm{I}_{\mathrm{R}}$ flows only during drop-wire contact (blue region). The hydrophobic coating is a $800 \mathrm{~nm}$ thick Teflon AF 1600 film (for preparation see Suppl. Sect. I), the load resistor $R_{L}$ is $810 \Omega$.

This characteristic behavior can be understood as follows (see Suppl. Sect. III). As the drop hits the negatively charged surface, the drop initially becomes polarized due to the charges on the solid surface. As long as the drop does not touch the Pt wire, there is no net charge transfer between the two electrodes. At moment the drop touches the wire (Figure 5.1e), part of the countercharge on the bottom electrode (red ' + ' symbols in Figure 5. 1b) flows through the external load resistor $R_{L}$ to the drop, and accumulates in the electric double layer (EDL) at the drop-substrate interface. In the next stage, as the drop contracts, the countercharge flows back to the bottom electrode in pace with the decreasing contact area $A(t)$. Once the drop detaches from the wire, the current abruptly drops to zero (Figure 5.1c-IV).

This charge transfer is driven by the potential difference $U$ between the top and bottom side of the fluoropolymer film that arises from the trapped charge $A(t) \sigma_{t}$ together with the charge $q$ accumulated in the EDL at the liquid-film interface, $Q=$ $\sigma_{t} A(t)+q$ in total, and the countercharge $-Q$ on the ITO electrode at the bottom. Because the thickness of the film is approximately $d \approx 1 \mu \mathrm{m}$ while the Debye length $\lambda_{D}$ is typically a few $n m$, depending on the salt concentration, the capacitance per unit area of the electric double layer $c_{E D L}=O\left(10^{-2} \mathrm{~F} / \mathrm{m}^{2}\right)$ is much larger than the capacitance of the dielectric film $c_{d}=\epsilon_{0} \epsilon_{d} / d \approx 2.2 \times 10^{-5} \mathrm{~F} / \mathrm{m}^{2}\left(\epsilon_{0} \epsilon_{d}\right.$ is the dielectric permittivity of the fluoropolymer layer). Therefore, we neglect the capacity of the EDL can represent the droplet-substrate system as a single capacitance $C \approx$ $c_{d} A(t)$ while the potential over this capacitor is $U=\left(\sigma_{t} A(t)+q\right) / c_{d} A(t)$. Moreover, due to Ohms law, $U=I_{R} R_{L}$, where $I_{R}=-d q / d t$ is the current through the resistor $R_{L}$. Combining both expressions for $U$ we obtain the governing differential equation:

$$
\frac{d q}{d t}+\frac{q(t)}{R_{L} c_{d} A(t)}=\frac{-\sigma_{t}}{R_{L} c_{d}}
$$


This equation describes the charge transfer when the drop makes electrical contact with the Pt wire, so for $t_{o}<t<t_{f}$ where $t_{o}$ is the moment the drop touches the wire and $t_{f}$ the moment the drop detaches from the wire. At $t=t_{o}$ the charge in the EDL $q=0$, while $U=\sigma_{t} / c_{d}$. The sketched process can be represented with an electric circuit as given in Figure 5.1b, where the switch closes at $t=t_{o}$ and opens again at $t=t_{f}$. In the time between, a current $I_{g e n}(t)=\sigma_{t} d A / d t$ is injected in the RC circuit, part of which, $I_{R}=U / \mathrm{R}_{\mathrm{L}}$, flows through the resistor while the other part, $I_{C}=d Q / d t$, flows towards the capacitor, where $Q=\sigma_{t} A(t)+q$. While $I_{g e n}$ scales with $1 / \tau_{h}$, the current $I_{R}$ through the load resistor, which is the device in the applications, scales with the inverse of the electrical relaxation time $\tau_{e l}=R_{L} c_{d} A_{\max }$, where $A_{\max }=A\left(\tau_{h}\right)$ is the maximal value of $A(t)$. Depending on the value of $R_{L}$ and $c_{d}$ both timescales $\left(\tau_{h}\right.$ and $\tau_{e l}$ ) can be quite different (see Suppl. Sect. V for more details).

We consider two cases, 1: the drop touches the Pt wire after spreading ("off-wire" case) or 2: immediately at the moment of impact ("on-wire" case). For the first case when the drop touches the wire at its maximum area $A_{\max }$ (referred as "off-wire", Figure 5.1), the numerically calculated $I_{R}(t)=-d q / d t$ based on Equation 5.1 with $R_{L}=810 \Omega$ and $A(t)$ as measured by the high-speed camera, is shown as the red dashed line in Figure 5.1c. The curve perfectly matches the measured current curve, confirming the proposed mechanism. To explain the process and further verify the model, we first consider the current behavior during a short time directly after impact, $t_{o}<t \ll t_{o}+t_{h}$. During this time the contacting area barely changes, i.e., $A \approx A_{\text {max }}$, and correspondingly, $I_{\text {gen }}=0$. The charge $Q$ at the moment of touching, $t_{0}$, is given by $Q\left(t_{0}\right)=\sigma_{T} A_{\text {max }}$. In this regime Eq. 5.1 reduces to: $d Q / d t+$ $Q(t) /\left(R_{L} c_{d} A_{\max }\right)=0$. With the initial condition just given, the resulting current through $R_{L}$ is $I_{R}=-I_{o} e^{-t / \tau_{e l}}$, where $I_{o}=-\sigma_{t} A_{\max } / \tau_{e l}$ is the initial current at $t=$ $t_{o}$. This exponential decay of the current is characteristic for the discharge of an $R C$ circuit. 

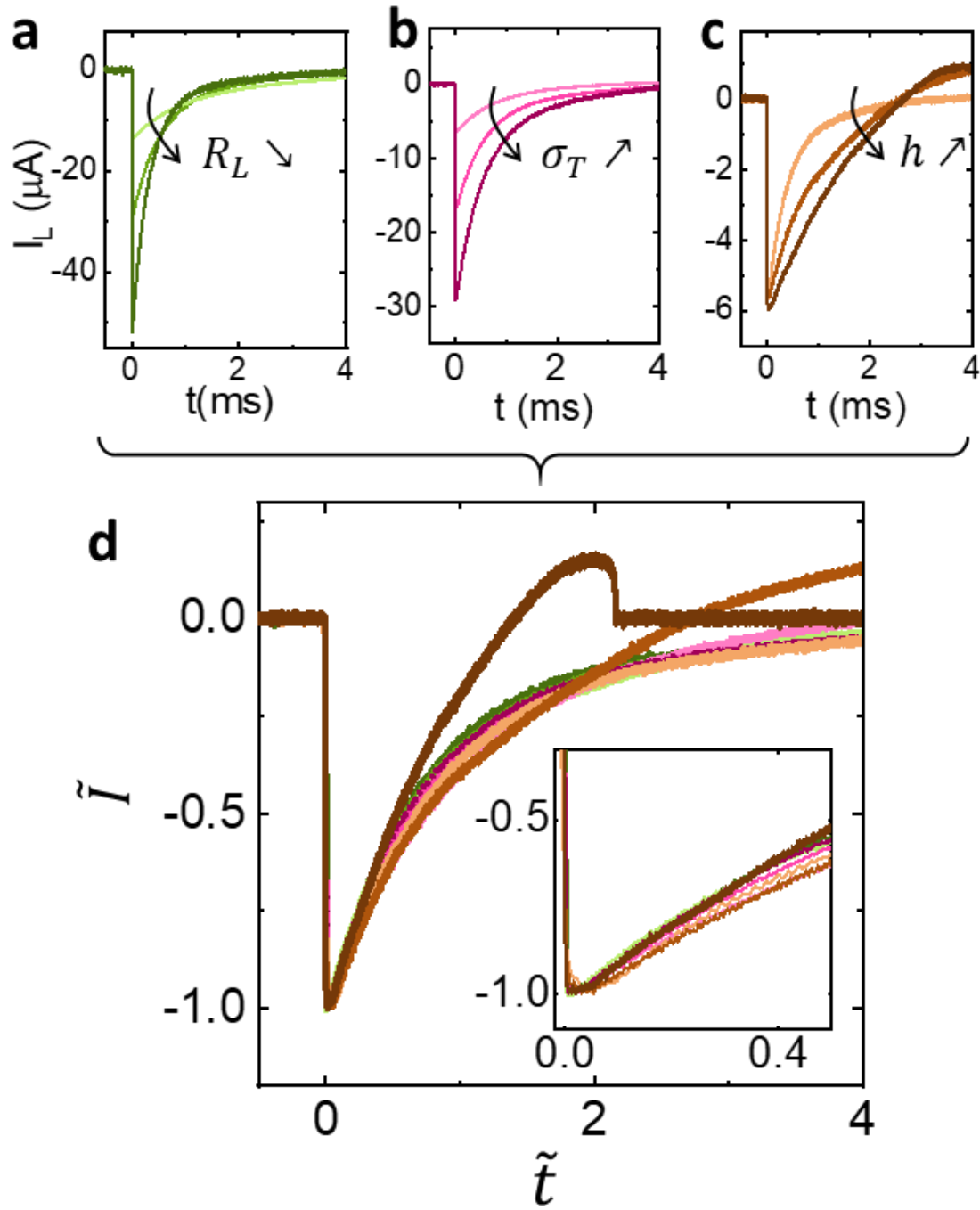

Figure 5.2 Universal current response from impacting drops with varying $R_{L}, \sigma_{T}$ and $h$. Current through load resistor vs. time for "off-wire" impact under various conditions: a, varying $\mathrm{R}_{\mathrm{L}}\left(0.47,0.81,1.65 \mathrm{M} \Omega\right.$ ) fixed $\sigma_{\mathrm{T}}=0.35 \mathrm{mC} / \mathrm{m}^{2} ; \mathrm{h}=43 \mathrm{~mm}$ (oxidized silicon samples); b, varying trapped charge density $\left(\sigma_{\mathrm{T}}=-0.07,-0.20,-0.35 \mathrm{mC} / \mathrm{m}^{2}\right)$; fixed $\mathrm{R}_{\mathrm{L}}=810 \Omega ; \mathrm{h}=43 \mathrm{~mm}$. (oxidized silicon samples) c, varying impact height $(\mathrm{h}=3$, $9,18 \mathrm{~cm}$ ). fixed $\sigma_{\mathrm{T}} \approx-0.1 \mathrm{mC} / \mathrm{m}^{2} ; \mathrm{R}_{\mathrm{L}}=810 \Omega$ (transparent samples); Drop volume for a and $\mathbf{b}$ are $33 \mu \mathrm{L}$, for $\mathbf{c}$ is $17 \mu \mathrm{L}$. $\mathbf{d}$, non-dimensional current response curve vs. non-dimensional time for all data shown in panels a, b, c. For $\mathrm{t} \ll \tau_{\mathrm{h}}$ (inset of $\mathbf{d}$ ), all curves collapse onto the universal response curve of a discharging $\mathrm{RC}$-circuit. 
Current curves obtained from drops impacting on substrates from several heights $h$ (implying variable $A_{\max }$ ) and with different load resistances $R_{L}$, or surface charge densities $\sigma_{T}$ have been plotted in Figure 5.2. After normalizing current and time as $\tilde{I}_{R}=I_{R} / I_{0}$ and $\tilde{t}=t / \tau_{e l}$, respectively, the curves indeed overlap with each other and display the universal discharge behavior as shown in Figure 5.2d. This consistency once more confirms the applicability of the model for the "off-wire" case. Here we used electrowetting-assisted charge injection $(\mathrm{EWCI})^{[15]}$ instead of SCP to achieve stable surface charge densities on a relatively large surface area. The obtained surface charges $\sigma_{T}$ can be determined with an electrowetting (EW) probing method (see Suppl. Sect. II). The calculated $I_{0}$ based on the $\sigma_{t}$ measured by EW, is in good agreement with the measured initial current (see Suppl. Sect. V). Thus, all parameters in the model $\left(R_{L}, c_{d}, \sigma_{t}, A_{\text {max }}, \tau_{h}\right)$ are known and moreover, the measurements fully corroborate the model.

In the second case the drop impacts directly on the Pt wire ("on-wire"). Here we use a low $R_{L}$ value, so $\tau_{e l} \ll \tau_{h}$ and because the charge on the film adjust itself almost immediately, the total current $I_{g e n}(t)$ flows through the resistor. Consequently, $I_{R}=$ $I_{g e n}=\sigma_{T} d A / d t$. The calculated current $I_{R}(t)$ matches again perfectly with the measured current curve, without any adjustable parameter (see Figure 5.3a). According to this model the total charge transferred through $R_{L}$ from drop impact ( $A=0$ ) to maximal spreading $\left(A=A_{\max }\right), Q_{\text {tran }}=q\left(\tau_{h}\right)=\int_{0}^{\tau_{h}} I_{R} d t=$ $\int_{0}^{A_{\text {max }}} \sigma_{T} d A=\sigma_{T} A_{\text {max }}$, is proportional to $A_{\text {max }}$. The measured dependence of $Q_{\text {tran }}$ on $A_{\max }$ for $\tau_{e l} \ll \tau_{h}$ is given in Figure 5.3b and confirms this linear relationship.

From a more general perspective, the roles of $I_{g e n}$ and $I_{C}$ can be revealed by comparing the current response for "on-wire" and "off-wire" impact at low and high load resistance (Figure 5.3c and 3d). For very low $R_{L}$, i.e. $\tau_{e l} \ll \tau_{h}$, "off-wire" impact leads to a very high current peak, $I_{o}=-\sigma_{t} A_{\max } / \tau_{e l}$, as the orange curve in Figure $5.3 \mathrm{c}$ shows. This is caused by the rapid discharge (with a decay time $\tau_{d}=$ $\left.\tau_{e l} A\left(t_{0}\right) / A_{\max }\right)$ of the initial charge $Q\left(t_{0}\right)=\sigma_{T} A\left(t_{0}\right)$. After the initial discharge, $I_{R}$ follows the same response as observed in a "on-wire" situation (blue curve, Figure $5.3 \mathrm{c}$ ), which is governed by the generation current $I_{g e n}$. For higher $R_{L}$, i.e. longer $\tau_{e l}$, the overall current level, in particular the $I_{0}$ for "off-line" case, is much lower compared to the one with low $R_{L}$ (Figure 5.3d), because $I_{o} \propto 1 / \tau_{e l}$. The current responses from "off-wire" and "on-wire" impacts are much more similar because the electrical and the hydrodynamic time scale are no longer separated and hence the 
contributions of $I_{C}$ and $I_{g e n}$ to the current through the load resistor are comparable in magnitude.
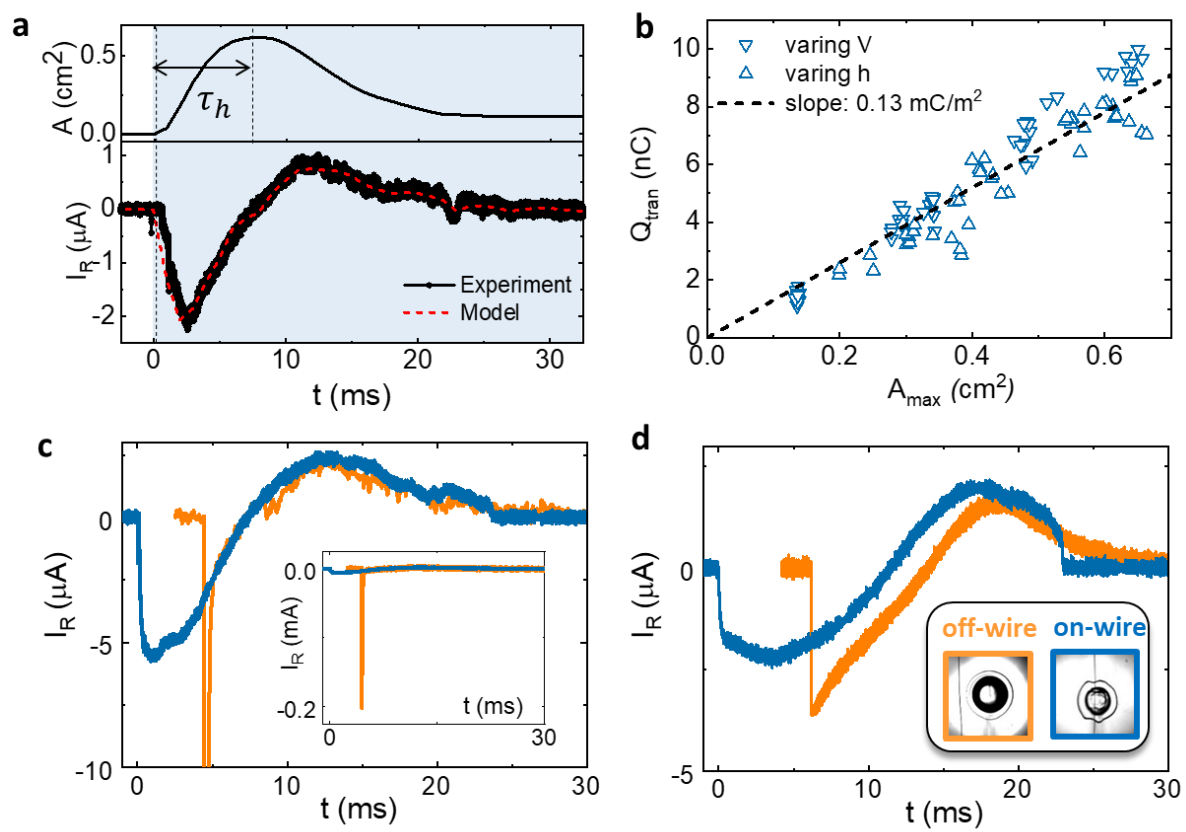

Figure 5.3 Drop impacting "off-wire" vs "on-wire". a, simultaneously measured drop-solid interfacial area $(\mathrm{A})$ and current $\left(\mathrm{I}_{\mathrm{R}}\right)$ of drop impacting on a charged hydrophobic surface $\left(\sigma_{\mathrm{T}}=\right.$ $\left.-0.13 \mathrm{mC} / \mathrm{m}^{2}, c_{d}=2.2 \times 10^{-5} \mathrm{~F} / \mathrm{m}^{2}, R_{L}=0 \mathrm{k} \Omega\right)$. b, amount of transferred charge $\mathrm{q}$ depending on maximum spreading area $A_{\text {max }}$. The slope of the dashed line is the surface charge density $\sigma_{\mathrm{T}}$. (c, d) Current response for on-wire (blue) and off-wire (orange) impact with c, low load resistance $\left(\mathrm{R}_{\mathrm{L}}=100 \mathrm{k} \Omega, c_{d}=1.48 \times 10^{-5} \mathrm{~F} / \mathrm{m}^{2}, \sigma_{\mathrm{T}}=-0.35 \mathrm{mC} / \mathrm{m}^{2}, \tau_{\mathrm{el}} \ll\right.$ $\left.\tau_{h}\right)$ and $\mathbf{d}$, high load resistance $\left(\mathrm{R}_{\mathrm{L}}=6.5 \mathrm{M} \Omega, \tau_{\mathrm{el}} \approx \tau_{\mathrm{h}}\right)$. Note the high peak current in the inset of $\mathbf{c}$ is limited by the internal resistance of the detection electronics. Samples used for $\mathbf{a}$, b are transparent samples charged by SCP method, and for $\mathbf{c}, \mathbf{d}$ are oxidized silicon samples charged by EWCI method.

Because the drop, which impacts on the charged substrate, transmits electrical power $\left(R_{L} I_{R}^{2}\right)$ to the load resistor ${ }^{[16]}$, the setup described above is literally a charge-trappingbased electric nanogenerator (CT-ENG). Depending on the charge injection method, this CT-ENG can be modified towards other types of nanogenerators, such as an electret nanogenerator ${ }^{[11,17]}$, a triboelectric nanogenerator ${ }^{[18-19]}$ or an electret- 
triboelectric nanogenerator ${ }^{[8,20]}$. Here, we study the energy harvesting capabilities of this CT-ENG device from water drops using the proposed physical model. The harvested energy, i.e. the energy dissipated in the load resistor, during the entire process is (see Suppl. Sect. V):

$$
\Delta E=R \int_{\mathrm{t}_{\mathrm{o}}}^{\mathrm{t}_{f}} I_{R}^{2}(t) d t=E_{0} g\left(\tau_{h} / \tau_{e l}, t_{o} / \tau_{h}, t_{f} / \tau_{h},\left\{\alpha_{n}\right\}\right)
$$

where $E_{0}=\sigma_{T}^{2} A_{\max } / c_{d}$. Hence, $\Delta E$ scales linearly with $\sigma_{t}^{2}, A_{\max }$ and $c_{d}^{-1}$. The scaling factor $g$ is just a dimensionless function of $\tau_{h}, \tau_{e l}, t_{o}, t_{f}$, and the coefficients $\left\{\alpha_{n}\right\}$, describing the time evolution of the contact area $A(t) / A_{\text {max }}$. This prediction is confirmed by our experimental results using samples with various surface charge densities, see Fig. 5.4. By plotting the harvested energy as $\Delta E / E_{o}$ versus $\tau_{e l} / \tau_{h}$, we observe in Figure 5.4b a nice collapse of the curves with different $\sigma_{t}$ for both $t_{o}=0$ and $t_{o}=\tau_{h}$, over a wide range of load resistors.

Moreover, it turns out that "off-wire" impact leads to a more efficient energy conversion than "on-wire" impact for $R_{L}<R_{0} \approx 6 M \Omega$ where $R_{0}=\tau_{h} /\left(c_{d} A_{\max }\right)$. This is caused by the initial extremely high "off-wire" current response due to $I_{C}$, which leads to an efficient energy conversion, while the $I_{\text {gen }}$ governed "on-wire" current remains low throughout the entire process. Numerical calculations based on Eqs. 5.1 and 5.2 with a typical $A(t)$ profile as observed in the experiments also indicate that for a relatively small electrical timescale $\tau_{e l}$, i.e. for low $R_{L}$ values, a $t_{o}$ value close to $\tau_{h}$ leads to a more efficient energy conversion (see Fig. S5.9).

To further demonstrate the influence of the water droplet properties on the performance of the CT-ENG, we use water solutions with various conductivities, including rain water from the Enschede area in the Netherlands. In "off-wire" mode, we find that for low conductivities the initial current $I_{0}$ increases with increasing water conductivity, saturating at high conductivities towards $\sigma_{T} / R_{L} c_{d}$, see Figure $5.4 \mathrm{c}$. This can be understood from the additional resistance, $R_{\text {drop }}$ in the droplet due to low conductivity of the water, so we have to modify the initial current expression as $I_{0}=\sigma_{T} /\left(R_{L}+R_{\text {drop }}\right) c_{d}$ (see Suppl. Sect. V). Moreover, as reported previously, for negative charges, the charge injected by EW is very stable, even in a humid or water environment, for more than 100 days, see Figure 5.4d. Consequently, the performance of a CT-ENG device produced by EWCI is unaffected, at least during 100 days. 

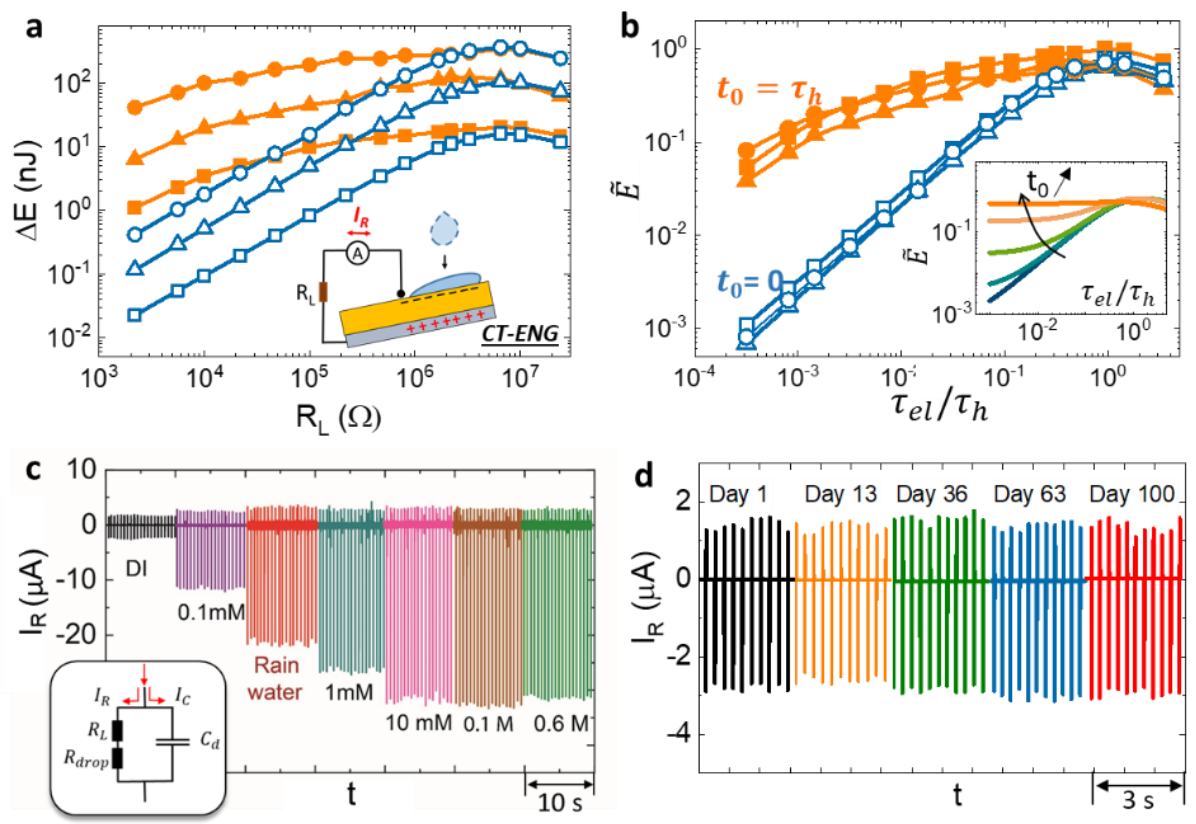

Figure 5.4 Applications of Charge Trapping Electrical Nanogenerator (CT-ENG). a. Harvested energy $\Delta E$ from one water drop (volume: $33 \mu \mathrm{L}$, liquid: $100 \mathrm{mM} \mathrm{NaCl}$ solution) falling from $43 \mathrm{~mm}$ height by using Charge Trapping Electrical Nanogenerator (CT-ENG) with three trapped charge density (squares: $\sigma_{\mathrm{T}}=-0.07 \mathrm{mC} / \mathrm{m}^{2} ;$ triangles: $-0.2 \mathrm{mC} / \mathrm{m}^{2}$; circle: $-0.35 \mathrm{mC} / \mathrm{m}^{2}$ ). Inset is a schematic of CT-ENG. b. normalized harvested energy per drop $\left(\Delta E / E_{0}\right)$ vs. $\tau_{e l} / \tau_{h}$ for on-wire impact (blue) and off-wire impact (orange) with variable trapping charge densities. Inset is a calculated harvested energy $\Delta E / E_{0}$ vs. $\tau_{\mathrm{el}} / \tau_{\mathrm{h}}$ based on a numerical solution of Eqs. 5.1 and 5.2, for impact times $0<\mathrm{t}_{0}<\tau_{\mathrm{h}}$ (see Supplementary Section V). c. The current generated from multiple water drops with different conductivities $\left(R_{L}=810 \mathrm{k} \Omega, c_{d}=1.48 \times 10^{-5} \mathrm{~F} / \mathrm{m}^{2}, \sigma_{\mathrm{T}}=-0.35 \mathrm{mC} / \mathrm{m}^{2}\right)$ Inset is the equivalent circuit. d. Reliability testing of current generated from multiple falling drops for 100 days $\left(R_{L}=\right.$ $\left.6.5 \mathrm{M} \Omega, c_{d}=1.48 \times 10^{-5} \mathrm{~F} / \mathrm{m}^{2}, \sigma_{\mathrm{T}}=-0.27 \mathrm{mC} / \mathrm{m}^{2}\right)$.

\subsection{Sample preparation and solid-liquid area measurement}

Transparent samples used in Figure 5.1, 5.2c, 5.3a and 5.3b are fabricated based on glass substrates in a pilot manufacturing line in a high standard cleanroom (Class 100, ISO 5) in South China Normal University. Glasses substrates coated with indium tin 
oxide (ITO) are cleaned in a Liquid Crystal Display (LCD) cleaning line. $800 \mathrm{~nm}$ thick Teflon AF1600 films (the Chemours Company, USA) are prepared by screen printer (Autech Enterprise Co., Ltd. China). Afterwards, the substrates are baked on a hot plate at $95{ }^{\circ} \mathrm{C}$ for $1 \mathrm{~min}$ and in an oven at $185{ }^{\circ} \mathrm{C}$ for $30 \mathrm{~min}$ to anneal the film. The substrates are cut into $7.5 \mathrm{~cm} \times 2.5 \mathrm{~cm}$ sized rectangular samples. (More details on this fabrication process can be found in Ref. ${ }^{[21]}$ ). Surface charges on these transparent samples are prepared by Surface Charge Printing method (Suppl. Sect. I).

Oxidized silicon samples used in Figure 5.2a, 2b, 3a, 3b and 4 are prepared in the NanoLab in University of Twente (cleanroom Class 100, ISO 5). Czochralski (CZ)grown p-type (100)-oriented silicon wafers are used as bottom electrode. $300 \mathrm{~nm}$ thick $\mathrm{SiO}_{2}$ layer is grown on the doped silicon substrate with a low pressure chemical vapor deposition (LPCVD, Tempress, the Netherlands) at a temperature of $1100{ }^{\circ} \mathrm{C}$ for 4.5 h. The wafer substrates are cut into $3 \mathrm{~cm} \times 2 \mathrm{~cm}$ sized rectangular samples. The samples are cleaned by $99 \% \mathrm{HNO}_{3}$ for 2 min and rinsed by purified water afterwards. A $1 \mu \mathrm{m}$ thick hydrophobic layer is prepared by spin-coating (spinning speed of 1500 rpm) with Teflon AF1600 solution (Chemours, USA). The substrates are heated to 85 ${ }^{\circ} \mathrm{C}$ for $1 \mathrm{~min}$ and following by an annealing process at $185^{\circ} \mathrm{C}$ for $1 \mathrm{~h}$. By utilizing the electrowetting-assisted charge injection (EWCI) method, surface charge densities up to $-0.35 \mathrm{mC} / \mathrm{m}^{2}$ can be generated (see Suppl. Sect. II). The top electrode of a Pt wire (diameter of $0.1 \mathrm{~mm}$ ) is fixed with glue or tape on the surface of both transparent glass and oxidized silicon samples.

The solid-liquid contact area during the drop impact on the surface is extracted from the images recorded with a highspeed camera (Fastcam SA5, Photron, Japan). The details of the image processing are described in Suppl. Sect. IV. The highspeed current measurements are done with a fast trans-impedance amplifier (HF2TA, Zurich Instrument, Switzerland) combining with a digital phosphor oscilloscope (TDS503B, Tektronix, United States). A picoammeter (Model 6487, Keithley, United States) is also used for some current measurements.

\section{Acknowledgements}

This work was supported by National Key R\&D Program of China (2016YFB0401501), National Natural Science Foundation of China (Grant No. 51561135014, U1501244), Program for Chang Jiang Scholars and Innovative Research Teams in Universities (No. IRT_17R40), Science and Technology Program 
of Guangzhou (No. 2019050001), Guangdong Provincial Key Laboratory of Optical Information Materials and Technology (No. 2017B030301007), MOE International Laboratory for Optical Information Technologies and the 111 Project.

\section{Contributions}

Hao $\mathrm{Wu}$ discovered the initial phenomenon, designed the CT-ENG device, figured out its working principle and proposed the numerical calculation. Dirk van den Ende did the non-dimensional calculation. Frieder Mugele suggested the synchronized imaging and current measuring method. Frieder Mugele and Guofu Zhou supervised this work. Hao Wu and Niels Mendel performed the main experiments. Guofu Zhou, Hongwei Jiang and Alex Henzen (and some other engineers from Guofu Zhou's group) produced the transparent Teflon coated samples. Daniel Wijnperle produced the $\mathrm{SiO}_{2}$ coatings. Hao Wu, Niels Mendel, Dirk van den Ende and Frieder Mugele analyzed the experimental data. Guofu Zhou provided suggestions and guidance during this work. Hao Wu wrote this chapter under Frieder Mugele's guidance. Hao Wu and Dirk van den Ende revised this chapter.

\section{References}

[1] T. Krupenkin, J. A. Taylor, Nature communications 2011, 2, 448.

[2] H. Yang, S. Hong, B. Koo, D. Lee, Y.-B. Kim, Nano energy 2017, 31, 450.

[3] Z. H. Lin, G. Cheng, L. Lin, S. Lee, Z. L. Wang, Angewandte Chemie 2013, $125,12777$.

[4] G. Zhu, Y. Su, P. Bai, J. Chen, Q. Jing, W. Yang, Z. L. Wang, ACS nano 2014, 8,6031 .

[5] Z. H. Lin, G. Cheng, S. Lee, K. C. Pradel, Z. L. Wang, Advanced Materials 2014, 26, 4690.

[6] S. S. Kwak, S. Lin, J. H. Lee, H. Ryu, T. Y. Kim, H. Zhong, H. Chen, S.-W. Kim, ACS nano 2016, 10, 7297.

[7] D. Yoo, S.-C. Park, S. Lee, J.-Y. Sim, I. Song, D. Choi, H. Lim, D. S. Kim, Nano Energy 2019, 57, 424.

[8] K. Tao, H. Yi, Y. Yang, H. Chang, J. Wu, L. Tang, Z. Yang, N. Wang, L. Hu, Y. Fu, Nano Energy 2019, 104197. 
[9] H. Zou, Y. Zhang, L. Guo, P. Wang, X. He, G. Dai, H. Zheng, C. Chen, A. C. Wang, C. Xu, Nature communications 2019, 10, 1427.

[10] W. Liu, Z. Wang, G. Wang, G. Liu, J. Chen, X. Pu, Y. Xi, X. Wang, H. Guo, C. Hu, Nature communications 2019, 10, 1426.

[11] K. Zhao, X. Wang, Y. Yang, Advanced Materials Technologies 2017, 2, 1600233.

[12] L. Xu, T. Z. Bu, X. D. Yang, C. Zhang, Z. L. Wang, Nano Energy 2018, 49, 625.

[13] J. C. Bird, R. Dhiman, H.-M. Kwon, K. K. Varanasi, Nature 2013, 503, 385.

[14] D. Richard, C. Clanet, D. Quéré, Nature 2002, 417, 811.

[15] H. Wu, R. Dey, I. Siretanu, D. van den Ende, L. Shui, G. Zhou, F. Mugele, Small 2019.

[16] X. Wang, J. Song, J. Liu, Z. L. Wang, Science 2007, 316, 102.

[17] Y. Suzuki, IEEJ Transactions on Electrical and Electronic Engineering 2011, $6,101$.

[18] Z. L. Wang, T. Jiang, L. Xu, Nano Energy 2017, 39, 9.

[19] W. Tang, T. Jiang, F. R. Fan, A. F. Yu, C. Zhang, X. Cao, Z. L. Wang, Advanced Functional Materials 2015, 25, 3718.

[20] H. Zhang, S. Feng, D. He, Y. Xu, M. Yang, J. Bai, Nano Energy 2018, 48, 256.

[21] H. Wu, B. Tang, R. A. Hayes, Y. Dou, Y. Guo, H. Jiang, G. Zhou, Materials 2016, 9, 707. 


\section{Supporting information}

\section{Charging transparent substrates by surface charge printing}

In order to record the evolution of the drop-substrate contact area, we need high-speed imaging through the bottom of the substrates. Therefore, transparent substrates are required. As reported previously, surface charges can be generated from a water drop impacting on/contacting with hydrophobic surfaces ${ }^{[1-3]}$. For instance, Q. Sun et al. used surface charge printing (SCP) for programmed droplet transport ${ }^{[1]}$. In this work, we use SCP to generate a surface charge distribution on a fluoropolymer surface. The schematic is shown in Fig. S5.1a. A series of droplets with volume of $33 \mu \mathrm{L}$ are released from a height of around $5 \mathrm{~cm}$ and impacts on a fixed spot on the hydrophobic surface. This results in a surface charge at the impact spot. According to our observation, the surface charge increases with every drop impact. The surface charge density reaches a plateau after around 500 drops (Fig. S5.1b). This surface charge saturation phenomenon has also been observed for the case of drop sliding on a hydrophobic perfluoro octadecyltrichlorosilane (PFOTS) surface ${ }^{[3]}$. With the SCP method, surface charges of approximately $0.15 \mathrm{mC} / \mathrm{m}^{2}$ can be generated (depending on drop height and substrate conditions).

a

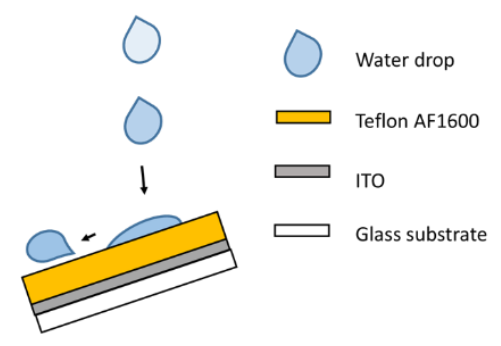

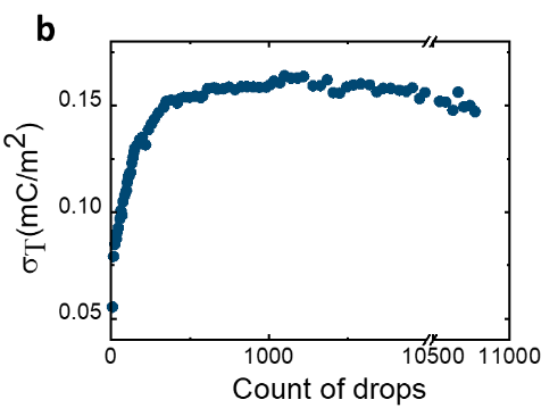

Figure S5.1 a, schematic of surface charge produced by surface charge printing(SCP) method. b, surface charge density depending on the count number of impacting water drops. 


\section{Charging $\mathrm{Si} / \mathrm{SiO}_{2} /$ Teflon substrates by improved electrowetting-assisted charge injection}

In order to verify the proposed physical model and to enhance the performance of the Charge Trapping Electrical Nanogenerator (CT-ENG), substrates with a stable and high surface charge density are required. For this purpose, ElectroWetting-assisted Charge Injection (EWCI) method is applied.
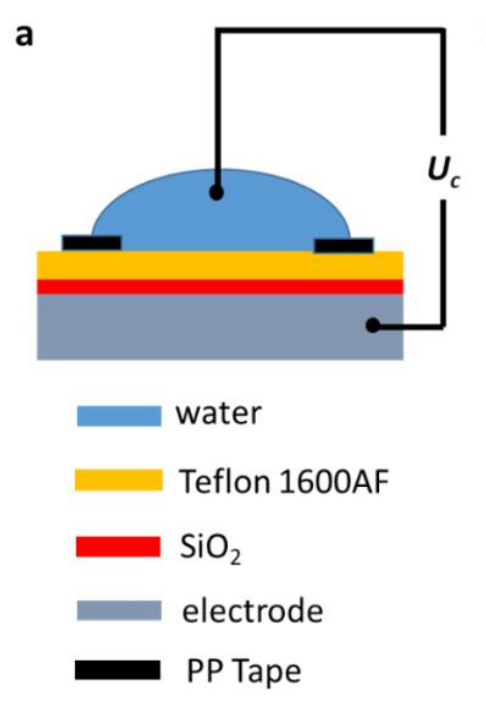
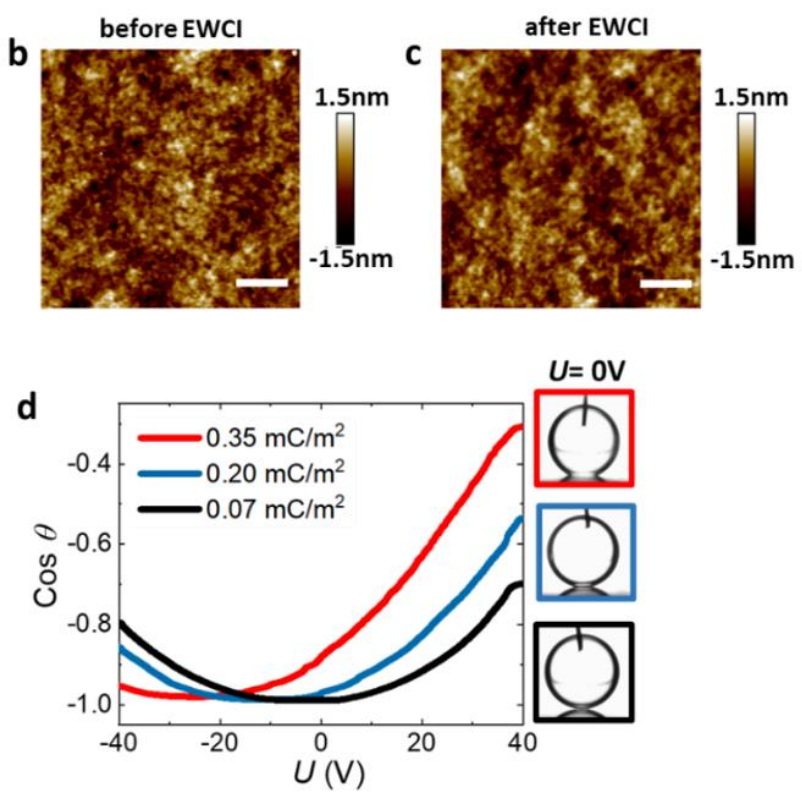

Figure S5.2 a, schematic of the enhanced Electrowetting-assisted Charge Injection (EWCI) process (thickness of $\mathrm{SiO}_{2}: 300 \mathrm{~nm}$; thickness of Teflon AF1600: $1 \mu \mathrm{m}$ ). Atomic force microscopy (AFM) images of surfaces $\mathbf{b}$, before and $\mathbf{c}$, after EWCI process. Scale bar: $200 \mathrm{~nm}$. d, surface charge densities on three charged surfaces measured by Electrowetting probe. Charge injection conditions for these three samples are, red: -400V for $15 \mathrm{~min}$; blue: $-300 \mathrm{~V}$ for $15 \mathrm{~min}$; black: non-charged. Insets are the drop images under applied voltage $U=0 \mathrm{~V}$. The frame color indicates the surface charge density: red for $-0.35 \mathrm{mC} / \mathrm{m}^{2}$, blue for -0.20 $\mathrm{mC} / \mathrm{m}^{2}$ and black for $-0.07 \mathrm{mC} / \mathrm{m}^{2}$.

According to Chapter 4, charges can be injected at the three phase contact line (TPCL) region on fluoropolymer-water interfaces during electrowetting due to the locally enhanced electric field. To deposit a homogeneous charge distribution over the full contact area, a relatively high and more homogeneous electric field should be applied. To this purpose, we protect the substrate near the TPCL region by polypropylene (PP) 
tape and introduce a $300 \mathrm{~nm}$ thin thermally grown $\mathrm{SiO}_{2}$ layer as a dielectric layer underneath the fluoropolymer (Teflon AF1600) layer. The dielectric strength of the thermally grown $\mathrm{SiO}_{2}$ is higher than $1000 \mathrm{~V} / \mu \mathrm{m}$, which is much higher than for Teflon $\operatorname{AF}(20-150 \mathrm{~V} / \mu \mathrm{m})^{[4-5]}$. By simply placing a $300 \mathrm{~nm} \mathrm{SiO}{ }_{2}$ layer underneath the $1 \mu \mathrm{m}$ fluoropolymer film, a potential of $400 \mathrm{~V}$ can be applied to the combined film (using deionized water) without damage. Consequently, a high electric field can be applied over a large arear of the dielectric layer. Fig. S5.2 shows the schematic of this improved EWCI process. It also shows that the surface topography does not change under EWCI, consistent with the results of Chapter 4.After charging the surface in this way, the water is removed from the fluoropolymer surface and the surface charge densities can be tested by electrowetting (EW), as shown in Fig. S5.2d. For a neutral surface, the EW response curve, i.e. $\cos \theta$, where $\theta$ is the contact angle, versus applied voltage $U$, is symmetric around $U=0$. When the hydrophobic surface has been charged, the symmetry axis will be shifted to $U=U_{T}$. From this shift $U_{T}$, the surface charge density can be calculated as $\sigma_{T}=c_{d} U_{T}$, where the $c_{d}$ is the capacitance of the dielectric layer per area ${ }^{[6]}$. For a pristine surface, a spontaneous surface charge density of $-0.07 \mathrm{mC} / \mathrm{m}^{2}$ has been found. By applying $-300 \mathrm{~V}$ or $-400 \mathrm{~V}$ for 15 minutes a charge density of respectively $-0.20 \mathrm{mC} / \mathrm{m}^{2}$ and $-0.35 \mathrm{mC} / \mathrm{m}^{2}$ is achieved.

\section{Charge transfer process}

As shown in Fig. 1, the process of a drop impacting on a solid surface can be divided into 4 stages: I) the drop impacts and spreads on the charged surface; II) the drop reaches its maximum spreading and touches the conductive (Pt) wire; III) the drop contracts; IV) the drop detaches from the wire. Here, we discuss the charge transfer during these stages, using the schematics and the equivalent circuits that are shown in Fig. S5.3.

I) Before the drop touches the wire, all counter charges are at the bottom electrode. Since the switch in the equivalent circuit is open, there flows no current through the load resistor $R_{L}$.

II) Because the capacitance of electric double layer $\left(C_{E D L}\right)$ at the liquid-solid interface is much larger than the dielectric capacitance $\left(C_{D i e l}\right)$, counter charge tends to migrate from the bottom electrode through $R_{L}$ to the liquid-solid interface, when the drop touches the wire. The amount of charge transferred between the two electrodes 
depends on both the hydrodynamic $\tau_{h}$ and electric timescale $\tau_{e l}$ we discussed in the main text. When the resistance is small, $\tau_{e l} \ll \tau_{h}$, and the local counter charge will be transferred to the EDL.

III) After reaching its maximum spreading, the drop starts to contract, and the liquidsolid contact area $A(t)$ decreases. As shown in Fig. S5.3-III, the counter charge flows back to the bottom electrode during this stage, leading to a positive current through $R_{L}$. In the case $\tau_{e l} \ll \tau_{h}$, the current $I_{R}$ is dominated by the drop dynamics, while when $\tau_{e l}$ is comparable or larger than $\tau_{h}, I_{R}$ is determined by both the drop dynamics and the RC circuit response.

IV) When the drop detaches from the wire and bounces off or slides downhill (depending on whether the surface is tilted), the current through the load resistor becomes zero.
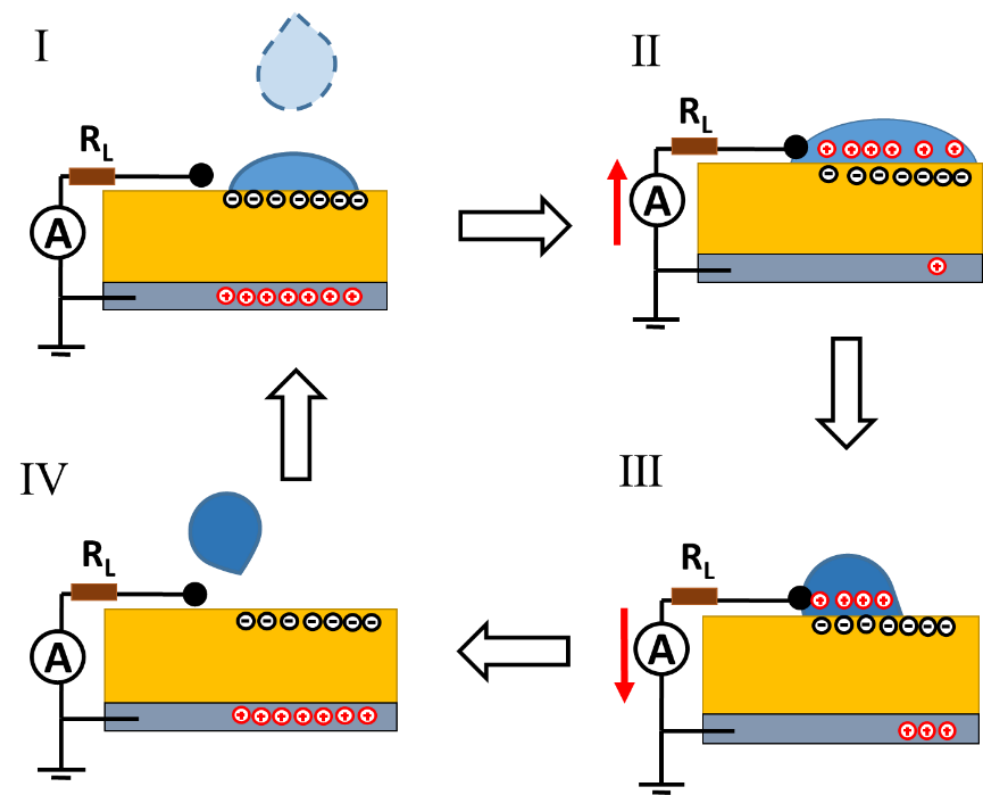

Figure S5.3 Schematic of the four stages of a drop impacting on a charged surface: I) drop impacting and spreading on the charged surface; II) drop reaching its maximum spreading and touching the conductive (Pt) wire; III) drop contracting; IV) drop detaching the wire. 


\section{Extracting the liquid-substrate contact area $A(t)$}

The impacting drop is observed through the substrate using a microscope in reflection mode. Although it is more vividly to observe the drop impact from aside, one can only observe the outer profile of the drop, while the liquid-solid contact area is hard to quantify. To determine the liquid-solid contact area $A(t)$ correctly, we process the images observed in a reflection mode.
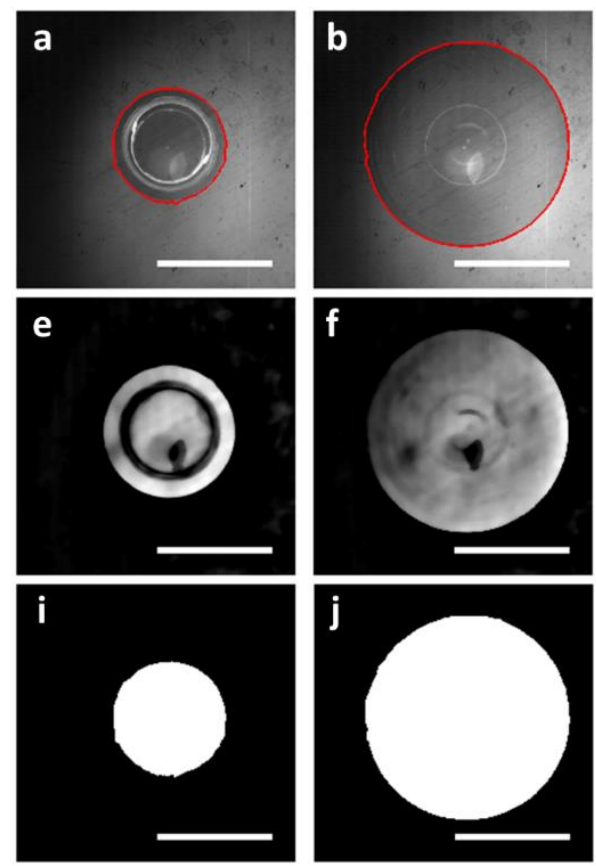
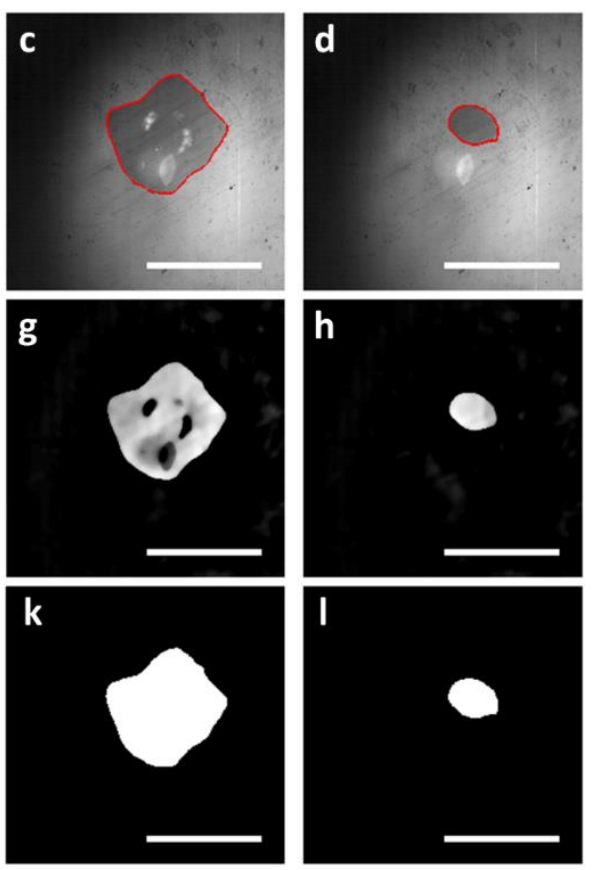

Figure S5.4 a-d, non-processed images (extracted liquid-solid interfacial area is marked in red); e-h, images after background subtraction and median filtering (steps 1 and 2); i-l, binary masks of the extracted liquid-solid interfacial area. Scale bar: $5 \mathrm{~mm}$.

Images are recorded using a high speed camera (Fastcam SA5, Photron, Tokyo, Japan) at 10,000 fps and a microscope (Eclipse Ti, Nikon, Tokyo, Japan). Due to limited contrast with the background, the liquid-solid contact area could not be extracted by simple thresholding techniques (Fig. S5.4a-d). Therefore, we process the images in several steps (using the scikit-image library ${ }^{[7]}$, Fig. S5.4e-1):

1) The background is removed by subtracting a Gaussian blur of the image (kernel: 40px disk), and subtracting the first recorded image in the sequence (with zero liquidsolid interfacial area). Subtracting a Gaussian blur with a large kernel removes the 
background gradient. Subtracting the initial image reduces the noise in the images considerably (e.g. noise from pollution of the backside of the substrate).

2) A median filter (kernel: 10px disk) is applied to the images. Other than by applying a standard Gaussian blur to smoothen the image, a median filter smoothens the image while preserving sharp edges. (Figs. S5.4 e-h).

3) Edges are detected by applying a $3 \times 3$ Sobel filter. The output image is binarized by applying a (global) threshold.

4) Morphological transformations are applied to remove small objects and fill holes from the binary mask(Figs. S4i-1). The number of pixels on the resulting mask is counted and converted using a predetermined scaling factor.

\section{Modeling the electric behavior}

The governing equation for the charge flowing through the load resistor is given by Eq. 5.1 :

$$
-\frac{d q}{d t}=I(t)=\frac{U(t)}{R_{L}}=\frac{\sigma_{t} A(t)+q(t)}{R_{L} c_{d} A(t)}
$$

Where $t_{0}<t<t_{f}$ and $q\left(t_{0}\right)=0$. This equation can be rewritten as:

$$
\frac{d q}{d t}+\frac{q(t)}{R_{L} c_{d} A(t)}=\frac{-\sigma_{t}}{R_{L} c_{d}}
$$

Defining $x=q / q_{0}, s=t / \tau_{h}, f(s)=A\left(\tau_{h} s\right) / A_{\max }, q_{0}=-\sigma_{t} A_{\max }, \tau_{e l}=$ $R_{L} c_{d} A_{\text {max }}$ and $\beta=\tau_{h} / \tau_{e l}$, we make Eq. S5.2 dimensionless:

$$
\frac{d x}{d s}+\beta \frac{x(s)}{f(s)}=\beta
$$

By inspection we observe that the solution of Eq. S5.3, with boundary condition $x\left(s_{0}\right)=0$, is given by:

$$
x\left(s, \beta, s_{o}\right)=\beta \int_{s_{0}}^{s} \mathrm{e}^{-\beta L\left(s, s_{1}\right)} d s_{1}
$$


where $L\left(s, s_{1}\right)=\int_{s_{1}}^{s}\left[f\left(s_{2}\right)\right]^{-1} d s_{2}$ and $s_{0}<s_{1}<s_{2}<s$. Note that $\partial L / \partial s=$ $1 / f(s)$. Eq. S5.4 can be solved numerically, once one has an expression or parametrization for $f(s)$.

From Eq. S5.3 we observe that for $s=s_{0}$ the derivative is given by $d x / d s=\beta$ unless $f\left(s_{0}\right)=0$. In that case we obtain for the derivative, using l'Hopital's rule: $d x / d s=\beta f^{\prime}\left(s_{0}\right) /\left(f^{\prime}\left(s_{0}\right)+\beta\right)$, where $f^{\prime}(s)$ is the derivative of $f(s)$.

In dimensional form we obtain for the charge $q$ and current $I=-d q / d t$ as a function of time $t$ :

$$
q(t)=-\sigma_{t} A_{\max } x(s), \quad I(t)=\frac{\sigma_{t} A_{\max }}{\tau_{h}}\left(\frac{d x}{d s}\right)
$$

for $t / \tau_{h}=s>s_{o}=t_{o} / \tau_{h}$. Thus for $t=t_{0}$ we get in case $A\left(t_{0}\right) \neq 0$ (off-wire):

$$
I_{0}=\frac{\sigma_{t} A_{\max }}{\tau_{e l}}=\frac{\sigma_{t}}{R_{L} c_{d}}
$$

and in case $A\left(t_{0}\right)=0$ (on-wire):

$$
I_{0}=\frac{\sigma_{t} A^{\prime}\left(t_{0}\right)}{1+R_{L} c_{d} A^{\prime}\left(t_{0}\right)} \simeq \frac{\sigma_{t}}{R_{L} c_{d}+\tau_{h} / A_{\max }}
$$

Here we use the notation $A^{\prime}(t)=d A / d t$. At impact we estimate $A^{\prime}(0) \approx A_{\max } / \tau_{h}$. Therefor, the off-wire current can be much larger than the on-wire current. The excess current decays exponentially with a short decay time because at $t=t_{0}$ :

$$
\left(\frac{d^{2} x}{d s^{2}}\right)_{s_{0}}=\frac{-\beta}{f\left(s_{0}\right)}\left(\frac{d x}{d s}\right)_{s_{o}}
$$

So the initial slope of $I(t)$ is given by $(d I / d t)_{t_{0}}=-I_{0} / \tau_{e l}$.

If the process starts at $s_{0}=1$, where $d f / d s=0$, we can approximate Eq. 3 for $1<$ $s \ll 2$ as:

$$
\frac{d x}{d s}+\beta x(s)=\beta
$$

Which has as solution $x(s)=1-e^{-\beta\left(s-s_{0}\right)}$ and $d x / d s=\beta e^{-\beta\left(s-s_{0}\right)}$. In dimensional form last equation reads: 


$$
I(t)=\frac{\sigma_{t} A_{\max }}{\tau_{e l}} e^{-\left(t-t_{0}\right) / \tau_{e l}}
$$

The total harvested energy is given by:

$$
\Delta E=R_{L} \int_{t_{0}}^{\infty} I^{2} d t=\beta^{-1} \frac{\sigma_{t}^{2} A_{\max }}{c_{d}} \int_{s_{0}}^{s_{f}}\left(\frac{d x}{d s}\right)^{2} d s
$$

Because the integral

$$
\frac{1}{\beta} \int_{s_{0}}^{s_{f}}\left(\frac{d x}{d s}\right)^{2} d s=F\left(\beta, s_{0}, s_{f},\left\{\alpha_{n}\right\}\right)
$$

is just a function of $\beta=\tau_{h} / \tau_{e l}, s_{0}, s_{f}$ and $\left\{\alpha_{n}\right\}$, where the parameters $\left\{\alpha_{n}\right\}$ describe the time evolution of $A(t) / A_{\max }$, we can write the harvested energy as:

$$
\Delta E=\frac{\sigma_{t}^{2} A_{\max }}{c_{d}} g\left(\beta, s_{0}, s_{f},\left\{\alpha_{n}\right\}\right)
$$

We can tune $\beta$ and $s_{0}$ such that $\Delta E$ is optimal, i.e. $\partial F / \partial \beta=\partial F / \partial s_{0}=0$.

With this model in mind we now explain several observations done during this study.

\section{Initial current}

According to the model, see Eq. S5.6, the initial current value when the drop touches the wire at a "off-wire" mode can be calculated as $I_{0}=\sigma_{T} / c_{d} R_{L}$, depending not only on the load resistance, but also on the surface charge density $\sigma_{T}$. This prediction is also confirmed by the experimental results shown in Fig. S5.6. The currents measured in Figs. S5.6a and b, using substrates with a charge density of $-0.07,-0.20$ and $-0.35 \mathrm{mC} / \mathrm{m}^{2}$, directly show that a higher $\sigma_{T}$ results in a higher $I_{0}$. With a load resistance of $47 \mathrm{k} \Omega$, milliamp level initial currents can be achieved. But the low resistance also leads to a shorter electrical relaxation time $\left(\tau_{e l}=R_{L} c_{d} A_{\max }\right)$. The peak width of the current for $R_{L}=47 \mathrm{k} \Omega$ is below millisecond, which is much narrower than for $R_{L}=6.5 \mathrm{M} \Omega$ (inset of Fig. S5.6b). $I_{0}$ and the corresponding initial power $\left(P_{0}\right)$ are shown in Fig. S5.6d for a wide range of $R_{L}$ values. Taking into account a $10 \sim 20 \mathrm{k} \Omega$ internal resistance introduced by the detection electronics $\left(R_{\text {in }}\right)$, we fit the $I_{0}\left(R_{L}\right)$ curve with $I_{0}=\sigma_{T} / c_{d}\left(R_{L}+R_{i n}\right)$ varying $\sigma_{T}$. The fitted value of $\sigma_{T}$ is consistent with the EW measurements, as discussed in Supl. Sect. II. 

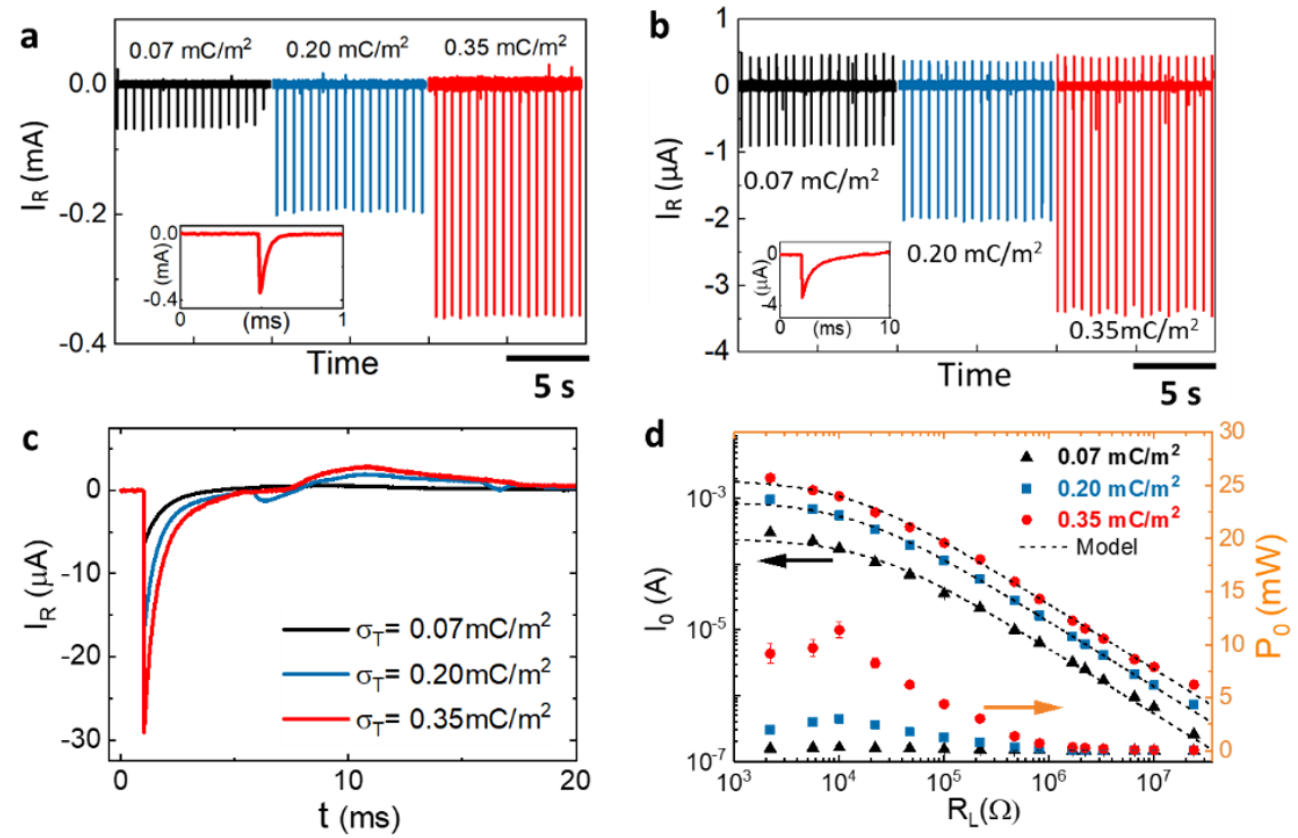

Figure S5.6 Current $I_{R}(t)$ for charge densities $\sigma_{T}$ of $-0.07,-0.20$ and $-0.35 \mathrm{mC} / \mathrm{m}^{2}$ with load resistor $\mathbf{a}, R_{L}=47 \mathrm{k} \Omega$ and $\mathbf{b}, R_{L}=6.5 \mathrm{M} \Omega$. Insets show $I_{R}(t)$ for $\sigma_{T}=-0.35 \mathrm{mC} / \mathrm{m}^{2}$. c, $I_{R}$ from a single drop impact for different $\sigma_{T}$ values $\left(R_{L}=810 \mathrm{k} \Omega\right)$. d, Initial current $I_{0}$ and power $P_{0}$ versus load resistance $R_{L}$. The dashed line shows the best fitting curve $I_{0}\left(R_{L}\right)=$ $\sigma_{T} / R_{L} c_{d}$. The substrates are the same as in Fig. S5.2.

\section{Conservation of transferred charge}

In the situation that the drop impacts on a flat surface in a "off-wire" mode, there is always a finite contacting area when the drop detaches from the wire, which cannot be neglected (see Fig. 5.1). However, after reaching the maximum spreading, the impacting drop will contract and bounce off (Fig. S5.7a). As a result, the finite area when the drop detaches from the surface could be very small (the red circle in the Fig. S5.7a). Correspondingly, almost all the charge transferred to the EDL is transferred back to the bottom electrode as the drop contracts and bounces off the surface. As shown in Fig. S5.7b, the integral over the negative part of the $I_{R}$ and the positive part are identical.

When $R_{L}$ is small, and $\tau_{e l}$ is short, all charge in the EDL near the liquid-substrate contact area can transfer back and forth between the bottom electrode and the EDL during drop spreading and contraction. However, when $R_{L}$ is large and $\tau_{e l}$ 
comparable with $\tau_{h}$, charge transfer is partially blocked by the large $R_{L}$ in the circuit. In Fig. S5.7c, is also shown that the amount of transferred charge $q$ is higher for samples with a larger surface charge density. The maximum $q$ for the samples with a surface charge of $-0.07,-0.20$ and $-0.35 \mathrm{mC} / \mathrm{m}^{2}$ are $\sim 23 \mathrm{nC}, 12 \mathrm{nC}$ and $5 \mathrm{nC}$, respectivily. Considering the maximum spreading area of a $33 \mu \mathrm{L}$ drop released from a $4.3 \mathrm{~cm}$ height is around $0.62 \mathrm{~cm}^{2}$, the transferred charge density of these three samples are approximately $0.08 \mathrm{mC} / \mathrm{m}^{2}, 0.24 \mathrm{mC} / \mathrm{m}^{2}$ and $0.37 \mathrm{mC} / \mathrm{m}^{2}$. These results are consistent with the EW results in Suppl. Sect. II.
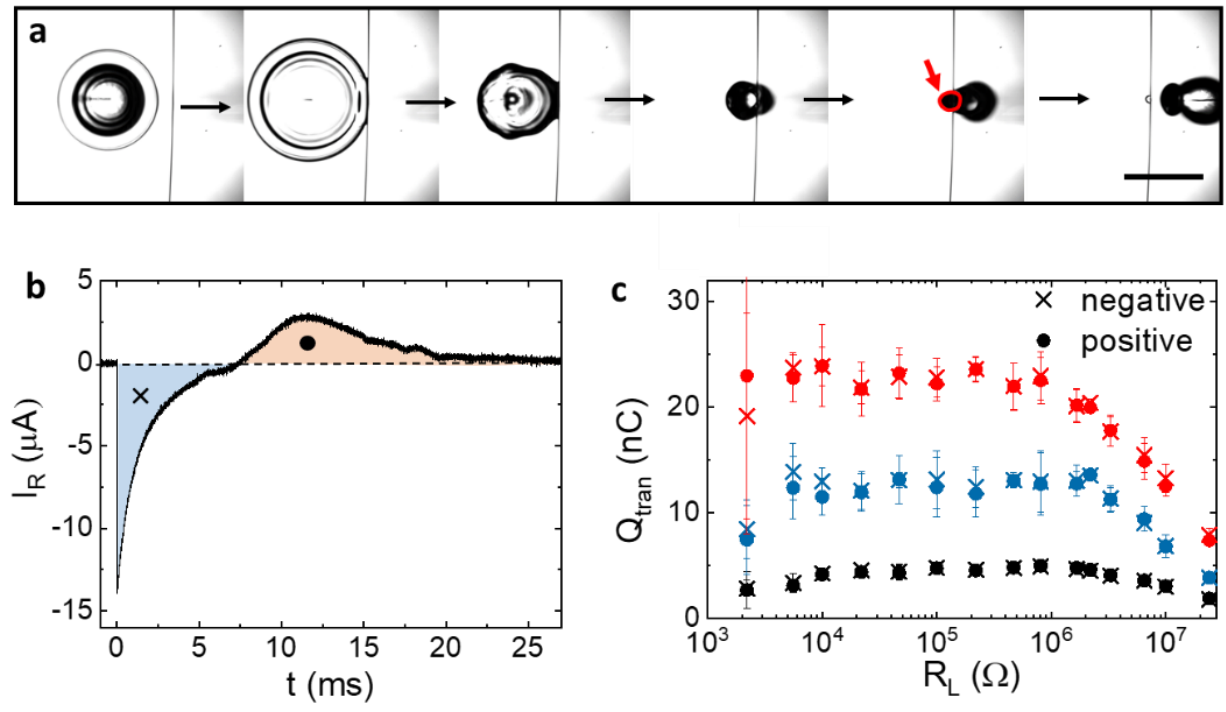

Figure S5.7 a, bottom view through a tilted transparent substrate of a drop (volume: $33 \mu \mathrm{L}$; liquid: $100 \mathrm{mM} \mathrm{NaCl}$ solution) impacting on a hydrophobic surface (Teflon AF1600). b, generated current $\left(I_{R}\right)$ from a $33 \mathrm{uL}$ drop impacting on a surface with charge density $\left(\sigma_{T}\right)$ of $0.35 \mathrm{mC} / \mathrm{m}^{2}$. c, the amount of transferred charges $\mathrm{q}$ with samples of $\sigma_{T}=-0.07$ $\mathrm{mC} / \mathrm{m}^{2}$ (black) , $-0.20 \mathrm{mC} / \mathrm{m}^{2}$ (blue) and $-0.35 \mathrm{mC} / \mathrm{m}^{2}$ (red) depending on $R_{L}$. The "x" represents current direction from the bottom to the EDL at liquid-solid interface (blue part in b), “•” represents the reverse current direction (orange part in b).

\section{Dependence of conductivity of the drop}

In a typical charge trapping electric nanogenerator (CT-ENG), the resistance of the drop can be neglected when the conductivity of the liquid is relatively high, because 
the resistance of the water drop $R_{\text {drop }} \ll R_{L}$. The current is calculated as $I_{R}(\mathrm{t})=$ $U(t) / R_{L}$, where the $U(t)$ is the potential difference across the dielectric capacitor.

However, when a low conductive liquid is used for the falling drop, $R_{\text {drop }}$ can be comparable or even larger than $R_{L}$, therefore $R_{\text {drop }}$ cannot be ignored and the current is $I_{R}(\mathrm{t})=U(t) /\left(R_{L}+R_{\text {drop }}\right)$. The initial current at the moment the drop touches the Pt wire is $I_{0}=\sigma_{T} / c_{d}\left(R_{L}+R_{\text {drop }}\right)$.

$\mathrm{NaCl}$ solutions with various concentrations were prepared to investigate the effect of the conductivity of the liquid. The linear relationship of the conductivity and the concentration of $\mathrm{NaCl}$ solutions is shown in Fig. S5.8a. The current curves for various conductivities of the drop are shown in Figs. S8b and c. For a low load resistance of $47 \mathrm{k} \Omega, I_{0}$ increases with increasing conductivity over a large range. $I_{0}$ becomes constant when the $\mathrm{NaCl}$ concentration reaches $0.1 \mathrm{M}$ and $R_{\text {drop }}$ becomes negligible compared to $R_{L}$. For a relatively high load resistance of $10 \mathrm{M} \Omega, R_{\text {drop }}$ hardly affects $I_{0}$, and only when the $\mathrm{NaCl}$ concentration is below $1 \mathrm{mM}$, the $I_{0}$ value can be reduced. In Fig. S5.8d $I_{0}$ versus $R_{L}$ is shown for various salt concentrations. By varying $R_{\text {drop }}$ we obtain the best fitting curve $I_{0}\left(R_{L}\right)=\sigma_{t} / c_{d}\left(R_{L}+R_{\text {drop }}\right)$. The values found for $R_{d r o p}$ are inversely proportional to the liquid conductivity. Low conductive drops introduce a certain resistance to the circuit, and as such $R_{\text {drop }}$ consumes energy. Consequently, the energy $\Delta E$ generated in the load resistor will be reduced by the low drop conductivity (Fig. S5.8f). 

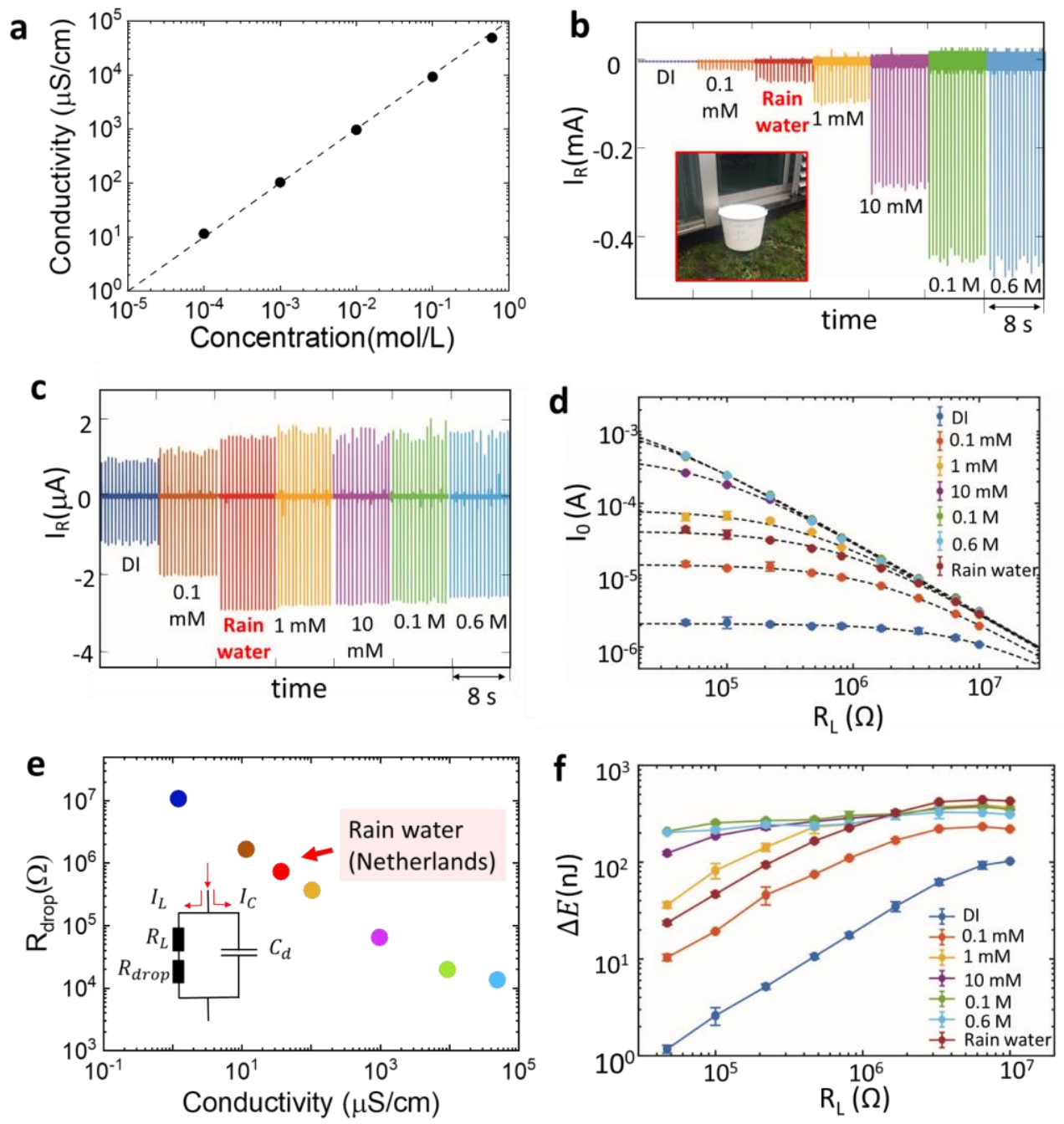

Figure S5.8 a, conductivity depending on the salt $(\mathrm{NaCl})$ concentration. Current $\left(I_{R}\right)$ generated from multiple falling drops with various salt concentration, as well as the rain water from the campus of University of Twente (Enschede, the Netherlands) with load resistance of $\mathbf{b}, 47 \mathrm{k} \Omega$ and $\mathbf{c}, 10 \mathrm{M} \Omega$. Inset of $\mathbf{b}$ shows rain water collection. $\mathbf{d}$, the characteristic current value $I_{0}$ depending on load resistance with various salt concentration of drops. e, calculated resistance induced by drops $\left(R_{\text {drop }}\right)$ depending on drop conductivity. f, generated energy ( $\Delta E$ ) depending on load resistance with various salt concentration of drops. 


\section{Calculating the harvested energy $\Delta E$}

Using Eqs. S5.4 and S5.11, and a typical area profile $A=A_{\max } f(s)$ of a drop that impacts on a hydrophobic surface (Fig. S5.9a), we calculate the energy $\Delta E=$ $E_{0} g\left(\beta, s_{0}, s_{f},\left\{\alpha_{n}\right\}\right)$, where $g$ depends on the load resistance, via $\tau_{e l}=\tau_{h} / \beta=$ $R_{L} c_{d} A_{\text {max }}$. The results have been shown in Fig. S5.9b. From this calculation we learn that for $\tau_{e l}<\tau_{h}$ most energy is harvested when the drop touches the Pt wire at maximal spreading.
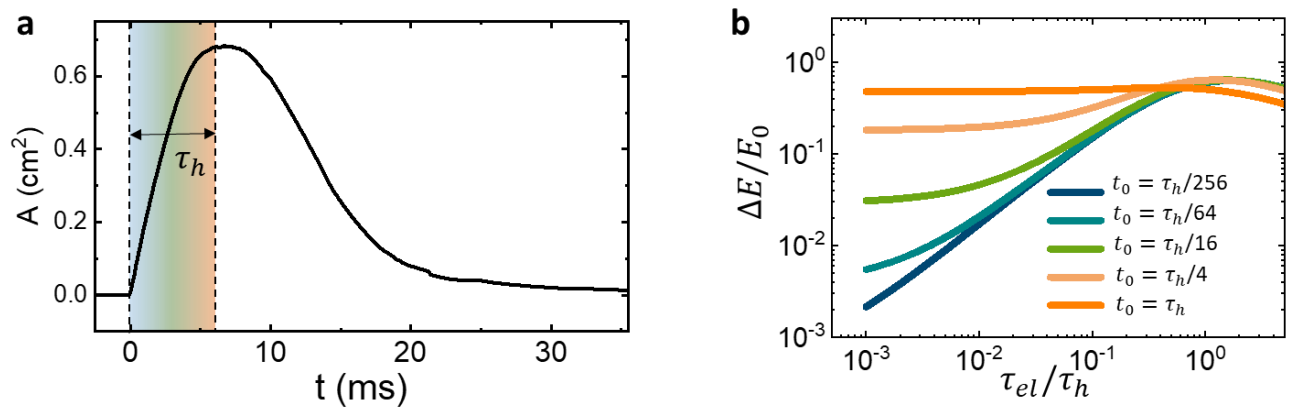

Figure S5.9 a, Typical time profile of the contacting area $A(t)$ for a water drop impacting on a hydrophobic surface. $\mathbf{b}$, Calculated $\Delta E / E_{0}$ versus $\tau_{e l} / \tau_{h}$ for various drop impact times $(0<$ $\left.t_{0} \leq \tau_{h}\right)$.

\section{References}

[1] Q. Sun, D. Wang, Y. Li, J. Zhang, S. Ye, J. Cui, L. Chen, Z. Wang, H.-J. Butt, D. Vollmer, Nature materials 2019, 18, 936.

[2] Z. H. Lin, G. Cheng, S. Lee, K. C. Pradel, Z. L. Wang, Advanced Materials 2014, 26, 4690 .

[3] A. Z. Stetten, D. S. Golovko, S. A. Weber, H.-J. Butt, Soft matter 2019.

[4] E. Seyrat, R. A. Hayes, Journal of Applied Physics 2001, 90, 1383.

[5] H. Wu, H. Li, A. Umar, Y. Wang, G. Zhou, Materials 2018, 11, 2474.

[6] A. G. Banpurkar, Y. Sawane, S. M. Wadhai, C. Murade, I. Siretanu, D. van den Ende, F. Mugele, Faraday discussions 2017, 199, 29.

[7] F. Boulogne, J. D. Warner, E. Neil Yager, 2014. 
Chapter 5

112 


\section{Chapter 6}

\section{Why and how to achieve a high charge density for a charge trapping electric nanogenerator (CT-ENG) ${ }^{*}$}

In Chapter 5, we developed a charge trapping electric nanogenerator (CT-ENG) and proposed a physical model to understand the working principle of CT-ENG for energy harvesting from water drops impacting on charged hydrophobic substrates. In this chapter, we explore in more detail the influence of some crucial parameters of this model on the performance of a CT-ENG. We also propose an improvement of the electrowetting assisted charge injection technique that enhances the trapped charge density in a CT-ENG device, resulting in a more efficient energy conversion.

\footnotetext{
* This chapter is based on the manuscript: H. Wu, D. van den Ende, S. van der Ham, G. Zhou, F. Mugele, Why and how to achieve ultrahigh surface charge density for charge trapping electrical nanogenerator (CT-ENG) (in preparation)
} 


\subsection{Introduction}

The term nanogenerator refers to an electric generator that is able to convert mechanical energy into electrical energy on a nanoscale ${ }^{[1]}$. With the increasing threat of energy crisis and global warming, nanogenerators have attracted more and more attention due to their light weight, low cost, high efficiency and promising potential for the next generation of renewable blue energy generators ${ }^{[2-5]}$. Although energy harvesting from rain drops (as we described in Chapter 5) is unlikely to provide sufficient energy to address the global energy crisis, the general principle of energy conversion based on capacitance variations due to fluid motion may still be promising for certain applications, considering the fact that $70 \%$ of the earth's surface is covered by oceans. More specific, the capacitance variation due to spreading as discussed in chapter 5, will also occur when ocean waves splash against a gigantic panel with suitable coatings and electrical circuit design, as conceptually described by Wang et al. ${ }^{[2]}$.

According to their working mechanism, nanogenerators are often divided in several types, such as triboelectric nanogenerators $(\mathrm{TENG})^{[6]}$, electret nanogenerators ${ }^{[7]}$, electret-triboelectric nanogenerators ${ }^{[8-11]}$ and piezoelectric nanogenerators ${ }^{[12-13]}$. The early TENG devices relied on surface charge generation at the contact area due to triboelectric effects ${ }^{[14-15]}$. However, nowadays, electret materials are frequently used for TENG to enhance the charge density on the triboelectric surface, therewith improving the performance of the TENG device ${ }^{[8-11]}$. Although the charge injection methods are different, such as triboelectricity ${ }^{[6,16]}$, electrowetting-assisted charge injection $(\mathrm{EWCI})^{[17]}$, or corona discharge ${ }^{[9,18]}$, once the charges are injected and trapped at the material surface, the electrical response is for all types dictated by the counter charge transfer in the electric circuit and this energy transfer enables their functionality. We collectively call them charge trapping electrical nanogenerators (CT-ENG). Experiments have shown that higher charge densities $\sigma_{T}$ always result in a higher energy yield in such CT-ENGs ${ }^{[19-22]}$. In Chapter 5, we have shown also theoretically, that the harvested energy from impacting water drops scales with $\sigma_{T}^{2}$, but how to optimize in this respect the performance of CT-ENG devices in general is still not well described. In practice, careful selection of substrate material and optimization of the surface structure allowed for surface charge densities up to hundreds of $\mu \mathrm{C} / \mathrm{m}^{2[20]}$, which is much higher than the intrinsic $\sigma_{T}$ of polymer films which is only several tens of $\mu \mathrm{C} / \mathrm{m}^{2}{ }^{[23-24]}$. In 2017, Wang et al. reported a surface charge of $1.003 \mathrm{mC} / \mathrm{m}^{2}$, in a high vacuum environment ${ }^{[21]}$. More recently, $\sigma_{T}$ values ranging from 1.02 to $1.25 \mathrm{mC} / \mathrm{m}^{2}$ have been achieved under ambient conditions ${ }^{[22 \text {, }}$ 25]. 
In this chapter, we will first exploit the model developed in chapter 5 to analyze the consequences for a general CT-ENG based on a contact line moving with constant speed rather than considering complex drop spreading. As we will see, the model will illustrate the general validity of our previous conclusion that the generated energy is proportional to $E_{0}=\sigma_{T}^{2} A_{\max } / c_{d}$ for all types of CT-ENG with a proportionality constant that is of the order one under optimal conditions. Here $A_{\max }$ is again the maximal contact area between the liquid and charged substrate, while $c_{d}$ is the capacitance per unit area of the dielectric films. Next, we will address the specific technical challenge to maximize the trapped charge density while maintaining long term stability. Based on the EWCI method developed in chapter 4, we will show that charge densities up to $1.8 \mathrm{mC} / \mathrm{m}^{2}$ can be achieved in a large area instead of a microline region by optimizing substrate material, fluid composition, and electrical charging conditions. In this manner, we obtain an overall conversion efficiency of up to $10 \%$ from initial kinetic impact energy to electrically harvested energy. Moreover, we will demonstrate a simple technique to determine the surface charge density on the substrate by measuring the induced current during the liquid motion over the substrate.

\subsection{A case study, a sliding mode CT-ENG}

To reveal the parameters that control CT-ENG, we first study a simple case in which a top electrode slides with a constant speed on a charged surface (Figure 6.1). Depending on the charge generation method used, the CT-ENG could be referred to as a triboelectric, an electret, an electret-triboelectric or an electrowetting-assisted charge injection (EWCI) nanogenerator. The moving top electrode can be any conductive material, such as water, a liquid or solid metal. In this case study, we assume that the top electrode slides smoothly over the dielectric substrate with a stable surface charge density $\sigma_{T}$. Before the top electrode reaches the dielectric substrate (left panel in Figure 6.1a), all the counter charge induced by $\sigma_{T}$ resides on the bottom electrode. Once the top electrode starts moving over the dielectric layer, counter charge is transferred from the bottom to the top electrode (Figure 6.1a). 
(a) Starting state

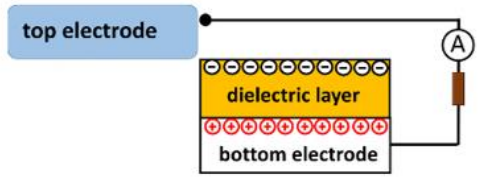

(b)

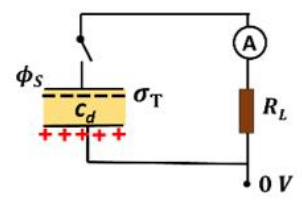

Sliding
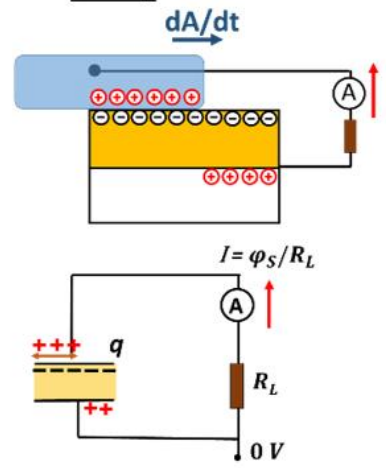

Final state
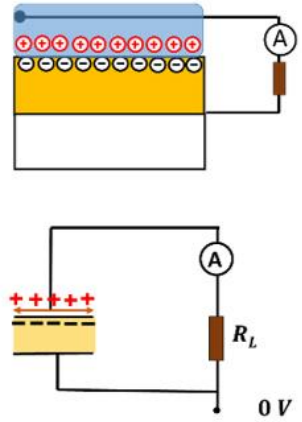

(c)

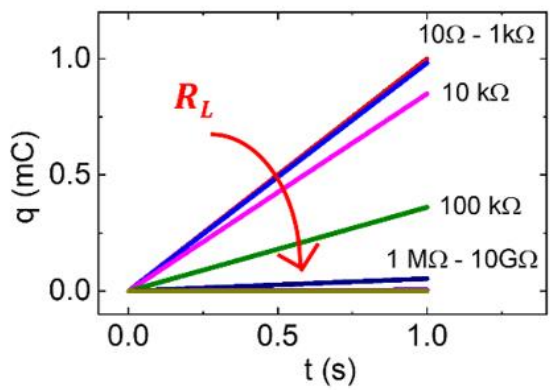

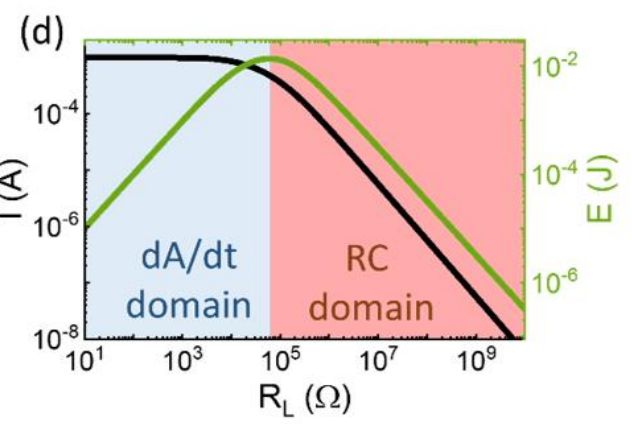

Figure 6.1. (a) Schematic of charge trapping electrical nanogenerator (CT-ENG) with the top electrode (water, liquid metal or metal, etc.) moving on dielectric surfaces with a surface charge of $\sigma_{\mathrm{T}}$. The interfacial area variation ratio is $\mathrm{dA} / \mathrm{dt}$ (b) The equivalent circuit of sliding mode CT-ENG. The bottom electrode is always grounded. $\varphi_{\mathrm{s}}$ is the surface potential of the dielectric film, here, $. \varphi_{\mathrm{s}}=U ; \mathrm{q}$ is the amount of charges transferred to the top electrode. $\mathrm{c}$ is the capacitance per area of the dielectric layer. $d$ is the thickness of the dielectric layer. $R_{L}$ is the load resistance. I is the current in the circuit. The influence of load resistance on (c) the amount of transferred charges (q) depending on time. (d) the current value and generated energy (E) depending on $R_{L}$.

The equivalent circuits are shown in Fig. 6.1b. Before the top electrode makes contact with the dielectric substrate, the initial local potential difference $U(t=0)$ between the top of the dielectric film and the bottom electrode is determined by the surface charge density $\sigma_{t}$ :

$$
U(t=0)=\sigma_{T} / c_{d}
$$

Once the top electrode starts to overlap with $\sigma_{T}$, the charge in the capacitor formed by the two electrodes is the sum of the transferred charge $q(t)$ and the initial surface 
charge at the contact area $\sigma_{T} A(t)$. The surface potential is calculated as:

$$
U(t)=\frac{q(t)+\sigma_{T} A(t)}{c_{d} A(t)}
$$

while the current $I(t)$ through the resistor $R_{L}$ is given by:

$$
I(t)=-\frac{d q}{d t}=\frac{U(t)}{R_{L}}
$$

Here we define the current from the top to the bottom electrode (ground) as positive. Combining Eqs. 6.2 and 6.3, we get:

$$
\frac{d q}{d t}=\frac{-\sigma_{T}}{R_{L} c_{d}}-\frac{q(t)}{R_{L} c_{d} A(t)}
$$

Because the top electrode slides at constant speed $v$ over the substrate, the contact area is given by $A(t)=B v t$, where $B$ is the width of the electrode. In this case we observe by inspection that the solution of Eq. S6.4, with $q(t=0)=0$, is simply given by: $q(t)=I_{0} t$ where

$$
I_{0}=\frac{-\sigma_{t} B v}{1+R_{L} c_{d} B v}
$$

Therefore, the current through the resistor is given by $I(t)=-I_{0}$ and the voltage over the resistor by $U(t)=-R_{L} I_{0}$.

To investigate the electric response in more detail, we consider the top electrode to slide over a surface of $1 \mathrm{~m}^{2}$ with a constant speed of $v=1 \mathrm{~m} / \mathrm{s}$ while the width $B=$ $1 \mathrm{~m}$ and $\sigma_{t}=1 \mathrm{mC} / \mathrm{m}^{2}$ (see also Table 1, Case 1). Figure 6.1c shows the transferred charge $q$ versus time $t$ for several values $R_{L}$ of the load resistor, as calculated with Eq. 6.5. With increasing $R_{L}$, the charge transfer in the circuit is increasingly hindered by $R_{L}$. As a result, the slope of $q$ versus $t$, i.e. the current, decreases. Figure $6.1 \mathrm{~d}$ shows that for low $R_{L}$ values, i.e. $R_{L} \ll\left(c_{d} B v\right)^{-1}$, the current is dominated by $d A / d t=B v$ and hardly depends on $R_{L}$. Due to the low resistance the $R C$ time is short and the charge transfer follows the capacity increase, caused by $d A / d t$, without delay. As a result, the total transferred charge at a short time $d t$ is $\sigma_{T} d A$, and the electric current is $I=\sigma_{t} d A / d t=\sigma_{t} B v$. For high $R_{L}$ values, the charge transfer is impeded by $R_{L}$, and only a fraction of the charge accumulated in the capacitor can be compensated. In 
this regime the charge transfer is determined by the $R C$ time of the circuit: $I=$ $\sigma_{t} /\left(R_{L} c_{d}\right)$. At the transition between these two regimes, the harvested energy:

$$
\Delta E=\int_{0}^{t_{f}} I^{2} R_{L} d t=\frac{\sigma_{t}^{2} B v t_{f}}{c_{d}} \frac{R_{L} c_{d} B v}{\left(1+R_{L} c_{d} B v\right)^{2}}
$$

reaches its maximum for $R_{L}=\left(c_{d} B v\right)^{-1}, \Delta E_{\max }=\frac{\sigma_{t}^{2} B v t_{f}}{4 c_{d}}=1 / 4 E_{0}$.

Table 6.1. Parameter settings for the calculations, shown in Figs. 6.1 and 6.2.

\begin{tabular}{ccccccc}
\hline $\begin{array}{c}\text { Calculation } \\
\text { parameters }\end{array}$ & $\begin{array}{c}d_{\text {Diel }} \\
{[\mu \mathrm{m}]}\end{array}$ & $\varepsilon$ & $\begin{array}{c}\mathrm{c}_{\mathrm{d}} \\
{[\mathrm{F} / \mathrm{m} 2]}\end{array}$ & $\begin{array}{c}\sigma_{t} \\
{\left[\mathrm{mC} / \mathrm{m}^{2}\right]}\end{array}$ & $\begin{array}{c}d A / d t \\
{\left[\mathrm{~m}^{2} / \mathrm{s}\right]}\end{array}$ & $\begin{array}{c}\mathrm{A}_{\max } \\
{\left[\mathrm{m}^{2}\right]}\end{array}$ \\
\hline Case 1 & 1 & 2 & $1.77 \times 10^{-5}$ & 1 & 1 & 1 \\
Case 2 & 1 & 2 & $1.77 \times 10^{-5}$ & $0.5-2.5$ & 1 & 1 \\
Case 3 & - & - & $10^{-5}-5 \times 10^{-5}$ & 1 & 1 & 1 \\
Case 4 & 1 & 2 & $1.77 \times 10^{-5}$ & 1 & 1 & $0.01-100$ \\
Case 5 & 1 & 2 & $1.77 \times 10^{-5}$ & 1 & $0.01-100$ & 1 \\
\hline
\end{tabular}

Note: for a dielectric layer with thickness of 1um and relative permittivity of 2, the $c_{d}$ is $1.77 \times 10^{-5}$.

As can be observed from Eq. 6.6 the harvested energy depends not only on $R_{L}$ but also on $\sigma_{t}, c_{d}$, and $d A / d t=B v$. In Fig. 6.2 we show the dependence of $\Delta E$ on these parameters for the cases listed in Table 6.1. According to the calculations, the influence of these parameters on the CT-ENG performance can be summarized as follows:

1) $\Delta E$ increases quadratic with $\sigma_{t}$ across the entire range of resistance values (Fig. 6.2a). Consequently, also the maximum harvested energy $\Delta E_{\max }$ is proportional to the square of $\sigma_{t}$ (Fig. 6.2e). This is consistent with previous investigations ${ }^{[19]}$.

2) The capacitance $c_{d}$ affects $\Delta E$ in the high $R_{L}$ regime, only, because in this regime, the current is dominated by the $R C$ response. From Eq. 6.6 we see that $\Delta E_{\max }$ is inversely proportional to $c_{d}$. 
3) The harvested energy is proportional to the area $A_{\max }=B v t_{f}$ of the capacitor in the final state (Figs. 6.2c and 6.2e).

4) The maximum harvested energy $\Delta E_{\max }$ remains unaffected by $d A / d t=B v$. Variation of $d A / d t$ only leads to a shift in the $R_{L}$ value for which $\Delta E_{\max }$ is reached (Figs. 6.2d and 6.2e).

5) The calculated $\Delta E_{\max }$ is proportional to a characteristic energy $E_{0}=\sigma_{T}^{2} A_{\max } / c_{d}$, the proportionality constant is found to be $1 / 4$ for this case study.

These results confirm that enhancing the trapped charge density is an efficient way to enhance the harvested energy efficiency of any CT-ENG device. But the capacitance is also of large influence on the CT-ENG performance, especially in the high $R_{L}$ regime.

As demonstrated by the calculations above, for a "sliding mode" CT-ENG with a constant $d A / d t$, the maximum energy $\Delta E_{\text {max }}$ is proportional to $E_{0}=\sigma_{t}^{2} A_{\max } / c_{d}$. In Chapter V we have shown that this scaling is valid for any type of CT-ENG with an arbitrary time evolution of the liquid-substrate contact area $A(t)=A_{\max } f(t)$. There we found: $\Delta E=E_{0} g\left(\tau_{h} / \tau_{e l}, t_{o} / \tau_{h}, t_{f} / \tau_{h},\left\{\alpha_{n}\right\}\right)$ where $\tau_{h}$ is the time at maximal spreading, $\tau_{e l}=R_{L} c_{d} A_{\max }$ the $R C$ time of the circuit, $t_{0}$ the time the water touches the Pt wire, $t_{f}$ the time the water detaches from the Pt wire and $\left\{\alpha_{n}\right\}$ the coefficients describing the time profile $f(t)$. The maximum energy $\Delta E_{\max }$ is given by $\Delta E_{\max }=$ $E_{0} g_{\max }$, where $g_{\max }$ is the maximal value of $g$, i.e. when the partial derivatives of $g$ are zero: $\partial g / \partial\left(\tau_{h} / \tau_{e l}\right)=0, \partial g / \partial\left(t_{0} / \tau_{h}\right)=0$, etc. 
(a)
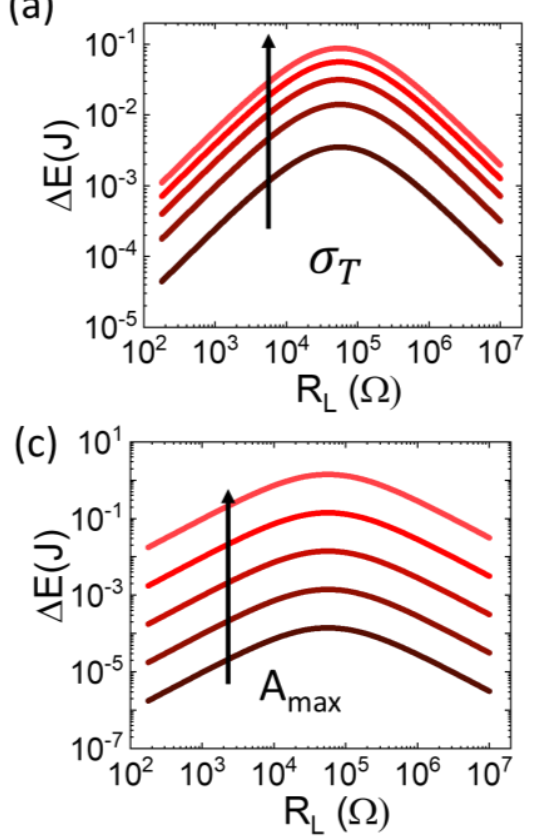

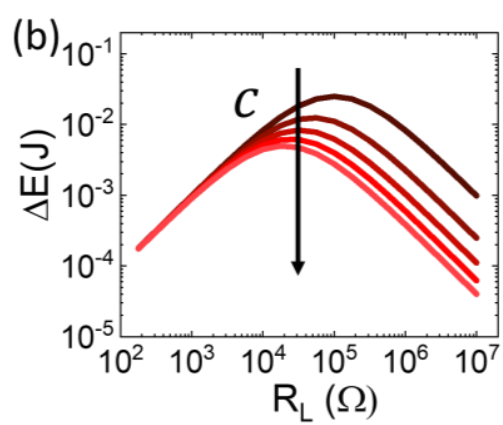

(d)

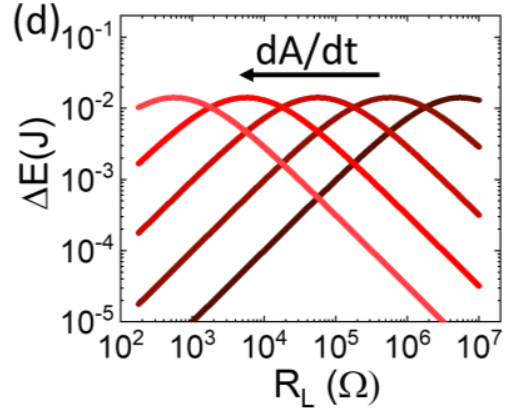

(e)

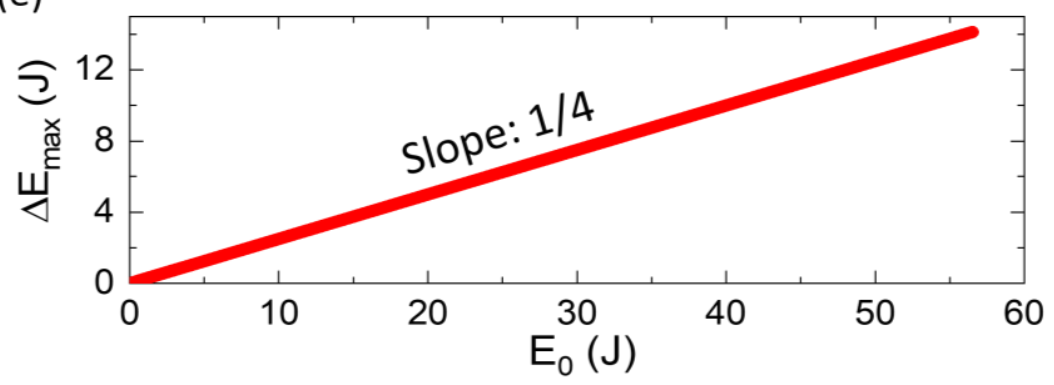

Figure 6.2 The harvested energy $(\Delta \mathrm{E})$ depending on load resistance with various (a)surface charge density $\left(\sigma_{\mathrm{T}}\right.$, Case 2 in Table 1 ) (b) capacitance per area (Case 3 in Table 1), (c) maximum area $\left(A_{\max }=B v t_{f}\right.$, Case 4 in Table 1$)$, and (d) ratio of contact area change $(d A / d t=B v$, Case 5 in Table 1$)$. (e) The maximum harvested energy $\left(\Delta \mathrm{E}_{\max }\right)$ depending on $\mathrm{E}_{0}\left(E_{0}=\sigma_{t}^{2} A_{\max } / c_{d}\right)$. 


\subsection{How to achieve a high $\sigma_{t}$ ?}

According to Chapter 4, surface charge can be deposited stably at an amorphous fluoropolymer (AFP)-electrolyte interface by electrowetting-assisted charge injection (EWCI) applying a relatively high electric field. The negative charge is firmly trapped at the AFP surface after switching off the voltage and removing the electrolyte. However, the maximum field in an AFP layer is limited by the dielectric strength of the AFP film. The strength typically ranges from $20 \mathrm{~V} / \mu \mathrm{m}$ to $150 \mathrm{~V} / \mu \mathrm{m}$, and depends highly on the quality and preparation method of the AFP film ${ }^{[26-28]}$. Consequently, the surface charge density during charging (in the electric double layer near the surface) is also restricted from -0.3 to $-2.5 \mathrm{mC} / \mathrm{m}^{2}$. To allow for higher electric fields upon charging, we introduce a layer of thermally grown $\mathrm{SiO}_{2}$ between the AFP film and $\mathrm{Si}$ substrate. This $\mathrm{SiO}_{2}$ layer has a high electric strength $(>1 \mathrm{kV} / \mu \mathrm{m}){ }^{[29]}$ and prevents break down of the whole $\mathrm{SiO}_{2}$-AFP dielectric layer (Figure 6.3a). By protecting the three phase contact line region (TPCL) with a polypropylene tape we suppress undesired effects close to the contact line and can therefore apply a high and homogeneous electric field to the $\mathrm{SiO}_{2}$-AFP layer over a square centimeter sized area . For a $\mathrm{SiO}_{2}$ layer with a thickness of $400 \mathrm{~nm}$ and coated with a $1.2 \mu \mathrm{m}$ thick Cytop film, charging voltages up to $400 \mathrm{~V}$ can be applied across the dielectric layer, resulting in a surface charge density during charging of $-4.4 \mathrm{mC} / \mathrm{m}^{2}$.

To quantify the resulting surface charge density, we measure the peak current induced by the impacting drop, as described in chapter 5 . When a drop impacts on a hydrophobic AFP surface, it spreads as a 'pancake' on the solid surface. After some spreading it touches at time $t_{0}$ the Pt wire that is placed on the AFP surface as a top electrode and a current pulse is generated. Considering Eq. 6.1, at the moment $t_{0}$ the 'pancake' touches the Pt wire, the potential at the liquid/solid interface is $U\left(t_{0}\right)=$ $\sigma_{T} / c_{d}$. By substituting $U=I R_{L}$, we calculate:

$$
\sigma_{t}=I_{0} R_{L} c_{d}
$$

Therefore, the surface charge density $\sigma_{t}$ can be rapidly determined by a measuring the initial value $I_{0}$ of the resulting current.

In Fig. 6.3 we explored the performance of this improved EWCI by comparing a pristine $\mathrm{AFP} / \mathrm{SiO}_{2}$ substrate with charged $\mathrm{AFP} / \mathrm{SiO}_{2}$ substrates Fig. 6.3 a and b sketch the charging and probing procedure. Fig. $6.3 \mathrm{c}$ shows the current response of a droplet impacting on the AFP surface depending on load resistance. The dielectric layers is 
$1 \mu \mathrm{m}$ Teflon on top of $300 \mathrm{~nm} \mathrm{SiO}_{2}$ and the substrate is charged for 15 min with a voltage of $400 \mathrm{~V}$ using DI water. As shown in inset of Fig. $6.3 \mathrm{c}$, the current peak value of $I_{0}$ is inversely proportional to the resistance, which confirmed the surface charge density calculation method shown in Eq. 6.11. The measured surface charge density shown in Fig. $6.3 \mathrm{c}$ is $0.35 \mathrm{mC} / \mathrm{m}^{2}$.

To further enhance $\sigma_{t}$, the first parameter we consider is the composition of the liquid. As reported before, negative charges preferentially adsorb at AFP surfaces and, based on $\mathrm{pH}$-dependent electrokinetic measurements, it was argued that the relevant charge carriers are hydroxide ions ${ }^{[30-32]}$. Our previous observations also indicated that AFP materials even spontaneously absorb a permanent negative surface charge upon extended contact with water ${ }^{[32]}$. Similarly, using a water solution with a high $\mathrm{pH}$ during exposure, enhances the (negative) charge density ${ }^{[33]}$. These observations indicate a strong affinity of hydroxide ions to the AFP surface. Therefore, we assume that electrolyte solutions with a high $\mathrm{pH}$ will also enhance the trapped charge density in our EWCI process. By using Cytop materials, the $\sigma_{t}$ indeed increases with the $\mathrm{pH}$ value of the electrolyte (Figs. 6.3d and 6.3e). Using an electrolyte with $\mathrm{pH} 11$, a surface charge density of $-0.54 \mathrm{mC} / \mathrm{m}^{2}$ is achieved. (Please be noticed, the $\mathrm{pH}$ dependence is shown for Cytop materials, however, we did not find this phenomenon for Teflon materials purchased from Chemours company)

(a)

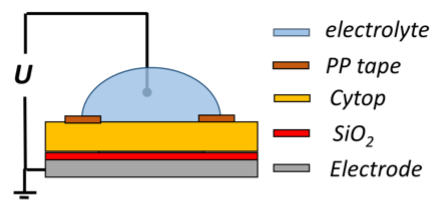

(d)

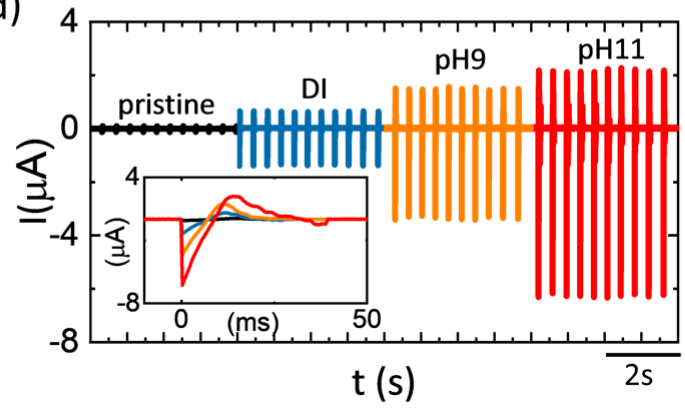

(b)

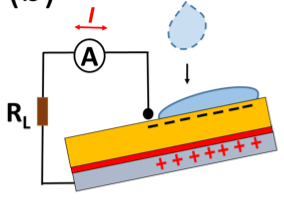

(c)

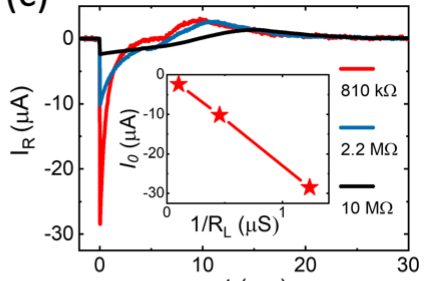

(e)

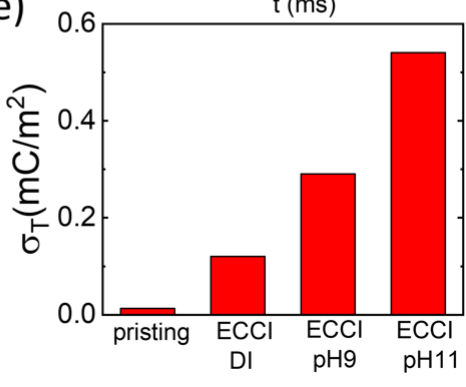

Figure 6.3. (a) schematic of optimized EWCI method and (b) surface charge density measurement method. (c) Curves of current generated by a falling droplet with different load 
resistance $\left(R_{L}\right)$. Inset shows the current peak value depending on $1 / R_{L}$. (d) Comparison of currents generated by multiple droplets with pristine AFP film (black) and the EWCI treated AFP with DI water (blue), $\mathrm{pH} 9$ (orange) and $\mathrm{pH} 11$ (red) solutions with $-400 \mathrm{~V}$ charging voltage for $15 \mathrm{~min}$. Inset shows the currents generated by a single falling drop.(e) comparison of $\sigma_{T}$ generated by EWCI process with solutions with different $\mathrm{pH}$.

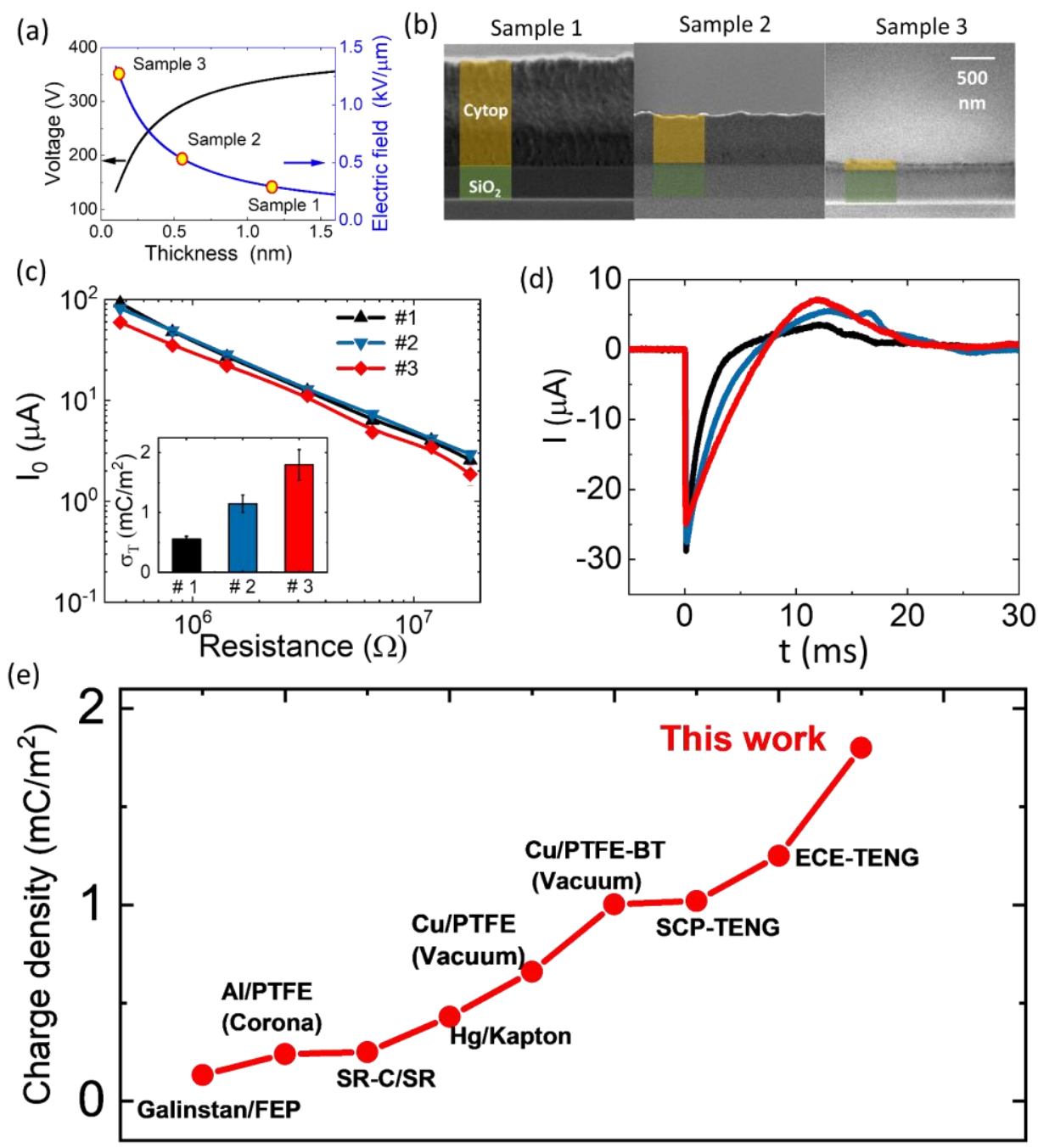

Figure 6.4. (a) The voltage drop over and electric field in the AFP layer for a total voltage of $400 \mathrm{~V}$ over both the AFP and $\mathrm{SiO}_{2}$ layer versus the thickness of the AFP layer on a sample 
with a $400 \mathrm{~nm}$ thick $\mathrm{SiO}_{2}$ layer. The thickness of AFP are $1200 \mathrm{~nm}$ for Sample 1, $500 \mathrm{~nm}$ for Sample 2 and $120 \mathrm{~nm}$ for Sample 3. (b) SEM images of the cross sections of these three samples. (c) The dependence of the current peak value $\left(\mathrm{I}_{0}\right)$ with $\mathrm{R}_{\mathrm{L}}$, and the $\sigma_{\mathrm{T}}$ (inset) using these three samples. (d) the current generated by a falling drop by using these samples. (e) comparison of the $\sigma_{\mathrm{T}}$ obtained in this work with other reports ${ }^{[25]}$.

Since the trapped charge generated by the EWCI method increases when applying stronger electric fields during charging, we can optimize the thickness of both the AFP and $\mathrm{SiO}_{2}$ layer to apply even higher electric fields to the AFP layer. Since the breakdown behavior of the dielectric layers strongly depends on the $\mathrm{SiO}_{2}$ layer, the whole dielectric film will be protected from break down by this $\mathrm{SiO}_{2}$ layer. Three samples with $400 \mathrm{~nm}$ thick $\mathrm{SiO}_{2}$ layer coated with a Cytop of varying thickness have been prepared. The thickness of Cytop layer is $1.2 \mu \mathrm{m}$ for sample $1,500 \mathrm{~nm}$ for sample 2 and $120 \mathrm{~nm}$ for sample 3. Fig. 6.4a shows both the voltage drop over the Cytop layer and the electric field inside this layer when we apply a potential difference of $400 \mathrm{~V}$ over both layers.

Figure $6.4 \mathrm{c}$ shows the measured value of $I_{0}$ as a function of $R_{L}$ generated by these three samples. By calculating $c_{d}$ with $c_{d}^{-1}=c_{1}^{-1}+c_{2}^{-1}$, where $c_{i}$ is the capacitance of each dielectric layer, and calculating $\sigma_{t}$ with Eq. 6.11, we find $\sigma_{t}=-0.56 \pm$ $0.05 \mathrm{mC} / \mathrm{m}^{2},-1.15 \pm 0.15 \mathrm{mC} / \mathrm{m}^{2}$, and $-1.80 \pm 0.25 \mathrm{mC} / \mathrm{m}^{2}$ for sample 1,2 and 3 , respectively (inset of Fig. 6.4c). The current generated from individual falling drops on these three samples is shown in Figure 6.4d. Although the initial current values $I_{0}$ for these three samples are similar, width of the current peaks increase with sample number because this width scales with the electric relaxation time $\tau_{R C}=R_{L} c_{d} A$. Compared to surface charge densities generated by other methods reported before ${ }^{[25]}$, with the optimized EWCI method much higher surface charge densities can be obtained, as shown in Figure. 6.4e.

These samples with different $\sigma_{t}$ have been used in a CT-ENG device for energy harvesting from water drops. Drops fall from a height of $5 \mathrm{~cm}$ on the $\mathrm{SiO}_{2}$-Cytop substrate that has been tilted over an angle of $30^{\circ}$. Figure 6.5a shows the process of impacting and bouncing of a drop on the surface of a CT-ENG. As shown in Figure $6.5 \mathrm{~b}$, the initial current value $I_{0}$ for all samples decreases with increasing load resistance. For the samples with identical dielectric layer composition $\left(400 \mathrm{~nm} \mathrm{SiO}_{2}\right.$ $+1.2 \mu \mathrm{m}$ Cytop), a higher $\sigma_{t}$ results in a higher $I_{0}$. For samples with different dielectric layer composition, $I_{0}$ depends also on the capacitance of the dielectric layer combination. The total transferred charge $Q_{\text {tran }}$ can be calculated by integrating the 
negative part of current versus time curve. Plotting $Q_{\text {tran }}$ as a function of $R_{L}$ for several $\sigma_{t}$ values (Figure 6.5b) shows that a higher $\sigma_{t}$ results in a higher $\mathrm{Q}_{\text {tran }}$. Moreover, $Q_{\text {tran }}$ decreases with increasing $R_{L}$ due to the increase of the electric relaxation time $\tau_{R C}$. For a CT-ENG with a relatively high capacitance (i.e. with a thin Cytop coating), $\tau_{R C}$ will increase more profoundly with increasing $\mathrm{R}_{L}$, so $\mathrm{Q}_{\text {tran }}$ decreases also more profoundly.

We calculated the harvested energy based on Eq. 6.6 and the results are shown in Fig. $6.5 \mathrm{~d}$. The $\Delta E_{\max }$ for a $33 \mu \mathrm{L}$ drop falling from $5 \mathrm{~cm}$ height on sample $3\left(\sigma_{T}=\right.$ $1.8 \mathrm{mC} / \mathrm{m}^{2}$ ) reaches $1.8 \mu \mathrm{J}$. Considering the mechanical energy of the droplet is $E_{\text {drop }}=m g h=16.5 \mu \mathrm{J}$ (where $m$ is the mass of the drop, $g$ is gravitational acceleration and $h$ is the height of the drop), the energy harvesting efficiency is $11 \%$. However, for a much lower $\sigma_{t}$ than that of sample 3, the energy harvesting efficiency of sample 2 is $12 \%$, which is comparable with and even higher than that of sample 3 . This can be explained by our previous conclusion from the calculation that $\Delta E_{\max } \propto E_{0} \propto \sigma_{T}^{2} / c_{d}$. Given the thickness of the Cytop layer of sample 2 and 3, $500 \mathrm{~nm}$ and $120 \mathrm{~nm}$, the capacitances of sample 2 and 3 are $25.8 \mu \mathrm{F} / \mathrm{m}^{2}$ and $54.5 \mu \mathrm{F} / \mathrm{m}^{2}$, respectively. Because the spreading behavior of the drops on sa111mple 2 and 3 is similar, the maximum harvested energy is determined by both the surface charge density and the capacitance of the dielectric layer.

(a)
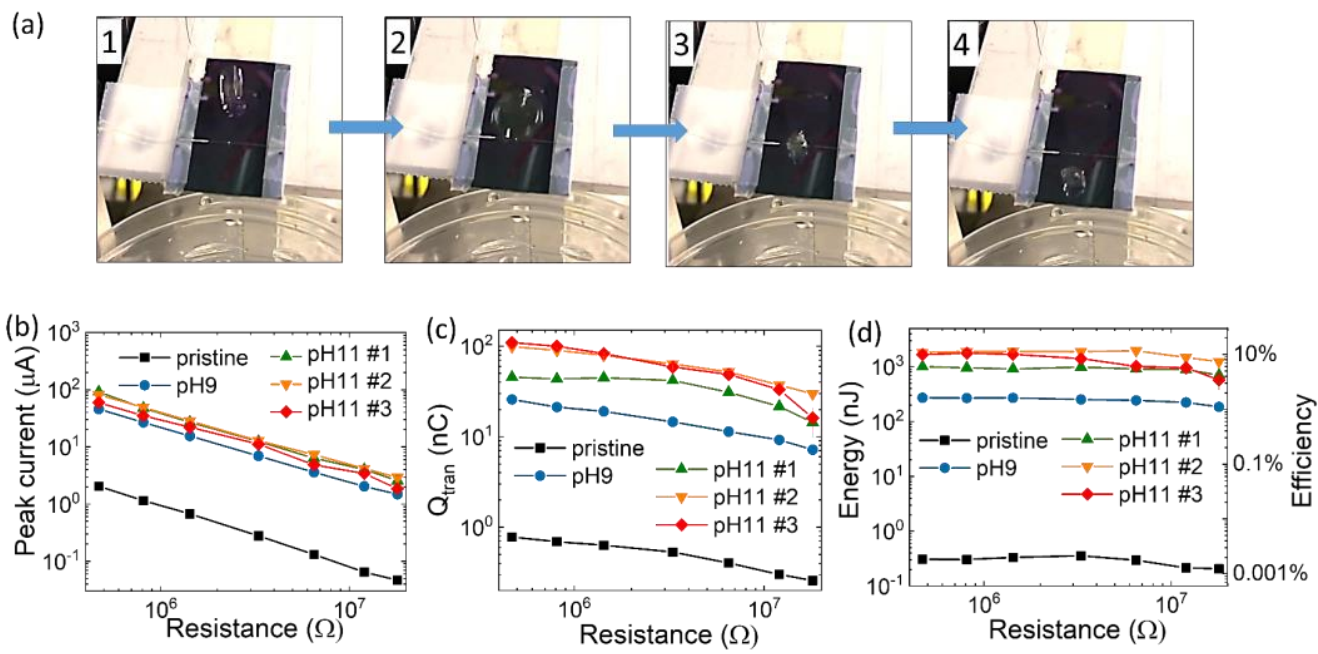

Figure 6.5. (a) The setup of CT-ENG for energy harvesting from a falling water drop. Step1: The drop falls and impacts on the charged surface. Step 2: the drop spreads and touches the 
top electrode (a Pt wire). Step 3: the drop shrinks. Step 4: the drop slides down the surface and leaves the Pt wire. The (b) current peak value, (c) total transferred charges $\left(\mathrm{Q}_{\text {tran }}\right)$ and (d) generated energy and energy harvesting efficiency of a falling drop impacting on samples before charged by EWCI (pristine), charged by $\mathrm{pH} 9$ solution for $15 \mathrm{~min}$, and charged by $\mathrm{pH}$ 11 solution with different Cytop thickness (Sample 1-3 shown in Figure 6.4)

\subsection{Conclusion}

In this chapter, the parameters that determine the performance of a charge trapping electrical nanogenerator (CT-ENG) have been investigated. Based on the numerical case study and analytical calculations, it has been found that the surface charge density $\sigma_{t}$ is an important factor, but other parameters including load resistance, wetting speed, the maximum liquid-substrate contact area and dielectric capacitance of the substrate also affect the performance of a CT-ENG. The maximum generated energy is shown to be proportional to $\sigma_{T}^{2} A_{\max } / c_{d}$. An improved EWCI process with high $\mathrm{pH}$ electrolyte and optimized dielectric layer combination is proposed to optimize the surface charge density to $\sigma_{t}=1.8 \mathrm{mC} / \mathrm{m}^{2}$ and an energy harvesting efficiency of $10 \%$ has been achieved by the proposed approach.

\section{Acknowledgements}

This work was supported by National Key R\&D Program of China (2016YFB0401501), National Natural Science Foundation of China (Grant No. 51561135014, U1601651), Program for Chang Jiang Scholars and Innovative Research Teams in Universities (No. IRT_17R40), Science and Technology Program of Guangzhou (No. 2019050001), Guangdong Provincial Key Laboratory of Optical Information Materials and Technology (No. 2017B030301007), MOE International Laboratory for Optical Information Technologies and the 111 Project.

\section{Contributions}

Hao $\mathrm{Wu}$ proposed the idea and designed the experiment. Frieder Mugele and Guofu Zhou supervised this work. Guofu Zhou and Frieder Mugele and Dirk van den Ende provided many suggestions and guidance during this work. Hao Wu performed the main experiments and did the numerical calculation. Stijn van der Ham performed the testing work related with Figure 6.3. Hao Wu analyzed the experimental data. Dirk 
van den Ende did the analytical calculations. Hao Wu wrote this chapter. Hao Wu, Frieder Mugele, Dirk van den Ende and Beybin Ilhan revised this chapter.

\section{References}

[1] Z. L. Wang, J. Song, Science 2006, 312, 242.

[2] J. Chen, J. Yang, Z. Li, X. Fan, Y. Zi, Q. Jing, H. Guo, Z. Wen, K. C. Pradel, S. Niu, ACS nano 2015, 9, 3324.

[3] U. Khan, S.-W. Kim, ACS nano 2016, 10, 6429.

[4] X. Fan, J. Chen, J. Yang, P. Bai, Z. Li, Z. L. Wang, ACS nano 2015, 9, 4236.

[5] F. Wu, C. Li, Y. Yin, R. Cao, H. Li, X. Zhang, S. Zhao, J. Wang, B. Wang, Y. Xing, Advanced Materials Technologies 2019, 4, 1800216.

[6] F.-R. Fan, Z.-Q. Tian, Z. L. Wang, Nano energy 2012, 1, 328.

[7] Y. Suzuki, IEEJ Transactions on Electrical and Electronic Engineering 2011, 6, 101.

[8] S. Wang, Y. Xie, S. Niu, L. Lin, C. Liu, Y. S. Zhou, Z. L. Wang, Advanced Materials 2014, 26, 6720.

[9] T. Zhou, L. Zhang, F. Xue, W. Tang, C. Zhang, Z. L. Wang, Nano Research 2016, 9, 1442.

[10] R. Hinchet, A. Ghaffarinejad, Y. Lu, J. Y. Hasani, S.-W. Kim, P. Basset, Nano energy 2018, 47, 401.

[11] Y. Yang, H. Zhang, X. Zhong, F. Yi, R. Yu, Y. Zhang, Z. L. Wang, ACS applied materials \& interfaces 2014, 6, 3680 .

[12] X. Wang, Nano Energy 2012, 1, 13.

[13] S. Siddiqui, H. B. Lee, D. I. Kim, L. T. Duy, A. Hanif, N. E. Lee, Advanced Energy Materials 2018, 8, 1701520.

[14] G. Zhu, C. Pan, W. Guo, C.-Y. Chen, Y. Zhou, R. Yu, Z. L. Wang, Nano letters 2012, 12, 4960.

[15] T.-C. Hou, Y. Yang, H. Zhang, J. Chen, L.-J. Chen, Z. L. Wang, Nano Energy 2013, 2, 856.

[16] Y. Xi, H. Guo, Y. Zi, X. Li, J. Wang, J. Deng, S. Li, C. Hu, X. Cao, Z. L. Wang, Advanced Energy Materials 2017, 7, 1602397.

[17] H. Wu, R. Dey, I. Siretanu, D. van den Ende, L. Shui, G. Zhou, F. Mugele, Small 2019.

[18] J. J. Shao, W. Tang, T. Jiang, X. Y. Chen, L. Xu, B. D. Chen, T. Zhou, C. R. Deng, Z. L. Wang, Nanoscale 2017, 9, 9668. 
[19] Y. Zi, S. Niu, J. Wang, Z. Wen, W. Tang, Z. L. Wang, Nature communications 2015, 6, 8376.

[20] X. He, H. Guo, X. Yue, J. Gao, Y. Xi, C. Hu, Nanoscale 2015, 7, 1896.

[21] J. Wang, C. Wu, Y. Dai, Z. Zhao, A. Wang, T. Zhang, Z. L. Wang, Nature communications 2017, 8, 88 .

[22] L. Xu, T. Z. Bu, X. D. Yang, C. Zhang, Z. L. Wang, Nano Energy 2018, 49, 625.

[23] P. Zhao, N. Soin, K. Prashanthi, J. Chen, S. Dong, E. Zhou, Z. Zhu, A. A. Narasimulu, C. D. Montemagno, L. Yu, ACS applied materials \& interfaces 2018, 10,5880 .

[24] J. Wang, Z. Wen, Y. Zi, P. Zhou, J. Lin, H. Guo, Y. Xu, Z. L. Wang, Advanced Functional Materials 2016, 26, 1070.

[25] W. Liu, Z. Wang, G. Wang, G. Liu, J. Chen, X. Pu, Y. Xi, X. Wang, H. Guo, C. Hu, Nature communications 2019, 10, 1426.

[26] E. Seyrat, R. A. Hayes, Journal of Applied Physics 2001, 90, 1383.

[27] H. Wu, H. Li, A. Umar, Y. Wang, G. Zhou, Materials 2018, 11, 2474.

[28] R. Zhou, Q. Ye, H. Li, H. Jiang, B. Tang, G. Zhou, Results in Physics 2019, 12, 1991.

[29] E. Harari, Journal of Applied Physics 1978, 49, 2478.

[30] R. Zimmermann, N. Rein, C. Werner, Physical Chemistry Chemical Physics 2009, 11,4360 .

[31] J. Lützenkirchen, T. Preočanin, N. Kallay, Physical Chemistry Chemical Physics 2008, 10, 4946.

[32] R. Zimmermann, U. Freudenberg, R. Schweiß, D. Küttner, C. Werner, Current Opinion in Colloid \& Interface Science 2010, 15, 196.

[33] A. G. Banpurkar, Y. Sawane, S. M. Wadhai, C. U. Murade, I. Siretanu, D. van den Ende, F. Mugele, Faraday Discussions 2017, 199, 29. 


\section{Summary}

This thesis mainly contains two types of electrical responsive systems. One is "electrowetting (EW)", with responsive fluoropolymer surfaces that respond to electrical stimulus. The other one is "charge trapping electric nanogenerator (CTENG)", in which the charged fluoropolymer surfaces respond to the water motion and deliver electricity.

For electrowetting applications, amorphous Fluoropolymers (AFP) are typically used as hydrophobic insulators. The AFPs are essential in electrowetting on dielectric (EWOD) systems, used either individually or by combining with an additional dielectric layer. The hydrophobicity of the AFP surfaces allow pronounced electrowetting effects to occur. The low contact angle hysteresis of AFPs make the reversible switching of the surface wettability possible. In Chapter 2, we have investigated the influence of fluoropolymers in electrowetting display devices. The performance of electrowetting display devices by using three fluoropolymers (Teflon AF, Cytop and Hyflon) have been assessed.

For some EW applications, the liquids need to be manipulated in confined regions. For example, in electrowetting display devices, the movement of the two liquids (oil and water) is controlled in many micron-sized pixels. In such a situation, the wettability contrast of the hydrophobic surfaces and the hydrophilic pixel walls is essential for the performance of the devices. In Chapter 3, we have proposed a "reconstruction" method to achieve hydrophobic/hydrophilic surfaces with ultrahigh surface wettability contrast. The performance of electrowetting display devices have been significantly enhanced by using such a method.

In EW applications, there is a long-standing issue-the charge trapping problem. It has been reported frequently in the literature that the charge trapping phenomenon will degrade the performance of the electrowetting devices. However, the microscopic investigation and a clear revelation of such a phenomenon is still missing. In Chapter 4, we have investigated the charge trapping phenomenon in a microscopic way by using three approaches of contact angle probe, electrowetting probe, and Kelvin probe force microscopy. The charge trapping is proved to be accumulated at the three phase contact line (TPCL) region and the charge density is determined by the electric field applied to the dielectric film. After revealing the phenomenon itself, we found that 


\section{Summary}

this "electrowetting-assisted charge injection (EWCI)" can be utilized as a simple and low-cost method to fabricate surface charges. The negative surface charges fabricated by such a method are very stable, even in harsh environments. Although these charges are not favored in electrowetting applications, they could be widely used in many other fields, such as nanogenerators, super capacitors and nanofluidics.

Following these findings, in Chapter 5 we proposed a novel charge trapping electric nanogenerator (CT-ENG) for generating electric power from water drops. The EWCI method has been improved by utilizing composite dielectric layers with a $\mathrm{SiO}_{2}$ layer of a higher dielectric strength. By protecting the TPCL, we have achieved centimeter sized charged areas. By using this proposed CT-ENG, electric current at mA level can be achieved (in theory, it could be infinitely high). We have investigated the working principle of CT-ENG by performing synchronized measurements of water/solid contacting area and electric current. A numerical calculation based on a physics model has predicted the electric generation process without any fitting parameters. A scaling law for energy harvesting by using CT-ENG is proposed for the optimization of the configurations.

In Chapter 6, we revealed the factors influencing the performance of CT-ENG in detail by carrying out case studies of CT-ENG in sliding mode. We have enhanced the surface charge density by optimizing the dielectric layers and the liquid $\mathrm{pH}$ during EWCI process. Surface charge density as high as $1.8 \mathrm{mC} / \mathrm{m}^{2}$ has been achieved, and the efficiency of energy harvesting from water drops reaches $10 \%$. 


\section{Samenvatting}

Dit proefschrift bespreekt hoofdzakelijk twee typen elektrisch responsieve systemen. Een systeem is "Electrowetting" (EW) met fluorpolymeer oppervlakken die reageren op elektrische stimulatie. Het andere systeem is "charge trapping electric nanogenerator (CT-ENG)", in welke geladen fluorpolymeer oppervlakken reageren op beweging van water en elektriciteit opleveren.

Voor toepassingen die gebruik maken van EW worden typisch amorfe fluorpolymeren (AFP) gebruikt als hydrofobe isolatoren. Deze AFP's zijn, eventueel in combinatie met een extra diëlectrische laag, essentieel in electrowetting op diëlectic (EWOD) systemen. De hydrofobe eigenschappen van AFP oppervlakten maken het mogelijk om het EW effect plaats te kunnen laten vinden. De lage contacthoekhysterese van de AFP's maakt het omkeerbaar wisselen in mate van oppervlaktebevochtiging mogelijk. In hoofdstuk 2 onderzoeken we de invloed van fluorpolymeren in beeldscherm systemen die gebruik maken van EW. De prestaties van deze EW beeldschermen bij gebruik van drie fluorpolymeren (Teflon AF, Cytop en Hyflon) worden beoordeeld.

Voor sommige EW applicaties worden vloeistoffen in beperkte ruimtes gemanipuleerd. Een voorbeeld hiervan zijn EW beeldschermen waar de beweging van twee fase vloeistoffen (zoals olie en water) wordt gecontroleerd in vele microscopisch kleine pixels. In zo'n dergelijke situatie is de mate van verschil in bevochtbaarheid van hydrofobe oppervlakken ten opzichte van hydrofiele wanden erg belangrijk voor de prestaties van zulke systemen. In hoofdstuk 3 stellen we een "reconstructie" methode voor om hydrofobe/hydrofiele oppervlakten met een hoog bevochtigings verschil te behalen. Er wordt aangetoond dat de prestaties van EW beeldschermen significant wordt verbeterd door gebruik van deze methode.

Een lang bekende kwestie in EW applicaties is het probleem van het vastleggen/vangen van lading. In de literatuur wordt veelvuldig besproken dat het fenomeen van het vastleggen van lading de prestaties van EW apparaten verslechterd. Een microscopisch onderzoek en een heldere uitleg van dit fenomeen ontbreekt echter nog. In hoodstuk 4 hebben we dit fenomeen van vastlegging van lading onderzocht door gebruik te maken van drie methoden: contacthoek metingen, electrowetting metingen en Kelvin-sonde kracht microscopie. Het vangen van lading is bewezen 
zich op te hopen bij de drie fase contactlijn (TPCL) regio en de ladingsdichtheid word bepaald door het elektrische veld dat wordt aangebracht op de diëlectrische oppervlakte laag. Nadat dit fenomeen werd onthult vonden we dat dit "electrowettingassisted charge injection" (EWCI) kan worden gebruikt als een simpele en goedkope methode om oppervlakte ladingen te fabriceren. De negatieve oppervlakte ladingen die door deze methode worden geproduceerd zijn erg stabiel, zelfs in een ruwe omgeving. Hoewel deze oppervlakte ladingen niet zijn gewenst in EW applicaties, kunnen ze wel in vele andere velden worden gebruikt zoals nanogeneratoren, super condensatoren en nano-fluïde toepassingen.

Volgend op deze bevindingen wordt in hoofdstuk 5 een nieuwe lading vangend elektrische nanogenerator (CT-ENG) voorgesteld voor het genereren van energy uit druppels water. De EWCI methode is verbeterd door gebruik te maken van samengestelde diëlectrische lagen met introductie van een $\mathrm{SiO}_{2}$ laag met hoge diëlectrische kracht. Lading bevattende oppervlakten op centimeter grote schaal zijn bereikt bij deze aanpak, waarbij de TPCL regio wordt beschermd. Bij gebruik van deze voorgestelde CT-ENG methode worden elektrische stromen in de orde van mA bereikt (in theorie kan dit oneindig hoog oplopen). De werkingsmethode van CT-ENG is onderzocht door gelijktijdig metingen te verrichten aan water/vaste stof contact regio's en elektrische stomen. Een numerieke berekening gebaseerd op fysische modellen hebben het proces van elektrische opwekking voorspeld zonder enige fitting parameters. Een schalingswet voor energie oogsting door gebruik van CT-ENG is voorgesteld voor de optimalisatie van de configuraties.

In hoofdstuk 6 onthullen we de factoren die invloed hebben op CT-ENG in detail in het geval van de glij-modus. We verbeteren de oppervlakte ladingsdichtheid door de diëlectrische lagen en vloeistof te optimaliseren tijdens het ECWI proces. Oppervlakte ladingsdichtheden zo hoog al $1.8 \mathrm{mC} / \mathrm{m}^{2}$ en efficiëntie van energie oogsting tot $10 \%$ uit water druppels zijn bereikt. 


\section{Acknowledgement}

Some people say that the word 'Ph.D.' means 'Permanent Head Damage'. I agree with this. In China, we also have a saying of ‘不破不立 [bu: po: bu: li:]’, which means that without destruction there can be no construction. I also agree with this. I think the combination of these two descriptions is my feeling during my Ph.D. period. Over the three and half years' Ph.D. time, especially the two and half years' time in University of Twente, I invested a lot, and I also gained a lot. I will always remember the darkness when I used to walk out of the office at midnight, and the brightness of the hope in my heart. Although life is not always smooth, I still feel I am a lucky girl, because every time when I sank into the depths of hopelessness, there would be someone appearing and shedding a light in my life. I am grateful to those people, and also thankful to fate and life.

First of all, I would like to thank my two supervisors, Prof. Frieder Mugele and Prof. Guofu Zhou. I will never forget, on $18^{\text {th }}$ April, 2017, Guofu wrote an email to Frieder and recommended me as an exchange Ph.D. student from South China Normal University to University of Twente for 6 months. It impressed me a lot that at that time, Guofu was so supportive and provided me this opportunity without any hesitation. On $22^{\text {nd }}$ April, 2017, I had a skype interview with Frieder and I got the formal invitation letter on $24^{\text {th }}$ April, 2017. Probably these are only very little tiny events for my supervisors, but it means a lot for me at that special time in my life. After that, I joined PCF group in Sep. 2017 and got the approval to become a double $\mathrm{Ph}$.D. degree candidate in early 2018. I could not get this opportunity without Guofu and Frieder's support and I am grateful for that. In the following time in the PCF group, I got many opportunities to discuss with Prof. Frieder Mugele, the famous scientist in the Electrowetting society whom I just heard of before coming to PCF. Even though, sometimes, the discussions were a bit intensive, I still appreciate it a lot for the time Frieder spent on me. I learned a lot from Frieder, and his passion for great science and his serious attitude on research will always have an influence on me. I also feel very grateful to Guofu's support over all these years. Every time when I needed Guofu's help, he was always present. 
Dirk, I don't know how to express my gratitude towards you. You are like an angel teacher during these two and half years' time. Two years ago, I didn't even know how to do a calculation with Matlab. You were so patient and taught me the basic physics calculations in person four hours a week for about half a year's time. I told you at that time that, you are the first person who taught me like this, and that is true. Thank you very much for teaching me, and also for the help you provided during those hard times when I was writing my thesis. You are a successful role model and everybody respects you. I hope I could also become someone like you someday, who always helps people when they are in need, and is respected and loved by everyone. I wish all the best for you!

I would like to thank Prof. Lingling Shui. She helped me a lot on the revision of several manuscripts, and I also learned a lot writing skills from her. I would like to express my special thanks for her encouragement and support to me for pursuing a Ph.D. degree in the University of Twente during the period I was not that confident. I also appreciate her for treating me a good meal at "Kungfu" restaurant on that very bad day.

Many thanks to Dr. Robert Hayes and Dr. Fahong Li for sharing their knowledge with me and I really enjoyed the discussions with them. I also would like to thank them for all their support and encouragement during the years when I was an engineer.

I would also like to thank Prof. Jan Eijkel for lending the equipment in his lab to me, the interesting Nanofluidics course he provided and also for the helpful discussions in my annual interview.

I would like to thank Prof. Jennifer .L. Herek and Prof. Bennie ten Haken for their support for my Ph.D defense.

I would like to thank my thesis committee members for their time and interest on my thesis.

I would like to thank all my colleagues in PCF group, everybody is so lovely and I feel so lucky and happy to meet and work with you guys. First, I would like to give my special thanks to my "Curly Diver Dear B"----Beybin Ilhan. It is hardly imaginable how I could spend these two and half years' time without you. You listened to me when I had problems, you accompanied me to work overtime at night almost every day, you helped me when I needed you and you encouraged me when I was down. You showed me how strong a girl could be. We share the same value and 
pursue similar dreams. I am very lucky to find a friend like you who understands me that much in this place so far away from my hometown. You always say that, I may forget this place gradually, forgetting some person's name first and finally forget yours. I promise I won't forget you. I hope I could go to Turkey someday and I hope I could also invite you to China in the future. I wish all the best for you, and you also deserve all the best. Sara, my "assistant paranymph", I would like to thank you a lot for all the fun you bring me, and for teaching me to solve differential equations. Thank you for being my "assistant paranymph", and also thank you for being a gentleman without being angry on us even when we "bully" you as an "assistant". Sachin, "SaQiu", "NanoQiu", "NSRQiu", "Handsome Qiu", thank you for helping me in the Nanofluidics course, and all the help you provided me these years. Qierui, Su and Jun, my Chinese colleagues, I would like to thank you for your company. I enjoy a lot the time walking outside with "Super cool Qierui" during the coffee time, thank you for treating me as your friend and inviting me to your home for delicious food many times. Thanks Su for always helping me when I needed you. I would like to thank Jun for the discussions and the encouragement. Simone, thank you for saving my teeth! Daniel, thank you very much for being so nice to me and all your help for me! I would like to thank Igor for his useful discussions. Michael, thank you for always smiling and being nice to me. Isabel, you helped me a lot during these years. Thank you for "grabbing" the Christmas gifts for me every year. Amrutha, my food soulmate, thank you for your company! Ashit, Duy thank you for all your help and company. I would like to thank my students Niels, Stijn and Daan for their support in my research. Also thanks Niels and Bass for helping me translate the Summary part into Dutch. I would like to sincerely thank Ranabir for his useful discussions and his help on my "Small" paper. I have spent a very good time in PCF with many (and some former) colleagues: Carla, Nathalie, Martin, Amani, Frank, Domonkos, Aram, Olena, Jelle, Stelian, Rudy, Arvind, Davood, Arjen, thank you all!

Many thanks to my colleagues in SCNU. Without their support, I could not achieve the results related with electrowetting displays presented in this thesis. I feel grateful for all the things happened when I was in SCNU, which make it possible for me to achieve all these today.

I'm truly grateful to all of the Chinese friends in Enschede for their support and company. I would like to express my thanks to Pei Zhang, Zhen Jiao, Jie Wang, Weiqiu Chen, Juan Wang, Chaofa Zhao, Kun Dai, Panfei Sun, Jinmeng Hao, Minsi Li, Quanliang Ye, Min Lin, Ye Lv, Zhiguo Zhang, Mengna Li, Lingyun 
Xie, Lanhui Li, Hao Shi, Yidan Yu, Yanshen Li, Luo Qin, Lin Chen, Ruosha Zeng, Jiaming Zhang, Dan Hu, Tao Tian, Jin Han, Chao Gao, Ning Han, Shuqin Cao, Keyan Chen, Fan Jia, Keyang Chen, Hongjian Yu, Yan Zhao, Qiurui Chen, Xiuqin Wang... Thank you all for making my life colorful these years! I will always remember the happy time we spent together and I wish you all a happy life and a bright future!

Last but not least, I would like to thank my family for all their love, understanding, and support. I am truly blessed to meet and marry my husband Yanjie Li. He lights up my life. I couldn't have made it today without his encouragement and support. I would also like to thank Yanjie's parents for raising such a great guy, and their support for us. I would like to express my deepest thanks to my parents. They are always supporting me and providing me the endless love. I love you, my dearest mom and dad! 亲爱的爸爸妈妈，我爱你们! 


\section{Publication list}

\section{Related to this thesis:}

\section{Peer reviewed publications}

1. H. Wu, R.A. Hayes, F. Li, A. Henzen, L. Shui, G. Zhou, Influence of fluoropolymer surface wettability on electrowetting display performance, Displays, 53 (2018) 47-53.

2. H. Wu, L. Shui, F. Li, R. Hayes, A. Henzen, F. Mugele, G. Zhou, Large-Area High-Contrast Hydrophobic/Hydrophilic Patterned Surface for Robust Electrowetting Devices, ACS Applied Nano Materials, 2 (2019) 1018-1026.

3. H. Wu, R. Dey, I. Siretanu, D. van den Ende, L. Shui, G. Zhou, F. Mugele, Electrically controlled localized charge trapping at amorphous fluoropolymerelectrolyte interfaces, Small, 16.2 (2020) 1905726.

4. H. Wu, N. Mendel, D. van den Ende, G. Zhou, F. Mugele. Electric generation from drops impacting onto charged surfaces. arXiv:2001.05019 (2020, submitted)

5. H. Wu, D. van den Ende, S. van der Ham, G. Zhou, F. Mugele, Why and how to achieve ultrahigh surface charge density for charge trapping electrical nanogenerator (CT-ENG) (in preparation)

\section{Conference contribution}

1. H. Wu, A. G. Banpurkar, I. Siretanu, F. Mugele. Charge trapping at fluoropolymer/water interfaces, Electrowetting 2018, Enschede, the Netherlands (best poster award)

2. H. Wu, A. G. Banpurkar, I. Siretanu, F. Mugele. Charge trapping in Teflon induced by electrowetting, Physics @ Veldhoven 2019, Veldhoven, the Netherlands (poster)

3. H. Wu, D. van den Ende, G.Zhou and F. Mugele, Harvesting energy from water droplet based on charged trapping at hydrophobic polymer surfaces. Droplets 2019, Durham, UK (poster)

4. H. Wu, N. Mendel, D. van den Ende, G. Zhou, F. Mugele. Energy harvesting from water droplet bouncing and sliding on charged fluoropolymer surfaces, Physics@ Veldhoven 2020, Veldhoven, the Netherlands (oral presentation) 
$\underline{\text { Patents }}$

1. G. Zhou, H. Wu, F. Li, R.A. Hayes. Electrofluid support plates and preparation method therefor, and electrofluid apparatus. US10310354B2

2. 吴昊, Frieder Mugele, 周国富. 一种表面电荷的测量方法和测量装置 (Application number: CN 201910876967.9)

3. 吴昊, Frieder Mugele, 周国富. 一种产生表面电荷的方法、装置和疏水绝 缘层及其应用 (Application number: CN 201910876996.5)

4. 周国富, 吴昊, Frieder Mugele. 一种快速测量表面束缚电荷密度的方法 (Application number: CN 201910876945.2)

5. 周国富, 吴昊, Frieder Mugele.一种制造图案化表面电荷的方法和疏水绝 缘薄膜及其应用 (Application number: CN 201910877948.8)

6. 周国富, 吴昊, Frieder Mugele. 一种发电方法和发电装置 (Application number: CN 201910876944.8)

\section{Other publications:}

\section{Peer reviewed publications}

1. H. Wu, B. Tang, R. Hayes, Y. Dou, Y. Guo, H. Jiang, G. Zhou, Coating and patterning functional materials for large area electrofluidic arrays, Materials, 9 (2016) 707.

2. H. Wu, H. Li, A. Umar, Y. Wang, G. Zhou, Bifunction-Integrated Dielectric Nanolayers of Fluoropolymers with Electrowetting Effects, Materials, 11 (2018) 2474.

3. C.B. Uzundal, O. Sahin, P. Aydogan Gokturk, H. Wu, F. Mugele, B. Ulgut, S. Suzer, X-Ray Photoelectron Spectroscopy with electrical modulation can be used to probe electrical properties of liquids and their interfaces at different stages, Langmuir, (2019).

4. R. Niu, M. Jin, J. Cao, Z. Yan, J. Gao, H. Wu, G. Zhou, L. Shui, Self-Healing Flexible Conductive Film by Repairing Defects via Flowable Liquid Metal Droplets, Micromachines, 10 (2019) 113

\section{Book chapter}

1. H. Wu, R.A. Hayes, Electrically Responsive Fluoropolymer Surfaces and Devices (Chapter 8), Responsive Polymer Surfaces: Dynamics in Surface Topography, John Wiley \& Sons, (2017). 


\section{About the author}

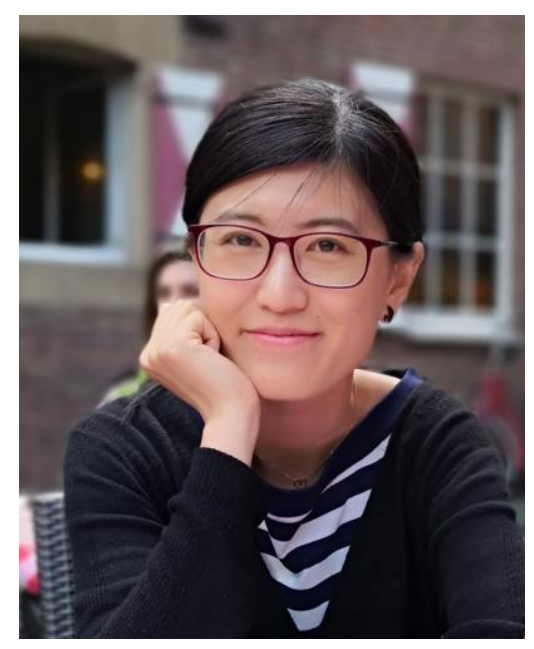

Hao Wu was born on $7^{\text {th }}$ April 1988 in Xinxiang, P. R. of China. After graduating from the High School Affiliated to Henan Normal University in 2006, Hao Wu moved to Guangzhou and entered the Department of Physics and Telecommunication Engineering at South China Normal University. In 2010, she obtained her Bachelor's degree and continued her study as a master student at the same university. After obtaining her Master's degree with a major of microelectronics and solid electronics in 2013, she started working as a R\&D engineer for promoting the industrialization of electrowetting displays. In Sep. 2016, she became a Ph.D. student in South China Normal University under the supervision of Prof. Guofu Zhou. From Sep. 15 2017, she started her research at the Physics of Complex Fluids group in the University of Twente as a double degree Ph.D. student under the supervision of Prof. Frieder Mugele and Prof. Guofu Zhou. During her Ph.D. period, she worked on interdisciplinary research of electrowetting and nanogenerators. She invented a charge fabrication method of "ElectroWetting-assisted Charge Injection (EWCI)" and a type of "Charge Trappingbased Electric Nanogenerator (CT-ENG)". The results were presented in this thesis. 